\title{
INQUIRIES IN LANGUAGE LEARNING
}

Forschungen zu Psycholinguistik und Fremdsprachendidaktik Edited by / Herausgegeben von Christiane Bongartz / Jutta Rymarczyk

Christiane M. Bongartz/Andreas Rohde (Hrsg.)

\section{Inklusion im Englischunterricht}


Ziel dieses Buches ist es, den inklusiven Englischunterricht aus einer multiperspektivischen Sicht zu beschreiben, zu seinem Kern als Prinzip der Öffnung vorzustoßen und dieses fachlich greifbarer zu machen. Die Beiträge wenden sich der Umsetzung und den für die Inklusion bedeutsamen empirischen und didaktischen Gesichtspunkten zu und sind mit den Herausforderungen der verschiedenen Kontexte befasst, in denen die VerfasserInnen wirken. Die eingenommenen Perspektiven umfassen verschiedene Förderschwerpunkte, Mehrsprachigkeit, Migrationshintergrund, schulformspezifische und inklusiv-didaktische Fragen. Es eint sie der realistische Blick auf die Praxis, die Verortung in der empirischen Forschung und evidenzbasierten Schlussfolgerungen und der Versuch, auch grundlegende Fragen zu beantworten.

Christiane M. Bongartz und Andreas Rohde sind Professoren für englische Sprachwissenschaft an der Universität zu Köln. 


\section{Inklusion im Englischunterricht}




\title{
INQUIRIES IN LANGUAGE LEARNING \\ FORSCHUNGEN ZU PSYCHOLINGUISTIK \\ UND FREMDSPRACHENDIDAKTIK
}

\author{
Edited by/Herausgegeben von \\ Christiane Bongartz und Jutta Rymarczyk
}

\section{VOLUME 17}

Zu Qualitätssicherung und Peer Review der vorliegenden Publikation

Die Qualität der in dieser Reihe erscheinenden Arbeiten wird vor der Publikation durch eine Herausgeberin der Reihe geprüft.
Notes on the quality assurance and peer review of this publication

Prior to publication, the quality of the work published in this series is reviewed by one of the editors of the series. 
Christiane M. Bongartz / Andreas Rohde (Hrsg.)

\section{Inklusion im Englischunterricht}

\section{PETER LANG}




\section{Bibliografische Information der Deutschen Nationalbibliothek}

Die Deutsche Nationalbibliothek verzeichnet diese Publikation in der Deutschen Nationalbibliografie; detaillierte bibliografische Daten sind im Internet über http://dnb.d-nb.de abrufbar.

Dieses Buch ist eine Open Access Publikation und ist auf www.oapen.org und www.peterlang.com verfügbar. Es wird auf der Basis der Creative Commons Attribution Noncommercial, No Derivatives (CC-BY-NC-ND) Lizenz verbreitet.

\section{BY-NC-ND}

ISSN 1868-386X

ISBN 978-3-631-66021-8 (Print)

E-ISBN 978-3-653-05268-8 (E-PDF)

E-ISBN 978-3-653-97188-0 (EPUB)

E-ISBN 978-3-653-97187-3 (MOBI)

DOI 10.3726/978-3-653-05268-8

(C) Peter Lang $\mathrm{GmbH}$

Internationaler Verlag der Wissenschaften

Frankfurt am Main 2015

Alle Rechte vorbehalten.

Peter Lang Edition ist ein Imprint der Peter Lang GmbH.

Peter Lang - Frankfurt am Main · Bern · Bruxelles .

New York · Oxford · Warszawa · Wien

Das Werk einschließlich aller seiner Teile ist urheberrechtlich geschützt. Jede Verwertung außerhalb der engen Grenzen des

Urheberrechtsgesetzes ist ohne Zustimmung des Verlages unzulässig und strafbar.

Diese Publikation wurde begutachtet.

www.peterlang.com 


\section{Vorwort}

Sprachenlernen/ Language Learning ist das Bindeglied, das die naturwissenschaftliche Forschung der Psycholinguistik und die anwendungsorientierte Forschung der Fremdsprachendidaktik zusammenführt. Lange Zeit wurden die Disziplinen getrennt voneinander behandelt und die Betonung lag auf den disparaten Anteilen der beiden Gebiete. Vergleichbar zur Annäherung der Begriffe „Spracherwerb“ und „Sprachenlernen“ (language acquisition und language learning) ist jedoch seit einigen Jahren eine Annäherung der Psycholinguistik und der Fremdsprachendidaktik zu beobachten. Während die Psycholinguistik den schulischen Kontext des Spracherwerbs stärker beachtet, ist aus der Fremdsprachendidaktik die empirische Forschung nicht mehr wegzudenken, die linguistische Phänomene der Interaktion im Klassenzimmer beleuchtet.

Mit der Reihe „Inquiries in Language Learning. Forschungen zu Psycholinguistik und Fremdsprachendidaktik " wollen wir dieser Entwicklung Rechnung tragen. Da die Schnittstelle der beiden Forschungsgebiete, die durch die Reihe bedient wird, naturgemäß eine hohe Interdisziplinarität aufweist, strahlt ihre Relevanz in unterschiedliche Richtungen aus: Einerseits braucht guter Fremdsprachenunterricht Lehrkräfte, deren methodisch-didaktische Entscheidungen auf detaillierter Kenntnis spracherwerbstheoretischer Aspekte beruhen. Das Schreiben von Lehrbüchern für den Fremdsprachenunterricht muss auf einer soliden empirischen Basis geschehen. Andererseits bedarf die Interpretation psycholinguistischer Daten der Vertrautheit mit Unterrichtsabläufen und den Ritualen, die Vermittlungsprozesse prägen. Das Entwerfen eines psycholinguistischen Forschungsdesigns muss unterrichtstechnische Aspekte einbeziehen, um letztlich authentische Ergebnisse abbilden zu können.

Mit der Gesamtschau unserer Arbeitsbereiche hoffen wir dem Ineinandergreifen und den Verschränkungen von psycholinguistischen Grundlagen und fachdidaktischen Weiterentwicklungen, von Theorien und Methoden sowie von Forschung und Praxis gerecht werden zu können.

Christiane Bongartz

Jutta Rymarczyk

Christiane M. Bongartz and Andreas Rohde - 978-3-653-97188-0 
Christiane M. Bongartz and Andreas Rohde - 978-3-653-97188-0

Downloaded from PubFactory at 01/11/2019 10:41:16AM

via free access 


\section{Preface}

Language Learning is a field which bridges the gap between the research conducted within Psycholinguistics and the applied research within Foreign Language Didactics. For a long time, these two fields were regarded as separate disciplines, and the emphasis lay on their differences. However, just as there has been a gradual convergence between the concepts of language acquisition and language learning, over the past few years Psycholinguistics and Foreign Language Didactics have also been moving closer together. While Psycholinguistics is taking a growing interest in the classroom context in which language learning takes place, Foreign Language Didactics have fully embraced empirical research which sheds light on the linguistic phenomena found in the interactions within the classroom.

The series Inquiries in Language Learning (Forschungen zu Psycholinguistik und Fremdsprachendidaktik) aims to reflect this development. Since the areas of intersection between these two research fields have a high level of interdisciplinarity, the contributions to this series are relevant in many different ways for educators and researchers who are concerned with language learning. On the one hand, good foreign language or second language teaching requires teachers whose methodological and pedagogical decisions are based on a sound knowledge of language acquisition theory. Furthermore, foreign language textbooks should have a solid empirical foundation. On the other hand, the interpretation of linguistic data requires familiarity with the types of classroom activities and rituals that shape the various learning processes. After all, psycholinguistic research design must attend to the technicalities of classroom teaching and learning in order to obtain authentic results.

In this series we hope to contribute to the cross-disciplinary efforts in our research fields, bringing together psycholinguistic principles and classroombased developments, thus reconciling theories and methods with research and practice.

Christiane Bongartz

Jutta Rymarczyk 
Christiane M. Bongartz and Andreas Rohde - 978-3-653-97188-0

Downloaded from PubFactory at 01/11/2019 10:41:16AM

via free access 


\section{Inhaltsverzeichnis}

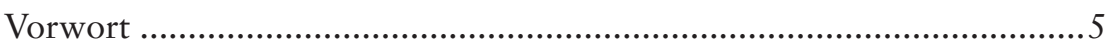

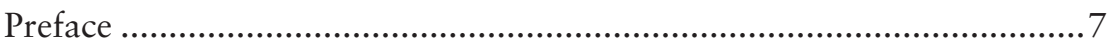

Christiane M. Bongartz \& Andreas Rohde

English for all - an introduction..............................................................11

Carolin Doert \& Günter Nold

Integrativer Englischunterricht - Forschungsfragen

zwischen Wunsch und Wirklichkeit........................................................23

Jan Springob

Die ersten Schritte sind gemacht: Inklusiver Englischunterricht an einem Gymnasium - Herausforderung und Chance............................39

Ulla Schäfer

Inklusives Lehren und Lernen im Englischunterricht ...............................57

Daniela Elsner

Inklusion von Herkunftssprachen - Mehrsprachigkeit als

Herausforderung und Chance

Jim Cummins

Inclusion and Language Learning: Pedagogical Principles

for Integrating Students from Marginalized Groups

in the Mainstream Classroom

Johanna Schnuch

Inklusion und Mehrsprachigkeit: Die Rolle von

Sprachbewusstheit im multilingualen Spracherwerb

Jens Boenisch

Zur Bedeutung von Kernvokabular im inklusiven

Englisch-Anfangsunterricht 


\section{Henning Rossa}

Lerngelegenheiten im inklusiven Englischunterricht für Schülerinnen und Schüler mit Förderbedarf im Bereich der geistigen Entwicklung

\section{Ines Steudle}

Auf dem Weg zu einer inklusiven Englischdidaktik Erkenntnisse zu Potentialen und Herausforderungen aus dem (Englisch-) Lernen von Schülerinnen und Schülern mit Förderbedarf im Bereich der geistigen Entwicklung....

Roman Bartosch \& Andreas Köpfer

Stadtnatur als Gemeinsamer Gegenstand im inklusiven Englischunterricht - Spannungsfelder und Möglichkeiten in der didaktischen Fachdiskussion

\section{Lena Kläser \& Andreas Rohde}

Fremdsprachenunterricht für gehörlose Schülerinnen

und Schüler am Beispiel des Unterrichtsfaches Englisch.

Kim Schick \& Andreas Mayer

Englischunterricht für Kinder mit Spracherwerbsstörungen.

Tatjana Leidig \& Paulina Marnett

Selbsteinschätzung im inklusiven Englischunterricht unter besonderer Berücksichtigung des Förderschwerpunktes Emotionale und soziale Entwicklung 


\section{Christiane M. Bongartz \& Andreas Rohde English for all - an introduction}

English for all - eigentlich eine ganz einleuchtende und gesellschaftlich auf der Hand liegende Forderung. Schulformübergreifende Inklusion - ein didaktisches Prinzip, das nun zur Unterrichtswirklichkeit auch im Englischunterricht gehört oder doch gehören soll, entwickelt eine Diskursdynamik, die so eigentlich ungewöhnlich ist: von Begeisterung zum Bedenkentragen, von Skepsis zu Aufbruchstimmung. Neutral ausgedrückt besteht im Umgang entweder mit dem Prinzip der Inklusion oder mit dessen Umsetzung oder mit beidem eine Verunsicherung bei den beteiligten Akteurinnen und Akteuren, die eigentlich verwunderlich ist. Das kurrikulare Antlitz des Englischunterrichts ist immer wieder wandelbar, insbesondere, was Methodik und Zielstellung des Unterrichts in den unterschiedlichen Schulformen angeht. In Nordrhein-Westfalen beispielsweise sind mit der Einführung von Englischunterricht in der Primarstufe im Jahr 2003 und der neu fokussierten Kompetenzorientierung neue Weichen gestellt worden, und im Vorfeld wie auch nach der Implementierung ergaben sich rege Diskussionen in Schulen, LehrerInnenforen und -fortbildung sowie an den Hochschulen und Universitäten. Dennoch, um die Inklusion wird anders, intensiver und auch grundsätzlicher gerungen.

Für den Englischunterricht wollen wir mit diesem Band den Versuch machen, die Abstraktion des Prinzips der Inklusion fachlich greifbarer zu machen und uns gewissermaßen wegbewegen von verengender Perzeption der Implementierung hin zum eigentlich Kern der Inklusion als einem Prinzip der Öffnung. Öffnung gutheißen können fast alle, doch wie soll sie umgesetzt werden? Um diese grundsätzliche Frage drehte sich eine von Andreas Rohde und Chris Bongartz an der Universität zu Köln im Dezember 2013 durchgeführte Konferenz, die sich aus Plenar-Vorträgen und Workshops zusammensetzte. ${ }^{1}$ Wir sind beide Sprachwissenschaftler/-in der englischen Sprache und forschen, zum Teil gemeinsam, zum Spracherwerb

1 Englisch Inklusiv! Konferenz und Workshops zu Theorie und Praxis des inklusiven Englischunterrichts, Universität zu Köln, 07.12.2013. 
des Englischen in vorschulischen und schulischen Kontexten. Aus diesem Forschungsinteresse heraus interessieren wir uns seit langem auch für $\mathrm{Li}$ teracy und Mehrsprachigkeit, und zwar zum einen in Bezug auf die psycholinguistische Seite der Sprachentwicklung und zum anderen auf deren soziokulturelle Bedeutung für Identitätsbildung, Zugehörigkeit und gesellschaftliche Teilhabe. Sicher ist es kein Zufall, dass in dem von Tony Booth und Mel Ainscow zusammengestellten inclusion index (Booth \& Ainscow 2002) insbesondere diese gesellschaftlichen Dimensionen auch von zentraler Bedeutung sind. Wenn wir also grundsätzlich auf Teilhabe hinwirken wollen, dann wird die Inklusion zum sine qua non, und mit unserem Workshop und diesem jetzt daraus hervorgegangen Buch wollen wir uns der Umsetzung und den für sie bedeutsamen empirischen und didaktischen Gesichtspunkten zuwenden.

Allerdings drängen sich für die Unterrichtspraxis im Englischunterricht wichtige Fragestellungen auf. Profitieren wirklich alle SchülerInnen vom gemeinsamen Unterricht? Gilt das für alle Schulformen? Was können wir dazu beitragen? Wie bei vielen pädagogischen Zielstellungen geht es hier einmal mehr darum, wie jede/r nach ihren/seinen Fähigkeiten optimal gefördert werden kann, eine Leitlinie, die sicher von allen LehrerInnen deutliche Zustimmung findet. Ob und unter welchen Voraussetzungen die Umsetzung gelingen kann, hängt sicher auch von Ressourcen wie guter Teamarbeit, von Förderschwerpunktliaison und Englischlehrer/-in, Klassengrößen, Material- und Medienunterstützung sowie vielfältig differenzierten interaktiven Sprachanlässen ab. Gleichzeitig wissen wir aus den ja durchaus auch vor der heutigen Debatte existierenden inklusiven Englischklassen, dass auch ein besonders differenziertes Verständnis von Zweitspracherwerbsverlauf, Lese- und Schreibentwicklung und der hier wie dort gegebenen variablen Fortschritte und Lernergebnisse wichtig sind, um eine inklusive Englischklasse so zu unterrichten, dass alle SchülerInnen angesprochen, einbezogen und gefördert werden. Gelingensbedingung in der Inklusion ist sicherlich auch, dass am Fachpersonal der SonderpädagogInnen und FachlehrerInnen nicht gespart wird. Eine professionelle Umsetzung ist nur durch ExpertInnen gewährleistet. Keiner kann alles wissen - viele ExpertInnen für Teilbereiche müssen sich zusammentun.

Mit diesem Band ist unser Ziel, eine multiperspektivische Sicht auf den inklusiven Englischunterricht einzunehmen. Diese Sicht ist notwendigerweise 
auch international, da es insbesondere im nord-amerikanischen Raum schon eine etwa fünfzig Jahre zurückreichende Tradition mit inklusiver Unterrichtsphilosophie gibt, wie sie in der magnet school-Idee zum Tragen kommen, in der inklusiv-gemischte Klassenverbände nach dem Grundsatz der Attraktivität von zum Beispiel Immersionsprogrammen in einer Fremdsprache entstehen, weil sich Eltern für diese Schulen in öffentlicher Trägerschaft entscheiden. In solchen Schulen stehen viele Ressourcen zur Verfügung, von der individuellen Zielvereinbarung für alle SchülerInnen (vgl. Nuss 2014) hin zu literacy facilitators und intensiver psychosozialer Beratung im Rahmen des Schulalltags. Empirische Befunde zum Zweitspracherwerb mehrsprachiger SchülerInnen und zur Entwicklung von und Wechselwirkung mit Lese- und Schreiberwerb wurden dort erstmals erhoben (vgl. Turnbull, Lapkin \& Hart 2001; Lazaruk 2007) und wirken für die nunmehr auch bei uns durchgeführten Erhebungsverfahren impulsgebend.

Die hier vorliegenden Beiträge sind alle unmittelbar mit den Herausforderungen der verschiedenen Kontexte befasst, in denen die VerfasserInnen wirken. Die eingenommenen Perspektiven reichen von den verschiedenen Förderschwerpunkten (Sprache, Körperliche/motorische Entwicklung, Hören, Geistige Entwicklung) über Mehrsprachigkeit und Migrationshintergrund zu schulformspezifischen und inklusiv-didaktischen Fragen. Es eint sie der realistische Blick auf die Praxis und die Verortung in der empirischen Forschung und evidenzbasierten Schlussfolgerungen. Im Einzelnen äußern sich die AutorInnen zu folgenden Themen:

Doert \& Nold betrachten zunächst die Definitionen von „integrativem“, „inklusivem“ und „gemeinsamem“ Unterricht. Überschneidungen dieser Begriffe sowie unpräzise Verwendungen verdeutlichen, dass diese im wissenschaftlichen Diskurs immer definiert werden müssen und dass diese Konzepte weiterer Elaboration bedürfen. Die Autoren konzentrieren sich auf den integrativen Englischunterricht und stellen heraus, dass es zwar noch wenige empirische Studien dazu gibt, aber dass die Förderpädagogik, Grundschuldidaktik und Fachdidaktik Ideen liefern, die nach einer kritischen Auseinandersetzung für den integrativen Englischunterricht adaptiert werden können. Doert \& Nold plädieren für den Einsatz der Muttersprache als kommunikative Lernhilfe und für den Einsatz von Task-Based-Language-Teaching zur Ermöglichung eines binnendifferenzierten Unterrichts. Dieser Ansatz wird als besonders lohnenswert für den integrativen Englischunterricht bewertet, da er 
sowohl ein gemeinsames Arbeiten als auch eine Abstimmung auf individuelle Voraussetzungen erlaubt. Die praktische Umsetzung dieses Ansatzes wird mithilfe eines Beispiels, nämlich wie Schüler ein Hörspiel aus Sherlock Holmes Short Stories kreieren können, erläutert. Eine weitere Herausforderung für Lehrkräfte, die von Doert \& Nold herausgestellt wird, ist die der Leistungsbewertung in einer integrativen Klasse, welche neue Lösungen erfordert. Team Teaching wird als unabdingbare Voraussetzung für die Erfüllung der Anforderungen von integrativem Unterricht angesehen. Aus diesen Überlegungen ergeben sich folgende Forschungsdesiderata: Es muss beobachtet werden, wie sich das fachliche, aber auch das soziale Lernen von SchülerInnen entwickelt. Ebenso muss untersucht werden, wie Lehrpläne an den Universitäten angepasst werden können, um Lehrkräfte auf den integrativen Unterricht vorzubereiten. Auch die Unterrichtspraxis selbst in integrativen Klassen erfordert eine weitere Erforschung.

Jan Springob berichtet über praktische Erfahrungen im ersten Lernjahr im Englischunterricht einer inklusiven Klasse an einem nordrheinwestfälischen Gymnasium. Gerade an Gymnasien stellt sich die Frage, wie alle SchülerInnen einen Gewinn aus dem Unterricht ziehen können. Auch hier wird darauf hingewiesen, dass die Erkenntnisse verschiedener Forschungsdisziplinen für den inklusiven Unterricht herangezogen werden müssen. Des Weiteren stellt Springob heraus, dass das Ziel des Sprachunterrichts nicht Perfektion, sondern das Erreichen kommunikativer Fertigkeiten ist. Außerdem betont er, dass jede Lerngruppe heterogen ist und dass SchülerInnen folglich individuell unterstützt werden müssen. Am Geschwister-Scholl-Gymnasium in Pulheim gibt es nun eine integrative Klasse, in der die folgenden Förderschwerpunkte vertreten sind: Lernen (3 SchülerInnen), emotionale-soziale Entwicklung (2 Schüler) und körperlich-motorische Entwicklung (1 Schüler). Im Unterricht arbeiten alle SchülerInnen manchmal gemeinsam, manchmal in Kleingruppen, aber auch getrennt. Als zentral für den Erfolg des Projektes wird die Reduzierung der Inhalte für einzelne SchülerInnen und das Vorhandensein einer klaren Struktur herausgestellt. Diese Vorgehensweise wird mit Beispielen erläutert. So wird ermöglicht, dass nach Niveaustufen unterschieden wird, aber trotzdem alle am gleichen Oberthema arbeiten können. Die SchülerInnen der Klasse empfinden den Unterricht als positiv. Um diese Eindrücke mit Daten zu hinterlegen, wird eine Vergleichsarbeit mit den 
Parallelklassen herangezogen, die aufzeigt, dass es in jeder Klasse verschiedene Leistungsniveaus gibt, und dass die inklusive Klasse unter den drei Klassen mit der besten Leistung angesiedelt ist. Auch für zieldifferent geförderte SchülerInnen wurden Möglichkeiten geschaffen, den Lernzuwachs zu überprüfen. In Zukunft werden auch weitere Kompetenzchecks notwendig sein. Außerdem werden noch angemessene Lernmaterialien entwickelt werden müssen; diese Aufgabe wird momentan noch stark vom einzelnen Lehrer getragen. Springob betont, wie unabdingbar ein gegenseitiger Austausch zwischen Wissenschaft und Unterrichtspraxis für die Weiterentwicklung von inklusivem Unterricht sei. Weitere Herausforderungen an diesen Unterricht stellen allgemein „at risk“ SchülerInnen sowie die Existenz angemessener Ressourcen. Zuletzt wird herausgestellt, dass ein „Inklusionszwang“ nicht förderlich für alle SchülerInnen wäre.

Ulla Schäfer betont in ihrem Beitrag, dass es für Lehrende wichtig ist, herauszustellen, dass inklusiver Unterricht viel mit allgemein gutem Englischunterricht gemeinsam hat. Dennoch bedarf es der richtigen personellen und materiellen Ressourcen, um den Erfolg zu ermöglichen. Anhand von Meyers (2004) Kriterien für guten Unterricht wird erläutert, wie inklusiver Unterricht gewinnbringend gestaltet werden kann. Im Sinne einer klaren Struktur kann der Lehrende Verlaufstransparenz beispielsweise mit Symbolkarten und der Verwendung von Chunks erreichen. Ebenso gehören dazu Anfangsrituale, Zieltransparenz und Endrituale. Um viel echte Lernzeit und damit Spracherwerb zu ermöglichen, sollte der Unterricht hauptsächlich in der Fremdsprache erfolgen und sinnvolle und vertraute Möglichkeiten zur Sprachverwendung bieten. Auch die Grundlagen der modernen Fremdsprachendidaktik, wie z.B. Methodenvielfalt oder Fehlertoleranz, werden als zentral für den Erfolg dargestellt. Der Unterricht sollte Freude am Sprachenlernen ermöglichen. Durch offene Aufgabenformate kann individuelle Förderung ermöglicht werden. Ebenfalls als zentral wird das Wiederholen und Üben der Lerninhalte angesehen. Außerdem sollte die Lernumgebung sinnvoll gestaltet werden. Schäfer schlägt einen Help-desk vor, der nach entsprechender Vorbereitung von allen SchülerInnen ohne Vorurteile genutzt werden kann. Wie Schäfer betont, erfordern all diese Maßnahmen neben einer erhöhten Unabhängigkeit von Lehrwerken auch die Weiterbildung von Lehrenden.

Daniela Elsner beschäftigt sich in ihrem Artikel damit, wie die sprachlichen und kulturellen Erlebnisse von Lernern mit Migrationshintergrund in 
den Englischunterricht integriert werden können. Es wird zunächst überprüft, inwiefern allgemein diskutierte Vermutungen zu den Voraussetzungen von Mehrsprachigen zum Fremdsprachenlernen zutreffen. In diesem Sinne wird erläutert, ob einsprachige und mehrsprachige Lerner die gleichen Voraussetzungen haben und ob mehrsprachige Lerner schlechter oder besser abschneiden als einsprachige. Aus der Diskussion wird ersichtlich, dass eine undifferenzierte Antwort auf diese Fragen nicht möglich ist, da verschiedene Bedingungen zu verschiedenen Formen von Mehrsprachigkeit führen. Aufgrund dieser Erkenntnis werden dann die Auswirkungen dieser Bedingungen auf das Erlernen von Fremdsprachen beleuchtet. Zu betrachten sind dort die folgenden Faktoren: der sozioökonomische Hintergrund sowie die Bildungsnähe und Unterstützung des Elternhauses, die Kompetenzen in Erst- und Zweitsprache, die persönlichen Einstellungen zur Mehrsprachigkeit und nicht zuletzt die Sprachlernumgebung. Wenn es dann um die Inklusion von Mehrsprachigen geht, ist der Fremdsprachenunterricht laut Elsner besonders geeignet, um ein Signal zur Anerkennung der Mehrsprachigkeit zu geben. Elsner gibt Anregungen, wie dies im Unterricht sowohl inhaltlich als auch methodisch umgesetzt werden kann. Auch der Reflexion von individuellen Sprachlernerfahrungen kann der Fremdsprachenunterricht Raum bieten, z.B. durch Portfolios. Dies bedeutet, dass es unabdingbar ist, dass die Lehrkräfte selbst die Inklusion von vielen Sprachen im Unterricht als wertvoll ansehen und dass im Unterricht Bezüge zu Erfahrungen und Wissen in anderen Sprachen hergestellt werden.

James Cummins' Beitrag dreht sich um die Inklusion von sozial marginalisierten SchülerInnen (d.h. solche aus Minderheitengruppen, mit Migrationshintergrund oder niedrigem sozioökonomischen Status). Es wird herausgestellt, dass die Resultate, die Schüler mit Migrationshintergrund in der Schule erzielen, oft eine Benachteiligung dieser offenlegen. Dies zeigen z.B. die PISA-Resultate, welche auch Unterschiede offenlegen, die sich mit den Einstellungen der jeweiligen Länder zum Thema Immigration verbinden lassen. Diese SchülerInnen stellen eine Risikogruppe dar, wenn Schulen es nicht schaffen, ihre Bedürfnisse zu erfüllen. Als mögliche Ursachen für die Benachteiligung dieser SchülerInnen werden drei Faktoren aufgezeigt: der Unterricht findet in einer Zweitsprache statt, der niedrige sozioökonomische Status sowie die soziale Marginalisierung, der sich diese SchülerInnen ausgesetzt sehen. Daraufhin werden Vorschläge gemacht, wie Schulen agieren 
können, um diese Faktoren und ihre Konsequenzen auszugleichen. Cummins gibt konkrete Vorschläge, die Pädagogen nutzen können, um das Bild, das sie von solchen SchülerInnen haben, zu hinterfragen. Außerdem werden Anregungen gegeben, die es Pädagogen ermöglichen, zu ergründen, inwiefern die Schule eine Beschäftigung mit Printmaterialien und identitätsbestätigende Maßnahmen unterstützt. Auch für die Planung einer Verbesserung solcher Maßnahmen wird strukturelle Unterstützung geboten.

Auch Johanna Schnuchs Beitrag beschäftigt sich mit der Inklusion von mehrsprachigen SchülerInnen und richtet dabei das Augenmerk auf die Sprachbewusstheit. Zunächst wird untersucht, was unter Sprachbewusstheit $\mathrm{zu}$ verstehen ist und ob mehrsprachige Lerner tatsächlich über eine erhöhte Sprachbewusstheit verfügen. Hierbei wird aufgezeigt, dass es begriffliche Unebenheiten gibt und verschiedene Studien zu verschiedenen Ergebnissen kommen. Daraufhin wird erläutert, wie sich Sprachbewusstheit bei mehrsprachigen Kindern mit Migrationshintergrund entwickelt und welche Auswirkungen diese auf das weitere Fremdsprachenlernen hat. Aufgrund dieser Überlegungen wird ausgeführt, wie Sprachbewusstheit im Unterricht gestärkt werden kann. Nach einer Übersicht über verschiedene Herangehensweisen, die in der Forschungsliteratur zu diesem Zwecke bereits vorgeschlagen wurden, werden konkrete Möglichkeiten für eine Umsetzung im Unterricht gegeben, sodass Sprachbewusstheit auf verschiedenen sprachlichen Ebenen gefördert werden kann.

Jens Boenisch fragt in seinem Beitrag nach der Rolle des Kernvokabulars im inklusiven Englischunterricht. Zu diesem Zwecke werden zwei Studien vorgestellt. Die erste vergleicht das Kernvokabular von Schülern mit geistiger Behinderung mit dem von Schülern, die eine Regelschule besuchen. Die zweite Studie vergleicht Schüler, die in den USA Englisch als Erstsprache sprechen mit solchen, die die Sprache als Zweitsprache lernen. So können Differenzen und Konvergenzen zwischen diesen Gruppen aufgezeigt werden. Die deutsche Studie zeigt auffallende Ähnlichkeiten im Alltagssprachgebrauch von Schülern mit und ohne Förderbedarf. Auch die prozentuale Verteilung der Wortarten ist sehr ähnlich, beispielsweise sind Pronomina in beiden Gruppen mit der höchsten Frequenz anzutreffen. Ein Lehren dieses frequenten Vokabulars im frühen Unterricht sollte demnach zu einer höheren Kommunikationsfähigkeit führen. Auch in der amerikanischen Studie deckt sich der Verlauf der Frequenz des Kernvokabulars der Muttersprachler 
und der ESL-Schüler. Solche Studienergebnisse haben zur Entwicklung von Sprachfördermaterialien aus dem Bereich der unterstützten Kommunikation geführt, die auf dem Kernvokabular basieren und geistig/körperlich beeinträchtigten Kindern Kommunikation möglich machen.

Der Artikel stellt elektronische sowie nicht-elektronische Kommunikationshilfen vor. Diese basieren auf Symbolen und ermöglichen es den Kindern ob mit oder ohne Förderbedarf im Bereich Geistige Entwicklung -, sich rasch und flexibel auszudrücken. Zum Abschluss gibt Boenisch Beispiele, die illustrieren, wie ein Fokus auf das Kernvokabular im frühen Englischunterricht einen lebendigen und flexiblen Umgang mit der Sprache ermöglicht, bei dem auch die Grammatik mitgelernt wird und der inklusiv ist.

Henning Rossa beschäftigt sich mit der Frage, wie Lerngelegenheiten für SchülerInnen mit dem Förderschwerpunkt Geistige Entwicklung geschaffen werden können. Zunächst betont der Autor, wie viel Skepsis und Zweifel das Konzept der Inklusion in Deutschland noch hervorruft und dass sich dies gerade auch bei Kindern mit Förderbedarf in der geistigen Entwicklung bemerkbar macht. Obwohl der fremdsprachliche Unterricht eigentlich gut mit Heterogenität umgehen können müsste, besteht hier noch erheblicher Handlungsbedarf. Rossas These ist, dass der Fremdsprachenunterricht lernen muss, eine Vielfalt von Bedürfnissen und Voraussetzungen anzuerkennen und diese bei der Unterrichtsgestaltung miteinzubeziehen. Obwohl das Fremdsprachenlernen nicht die höchste Priorität für geistig Behinderte hat, sollte dies, wie Rossa betont, nicht zu einem kategorischen Ausschluss der Schüler von diesem führen - nicht zuletzt, weil das Fremdsprachenlernen nebenher noch andere kognitive Prozesse trainiert. Es wird darauf verwiesen, wie wichtig die Unterteilung in kleine Schritte für diese Schüler ist, aber dass auch die Richtlinien des modernen Fremdsprachenunterrichts mit kommunikativer Ausrichtung zentral für einen solchen Unterricht sind. Dies ermöglicht es, den Unterricht an die Fähigkeiten und die Lebensinhalte der Schüler anzupassen. Die Herausforderung liegt laut Rossa darin, dass Lernende und Lehrende sich gemeinsam mit dem Fremdsprachenlernen beschäftigen.

Ines Steudle erforscht, welche Möglichkeiten und Herausforderungen der Englischunterricht von SchülerInnen mit dem Förderschwerpunkt geistige Entwicklung birgt. Genau wie Rossa betont sie, dass diese SchülerInnen nicht von der Inklusion ausgeschlossen werden dürfen. Inklusiver Unterricht muss differenziert und individualisiert erfolgen. Die Autorin stellt heraus, dass die 
allgemeine Englischdidaktik und der Englischunterricht im Förderschwerpunkt Geistige Entwicklung ein Ziel teilen: Sie wollen es Lernern ermöglichen, in der Fremdsprache zu handeln. Hierzu muss sich der Unterricht sowohl methodologisch als auch inhaltlich am Lerner orientieren, wie Steudle erläutert. Vielfalt muss wertgeschätzt werden und Lernen muss in differenzierter Form erfolgen. SchülerInnen müssen individuell mit Förderplänen unterstützt werden. Als besondere Herausforderungen für die Entwicklung eines inklusiven Unterrichts für diese Lerner bewertet die Autorin die gesellschaftlichen Einstellungen zur Inklusion, den Mangel an Unterrichtsmaterialien, die Differenzen zwischen Lehrplänen und Lebensbedeutsamkeit sowie das Thema der Leistungsbewertung. Um Inklusion zu ermöglichen, müssen Schulen die Lehrenden unterstützen und es muss schon in der Lehrerausbildung eine Präparation erfolgen.

Bartosch \& Köpfer behandeln in ihrem Beitrag Probleme und Lösungsansätze, die für den inklusiven Englischunterricht bestehen. Auch sie konzentrieren sich hierbei auf die an sich schon heterogene Gruppe der SchülerInnen mit Förderbedarf im Bereich der geistigen Entwicklung. Wie die Autoren herausstellen, ist ,Vielfalt' für didaktische Ansätze sowohl im Sinne der Methodik als auch als inhaltliches Thema von größter Relevanz. Am Modell von Feusers $(1995,1998)$ Gemeinsamen Gegenstand ausgerichtet, erarbeiten die Autoren ein beispielhaftes Aufgabendesign zum Thema ,Stadtnatur', mit dem die individuellen Bedürfnisse der Lerner berücksichtigt werden können. Gleichzeitig ist dieses handlungsorientiert und fördert die Kooperation zwischen den SchülerInnen.

Kläser \& Rohde richten ihr Augenmerk auf den Englischunterricht für gehörlose SchülerInnen, welcher sich einer Anzahl komplexer Herausforderungen stellen muss, aber dennoch eine hohe Lebensrelevanz für diese SchülerInnen hat. Nach einer Begriffsdefinition und der Charakterisierung von Gebärdensprachen als natürliche Sprachen wird auf den Erstspracherwerb gehörloser Kinder eingegangen. In diesem Zusammenhang stellen die Autoren heraus, dass es von essentieller Bedeutung ist, sicherzustellen, dass ein altersgemäßer Erwerb der Erstsprache für alle gehörlosen Kinder erfolgt. Hierzu werden die folgenden Ansätze diskutiert und evaluiert: die sukzessive Methode, Total Communication, das schwedische Modell sowie das bilinguale Konzept / die Parallele Zweisprachigkeit, wobei die beiden letzteren positiv bewertet werden. Im Anschluss daran wird erörtert, wie ein 
Englischunterricht für Gehörlose erfolgen kann. Wie die Autoren aufzeigen, beginnt die Aufgabe bereits mit der Entscheidung, welche Sprache als Unterrichts- und Zielsprache verwendet werden sollte. Sollte dies die englische Schrift- oder Lautsprache sein oder eine weitere Gebärdensprache? In diesem Fall müsste dann auch eine Entscheidung hinsichtlich American Sign Language (ASL) und British Sign Language (BSL) getroffen werden. Nach einem Ausschluss der englischen Lautsprache bieten sich immer noch verschiedene sequentielle Möglichkeiten, die anderen Fremdsprachen zu unterrichten, welche von den Autoren vorgestellt und evaluiert werden. Daraufhin werden methodologische Überlegungen zum Unterricht selbst geboten.

Schick \& Mayer erörtern, wie Englischunterricht für Kinder mit Spezifischer Spracherwerbsstörung gestaltet werden kann. Nach einer Charakterisierung dieser Lernergruppe und der daraus resultierenden Herausforderungen (Sprache ist das Haupt-Unterrichtsmedium) werden Anregungen gegeben, wie Lehrkräfte diese SchülerInnen unterstützen können. Hierbei wird insbesondere die Sprachheilpädagogik als wertvolle Quelle für Techniken aufgezeigt. Diese Anregungen werden in einer beispielhaften Englischstunde zum Thema Clothes für den inklusiven Unterricht in der Grundschule konkretisiert. Hierbei sind schon in der modernen Fremdsprachendidaktik verankerte Prinzipien für den Unterricht des Förderschwerpunktes Sprache zuträglich, allerdings werden diese noch mit der Möglichkeit zur Anpassung aus sprachheilpädagogischer Sicht ergänzt. Die diesem Unterrichtsplan zugrundeliegenden Konzepte werden detailliert erläutert, und dem Leser wird damit verdeutlicht, welche Überlegungen zur Planung einer solchen Unterrichtsstunde einfließen.

Leidig \& Marnett erarbeiten Möglichkeiten zur Selbsteinschätzung und Förderung der Sprachlernbewusstheit und Metakognition, welche insbesondere für die Stärkung des Vertrauens in die eigene Leistungsfähigkeit bedeutend sind. Der Fokus liegt hierbei auf SchülerInnen mit dem Förderschwerpunkt Emotionale und soziale Entwicklung in der inklusiven Grundschule. In kleinen Schritten wird es den Kindern möglich, ihre gelernten fremdsprachlichen Fähigkeiten zu reflektieren. Leidig \& Marnett bieten praktische Anregungen für Fragen bezüglich der Lernstrategien und -produkte sowie der Planung des zukünftigen Lernprozesses, die in Reflexionsgesprächen mit den SchülerInnen verwendet werden können. Ebenso werden Selbsteinschätzungsbögen mit Lerntipps sowie Selbsteinschätzungbögen, die sich auf 
die Inhalte des Gelernten konzentrieren, vorgestellt und deren Verwendung im Unterricht erläutert.

Die Erstellung dieses frühen Überblicks zur schulformübergreifenden Inklusion im Englischunterricht war uns ein großes wissenschaftliches Anliegen, und wir hoffen sehr, mit diesem Band auch für die Heranführung junger Lehrerinnen und Lehrer an den inklusiven Unterricht einen motivierenden Einblick in die heutigen Forschungsgegenstände, didaktischen Anliegen und weitere soziokulturelle Gesichtspunkte gegeben zu haben. Möglich wurde der Band nur durch das große Engagement vieler Beteiligter, vor allem in Workshop-Planung und -Durchführung, und wir bedanken uns sehr herzlich bei allen, die organisatorisch und inhaltlich zum Gelingen beigetragen haben und den Grundstein für diese Veröffentlichung gelegt haben, stellvertretend insbesondere bei Antje Pillunat und Kim Schick, für die gesamte Koordination. Dank gilt auch dem gesamten Editionsteam der Lehrstühle Rohde und Bongartz, besonders Julia Caratiola, für die gründliche und überlegte Redaktionsassistenz, und natürlich allen Autorinnen und Autoren. Schließlich gilt unser Dank dem Zentrum für LehrerInnenbildung der Universität zu Köln, dessen konsequente Hinwendung zu Innovation und Forschungsorientierung in der LehrerInnenausbildung an der Universität unter anderem in der finanziellen Förderung des initialen Workshops deutlich wurde.

Sicher stehen wir mit unserer Arbeit erst am Anfang. Die hier vorliegenden Kapitel belegen jedoch, dass unsere Diskussionen fruchtbar und evidenzbasiert geführt werden. Besonders optimistisch stimmt uns die Tatsache, dass hier sozusagen eine natürliche Triangulation von Förderpädagogik, Fremdsprachendidaktik und sprachwissenschaftlicher Forschung erreicht werden kann. Diese, vor allem in der LehrerInnenausbildung oft (vergeblich) gewünschte Verschränkung ist ein Zugewinn.

Köln, im Juli 2015

\section{Literaturverzeichnis}

Booth, Tony \& Ainscow, Mel (2002), Index for Inclusion: Developing Learning and Participation in Schools. Center for Studies on Inclusive Education [Online: http://www.eenet.org.uk/resources/docs/Index\%20 English.pdf 28.06.2015]. 
Feuser, Georg (1995), Behinderte Kinder und Jugendliche - zwischen Integration und Aussonderung. Darmstadt: Wissenschaftliche Buchgesellschaft.

Feuser, Georg (1998), Aspekte einer Didaktik unter Berücksichtigung tätigkeitstheoretischer und entwicklungspsychologischer Erkenntnisse. In: Eberwein, Hans (Hrsg.) (1998), Behinderte und Nichtbehinderte lernen gemeinsam. Handbuch der Integrationspädagogik. Weinheim: Beltz, 170-179.

Lazaruk, Wally (2007), Linguistic, academic, and cognitive benefits of French immersion. The Canadian Modern Language Review 63: 5, 605-628.

Meyer, Hilbert (2004), Was ist guter Unterricht? Berlin: Cornelsen Scriptor.

Nuss, Bernd (2014), Language Immersion at Waddell Language Academy, Vortrag an der Universität zu Köln, 25.11.2014.

Turnbull, Miles; Lapkin, Sharon \& Hart, Doug (2001), Grade 3 immersion students' performance in literacy and mathematics: Province-wide results from Ontario (1998-99). The Canadian Modern Language Review 58: 1, 9-26. 


\section{Carolin Doert \& Günter Nold \\ Integrativer Englischunterricht - Forschungsfragen zwischen Wunsch und Wirklichkeit}

The academic discourse on inclusion and integration in schools is affected by the fuzziness of these terms. This paper provides clarification of the terminology which can serve as an informed standard in the developing field of inclusive instruction in English as a Foreign Language. The increased heterogeneity of learner groups goes hand in hand with a methodological reorientation, e.g. a stronger role of L1 instruction as a communicative learning tool. Another step might be the systematic differentiation of lessons on the basis of Task-Based-Language-Teaching. The authors provide pertinent desiderata for future research on integrated English teaching.

\section{Begriffsdefinition - ein Versuch: Was ist integrativer oder inklusiver Unterricht?}

Die Adjektive „integrativ“ und „inklusiv“ werden im deutschsprachigen Kontext in der Diskussion über die Aufnahme von Kindern mit Beeinträchtigungen in Regelschulen verwendet. Begriffliche Unschärfen sind dabei ein Merkmal dieser Diskussion. So werden die Adjektive teils mit identischer Bedeutung gebraucht, teils aber auch mit begrifflicher Differenzierung. Darüber hinaus verstärken „Übersetzungsfehler“ aus internationalen Dokumenten wie der Salamanca-Erklärung von 1994 oder der UN-Behindertenrechtskonvention (vgl. Hinz 2012: 36; Wocken 2011: 59) die begriffliche Unklarheit. Es ist daher unumgänglich, die Begriffe genauer zu erläutern und im Hinblick auf ihre Nutzung kritisch zu reflektieren.

In der aktuellen Diskussion bezeichnet das Wort „integrativ“ auf der einen Seite ein leicht veraltetes und ,weniger angesagtes’ Konzept. Auf der anderen Seite zeichnet sich dieser Begriff durch eine relativ klar einzugrenzende Bedeutung aus. Integration ${ }^{1}$ bezieht sich danach im schulischen Kontext auf

1 Der Begriff „Integration“ wird ferner im Zusammenhang des Migrationshintergrundes verwendet. Auf diese Verwendung des Begriffes wird hier inhaltlich 
die Teilhabemöglichkeiten von Kindern und Jugendlichen mit Behinderung, das heißt auf den gemeinsamen Unterricht von beeinträchtigten und nichtbeeinträchtigten Kindern (vgl. Wocken 2011: 7). Hinz (2012: 41) beschreibt diesen Unterricht in Anlehnung an Feuser als Lernen auf unterschiedlichen Niveaus am gemeinsamen Gegenstand. Hier ist auf den ersten Blick kein inhaltlicher und theoretischer Unterschied zu Inklusion festzustellen. Wenn der Begriff „Integration“ in dieser Weise verstanden wird und es in der Inklusionsdebatte ausschließlich um den Aspekt der Beeinträchtigung geht, scheint das Begriffsfeld „Integration“ durchaus angemessen zu sein (vgl.: Hinz 2012: 49-50; Wocken 2011: 66). Gegen den Begriff der Integration spricht, dass er eine Trennung in zwei Gruppen impliziert, wobei die eine Gruppe in eine andere integriert werden soll. So besteht die Gefahr, dass Schülerinnen und Schülern mit Beeinträchtigung eine Sonderstellung zugewiesen wird. Wenn der Begriff „Integration“ in dieser Weise gedeutet wird, ist er entsprechend der neueren Diskussion nicht mehr zu rechtfertigen (vgl.: Wocken 2011: 85).

Inklusion geht im engeren Sinne sowohl im deutschsprachigen als auch im internationalen Diskurs deutlich über den Aspekt der Behinderung hinaus, insofern der Begriff mehrere Aspekte von Heterogenität wie

[...]Geschlechterrollen, ethnische Herkunft, Nationalitäten, Erstsprachen, Rassen (etwa in den USA), soziale Milieus, Religion, und weltanschauliche Orientierungen, körperliche Bedingung oder anderes [...] (Hinz 2012: 33)

miteinbezieht. Ferner soll mit diesem Begriff die Kategorienbildung in gegensätzliche Gruppen, zum Beispiel Behinderte und Nicht-Behinderte, gesellschaftlich abgebaut werden (vgl.: ebd.), so dass es auch zu einem Abbau des Sonderstatus von Schülerinnen und Schülern mit Beeinträchtigung kommt (Wocken 2011: 72). Inklusion bezieht sich folglich auf gesamtgesellschaftliche Entwicklungen, die deutlich über den schulischen Kontext hinausgehen. Im schulischem Kontext wird der Begriff Inklusion jedoch, wenn es um den gemeinsamen Unterricht von beeinträchtigten und nichtbeeinträchtigen Kindern geht, in seiner Komplexität in der Regel auf den Heterogenitätsaspekt der „Beeinträchtigung“ reduziert. Wocken konstatiert

nicht eingegangen, sie sei jedoch der Vollständigkeit halber erwähnt (vgl. Frühauf 2012). 
daher, dass eine „inklusive Schule und eine inklusive Gesellschaft [...] große Utopien [sind], die wir schwerlich ohne jegliche Abstriche und ohne jegliche Zwischenaufenthalte erreichen können“ (ebd.: 81).

Der Begriff des „gemeinsamen Unterrichts“ scheint in dieser Situation eine neutrale oder politisch entschärfte Variante darzustellen, die zwischen den Begriffen der Integration und der Inklusion steht. So ist auf den Internetseiten des Ministeriums für Schule und Weiterbildung des Landes Nordrhein-Westfalen gemeinsamer Unterricht wie folgt beschrieben: „Im Gemeinsamen Unterricht [...] lernen Kinder und Jugendliche mit sonderpädagogischem Förderbedarf zusammen mit Kindern und Jugendlichen ohne sonderpädagogischen Förderbedarf in einer allgemeinen Schule.“ „Gemeinsamer Unterricht" scheint bewusst nicht von Inklusion getrennt zu werden, da Inklusion seinerseits durch die Verwendung des Begriffs des gemeinsamen Lernens definiert wird: „In einem inklusiven Schulsystem wird das gemeinsame Leben und Lernen von Menschen mit und ohne Behinderungen zur Normalform.“ Die Zitate zeigen, dass der Begriff des „gemeinsamen Unterrichts“ inhaltlich eine große Nähe zu Integration aufweist, während das Konzept genutzt wird, um Inklusion zu definieren.

Zusammenfassend lässt sich festhalten, dass eine klare Abgrenzung der Begriffe Integration, Inklusion und gemeinsamer Unterricht generell nur schwerlich gelingt. Auf der einen Seite zeigt die begriffliche Unschärfe zukünftiges konzeptionelles Entwicklungspotential, erschwert jedoch auf der anderen Seite die fachliche und interdisziplinäre Diskussion, da auf eine vorangehende Verständigung über die Begrifflichkeiten nicht verzichtet werden kann.

Im Folgenden wird der Begriff der Integration verwendet, um zum Ausdruck zu bringen, dass sich die folgenden Überlegungen ausschließlich darauf beziehen, wie beeinträchtigte Schülerinnen und Schüler in den Englischunterricht der Sekundarstufe I integriert werden können. Dabei erschwert das historisch gewachsene mehrgliedrige deutsche Schulsystem integrative / inklusive Prozesse, da Integration und Inklusion dem Ziel, möglichst homogene Lerngruppen bilden zu können, zuwider laufen. So wird Integration hier in Anlehnung an Wocken (2011) als notwendige Entwicklungsstufe auf dem Weg zu einem, im eigentlichen Sinn, inklusiven Schulsystem verstanden, das sich von unserem heutigen Schulsystem strukturell deutlich unterscheiden müsste. 


\section{2. ,Forschungsstand' für den integrativen Englischunterricht}

Das Forschungsfeld der integrativen (Fach)didaktik ist noch nicht etabliert, und folglich liegen bisher kaum empirische Arbeiten vor. Jedoch gibt es sowohl in benachbarten Disziplinen als auch in der Fachdidaktik Ansätze, die für die Gestaltung von integrativem Englischunterricht aufgegriffen werden können.

Im Rahmen der Förderpädagogik gibt es bereits Ideen zur Gestaltung von Englischunterricht unter Berücksichtigung des jeweiligen Förderschwerpunktes (z.B. Bartosch \& Rohde 2014). Je nach Zusammensetzung der integrativen Klasse in der Sekundarstufe der Regelschule könnten diese Ansätze im Rahmen der individuellen Förderung der Schülerinnen und Schüler mit sonderpädagogischem Förderbedarf genutzt werden, sie könnten aber auch für die gesamte Klasse positiv sein. Im Hinblick auf wirklich integrativen Unterricht sollte eine Organisationsform, in der sich die Schülerinnen und Schüler mit sonderpädagogischem Förderbedarf in den meisten Fächern in äußerer Differenzierung befinden, vermieden werden, da eine solche Regelung nicht den Anspruch an ,gemeinsames' Lernen erfüllt.

Sowohl die Grundschuldidaktik als auch die Fachdidaktik Englisch haben Überlegungen für den (integrativen) Englischunterricht in der Grundschule entwickelt (vgl.: Börner 2002; Kirchhoff 2012; Klippel 2000). Hier wäre zu überlegen, inwieweit sich die Arbeitsweisen des (integrativen) Englischunterrichts aus der Grundschule, zum Beispiel der Primat der Mündlichkeit, eine eher spielerische Herangehensweise oder eine stärkere Handlungsorientierung, Gewinn bringend in die Sekundarstufe I übertragen lassen. An diesem Punkt setzt Degen (1999) an, indem sie diskutiert, inwieweit binnendifferenzierende Methoden aus dem Grundschulbereich wie zum Beispiel das Bereitstellen von Lernhilfen, Planarbeit oder ein spielerischer Zugang auch in der Sekundarstufe verwendet werden können.

Auf methodischer Ebene ist damit eine Beziehung zum fachdidaktischen Umgang mit Heterogenität geknüpft. Trautmann diskutiert unter den Stichworten „Differenzierung von oben“ und „Differenzierung von unten“ (Trautmann 2010: 6) verschiedene Formen von Differenzierung für den Englischunterricht, stellt aber zugleich im Rückgriff auf die DESI-Studie fest, dass in der unterrichtlichen Praxis der Sekundarstufe Binnendifferenzierung wohl eine geringere Rolle spielt und kaum empirisch erforscht ist. 
Darüber hinaus spielt Heterogenität in der Fachdidaktik Englisch traditionell eine bedeutsame Rolle unter dem Stichwort der Lernschwäche und der lernschwachen Schülerinnen und Schüler (Butzkamm 2007; Hellwig 1984; Piepho 1983). Bei Berücksichtigung früherer Überlegungen und empirischer Erprobungen könnte ein erneuter Diskurs über Lernschwäche geführt werden, der auch die Diskussion über integrativen Unterricht in die Überlegungen und empirischen Versuche einbezieht.

Die bisher angesprochenen methodischen Aspekte, Fragestellungen und Forschungsansätze bedürfen gerade für den integrativen Englischunterricht in der Sekundarstufe I einer kritischen Hinterfragung, da sie nicht unbesehen auf das neue Handlungsfeld des integrativen Englischunterrichts angewandt werden können. Es ist daher zu fragen: Wie lassen sich methodische Konzepte und unterrichtliche Vorgehensweisen im Englischunterricht so gestalten, dass Integration erleichtert wird? Kann Englischunterricht das Thema Integration in seinen Unterrichtsinhalten aufgreifen, und zwar vergleichbar der Art und Weise, wie in den zurückliegenden Jahren die Frage der Gendergerechtigkeit in den Lehrwerken aufgearbeitet wurde (vgl. Heuer \& Müller 1973)?

Hier setzen gegenwärtig literaturdidaktische Vorschläge an, die im Diskurs um Interkulturalität und Fremdverstehen eine inhaltliche Beziehung zwischen Integration und dem Fremdsprachenunterricht herstellen (Küchler \& Roters 2014). Auch Thaler (2012) schlägt in seinen Ausführungen zu „The Absolute True Diary of a Part-Time Indian“ vor, den Umgang mit Beeinträchtigung anhand des Protagonisten zu thematisieren. Unter dem Leitziel „interkulturelle kommunikative Kompetenz “ wird demnach Fremdverstehen im Rahmen der unterrichtlichen Auseinandersetzung mit Literatur auf Menschen mit einer Beeinträchtigung ausgedehnt, wobei durch Perspektivwechsel ein tieferes Verstehen angestrebt wird (Bredella 2000; Byram 1997).

Obwohl die vorgestellten Ansätze und Überlegungen sicherlich Orientierungshilfen und gute Ausgangspunkte für weitere Überlegungen sind, reichen sie nicht aus, um das komplexe und spezielle Szenario des integrativen Englischunterrichts zu beschreiben und inhaltliche sowie methodische Ansprüche des Faches im Hinblick auf besonders heterogene Lerngruppen zusammenzubringen. So fassen denn auch Küchler \& Roters den gegenwärtigen Forschungsstand im Hinblick auf die Fremdsprachendidaktik folgendermaßen zusammen: „[...] ein Fundus an empirischen Studien - insbesondere 
bezogen auf die sprachlichen Unterrichtsfächer - liegt aber noch nicht vor. Auch Handreichungen und Hinweise für die konkrete Umsetzung im Englischunterricht sind rar“ (Küchler \& Roters 2014: 234).

\section{Methodische Neuausrichtung im integrativen EU: Muttersprache als kommunikative Lernhilfe und TBLT als ein möglicher binnendifferenzierender Ansatz}

Da noch keine spezifischen Forschungsergebnisse zum integrativen Englischunterricht vorliegen, sollte überlegt werden, wie bereits etablierte Ansätze des Fremdsprachenunterrichts für einen integrativen Unterricht aufgegriffen und modifiziert werden können.

So ist zu fragen, ob nicht Butzkamms Ansatz, die Mutter- und Schulsprache Deutsch als Lernhilfe in den Fremdsprachenlernprozess zu integrieren, die gegenwärtig vorherrschende Meinung zur methodischen Gestaltung des Unterrichts sinnvoll neu beleben könnte (Butzkamm 2007); seiner Vorstellung entsprechend wird die deutsche Sprache dabei insbesondere für schwächere Schülerinnen und Schüler förderlich eingesetzt. Es geht nicht um Übersetzung oder Mediation, sondern um ein inhaltlich bedeutsames Lernen von kommunikativen Sprachmitteln in einem zeitlich begrenzten Rahmen: Die deutsche Sprache wird als Gedankengeber oder inhaltlicher Impuls eingesetzt, um mit zunehmend größerer Sicherheit und Schnelligkeit Gedanken auf Englisch zu versprachlichen. Es geht um systematischen und gelenkten Sprachaufbau mit dem Ziel, eine Steigerung der kommunikativen Ausdrucksfähigkeit zu erreichen. Gerade schwächere Schülerinnen und Schüler können von solch einem Sprachtraining profitieren, da sie bei der Entwicklung von kommunikativen Aussagen gedanklich entlastet werden, um sich vermehrt auf Formulierarbeit konzentrieren zu können. Die vorliegenden empirischen Versuche von Butzkamm sind vielversprechend. In der DESI-Studie wurde passend zu dieser methodischen Konzeption empirisch nachgewiesen, dass die Entwicklung von sprachlich funktionalen Kompetenzen im Englischunterricht gefördert wird, wenn im Unterricht eine Engführung auf Sprache erfolgt, d.h. wenn Formulierarbeit geleistet wird (Helmke, Helmke, Schrader, Wagner, Nold \& Schröder 2008: 361).

Es ist ferner die Möglichkeit zu diskutieren, in welcher Weise sich TaskBased-Language-Teaching (TBLT) binnendifferenzierend in integrativen 
Klassen einsetzen lässt, da hier bei der Bearbeitung einer kommunikativen Aufgabe (Task) Schülerinnen und Schüler mit unterschiedlichen Leistungsanteilen im Task Cycle zum abschließenden Ergebnis der Aufgabenstellung beitragen können (Ellis 2004; Willis \& Willis 2007). Nach Willis \& Willis (1996) besteht aufgabenorientierter Unterricht aus drei Phasen, der Pretask, dem Task Cycle an sich, in dem die Schülerinnen und Schüler die Phasen des Planens, der Bearbeitung der Task und der Präsentation der Ergebnisse (outcome) durchlaufen, und der Post-task. Die Pre-task dient der Hinführung zum Task Cycle, wobei dieser zentrale Teil des TBLT Ansatzes meist in Kleingruppen oder Partnerarbeit durchgeführt wird, um eine größtmögliche Schüleraktivität entsprechend dem Grundgedanken des Ansatzes zu gewährleisten. Im Task Cycle findet dementsprechend die entscheidende kommunikative Arbeit der Schülerinnen und Schüler statt bis hin zur Präsentation ihrer Ergebnisse, und in der Post-task wird schließlich über das Gelingen der Schüleraktivitäten im Umgang mit einer Task reflektiert und es wird unter der Überschrift focus on form auch auf die Frage der sprachlichen Korrektheit sowie der Angemessenheit der Äußerungen eingegangen.

Abb. 1: Konkretisierung des Task-Cycle für den integrativen Englischunterricht (eigene Abbildung in Anlehnung an Willis \& Willis (1996: 38))

Konkretisierung des Task-Cycle für den integrativen Englischunterricht

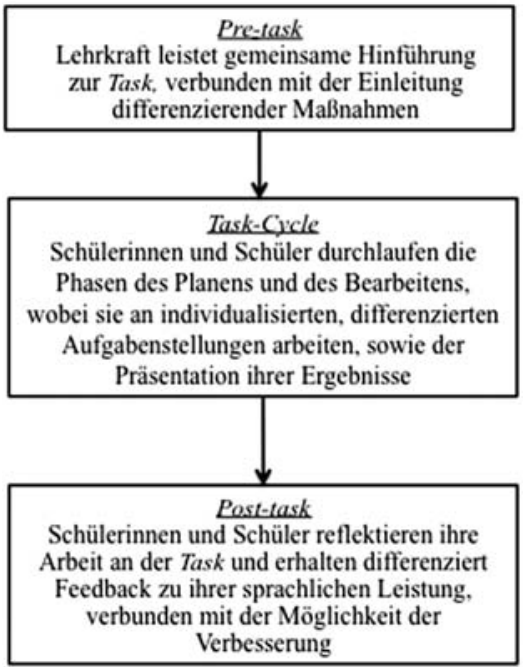


Obwohl dieses Lernarrangement auf den ersten Blick klar strukturiert wirkt, ist seine Komplexität nicht zu unterschätzen. Diese könnte Schülerinnen und Schüler, die Schwierigkeiten haben, komplexe Vorgänge zu verarbeiten oder Unsicherheiten auszuhalten, überfordern. Die betreffenden Schülerinnen und Schüler müssten mit sprachlich einfachen und graphisch klar strukturierten Arbeitsblättern, die es ihnen erlauben, ihre Position im Arbeitsprozess zu markieren, um so zu erkennen, welches der nächste Arbeitsschritt ist, relativ eng begleitet werden. Die Arbeitsblätter müssten für die konkrete Task und entsprechend der individuellen Voraussetzungen der Schülerinnen und Schüler gestaltet werden.

Der Ansatz des Task-based-Language-Teaching erscheint in besonderem Maße mit den Ansprüchen an gemeinsames Lernen und gleichzeitiger individueller Förderung aller Schülerinnen und Schüler übereinzustimmen. Pre- und Post-task ermöglichen einen gemeinsamen Einstieg und ebenso einen gemeinsamen Abschluss, in dem es zuvor durch die Bearbeitung von differenzierten, den Fähigkeiten der Schülerinnen und Schüler angepassten Tasks, möglich ist, dass auch Schülerinnen und Schüler, die aufgrund einer Beeinträchtigung über geringere sprachliche Kompetenzen verfügen, Ergebnisse präsentieren können. Die Passung des Task-based-Language-Teaching zu den Anforderungen des integrativen Unterrichts ergibt sich also aus einer mehrfach differenzierten Task. Der Ansatz ermöglicht, dass Schülerinnen und Schüler gemeinsam, das heißt am gleichen Gegenstand, lernen oder zum gleichen Thema arbeiten, aber dies auf verschiedenen, ihren individuellen Fähigkeiten angepassten Niveaus tun.

Ein praktisches Beispiel ist die Produktion eines Hörspiels zu einer Kurzgeschichte oder einer Szene. Anregungen zu möglichen Texten, die auch schwachen Schülern zugänglich sind, finden sich zum Beispiel bei Oxford Bookworms. Denkbar ist eine Arbeit mit Sherlock Holmes Short Stories, die mit Niveau A2 / B1 des Common European Framework angegeben sind (West 2008). Das detektivische Talent Sherlock Holmes spricht Kinder und Jugendliche seit Generationen an und hat in den letzten Jahren durch die Kinofilme des Regisseurs Guy Ritchie $(2009,2011)$ und die BBC-Serie Sherlock (Gatiss \& Moffat 2010-2014) neue Popularität erhalten.

Dabei ist zu bedenken, dass trotz der bereits vorgenommenen sprachlichen Vereinfachung des Textes eine weitere Kürzung des Textes durch die Lehrkraft für einzelne Schülerinnen und Schüler notwendig sein kann. Um 
allen Schülerinnen und Schülern einen Textzugang zu ermöglich, kann der Text lexikalisch und syntaktisch sowie inhaltlich vereinfacht werden, und es können Zusammenfassungen und Zwischenüberschriften eingefügt werden. Gerade für Schülerinnen und Schüler, denen der Umgang mit schriftlichen Texten schwer fällt, ist eine Bebilderung zu erwägen. Als Pre-task ist ein gemeinsamer Beginn im Sinne einer klassischen Pre-reading Task denkbar, zum Beispiel Brain-Storming zu einem zur Geschichte passendem Bild. Die anschließende While-reading Phase bedarf ebenfalls individueller Unterstützung. Nach Rezeption des Textes und Sicherung des Textverständnisses erfolgt die eigentliche Task, die Produktion des Hörspiels.

Dabei ist zuerst die Zusammensetzung der Arbeitsgruppen zu bedenken. Damit diese dem Anspruch integrativen Unterrichts und gemeinsamen Lernens entsprechen, sollten die Gruppen möglichst heterogen zusammengesetzt sein. Organisatorisch bedingt dies eine vorherige Gruppeneinteilung durch die Lehrkraft. Die konkrete Aufgabenverteilung innerhalb der Gruppe sollte dann, dem Ansatz des TBLT folgend, von den Schülerinnen und Schülern eigenständig ausgehandelt werden. In integrativen Klasen ist hier eine stärkere Beteiligung der Lehrkräfte in der Regel erforderlich. So sind für die Produktion eines Hörspiels verschiedene Sprecherrollen und ein Erzähler zu besetzen. Die Auswahl des Textteils der Kurzgeschichte kann idealerweise von der Gruppe geleistet werden; dies hat möglicherweise schon eine Reduktion des Sprechumfangs für einige Rollen zur Folge. Die Gruppe kann nämlich Sprechanteile von Rollen kürzen, aufteilen oder Sprechanteile vereinfachen. Ziel sollte dabei sein, dass sich jeder Sprecher seiner Rolle gewachsen fühlt. Folglich werden die Rollen im Hinblick auf individuelle Leistungsfähigkeit sowohl im Umfang als auch in ihrer sprachlichen Anforderung stark variieren. Denkbar ist es weiterhin für sehr schwache oder stark beeinträchtigte Schülerinnen und Schüler Rollen zu kreieren, deren Sprechumfang auf ein Minimum, eventuell einen kurzen Satz, reduziert ist. Weitere Differenzierungsmöglichkeiten bietet die Ausführung von Hintergrundgeräuschen oder die Auswahl stimmungserzeugender Hintergrundmusik. Durch diese Maßnahmen würden sprachlich schwache Schülerinnen und Schüler aktiv an der Produktion des Hörspiels beteiligt. Auf der einen Seite ist der zielsprachige Kontakt bei der Übernahme dieser Aufgaben deutlich geringer, auf der anderen Seite ermöglicht dieses Vorgehen Schülerinnen und Schülern mit sprachlichen Schwächen Teil der Gruppe zu sein 
und eine eigenständige Leistung erbringen zu können. Die motivationalen und sozialen Aspekte sind dabei nicht zu unterschätzen.

Die Aufnahme des Hörspiels kann zum Beispiel über die Mobiltelefone der Schülerinnen und Schüler oder über Schul-Laptops mit integriertem Mikrophon erfolgen. In Anlehnung an die differenzierte Task sollten auch die Dokumentation des Arbeitsprozesses sowie die Peer-Assessment-Bögen, die während der Vorstellung der Produkte zum Einsatz kommen, entsprechend differenziert sein. Diese können von kriteriengeleiteter Kommentierung in ganzen Sätzen oder Stichworten bis zum Ankreuzen von Emoticons reichen.

$\mathrm{Zu}$ bedenken ist bei der Nutzung von TBLT in integrativen Klassen, dass sich die Rollen „starker“ und „schwacher“ Schüler nicht verfestigen dürfen, da diese Aufteilung nicht nur das Prinzip der Integration unterläuft, sondern auch negative Auswirkungen auf das Sprachlern-Selbstkonzept der Schüler haben kann. Im Schutz des Klassenraumes und in Kleingruppen können sprachlich schwächere Schüler vorsichtig und gezielt aus ihrer Komfortzone geholt werden, da ihnen hier sprachliche Erfolgserlebnisse Selbstvertrauen geben, ihre Motivation stärken und so ihren Lernfortschritt sichern. Allerdings ist einzuräumen, dass diese Erwartung sich nicht in jedem Fall erfüllen wird. Die Gefahr eines Auseinanderdriftens der Schülergruppen bleibt bestehen.

\section{Umgang mit Bildungsstandard und Leistungsbewertung (Assessment) im integrativen Englischunterricht}

Aufgrund der individuellen Sprachlernvorrausetzungen der Schülerinnen und Schüler ist davon auszugehen, dass die sprachlichen Kompetenzen in integrativen Klassen breiter angelegt sind als in Regelklassen. In Hinblick auf die von der Kultusministerkonferenz beschlossenen Bildungsstandards für die erste Fremdsprache kann von Beginn an im Englischunterricht mit einer großen Kompetenzspanne gerechnet werden, die sich im Verlauf des Bildungsgangs von A1 bis zu den geforderten höheren Kompetenzniveaus B1 oder sogar B2 im Bereich Leseverstehen erstrecken (vgl. Kultusministerkonferenz 2003: 11f.). Diese Kompetenzspannweite zu berücksichtigen, stellt die Lehrkräfte vor die Herausforderung, Unterricht so zu planen und zu gestalten, dass Schülerinnen und Schüler auf der einen Seite entsprechend ihren Kompetenzen individuell gefördert werden und auf der anderen Seite gemeinsam an einem Unterrichtsgegenstand arbeiten. Die mehrere Kompetenzstufen umfassende 
Unterrichtsgestaltung setzt sich folglich auch in der Leistungsüberprüfung fort und verlangt nach der Erstellung differenzierter Tests, die sich an den individuellen Fähigkeiten der Schülerinnen und Schüler orientieren. In diesem Zusammenhang sind neben eher traditionellen Klassenarbeiten auch alternative Formen der Beurteilung und Bewertung zu erwägen. Portfolios weisen in ihrem prozessdokumentierenden Charakter eine hohe Passung zum Ansatz des TBLT auf und ermöglichen neben einer schriftsprachlichen auch eine kreative Auseinandersetzung mit dem Lerngegenstand, in der individuellen Lernvoraussetzungen Rechnung getragen werden kann. Auch die bereits eingeführten mündlichen Prüfungen erlauben eine Differenzierung und geben schriftsprachlich schwächeren Schülerinnen und Schülern die Gelegenheit, ihre kommunikativen Fähigkeiten mündlich stärker zur Geltung zu bringen und zu entwickeln.

Heterogenität der sprachlichen Kompetenzen der Schülerinnen und Schüler ist unterrichtliche Realität. Jedoch wird auch deutlich, dass ein Umgang mit stark variierenden sprachlichen Kompetenzen erhebliche Mehrarbeit für die Lehrkräfte bedeutet, wenn gleichzeitig der Anspruch an die individuelle Förderung aller Schülerinnen und Schüler erfüllt werden soll. Ohne Team-Teaching zusammen mit förderpädagogischen Fachkräften ist ein verantwortlicher Umgang mit Heterogenität wohl kaum zu leisten.

\section{Forschungsdesiderata des integrativen Englischunterrichts}

Da bisher kaum empirische Studien zum integrativen Fremdsprachen- oder Englischunterricht vorliegen, sind die Forschungsdesiderata im Bereich des integrativen Unterrichts zahlreich, wobei sich zur Systematisierung die Desiderata in Bezug auf die am Unterricht beteiligten Personengruppen abbilden lassen.

\section{Abb. 2: Forschungsdesiderata im integrativen Englischunterricht}

\section{Forschungsdesiderata im integrativen Englischunterricht}

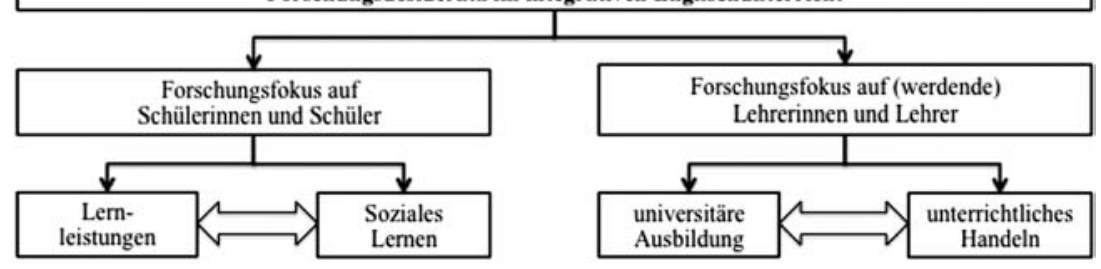


Im Hinblick auf eine effektive Gestaltung integrativen Englischunterrichts wäre es wichtig, Informationen über die Lernleistung von Schülerinnen und Schülern zu erhalten. Dabei wäre im Rahmen eines quantitativen Vorgehens ein Vergleich zwischen dem Lernzuwachs von Schülerinnen und Schülern mit und ohne sonderpädagogischen Förderbedarf denkbar. Aber auch ein Vergleich von Lernleistungen zwischen integrativen und nicht integrativen Klassen, eventuell bezogen auf isolierbare sprachliche Kompetenzen, könnte Einsichten in die Potentiale integrativen Englischunterrichts geben. Ebenfalls aus Schülerperspektive zu erforschen wäre die affektive / soziale Seite. Erkenntnisse zu der Frage sind nötig, welchen Einfluss das gemeinsame Lernen von sehr heterogenen Schülergruppen auf die Motivationen und Einstellungen haben. Kann die Gefahr von Unter- oder Überforderung mit allen Konsequenzen für das Selbstbild der Lerner bewältigt werden?

Besonders relevant für integrativen Englischunterricht wäre es, Aussagen über das Verhältnis von fachlichem und sozialem Lernen im integrativen Englischunterricht machen zu können. Eine entsprechende Studie wäre naturgemäß mit einem großen forschungsmethodologischen Aufwand verbunden, der nur interdisziplinär zu leisten wäre. Es liegen ebenfalls keine Studien zu den Kompetenzen und Handlungsweisen von Lehrerinnen und Lehrern im Englischunterricht vor. Auf universitärer Ebene haben Amrhein und Bongartz (2014) mit der Befragung der Teilnehmerinnen und Teilnehmer der Vortragsreihe „Im Dialog der Disziplinen. Englischdidaktik Förderpädagogik - Inklusion“ einen Anfang gemacht. Größer angelegte Befragungen dieser Art könnten Impulse für die konzeptionelle Arbeit an universitären Curricula geben, die zur Zeit für die Studiengänge der Regelschulen noch eine integrative / inklusive Ausrichtung vermissen lassen. Um jedoch auf universitärer Ebene Handlungskompetenzen für den integrativen Fremdsprachenunterricht anbahnen zu können, sind genauere Informationen über die unterrichtspraktische Realität im integrativen Englischunterricht notwendig. Diese könnten mit Hilfe qualitativer Ansätze angegangen werden, die sich auf die Lehrerinnen und Lehrer und ihr unterrichtliches Handeln und auf schulstrukturelle Voraussetzungen konzentrieren.

\section{Literaturverzeichnis}

Amrhein, Bettina \& Bongartz, Christiane M. (2014), Diversity and Inclusion in Second and Foreign Language Learning - Chancen für die 
LehrerInnenbildung. In: Bartosch, Roman \& Rohde, Andreas (Hrsg.) (2014), Im Dialog der Disziplinen. Englischdidaktik - Förderpädagogik - Inklusion. Trier: Wissenschaftlicher Verlag Trier, 25-44.

Bartosch, Roman \& Rohde, Andreas (Hrsg.) (2014), Im Dialog der Disziplinen. Englischdidaktik - Förderpädagogik - Inklusion. Trier: Wissenschaftlicher Verlag Trier.

Börner, Otfried (2002), Englischunterricht in der Primarstufe der Förderschule. Fremdsprachen Frühbeginn, 4: 02, 5-10.

Bredella, Lothar; Meißner, Franz-Joseph; Nünning, Ansgar \& Rösler, Dietmar (Hrsg.) (2000), Wie ist Fremdverstehen lehr-und lernbar? Tübingen: Narr.

Butzkamm, Wolfgang (2007), Schwache Englischleistungen - woran liegt's? Glanz und Elend der Schule oder die Wirklichkeit des Fremdsprachenschülers. Zeitschrift für Interkulturellen Fremdsprachenunterricht 12: 1 [Online: https://zif.spz.tu-darmstadt.de/ig-12-1/docs/ Butzkamm.pdf 12.03.2015].

Byram, Michael (1997), Teaching and Assessing Intercultural Communicative Competence. Clevedon: Multilingual Matters.

Degen, Sabrina (1999), Integration Im Englischunterricht. Neuwied, Kriftel, Berlin: Luchterhand.

Ellis, Rod (2003), Task-based language learning and teaching. Oxford: Oxford University Press.

Frühauf, Theo (2012), Von der Integration zur Inklusion - ein Überblick. In: Hinz, Andreas; Körner, Ingrid \& Niehoff, Ulrich (Hrsg.) (2012), Vor der Integration zur Inklusion. Grundlagen - Perspektiven - Praxis. Marburg: Lebenshilfe-Verlag, 11-32.

Gatiss, Mark \& Moffat, Steven (Creators) (2010-2014), Sherlock. BBC.

Hellwig, Karlheinz \& Sauer, Helmut (Hrsg.) (1984), Englischunterricht für alle. Beiträge zur Didaktik und Methodik des Englischunterrichts in Lerngruppen mit schwierigen Voraussetzungen. Paderborn: Schöningh. Helmke, Tuyet; Helmke, Andreas; Schrader, Friedrich-Wilhelm; Wagner, Wolfgang; Nold, Günter \& Schröder, Konrad (2008), Die Videostudie des Englischunterrichts. In: DESI-Konsortium (Hrsg.) (2008), Unterricht und Kompetenzerwerb in Deutsch und Englisch. Weinheim und Basel: Beltz, 345-363. 
Heuer, Helmut \& Müller, Richard M. (Hrsg.) (1973), Lehrwerkkritik - ein Neuansatz. Dortmund: Lensing.

Hinz, Andreas (2012), Inklusion - historische Entwicklungslinien und internationale Kontexte. In: Hinz, Andreas; Körner, Ingrid \& Niehoff, Ulrich (Hrsg.) (2012), Von der Integration zur Inklusion. Grundlagen Perspektiven - Praxis. Marburg: Lebenshilfe-Verlag, 33-52.

Kirchhoff, Petra (2012), Inklusion im Englischunterricht der Grundschule. Ein Anfang ist gemacht!. Praxis Fremdsprachenunterricht Englisch 09: 01, 4-6.

Klippel, Friederike (2000), Englisch in der Grundschule. Berlin: Cornelsen/ Scriptor.

Küchler, Uwe \& Roters, Bianca (2014), Embracing Everyone: Inklusiver Fremdsprachenunterricht. In: Amrhein, Bettina \& Dziak-Mahler, Myrle (Hrsg.) (2014), Fachdidaktik Inklusiv. Auf der Suche nach didaktischen Leitlinien Für den Umgang mit Vielfalt in der Schule. Münster \& New York: Waxmann, 233-248.

Kultusministerkonferenz, Beschlüsse (2003), Bildungsstandards für die erste Fremdsprache (Englisch / Französisch) für den mittleren Schulabschluss. [Online: http://www.kmk.org/fileadmin/veroeffentlichungen_ beschluesse/2003/2003 12 04-BS-erste-Fremdsprache.pdf. 10.03.2015].

Ministerium für Schule und Weiterbildung des Landes Nordrhein-Westfalen, Inklusion, [Online: https://www.schulministerium.nrw.de/docs/Schulsystem/Inklusion/index.html. 16.02.2015].

Ministerium für Schule und Weiterbildung des Landes Nordrhein-Westfalen, Integration, [Online:https://www.schulministerium.nrw.de/docs/Schulsystem/Schulformen/Grundschulen/Von-A-bis-Z/Gemeinsamer-Unterricht/ index.html. 16.02.2015].

Nunan, David (2004), Task-based language teaching. Cambridge: Cambridge University Press.

Piepho, Hans-Eberhard (1983), Englisch in Gruppen mit Leistungsschwierigkeiten. Schule beute 11, 22-25.

Ritchie, Guy (Regie) (2009), Sherlock Holmes. Warner Bros.

Ritchie, Guy (Regie) (2011), Sherlock Holmes. Spiel im Schatten. Warner Bros.

Thaler, Engelbert (2012), A Part-Time Indian - Literatur und Behinderung. Praxis Fremdsprachenunterricht Englisch 09: 01, 13-14. 
Trautmann, Matthias (2014), Heterogenität. (K)ein Thema in der Fremdsprachendidaktik. In: Köker, Anne; Romahn, Sonja \& Textor, Annette (Hrsg.) (2010), Herausforderung Heterogenität. Bad Heilbrunn: Klinkhardt, 52-64 [Online: http://www.bag-englisch.de/wp-content/uploads/2010/01/ Heterogenität-Trautmann.pdf. 10.03.2015].

West, Clare (Hrsg.) (2008), Sherlock Holmes Short Stories. Oxford Bookworms Library Stage 2. Berlin: Cornelsen Schulbuchverlage.

Willis, Dave \& Willis, Jane R. (2007), Doing task-based teaching. Oxford: Oxford University Press.

Willis, Dave \& Willis, Jane R. (1996), A Framework for Task-based Learning. Harlow: Longman.

Wocken, Hans (2011), Das Haus der inklusiven Schule. Baustellen - Baupläne-Bausteine. Hamburg: Feldhaus Verlag. 
Christiane M. Bongartz and Andreas Rohde - 978-3-653-97188-0

Downloaded from PubFactory at 01/11/2019 10:41:16AM

via free access 


\section{Jan Springob \\ Die ersten Schritte sind gemacht: Inklusiver Englischunterricht an einem Gymnasium - Herausforderung und Chance}

This article presents a first-hand report on the experiences made with the first inclusive cohort of inclusive EFL in the fifth grade of a grammar school in North Rhine-Westphalia. The author, a teacher-action-researcher, documented the first year of instruction in an inclusive classroom of 22 students, of which six were students with special needs (learning, emotional and social development, physical development). Teaching was focused on the acquisition of communicative skills rather than theoretical language knowledge, as exemplified by selected teaching techniques and tasks. The results of a comparative class test give insight in the learning development of the students taught inclusively compared to that of students taught in a non-inclusive setting. Successful inclusive instruction, as the author shows, crucially depends on extended resources, suitable teaching material, and individualized teaching and support.

\section{Einführung - die öffentliche Debatte}

Ich bin anders, so wie Du. Bildung steht uns allen zu. Dieser kurze Vers lachte die Kölner Bevölkerung im Sommer 2013 von riesigen Werbeflächen der Stadt an. Es handelt sich dabei um den Siegerslogan des Plakatwettbewerbs Alle inklusive, den die Dommetropole wenige Wochen zuvor gekürt hatte. Inklusion ist aktuell das bildungspolitische Thema in Deutschland. Menschen mit Behinderung, Förder- bzw. Unterstützungsbedarf werden als ganz „normaler“ Teil unserer Gesellschaft beworben: Vielfältigkeit als Gewinn, Andersartigkeit als Bereicherung - frei dem kölschen Motto: Jeder Jeck ist anders.

Diese landesweite Kampagne war eigentlich längst überfällig und in seinen Grundgedanken uneingeschränkt zu unterstützen. 2009 hat auch Deutschland die UN-Behindertenrechtskonvention Übereinkommen über die Rechte von Menschen mit Behinderungen ratifiziert (UN 2008). Alle Menschen haben folglich das Recht auf eine aktive Teilhabe am gesellschaftlichen Leben. Doch die Entscheidungen der Politik, die in der Öffentlichkeit 
medienwirksam aufbereitet und als gesetzt verbreitet werden, bringen in der täglichen Realität weitreichende Konsequenzen mit sich. So hat die Bestätigung der Konvention auch und vor allem für unser Bildungssystem erhebliche Folgen. Die Kultusministerkonferenz beschloss im Februar 2011 einen Entwurf zur Weiterentwicklung ihrer Empfehlungen zur sonderpädagogischen Förderung von 1994. Dieser Entwurf trägt nun die Überschrift Inklusive Bildung von Kindern und Jugendlichen mit Behinderungen in Schulen (KMK 2011). Folglich haben alle Schülerinnen und Schüler (SuS) das Recht in eine allgemeinbildende Schule ihrer Wahl zu gehen. Dies erfordert ein grundlegendes Umdenken in allen Schulformen, schließlich herrscht in Deutschland nach wie vor ein selektives Schulsystem vor, in dem vor allem Kinder und Jugendliche mit Förderbedarf in der Regel ausgeschlossen werden.

Gemeinsamen Unterricht von Kindern mit und ohne Förderbedarf gibt es in Nordrhein-Westfalen bereits an vielen Grund-, Gesamt- und Hauptschulen. Mit der Einführung des Rechtsanspruchs zum Besuch einer Regelschule auch für Kinder und Jugendliche mit Unterstützungsbedarf geraten nun auch die Gymnasien in den Blick und müssen sich den Anforderungen einer inklusiven Pädagogik stellen - trotz der Kritik, der Fragen und der Stolpersteine, die umfassend und öffentlich diskutiert werden. Zentral ist dabei die Frage, wie es möglich ist, sowohl SuS mit besonderem Förderbedarf als auch SuS mit einer gymnasialen Empfehlung - nicht zu vergessen all die SuS „dazwischen“ - in einer Schulform zu fördern, in der die Bewertung von Leistungen auf dem Prinzip der Selektion beruht? Wie kann inklusive Bildung für alle SuS gewinnbringend gelingen?

In diesem Artikel wird einleitend eine kurze Übersicht der zentralen Aspekte aus Forschung und Praxis, die eine inklusive Fremdsprachendidaktik stützen, gegeben. Losgelöst von einem Anspruch auf Vollständigkeit werden wichtige Erkenntnisse knapp präsentiert, um die wissenschaftliche, noch ausbaufähige, Grundlage für die unterrichtliche Praxis zu verdeutlichen. Darauf aufbauend werden konkrete Einblicke in die Praxis gewährt. Beispielhaft werden Englischstunden der Klassen 5 und 6 des Geschwister-SchollGymnasiums in Pulheim vorgestellt, wobei in diesem Kontext vor allem auf zwei Förderschwerpunkte - Lernen und Emotionale-soziale Entwicklung eingegangen wird. Auf der Grundlage erster Vergleichsarbeiten wird in einem dritten Schritt gezeigt, wie bedeutsam Diagnostik und Überprüfung sind. 
Dabei ist dieser Artikel als ein erster Zwischenbericht, eine Momentaufnahme aus der Praxis, zu verstehen. Viele weitere Untersuchungen und Erhebungen sollen und müssen folgen.

\section{Inklusive Fremdsprachendidaktik und -forschung: eine Annäherung}

Den einleitend skizzierten rechtlichen und politischen Vorgaben und Ideen steht nach wie vor ein sehr geringer Fundus an tatsächlichen Erfahrungen und konkreter wissenschaftlicher Forschung gegenüber. Bisher müssen Menschen, die in Deutschland im Bereich Inklusion und Fremdsprache arbeiten bzw. diesen erforschen - bildlich gesprochen - einen "Gemischtwarenladen“ aufsuchen und sich ihr Wissen und ihr Material aus verschiedenen „Abteilungen“ zusammenstellen. Preuss-Lausitz hat in einem kürzlich veröffentlichten Artikel die wissenschaftlichen Begleitungen der Wege zur inklusiven Schulentwicklung in den Bundesländern überblicksartig zusammengestellt (Preuss-Lausitz 2014). Erste Projekte wie das Rügener Inklusionsmodel in Mecklenburg-Vorpommern, oder die Evaluation der Moderatorenausbildung in Nordrhein-Westfalen sind vorhanden. ${ }^{1}$ Auch die Bielefelder Längsschnittstudie zum Lernen in inklusiven und exklusiven Förderarrangements (BiLieF) der Universität Bielefeld ist ein zentrales Projekt ${ }^{2}$; viele weitere müssen folgen.

Der Zweitspracherwerb ist zweifelsohne gut erforscht und umfassend dokumentiert (u.a. Bongaerts 2005; Cook \& Singleton 2014; Singleton 2005), doch liegt der Fokus hier eher auf „den allgemeinen und universalen Spracherwerbsprozessen “ (Küchler \& Roters 2014: 239). Konkrete Unterrichtsmaterialien sowie Handreichungen für guten Fremdsprachenunterricht gibt es ebenfalls zur Genüge (u.a. Bach \& Timm 2009; Weskamp 2007).

1 Preuss-Lausitz weist darauf hin, dass er sich vor allem auf die von den Bildungsministerien beauftragten und mitfinanzierten wissenschaftlichen Begleitungen bezieht. „Die darüber hinaus gehende Inklusionsforschung, die gerade in den letzten Jahren an den Hochschulen und Universitäten neuen Aufschwung genommen hat, kann hier nicht abgebildet werden. Eine entsprechende systematische Übersicht würde eine vollständige Abfrage bei allen sonder- und erziehungswissenschaftlichen Instituten erfordern“ (Preuss-Lausitz 2014: 4).

2 http://www.uni-bielefeld.de/inklusion/projekt.html (18. Juni 2015). 
Veröffentlichungen zu den Themenbereichen Heterogenität und individuelle Förderung bieten zusätzlich wichtige Ansätze für inklusiven Fremdsprachenunterricht (vgl. u.a. Übersicht Küchler \& Roters 2014: 235-240; Sturm 2013).

Doch gerade eine gewinnbringende Verbindung aus Wissenschaft und Praxis fehlt an vielen Stellen noch; der inklusive Fremdsprachenunterricht für SuS mit Förderbedarf ist bisher kaum erschlossen (Köpfer 2014: 158; Küchler \& Roters 2014: 235/236). „Wir wissen noch viel zu wenig über Fremdspracherwerbsprozesse von Kindern mit Förderbedarf. Weiterhin ist noch nicht bekannt, welche Methoden im Englischunterricht bei diesen Schülern wirksam sind “ (Rohde \& Schick 2012: 4). Wenig überraschend ist, dass Förderbedarfe - diagnostiziert oder nicht - die Möglichkeiten einzelner SuS, eine Fremdsprache zu lernen, eventuell einschränken oder erschweren.

Listening, speaking, reading, and writing are key modalities in a foreign language classroom. Since a learning disability may interfere with the study of language, instructors must be aware of how various learning disabilities may affect students' classroom performance. (Lazda-Cazers \& Thorson 2008: 107)

Vor allem für Gymnasien in Deutschland - die Schere zwischen leistungsstarken und leistungsschwachen SuS könnte nicht größer sein - gibt es bisher so gut wie keine konkreten Studien; „fächerbezogene Begleitprojekte inklusiver curricaler und methodischer Art" fehlen (Preuss-Lausitz 2014: 12).

Dies ist umso bedauerlicher, als ein guter inklusiver Unterricht sich nicht allgemein, sondern im jeweiligen Fach - und mit den jeweiligen Fachlehrkräften - realisiert. Fächerspezifische Forschungsfragen könnten sich sowohl auf die (Mindest)Lernziele und ihre individuelle Passung als auch auf methodische, kommunikative und bewertende Aspekte beziehen. Die Fachdidaktik- und FachwissenschaftsLehrstühle der Universitäten und Hochschulen könnten, in Zusammenarbeit mit der allgemeinen Schulpädagogik und der Sonderpädagogik, kompetente Auftragnehmer sein. (Preuss-Lausitz 2014: 12)

Einzelne Forscher betonen, dass viele Kinder und Jugendliche mit Beeinträchtigungen an Regelschulen eigentlich und insgesamt besser gefördert werden - belegt durch nationale und internationale Studien. ${ }^{3}$ Klaus Klemm

3 Prof. Dr. Clemens Hillenbrand weist in seinem Vortrag Inklusion und Gymnasium. Rechtliche Aspekte, wissenschaftliche Erkenntnisse (Kassel, 6. Februar 2012) auf die Problematik hin, dass erstens deutsche Befunde international kaum vorkommen und zweitens bestehende internationale Befunde in 
beispielsweise kommt in seiner Studie zu dem folgendem, eher allgemeinen Ergebnis:

Kinder mit besonderem Förderbedarf, die im Gemeinsamen Unterricht (GU) mit Kindern ohne Förderbedarf lernen und leben, machen im Vergleich deutlich bessere Lern- und Entwicklungsfortschritte. Zudem profitieren auch die Kinder ohne Förderbedarf vom Gemeinsamen Unterricht, indem sie höhere soziale Kompetenzen entwickeln, während sich ihre fachbezogenen Schulleistungen nicht von den Leistungen der Schülerinnen und Schüler in anderen Klassen unterscheiden. (Klemm 2009: 4)

Lazda-Cazers \& Thorson betonen, dass SuS mit Lernschwierigkeiten eine Fremdsprache erlernen können - auch wenn sie dabei eventuell weniger erfolgreich sind als Menschen ohne Unterstützungsbedarf (Lazda-Cazers \& Thorson 2008: 111). Sie verweisen darauf, dass vor allem das Alter eine Rolle spielt:

The exposure to language must happen early in life. This does not mean that it is impossible to master a foreign language beyond the window of opportunity. [...] However, after the onset of puberty, language learning requires more effort. (Lazda-Cazers \& Thorson 2008: 113)

Die Frage, die immer wieder diskutiert und erforscht wird, ist, ob es beim Zweitspracherwerb einen Zeitpunkt im Leben eines Menschen gibt, ab dem er oder sie die entsprechende neue Sprache nicht mehr „perfekt“ erlernen kann. ${ }^{4}$ Für SuS mit einer Lernschwäche oder einem Förderschwerpunkt, wie für viele SuS insgesamt, geht es aber wohl nicht um eine möglichst perfekte Beherrschung der Fremdsprache. Es geht um den Erwerb einer kommunikativen Kompetenz, die es ihnen ermöglicht, grundlegende Belange in der Fremdsprache verstehen und ausdrücken zu können.

Deutschland bisher nicht ausreichend rezipiert werden. International gibt es jedoch eine Auswahl an Studien, die für die inklusive Arbeit an Gymnasien in Deutschland hilfreich sein könnten (vgl. u.a. Lindsay (2007); Millrood (2002); Myklebust (2006)).

4 Cook \& Singleton (2014) gehen ebenfalls intensiv auf die sogenannte Critical Period Hypothesis ein. Das Alter scheint eine Rolle zu spielen - wie bei allen Lernprozessen - doch gibt es Studien, die belegen, dass auch erwachsene Menschen eine Fremdsprache erlernen konnten bzw. können (Cook \& Singelton 2014: 26). Darüber hinaus wird in vielen neueren Veröffentlichungen darauf hingewiesen, dass neben dem Alter auch andere Faktoren wie Motivation, Ausdauer oder Lernumgebung eine Rolle spielen (ebd.: 33). 
„Wichtigstes Bildungsziel des Englischunterrichts muss die kommunikative Kompetenz sein. Dies bedeutet im Wesentlichen das Vermögen, lebensweltliche Situationen sprachhandelnd bewältigen zu können “ (Haß 2014: 51). Klaus Hinz weist schon 1991 darauf hin, dass viele SuS, die eine Hauptschule besuchen, große Schwierigkeiten haben, eine Fremdsprache erfolgreich zu lernen - aus ganz unterschiedlichen Gründen. Genannte Auffälligkeiten im Lern- und Leistungsverhalten (z.B. ein verlangsamtes Lern- und Lesetempo, ein nur wenig entwickelter Leistungsanspruch oder eine schwache Merkfähigkeit) sind keine ausschließlich an Haupt- oder Förderschulen anzutreffenden Phänomene - und das nicht erst seit der Debatte um Inklusion. Die Schülerschaft, auch an Gymnasien, wird bunter und die Schulen müssen sich diesen Herausforderungen stellen (Hinz 1991: 195/196). „Foreign language learning opens new doors and opportunities for all students. Students with learning disabilities can become successful language learners given the right learning atmosphere and conditions" (Lazda-Cazers \& Thorson 2008: 126).

Klar muss in diesem Zusammenhang sein, dass jede Schülerin und jeder Schüler unterschiedlich ist. Auch eine Gruppe von SuS mit ein und demselben Förderbedarf stellt eine heterogene Gruppe dar. Schüler A hat beispielsweise Probleme mit der Aussprache, Schülerin B mit der Syntax, Schüler C mit dem Lang- oder Kurzzeitgedächtnis und Schülerin D mit dem Leseverständnis. So muss es darum gehen, individuell auf den jeweiligen Schüler und die jeweilige Schülerin zugeschnittene Unterstützungsmöglichkeiten zur Verfügung zu stellen.

Typical accommodations include using alternative assessment techniques, allowing the students extra time during a test, tolerating poor spelling and/or pronunciation, allowing the use of a spell checker for written work, providing supplemental tutoring, offering modified classes for students with learning disabilities [...]. (Lazda-Cazers \& Thorson 2008: 113)

Das ist eine Herausforderung an Schulen ganz allgemein und an Lehrerinnen und Lehrer im Besonderen. Doch kommen nicht erst mit dem Gedanken der Inklusion neue Impulse in das Bildungswesen. „In jeder Schule gibt es [...] vielfältige Praktiken, die zumindest inklusives Potential haben und versuchen, auf die vorhandene Vielfalt der Schülerschaft besser einzugehen“ (Klein-Landeck 2014: 11). Gleichzeitig kann Inklusion so auch eine Chance sein. 


\section{Gemeinsam und miteinander - ein Blick in die Praxis}

Das Geschwister-Scholl-Gymnasium in Pulheim ${ }^{5}$ hat sich auf den Weg gemacht und zum Schuljahr 2012/13 eine erste inklusive Klasse eingerichtet. Neben 16 sogenannten Regelschülerinnen und -schülern werden in dieser Klasse sechs SuS mit Förderbedarf unterrichtet. Drei SuS werden im Förderschwerpunkt Lernen zieldifferent unterrichtet; zwei Schüler haben den attestierten Förderschwerpunkt emotionale-soziale Entwicklung, ein Schüler den der körperlich-motorischen Entwicklung. Diese drei Schüler arbeiten somit zielgleich. Der Klasse steht neben einem regulären Klassenraum ein Differenzierungsraum zur Verfügung. Die Hauptfächer sowie Sport, Biologie, Kunst und Musik werden in Doppelbesetzung unterricht; 20 Stunden übernimmt hier eine Sonderpädagogin. Es gib eine Arbeitsgruppe Inklusion an der Schule, die sich schon vor der Einrichtung der ersten inklusiven Klasse mit dem Thema beschäftigt hat und Fortbildungen, Informationsveranstaltungen und Austauschmöglichkeiten organisiert. Die Ganztagsschule verfügt seit Jahren über ein umfangreiches Konzept im Bereich der individuellen Förderung sowie ein mehrköpfiges Beratungsteam, das alle SuS, deren Eltern sowie das Kollegium unterstützt. Diese hier in Ansätzen aufgeführten Bedingungen schaffen eine gute Grundlage, ein Fundament, um Gemeinsames Lernen auch an einem Gymnasium umzusetzen. Die Rahmenbedingungen passen.

In der Klasse selbst werden in allen Fächern unterschiedliche Unterrichtsarrangements ein- und umgesetzt:

a) Oftmals arbeiten alle $\mathrm{SuS}$ gemeinsam im Klassenraum. Auf einen gemeinsamen Einstieg bzw. ein WarmUp (in Englisch z.B. Vokabelabfrage, Bildbeschreibung, Lied) folgt eine Erarbeitungsphase in Einzel-, Partner- und/ oder Gruppenarbeit. Die zwei Lehrkräfte haben während der Stunde die Möglichkeit, einzelne SuS zu unterstützen und abzufragen; sie können sich Ergebnisse durchlesen, anhören und anschauen.

b) Immer wieder arbeiten die SuS in Kleingruppen. Abhängig von Thema und Aufgabe werden die Gruppen eher leistungshomogen oder bewusst

5 Für ausführliche Informationen zum Themenbereich Inklusion allgemein und speziell am GSG Pulheim empfehle ich die Homepage der Schule: (http://gsg.intercoaster.de/ic/page/1963/das_gsg_auf_dem_weg_zur_inklusiven_schule.html/ 10. Januar 2015). 
gemischt zusammengestellt. Sollen sich die SuS beispielsweise eine neue Zeit (wie das Present Perfect) selbstständig erschließen, macht es wenig Sinn, die zieldifferent-geförderten SuS auf die verschiedenen Gruppen zu verteilen, da sie das grammatikalische Phänomen gar nicht brauchen bzw. nutzen. Erarbeiten sich die SuS aber eine Kurzgeschichte, können die Gruppen auch bewusst heterogen zusammengestellt werden. In dieser Klasse liegt bewusst ein großes Augenmerk auf dem Sozialen Lernen. Exkursionen in den Wald, Sportveranstaltungen, Lions-Quest-Stunden sowie Klassenratsitzungen sind fester Bestandteil des Stundenplans der Klasse.

c) Es gibt natürlich auch immer wieder Phasen, in denen die Klasse bewusst geteilt wird und die zieldifferent-geförderten SuS mit einem der beiden Lehrer oder einem leistungsstarken Mitschüler im Differenzierungsraum oder in der Bibliothek üben und wiederholen.

Gemeinsames Lernen funktioniert (natürlich) auch am Gymnasium. Gemeinsam lernen heißt (natürlich) nicht, dass alle SuS immer zur gleichen Zeit am gleichen Ort am gleichen Thema arbeiten.

Die Erfahrungen des ersten Jahres zeigen, dass vor allem die Reduktion der Inhalte in Verbindung mit einer klaren Instruktion, einer Struktur für das Vorgehen, zwei der wichtigsten Gelingensbedingungen sind, um alle SuS der Klasse mitzunehmen. Alle SuS der Klasse können am selben Oberthema arbeiten, wenn die Inhalte bzw. die Materialien für einzelne SuS reduziert und somit auf ihre Bedürfnisse abgestimmt werden. Eine Differenzierung nach Niveaustufen geht damit oftmals einher (z.B. Arbeitsblatt A, B oder C). So können beispielsweise alle SuS am Englischunterricht teilnehmen und am inhaltlichen Oberthema school arbeiten. Hierbei strukturieren und entlasten oftmals Wochenpläne die Arbeit für die SuS und ermöglichen eine individuelle Erarbeitung des Themas. Die zielgleich geförderten SuS erarbeiten sich im Kontext des Themas unter anderem eine neue Zeit (z.B. das Simple Past), die zieldifferent geförderten SuS konzentrieren sich auf die Erweiterung ihres Wortschatzes und schulen die Kompetenz Sprechen (Talking about my school). ${ }^{6}$

6 Vgl. u.a. Mendez (2012) und Lindemann (2012). Die beiden Pädagoginnen geben weitere konkrete Tipps für Umgang mit SuS im Förderschwerpunkt Lernen. 
Für die SuS mit Förderschwerpunkt geht es vor allem immer wieder darum, die Themen des gymnasialen Lehrplans so runterzubrechen und in einen sinnvollen kommunikativen Kontext einzubetten, dass sie diese als motivierend und interessant empfinden. Eine Orientierung bieten dabei die Lehrpläne der Hauptschule (NRW 2011) sowie die zahlreichen Lehrwerke der Förderschulen (u.a. Klick!; navi). Im Bereich der Kommunikativen Kompetenzen geht es somit auch um die Schulung der Teilbereiche Hörverstehen und Hör-Sehverstehen, Leseverstehen, Sprechen, Schreiben und Sprachmittlung. Auch die SuS mit Förderschwerpunkt Lernen erhalten die Möglichkeit, die gesamte Breite fremdsprachlicher Fähigkeiten und Fertigkeiten zu erwerben. Den Fokus haben wir in dieser Klasse zu Beginn auf die Wortschatzerweiterung und direkte Kommunikation gelegt. Aufbauend auf kleine Wortschatz- und Grammatikübungen werden viele Dialoge gelesen, gesprochen und gespielt: talking about my hobbies, my school or my pet, a shopping dialogue und telling my friend about my weekend - die Liste der Möglichkeiten ist lang. Visualisierungen sowie zahlreiche Sprechübungen laut und deutlich - helfen den SuS, die Aufgaben zu bewältigen.

Die SuS mit sonderpädagogischem Förderbedarf und zielgleicher Förderung erarbeiten sich beim Überthema school ebenfalls die neue Zeit eventuell wählen sie aber eine andere Methode bzw. einen anderen Zugang. Das Arbeiten mit einem Partner bzw. in Gruppen fällt den SuS im Förderschwerpunkt emotional-soziale Entwicklung oftmals noch schwer. Kooperative Lernformen können nur in einzelnen Phasen für alle SuS hilfreich sein; in vielen Stunden arbeiten die Schüler lieber für sich, unterstützt von einer Lehrkraft oder einer Schulbegleitung. Diese Öffnung hin zu einer freieren Methodenwahl bzw. einem häufigen Methodenwechsel bietet Chancen für alle SuS der Klasse. So kann jeder Schüler und jede Schülerin in einzelnen Phasen selbstständig entscheiden, wie er bzw. sie sich das neue Thema erschließt.

Der Einsatz von Lösungsbeispielen sowie Lösungen ist zentral, hilfreich und vor allem auch für die Lehrkraft entlastend. In einer Klasse, in der viele SuS zur gleichen Zeit an ganz unterschiedlichen Aufgaben arbeiten, ist eine gemeinsame Sicherungsphase kaum möglich. Das persönliche Gespräch, der Austausch mit den SuS und ein Feedback durch die Lehrkraft sind dennoch unerlässlich und vielleicht gerade in einer integrativen Lerngruppe mitentscheidend für die Motivation der Kinder. Die zieldifferent geförderten SuS 
erhalten keine Ziffernoten. Natürlich möchten sie dennoch - zu Recht - eine Rückmeldung über ihre Entwicklung, ihre Erfolge und mögliche „Baustellen“ erhalten. Die SuS sind dabei angehalten - und setzen das schon gut um - sich gegenseitig eine Rückmeldung zu Arbeitsverhalten und -ergebnis zu geben. Das persönliche Gespräch mit einzelnen SuS oder kleinen Gruppen bietet die Chance, Gemeinsamkeiten und Unterschiede transparent zu machen und das immer wieder auftretende Gefühl von Benachteiligung aus der Welt zu räumen. Jede Schülerin und jeder Schüler der Klasse wird gesehen und gehört; alle erhalten eine Rückmeldung.

Das Arbeiten auf unterschiedlichen Niveaus, das melden auch die SuS zurück, funktioniert gut. ${ }^{7}$ Von Beginn an war es für alle Beteiligten kein Problem, dass einzelne SuS keine Noten sowie zum Teil andere - leichtere Arbeitsblätter erhalten. Es führt zu keinerlei Unstimmigkeiten, dass einzelne SuS für bestimmte Arbeitsphasen in einem anderen Raum arbeiten. „Ich fühle mich deutlich wohler als in der Grundschule. Schule macht Spaß und ich lerne viel mehr", meldete beispielsweise ein zieldifferent-geförderter Schüler im letzten Lernentwicklungsgespräch zurück. Eine seiner Mitschülerinnen betont in einem Klassengespräch, wie entspannt und kooperativ die Zusammenarbeit und die Unterstützung mit den meisten Klassenkameraden funktioniert.

\section{Not bad - die ersten Ergebnisse}

Positive persönliche Eindrücke und einzelne, aus Sicht der Lehrerinnen und Lehrer wie SuS gut verlaufende Stunden sind wünschenswert und schön, reichen aber verständlicherweise nicht aus, um zu belegen, dass Gemeinsames Lernen für alle SuS funktionieren kann und die „RegelSuS“ der GL-Klasse nicht ihren Stufenkameraden in den Parallelklassen hinterher hängen oder

7 Die SuS werden in regelmäßigen Abständen befragt - schriftlich wie mündlich wie es ihnen in der Klasse geht, ob sie sich wohlfühlen, sie sich unter- oder überfordert fühlen oder ob sonst irgendwo der Schuh drückt. Die Rückmeldung der SuS ist wichtig und zentral, um durchgeführte Unterrichtsmethoden und eingesetztes Material auszuwerten und eventuell zu überdenken. Eine entsprechend angepasste Befragung findet mit allen im Klassenteam unterrichtenden Kolleginnen und Kollegen statt. Die Doppelbesetzung bietet eine gute Möglichkeit, dass eine Lehrkraft immer mal wieder beobachtet. 
die zieldifferent-geförderten SuS völlig überfordert sind. Benötigt werden mehr longitudinale Studien aus den unterschiedlichen Schulsystemen und Jahrgangsstufen, die offen- und belegen, ob Gemeinsames Lernen funktioniert, was gut gelingt und wo Baustellen bestehen. Vor allem im Kontext einer oftmals emotional aufgeladenen und öffentlich geführten Debatte aus „Fans und Gegnern der Inklusion“ können fundiert erhobene Ergebnisse helfen, die Diskussion auf den Boden der Tatsachen zurückzuholen und die Energie auf die eigentliche Arbeit in den Schulen zu lenken.

The task is to examine, carefully and analytically, how inclusive education can be effective in meeting the different needs of individual children with disabilities and special educational needs. (Lindsay 2007: 19)

Eine Möglichkeit der Erhebung von Daten können, neben Fragebögen und Unterrichtsbeobachtungen, einheitliche Kompetenzüberprüfungen in den unterschiedlichen Jahrgangsstufen, ähnlich den landesweiten Lernstandserhebungen in Jahrgangsstufe 8, sein. So haben die SuS der ersten GL-Klasse des Geschwister-Scholl-Gymnasiums und ihre Parallelklassen zum Ende der Jahrgangsstufe 5 eine einheitliche Arbeit geschrieben. Es sollte gezeigt werden, dass gute Ergebnisse nicht vom Besuch einer „Regelklasse“ abhängen und dass es in allen Parallelklassen sehr gute, gute und weniger gute SuS gibt. Die Ergebnisse bestätigen diese Annahme. Die SuS der GL-Klasse (Klasse II) gehören mit einer durchschnittlichen Punktzahl von 22 nicht nur zu den drei „besten“ Klassen, sondern erreichen die höchste durchschnittliche Punktzahl (siehe Tabelle 1).

Tabelle 1: Ergebnisse der Vergleichsarbeit „Kompetenzcheck Englisch“ Jahrgangsstufe 5 zum Ende des Schuljahres 2013/14

\begin{tabular}{|l|r|r|r|r|r|c|}
\hline Klassen & I & II & III & IV & V & VI \\
\hline durchschnittliche Punktzahl & 12 & 22 & 20 & 15 & 14 & 21 \\
\hline höchste erreichte Punktzahl & 24 & 28 & 27 & 21 & 24 & 25 \\
\hline niedrigste erreichte Punktzahl & 1 & 6 & 8 & 11 & 6 & 10 \\
\hline
\end{tabular}

Mit regelmäßigen Tests und Überprüfungen sollten ganz unterschiedliche Ziele verfolgt werden. (I) Wenn man belegen möchte, dass Inklusion, losgelöst von Ideologie und parteipolitischer Ausrichtung, funktioniert, muss belegt werden, dass zielgleich-geförderte SuS die Kompetenzerwartungen der jeweiligen Jahrgangsstufe erreichen. Konkret für diesen Kompetenzcheck 
bedeutet das, dass die Lese- und Schreibkompetenz überprüft wurde. Darüber hinaus gab es einen Grammatikteil mit Themen der Jahrgangsstufe 5 . (II) Die zieldifferent-geförderten SuS erhalten zwar keine Noten und nehmen auch nicht an den allgemeinen Kompetenzchecks teil, dennoch muss gewährleistet sein, dass auch sie einen ihren persönlichen Fähig- und Fertigkeiten entsprechenden Fortschritt erreichen. Ein mehrstufiges Verfahren wurde und wird dazu angewendet. Aufbauend auf eine Eingangsdiagnostik zu Beginn der Jahrgangsstufe 5 (Was ist da? / Was können die SuS schon?) wurden zunächst individuelle Kompetenzerwartungen aufgestellt sowie erste Materialien, wie Arbeitsblätter, Vokabellisten und Texte, zusammengestellt und teilweise selbst entworfen. Als Orientierung dienten die Oberthemen der Klasse. Eine solche erste Kompetenzerwartung kann konkret z.B. wie folgt lauten: Presenting myself and my school. Nach einem zuvor festgelegten Zeitraum, der auch den SuS transparent gemacht worden war, wurde überprüft, ob und in welcher Form die SuS das besprochene Ziel erreicht hatten. Der individuelle Lernzuwachs wurde von nun an in individuellen Förderplänen festgehalten. Welche Fähig- und Fertigkeiten beherrschen die $\mathrm{SuS}$ schon, woran müssen sie arbeiten? Für die Lehrkraft bedeutet das eine eventuelle Anpassung und Überarbeitung der Materialien sowie eine häufige Diagnose des Lernfortschritts.

(III) Diagnostik sollte ganz allgemein und für alle SuS der Klasse „zum Zwecke einer umfassenden und ganzheitlichen Förderung erfolgen “ (Liebers \& Seifert 2012: 2). Liebers \& Seifert unterscheiden zwischen summativem Zweck: Rechenschaftspflicht - und formativem - Zweck: Unterstützung Lernprozess - Assessment (ebd.: 2). Wir werden hoffentlich nie an den Punkt einer „Überprüfung“ von SuS gelangen. Die Nutzung formativer Assessment-Strategien, z.B. Lernziele transparent machen und regelmäßig überprüfen, „inwieweit der einzelne Schüler Lernfortschritte auf dem Weg zur Erreichung dieser Lernziele macht“ (Liebers \& Seifert 2012: 3), hat wahrscheinlich positivere Auswirkungen für die SuS (Liebers \& Seifert 2012: 2). Dennoch können nicht immer wieder die Vorteile von inklusiver Bildung betont werden - vor allem an Gymnasien - ohne greifbare Ergebnisse vorzulegen. Eine Verbindung aus beiden Diagnoseansätzen scheint sinnvoll wie ratsam.

Bei den hier vorgestellten Ansätzen der Überprüfung und Diagnostik müssen folgende limitierende Faktoren berücksichtigt werden: 
a) Ein einzelner Kompetenzcheck ist ein Anfang, ein erster Schritt. Dieser sollte als Ausgangspunkt für eine längsschnittartige Begleitung der GLKlassen und ihrer Parallelklassen dienen. Erst regelmäßige Überprüfungen der zu erwartenden Kompetenzen in den unterschiedlichen Jahrgangsstufen liefern valide Ergebnisse, die eine höhere Aussagekraft besitzen.

b) Der hier vorgestellte Kompetenzcheck wurde an unterschiedlichen Tagen zu unterschiedlichen Uhrzeiten in den Klassen durchgeführt. Für eine reliablere Testung sollten alle SuS am gleichen Tag in den gleichen Stunden an der Überprüfung teilnehmen.

c) Die Tests wurden nach zuvor festgelegten Kriterien korrigiert und bewertet. Dieses Vorgehen sollte auch in Zukunft gesichert sein, um eine objektive Bewertung vornehmen zu können.

d) Alle folgenden Lernstandüberprüfungen sollten sich an den in den Lehrplänen ausgeschriebenen Kompetenzerwartungen orientieren und möglichst ein breites Spektrum eben dieser abdecken. Wichtig ist in diesem Zusammenhang dann vor allem auch die Überprüfung der Kompetenz Sprechen.

e) Um eine möglichst ganzheitliche Betrachtung der Entwicklung der SuS zu ermöglichen, müssen weitere Datenerhebungsstrategien angewendet werden. Neben regelmäßigen Kompetenzchecks können beispielsweise Fragebögen an die SuS, ihre Eltern sowie die Lehrerinnen und Lehrer verteilt werden.

\section{Es gibt noch viel zu tun - ein Ausblick}

Eine große Aufgabe für die kommenden Jahre wird es sein, passende Lernmaterialien für die Schulen zu erstellen. Noch hängt es zu einem großen Teil an den unterrichtenden Lehrkräften, passendes Material zu suchen, zusammenzustellen oder zu entwerfen. So verbringen die Lehrerinnen und Lehrer viel Zeit damit, eigene Materialien zu erarbeiten. Vor allem für die höheren Jahrgangsstufen fehlt es an Lernmitteln. In diesem Zusammenhang muss es auch darum gehen, dass die Zweitspracherwerbsforschung und die Fremdsprachendidaktik noch enger zusammenarbeiten. „Eine interdisziplinäre Zusammenarbeit der Lehrenden in beiden Phasen der Lehrerausbildung ist nötig, um inklusive und fachspezifische Ausbildungsmodule zu schaffen“ (Küchler \& Roters 2014: 245). Die wissenschaftlichen Erkenntnisse müssen 
noch stärker Eingang finden in den „Klassenraum“, sodass Materialien, Methoden und Vorgehensweisen überdacht und eventuell neu angepasst werden können. Gleichzeitig sollten, den Gedanken von Preuss-Lausitz (2014) aufgegriffen, die Universitäten ihre Türen gerade für die Expertinnen und Experten aus der Praxis - die Lehrerinnen und Lehrer, die tagtäglich in inklusiven Settings arbeiten - öffnen und ihre Eindrücke und ihr Wissen nutzen, um Forschungsfragen zu entwerfen und zu realisieren.

Eine weitere große Herausforderung für den Alltag sind die SuS, die zielgleich unterrichtet werden sollen, theoretisch kognitiv auch in der Lage dazu sind, aber aufgrund ihres Förderbedarfs oftmals die Mitarbeit ablehnen und in zahlreichen Stunden gar nicht in der Lage sind, sich auf die Inhalte zu konzentrieren - völlig egal wie differenziert, aufbauend auf das Vorwissen oder aktivierend die Aufgaben auch sein mögen. Herausfordernd sind auch alle die SuS, die völlig ohne diagnostizierten Förderbedarf ihre Schullaufbahn bestreiten und dennoch auffällig in ihrem Verhalten sind. Schwierige SuS bzw. „SuS in Schwierigkeiten“ sind auch an Gymnasien keine Einzelfälle mehr. Diese SuS brauchen Aufgaben und somit Lernmittel, die sie intellektuell fordern und ihnen gleichzeitig ausreichend Raum lassen, diese individuell zu bearbeiten. Die Lehrerinnen und Lehrer, die diese SuS unterrichten sollen, brauchen Unterstützung in Form einer guten Aus- und Weiterbildung, einer unterstützenden Schulleitung und äußerer, räumlicher Differenzierungsmöglichkeiten. Konzepte von gutem Unterricht müssen in diesen inklusiven Settings neu hinterfragt werden (z.B. kooperative Lernformen).

Ohne die entsprechenden Ressourcen (u.a. ausreichend Lehrkräfte, Differenzierungsraum, Doppelbesetzung, Teamzeiten, Unterstützung durch Sonderpädagogen) und den Einsatz von motivierten Kolleginnen und Kollegen kann ein für alle Seiten gewinnbringender Unterricht nicht bzw. kaum gelingen. Gleichzeitig muss für jede Schülerin und jeden Schüler individuell geschaut werden, welche Schulform für sie oder ihn die richtige ist. Ein „Inklusionszwang“ unter dem Deckmantel der Gleichberechtigung bzw. der individuellen Förderung ist gefährlich und nutzt vor allem einer Gruppe nicht: den SuS selbst. Inklusion ist nicht realisiert, wenn einfach möglichst viele SuS eine Regelschule besuchen. Inklusion bedeutet, für jeden Menschen eine möglichst optimale Schule zu finden; für einzelne SuS kann das nach wie vor eine Förderschule sein.

Es gibt viel zu tun: Machen wir uns an die Arbeit. 


\section{Literaturverzeichnis}

Bach, Gerhard \& Timm, Johannes-Peter (2009), Englischunterricht. Grundlagen und Methoden einer handlungsorientierten Unterrichtspraxis (4. Aufl.). Tübingen: Francke.

Bongaerts, Theo (2005), Introduction: ultimate attainment and the critical period hypothesis for second language acquisition. International Review of Applied Linguistics 43: 4, 259-267.

Cook, Vivian \& Singleton, David (2014), Key Topics in Second Language Acquisition. Bristol: Multilingual Matters.

Haß, Frank (2014), Reality rules. BildungSpezial 1, 50-52.

Hinz, Klaus (1991), Schüler mit schwierigen Voraussetzungen. In: Grebing, Renate (Hrsg.) (1991), Grenzenloses Sprachenlernen. Berlin: Cornelsen, 195-204.

Klein-Landeck, Michael (2014), Inklusions-Material. Englisch 5-10. Berlin: Cornelsen.

Klemm, Klaus (2009), Sonderweg Förderschulen. Hoher Einsatz, wenig Perspektiven. Gütersloh: Bertelsmann.

KMK (Kultusministerkonferenz) (2011), Inklusive Bildung von Kindern und Jugendlichen mit Behinderungen in Schulen (Beschluss der Kultusministerkonferenz vom 20. Oktober 2011). [Online: http://www.kmk.org/ fileadmin/veroeffentlichungen_beschluesse/2011/2011_10_20-InklusiveBildung.pdf 23.01.2015].

Köpfer, Andreas (2014), Kernkategorien einer inklusiven Englischdidaktik. In: Bartosch, Roman \& Rohde, Andreas (Hrsg.) (2014), Im Dialog der Disziplinen: Englischdidaktik - Förderpädagogik - Inklusion. Trier: Wissenschaftlicher Verlag, 157-166.

Küchler, Uwe \& Roters, Bianca (2014), Embracing Everyone: Inklusiver Fremdsprachenunterricht. In: Amrhein, Bettina \& Dziak-Mahler, Myrle (Hrsg.) (2014), Fachdidaktik inklusiv. Auf der Suche nach didaktischen Leitlinien für den Umgang mit Vielfalt in der Schule. Münster \& New York: Waxmann, 233-248.

Lazda-Cazers, Rasma \& Thorson, Helga (2008), Teaching Foreign Languages to Students with Disabilities: Initiatives to Educate Faculty. In: Berberi, Tammy; Hamilton, Elizabeth C. \& Sutherland, Ian M. (Hrsg.) (2008), Worlds Apart? Disability and Foreign Language Learning. New Haven \& London: Yale University Press, 107-133. 
Liebers, Katrin \& Seifert, Christin (2012), Assessmentkonzepte für die inklusive Schule - eine Bestandsaufnahme. Zeitschrift für Inklusion 3. [Online: http://www.inklusion-online.net/index.php/inklusion-online/ article/view/44/44].

Lindemann, Beate (2012), So könnte es gehen! Inklusiver Englischunterricht. Praxis Fremdsprachenunterricht Englisch 1, 15-16.

Lindsay, Geoff (2007), Educational psychology and the effectiveness of inclusive education / mainstreaming. British Journal of Educational Psychology 77, 1-24.

Mendez, Carmen (2012), Inklusion im Fremdsprachenunterricht. Herausforderung und Chance. Praxis Fremdsprachenunterricht Basisheft 1, 5-8.

Millrood, Radislav (2002), Teaching heterogeneous classes. ELT Journal 56: 2, 128-135.

Ministerium für Schule und Weiterbildung des Landes Nordrhein-Westfalen (2007), Kernlehrplan für das Gymnasium - Sekundarstufe I (G8) in Nordrhein-Westfalen. Englisch. Düsseldorf.

Ministerium für Schule und Weiterbildung des Landes Nordrhein-Westfalen (2011), Kernlehrplan für die Hauptschule in Nordrhein-Westfalen. Englisch. Düsseldorf.

Myklebust, Jon Olav (2006), Class placement and competence attainment among students with special educational needs. British Journal of Special Education 33: 2, 76-81.

Preuss-Lausitz, Ulf (2014), Wissenschaftliche Begleitungen der Wege zur inklusiven Schulentwicklung in den Bundesländern. Versuch einer Übersicht. [Online: http://www.unesco.de/fileadmin/medien/Dokumente/Bildung/ Wiss Begleitung Inklusion end 2 .pdf 14.01.2015].

Rohde, Andreas \& Schick, Kim (2012), Englisch für alle: Wie Schüler individuell gefördert werden. At Work. Das Englisch-Magazin von Diesterweg 21, 3-4.

Singleton, David (2005), The Critical Period Hypothesis: a coat of many colours. IRAL 43: 4, 269-285.

Sturm, Tanja (2013), Lehrbuch Heterogenität in der Schule. München / Basel: Reinhardt Verlag, UTB.

UN (2008), Die UN-Behindertenrechtskonvention, Übereinkommen über die Rechte von Menschen mit Behinderungen. [Online: https://www. 
behindertenbeauftragter.de/SharedDocs/Publikationen/DE/Broschuere_ UNKonvention_KK.pdf?_blob=publicationFile 23.01.2015].

Weskamp, Ralf (2007), Fachdidaktik: Grundlagen \& Konzepte. Anglistik. Amerikanistik. Berlin: Cornelsen. 
Christiane M. Bongartz and Andreas Rohde - 978-3-653-97188-0

Downloaded from PubFactory at 01/11/2019 10:41:16AM

via free access 


\section{Ulla Schäfer \\ Inklusives Lehren und Lernen im Englischunterricht}

Inclusive English teaching also aims to adopt the characteristic features of what is generally referred to as "good and effective English teaching”. Based on Meyer's criteria for good teaching, a transparent lesson structure for the inclusive English classroom is proposed which introduces picture cards and chunks that serve as scaffolds and guide the learners through the lesson structure. This structure features welcome and closing routines as well as clearly defined and communicated objectives. It will also be shown that general principles of modern second language teaching have their role in inclusive teaching, such as "English only", corrective feedback rather than direct error correction and a rich inventory of methods and approaches. In addition, the author suggests a help-desk that can be arranged and used by all students irrespective of any specific individual needs.

Seit der Ratifizierung der UN-Konvention 2009 haben alle Schülerinnen und Schüler (SuS) in Deutschland ein Recht auf Unterricht in einer Regelschule, was zu Veränderungen in unseren sogenannten Regelklassen führt. Auch wenn es die homogene Klasse nie gegeben hat und Heterogenität immer Alltag in allen Schulen war und ist, rufen die neuen Herausforderungen nach adäquaten Ideen. Ein Patentrezept gibt es nicht, da sehr unterschiedliche Bedürfnisse immer wieder individuelle Konzepte erforderlich machen.

Viele Lehrende sind verunsichert, weil sie sich auf diese Situation nicht ausreichend vorbereitet fühlen. Fort- und Weiterbildung hat es bisher nicht ausreichend überall gegeben. Regelschullehrende sind aber bisher nicht im Bereich Förderschwerpunkte ausgebildet. So soll es ein wenig Mut machen, dass der vor einigen Jahren in der Grundschule eingeführte Englischunterricht, wenn er an den Prinzipien moderner Fremdsprachendidaktik und Erkenntnissen aus der Spracherwerbsforschung ausgerichtet ist, gute Voraussetzungen für „Inclusivity“ in Bezug auf verschiedene Förderbedarfe bietet.

Inklusiver Englischunterricht ist somit kein völlig anderer als Unterricht in Regelklassen, und seine Methoden und Prinzipien sind nicht gänzlich verschieden. Es sind jedoch methodische Modifikationen erforderlich. So 
sind bspw. Lernspiele nicht neu; wie aber können sie so organisiert werden, dass sich auch Schüler mit bestimmten Beeinträchtigungen erfolgreich daran beteiligen können? Es gibt nicht das didaktische Modell für den inklusiven Englischunterricht, jedoch „förderliche Grundhaltungen und Einstellungen der Pädagogen und Schüler, hilfreiche Prinzipien und Methoden und bewährte Unterrichtsformen, welche eine inklusive Lernkultur begünstigen “ (Klein-Landeck 2014: 5).

Im Folgenden soll anhand von Kriterien für guten Englischunterricht verdeutlicht werden, wie inklusiver Englischunterricht gelingen kann. $\mathrm{Zu}$ bedenken ist vorab, dass Veränderungen in Bezug auf Räume, Personal, Lehrmaterialien und Lehrwerke unabdingbar sind. Außerdem sind Fortund Weiterbildungen für Lehrende unbedingt notwendig, damit diese den Bedürfnissen von SuS aus unterschiedlichen Förderschwerpunkten Genüge tun können.

\section{Kriterien guten Unterrichts}

An Meyers Kriterien für guten Unterricht ausgerichtet (Meyer 2004), sollen Kriterien guten Englischunterrichts beschrieben werden. Er benennt zehn Kriterien für guten Unterricht, die im Folgenden jedoch nicht alle gleichermaßen ausführlich bearbeitet werden können.

\section{Klare Strukturen des Unterrichts}

Englischunterricht braucht ebenso wie jeder andere Unterricht klare Strukturen. Transparenz in Bezug auf das Unterrichtsgeschehen und ritualisierte Stundenabläufe geben SuS Sicherheit. Das trifft umso mehr für SuS im Förderschwerpunkt Lernen zu.

Der im Jahre 2003 in NRW eingeführte Englischunterricht an Grundschulen war von Anfang an stark ritualisiert, um jungen Lernern das $\mathrm{Zu}$ rechtfinden in einer Unterrichtsstunde, die weitgehend in englischer Sprache abgehalten wurde, zu erleichtern.

\section{Transparenz}

Die Verlaufstransparenz zu Beginn jeder Stunde sorgt dafür, dass SuS wissen, was auf sie zukommt und wo sie sich innerhalb des Unterrichtsgeschehens befinden. 
Ein grober Überblick zu Beginn einer Lerneinheit erfüllt zwei wichtige Funktionen: Zum einen bereitet er die SuS auf die bevorstehenden Inhalte vor, was später zu einer besseren Wiedererkennungsleistung führt. Zweitens fungieren Überblick und Vorstellung der Struktur der Lerneinheit als externale Einspeicherungshilfe im Sinne einer Organisation der Lerninhalte. (Brand \& Markowitsch 2009: 81)

Für die Verlaufstransparenz kann der Lehrende z.B. Symbolkarten verwenden, die in entsprechender Reihenfolge visualisiert werden. Diese verdeutlichen die einzelnen Elemente einer Stunde und können bereits ein erster Sprechanlass sein. SuS äußern sich ihrem Sprachstand entsprechend zu den Karten und den dadurch repräsentierten Unterrichtselementen. Dabei sprechen sie anfangs Deutsch, bedienen sich nach und nach ihrer Lernersprache, bis es ihnen gelingt, korrekte chunks zu verwenden.

Wenn eine Situation für SuS bedeutungsvoll ist, sie wirklich sprechen wollen, ihnen dazu aber die korrekten sprachlichen Mittel fehlen, müssen sie nach und nach lernen, mit entsprechenden Strategien ihr Anliegen vorzutragen. Diese Fähigkeit muss geübt werden und sollte neben dem Einüben von chunks eine wichtige Aufgabe des Englischunterrichts sein. Es handelt sich um eine Kompetenz, die in alltäglichen Sprachhandlungssituationen einsetzbar ist.

Des weiteren verwenden SuS im Rahmen der Verlaufstransparenz einen entsprechenden chunk, der so in jeder Stunde geübt wird. Als Beispiel soll die Struktur "We are going to...“ betrachtet werden. Das Near Futurel Going to Future ist wichtig, da es im authentischen Sprachgebrauch häufig verwendet wird. Im Wissen, dass das Gehirn am besten lernt, wenn es mit bedeutungsvollen Inhalten konfrontiert wird, sollten Lehrende darauf achten, dass die angebotenen Situationen und Sprachemittel „meaningful“ sind. Es muss einen Grund geben, die Fremdsprache zu erlernen und zu nutzen. Die Motivation für den Gebrauch der Fremdsprache entsteht in authentischen Situationen (Nunan 2004).

Wie die Verlaufstransparenz ist auch die Zieltransparenz ein wichtiges Element im Lernprozess. Sie hilft SuS bewusst mit der möglichen Kompetenzerweiterung umzugehen. Deutlich wird das spätestens bei der Reflexion am Ende einer Stunde. Idealerweise wird SuS verdeutlicht, dass ein Ziel auf unterschiedlichen Wegen und auch auf unterschiedlichen Leistungsebenen $\mathrm{zu}$ erreichen ist: 
Today you can learn new words by... Today you can learn new words and the new sentence...

Bei der Zieltransparenz kann, genau wie bei Arbeitsanweisungen, die Sprachmittlung Verwendung finden. Lehrpläne fordern diese Technik als sogenannte fünfte Fertigkeit, die in die Leistungsbewertung einfließen muss. Eine Technik, die bewertet wird, muss erlernt und geübt werden. Dafür braucht es authentische Gelegenheiten innerhalb des Unterrichts.

\section{Anfangsrituale}

Rituale sind wichtige Voraussetzungen für sinnvolles Lernen. So sind Anfangs- und Endrituale, sowie ritualisierte Übungsformate feste Bestandteile des Englischunterrichts an der Grundschule, wenn dieser den Kriterien moderner Fremdsprachendidaktik entspricht. Stunden beginnen z.B. mit Begrüßungsritualen/Warming-ups in Form von Songs, Rhymes, Chants in englischer Sprache. Ein solches Anfangsritual hilft SuS, sich entsprechend auf den Englischunterricht einzustellen. Wie ein Anfangsritual aussieht, sollte mit der jeweiligen Lerngruppe gemeinsam herausgefunden werden. Sehr beliebt sind bei vielen Lehrenden Begrüßungslieder, die a capella oder unterstützt durch eine CD, begleitet von Mimik und Gestik, gesungen werden. Reime werden gemeinsam gesprochen. So wird die Aussprache geübt: "Songs, rhymes and chants are particularly useful for practising pronunciation. This includes individual sounds and sounds in connected speech but, more importantly, features relating to stress, rhythm and intonation" (Brewster, Ellis \& Girard 2003: 178).

\section{Phasenwechsel / Rhythmisierung}

Eine angemessene Rhythmisierung, ein adäquate Phasenwechsel, der in der Literatur immer wieder angemahnt wird (Meyer 2004), haben einen besonderem Stellenwert, vor allem für SuS mit Lernschwierigkeiten. Allerdings darf im Rahmen von Phasenwechseln kein neuer Schwerpunkt entstehen. Gehen wir davon aus, dass, bildlich gesprochen, Lerner um einen Tisch herum sitzen, auf dem sich in der Mitte der Lernstoff befindet, der betrachtet wird. Bereits nach kurzer Zeit, ca. 10-15 Minuten, wird dieser Ausblick langweilig und der Lernende beginnt abzuschweifen. Nun wäre es aber fatal, den Lernstoff zu wechseln. Vielmehr wird dem Lerner ein neuer Platz 
am Tisch angeboten. Aus der neuen Perspektive heraus betrachtet wird der Lernstoff wieder interessant. Beim Phasenwechsel darf es nicht um einen Wechsel des Lernstoffs gehen, sondern um einen neuen Blickwinkel auf denselben. Die Sache an sich als Möglichkeit zur Kompetenzerweiterung bleibt erhalten (Meyer 2004).

\section{Reflexion / Endrituale}

Das Reflektieren und Steuern eines Lernprozesses stellt für Kinder eine große Herausforderung dar, die nur mit der Unterstützung und Hilfestellung der Lehrenden bewältigt werden kann. Daher sollte die Reflexion des eigenen sprachlichen Lernens ein Unterrichtsritual sein, das - wie in anderen kommunikativen Situationen auch - sprachstandsgemäß erfolgen sollte. Sinnvolles Lernen und die Fähigkeit, sich selbst einzuschätzen, benötigen den Weg über die Reflexion des eigenen Lernprozesses. So können sich die ersten Überlegungen im Rahmen einer langsamen Hinführung darauf beziehen, was schwer oder leicht war: „Was it easy? Was it difficult? Were there problems? "Später wird darüber nachgedacht, was am Ende einer bestimmten Unterrichtsphase gelernt oder geübt wurde: „What have you learned? “, „What have you found out? “ Selbsteinschätzung ist aber nicht nur ein wichtiger Schritt im Lernprozess, sondern: Unabhängigkeit, Kreativität und Selbstvertrauen werden gefördert, wenn Selbstkritik und Selbstbeurteilung von grundlegender Bedeutung sind (Rogers 1991).

\section{Hoher Anteil echter Lernzeit}

Englischunterricht sollte weitgehend einsprachig sein, um SuS mit dem notwendigen Input zu versorgen. Input heißt im Sinne des Spracherwerbs nach Krashen, dass SuS mit einem reichhaltigen Sprachangebot versorgt werden, was einerseits für sie verstehbar, andererseits ein wenig fordernd ist (Krashen 1981). Jedoch gibt es auch Situationen, die die Muttersprache notwendig machen.

Die Lehrpläne berücksichtigen jedoch auch, dass bereits die Organisation des fremdsprachlichen Unterrichts an Grenzen stößt, die auch den Einsatz der Muttersprache sinnvoll machen. Mit Rücksicht auf die Kinder muss für die folgenden Situationen ein Freiraum existieren: Wenn etwas Unverstandenes das einzelne Kind belastet, wenn englischsprachige Arbeitsanweisungen nicht 
verstanden werden, wenn es zu Missverständnissen in der Unterrichtsorganisation kommt, wenn komplexe Erklärungen schlicht zu viel der kostbaren Unterrichtszeit in Anspruch nehmen, wenn über kulturelle Andersartigkeit, über „das Fremde“ reflektiert werden soll, dann, darin sind sich die Lehrpläne weitgehend einig, kann die Muttersprache eingesetzt werden. In allen diesen Fällen handelt es sich im weitesten Sinne um Unterrichtsorganisation, wobei mit Blick auf die Muttersprache gelten sollte: „As little as possible, no more than necessary“ (Butzkamm \& Schmid-Schönbein 2008: 6-8).

Der Einsatz der Muttersprache sollte jedoch die Ausnahme bleiben, denn, um kommunikative Kompetenzen in bedeutungsvollen, authentischen und konkreten Sprachverwendungssituationen entwickeln zu können, ist die Fremdsprache als Unterrichtssprache vonnöten. Mit entsprechenden sprachlichen Hilfen wird während der Prozesse, z.B. bei Spielen, bei gap-filling-activities, beim storytelling u.ä. in der Fremdsprache kommuniziert, denn nur in solchen konkreten Sprachverwendungssituationen kann Spracherwerb stattfinden. SuS brauchen also immer wieder sinnvolle handlungsorientierte Anlässe - Tasks - Aufgaben, die einen sprachlichen Output notwendig machen. So werden z.B. in Spielsituationen bestimmte Äußerungen notwendig, um am Spiel teilnehmen zu können. Ein Quartettspiel (Happy Family) macht erforderlich, bestimmte Informationen zu erfragen. Ohne die Verwendung von Sprache kann man am Spiel nicht teilnehmen und schon gar nicht erfolgreich sein. Da Kinder gerne spielen und auch gerne gewinnen, handelt es sich also bei einem Spiel um eine handlungsorientierte Sprachverwendungssituation. An dieser Stelle sei nochmals darauf hingewiesen, dass „Handlungsorientierung “ in Bezug auf das Fremdsprachenlernen eine Sprachhandlung meint und nicht mit Handeln im Sinne von Kinästhetik verwechselt werden darf (Rohde 2012).

Neben fachspezifischen Lernformen sind die Prinzipien moderner Fremdsprachendidaktik von großer Bedeutung, als da wären:

Prinzip

$>$ des spielbetonten Lernens

$>$ der Einsprachigkeit

$>$ der vorwiegenden Mündlichkeit

$>$ der kommunikativen Progression 
$>$ der methodischen Vielfalt

$>$ des Einbezugs fächerübergreifender Aspekte

$>$ der Zielorientierung

$>$ der Lernerorientierung

$>$ der ständigen Vergewisserung über den Lernstand

$>$ der Transparenz der Leistungsermittlung und Leistungsbeurteilung

$>$ der erhöhten Fehlertoleranz (Error/Mistake)

Da Sprache nur individuell vom Lerner erworben werden kann, muss es die Aufgabe von Lehrenden sein, die günstigsten Bedingungen für Spracherwerb zu schaffen. Spracherwerb, der sich in Stufen vollzieht und nicht beeinflusst werden kann, in dem Sinne, dass Unterricht dazu führt, Stufen zu erreichen oder sogar zu überspringen, ist vielleicht über ein Bild gut zu verdeutlichen: Ein Krabbelkind, das lernen soll, eine Treppe herauf zu krabbeln, braucht den Raum zum Üben und Eltern, die im Falle eines „Fehltritts“ da sind, das Kind auffangen und vor einem Sturz bewahren. Genau das ist unsere Aufgabe als Lehrende im Fremdsprachenunterricht. Der Lerner macht sich auf, von einer Spracherwerbsstufe zur anderen zu kommen, versucht, scheitert, nutzt Lernersprache. Das alles im Vertrauen darauf, dass der Lehrende da ist, um helfend zur Seite zu stehen. Und genauso wenig, wie ein Krabbelkind durch reines Zusehen lernen wird, eine Treppe heraufzukrabbeln, so wenig werden SuS durch reines Nachplappern oder ausschließliches Zuhören zum Erfolg kommen.

\section{Lernförderliches Klima}

Die Freude und das Interesse am Sprachenlernen soll gefördert werden. Dazu ist es erforderlich, mit der silent period und mit Sprechhemmungen umzugehen. SuS, die sich noch nicht trauen, vor der Klasse zu sprechen, ihre Lernersprache zu nutzen, sollten zum gemeinsamem reproduktiven Sprechen durch den Einsatz von Liedern, Reimen, Spielen und u.ä. ermuntert werden. Gerade SuS aus den Förderschwerpunkten Sprache, Lernen und Emotionale/Soziale Entwicklung sollte methodisch in dieser Hinsicht immer wieder entgegengekommen werden. So kann z.B. das leise Murmeln des neuen Wortschatzes, bevor dieser chorisch laut nachgesprochen wird, oder aber in Phasen der Stillarbeit, sehr hilfreich sein. Es kann früh auch für die Hausaufgaben angebahnt werden. 
Außerdem brauchen SuS im Förderschwerpunkt Lernen oder Sprache zusätzliche Speicherkriterien:

- Strukturierung von Wörtern, z.B. durch Klatschen oder Stampfen.

- Bewegungsspiele, die z.B. eine Entscheidung zwischen kurzen und langen Wörtern fordern: Stand on your right foot when you hear a short word. Stand on...

Wichtig ist der angemessene Umgang mit Fehlern, die wichtige Schritte im Prozess des Spracherwerbs sind. Eine Differenzierung zwischen error und mistake ist wichtig, damit Fehler sich als produktive Schritte erweisen können.

Als errors werden Fehler bezeichnet, die „entwicklungsbedingt“ sind. [...] Mistakes hingegen sind (Flüchtigkeits-) Fehler, die sich meist aufgrund von mangelnder Aufmerksamkeit ereignen. [...] Nur im letzteren Fall ist das „Ahnden eines Fehlers“ angebracht. (Rohde 2011: 37f.)

\section{Inhaltliche Klarheit}

Inhaltliche Klarheit bedeutet sinnvolle und überschaubar aufgebaute Unterrichtseinheiten zu komplexen Lernsituationen sowie verständliche Aufgabenstellungen. Transparenz (Verlaufs- und Zieltransparenz, s.o.) muss vorhanden sein. Dabei sind Visualisierungen unabdingbar.

Seit es den Englischunterricht an Grund- und Förderschulen gibt, wird das Thema Schriftsprache kontrovers diskutiert. Forschungsergebnisse liegen z.B. von Diehr \& Rymarczyk (2010) sowie von Duscha (2007) vor. Für SuS mit auditiver Verarbeitungs- und Wahrnehmungs-Störung (AVWS), deren Problematik schwer zu erkennen und oft nicht diagnostiziert ist, stellt das Schriftbild eine große Hilfe dar. Bei solchen SuS liegt neben anderen Schwierigkeiten eine verringerte auditive Merkspanne vor, da das Kurzzeitgedächtnis für Gehörtes zu schwach ist.

Das Schriftbild kann für viele Lernende in Hinblick auf inhaltliche Klarheit von großer Wichtigkeit sein. Es sollte aber selbstverständlich immer erst eingeführt und zur Verfügung gestellt wird, wenn die Aussprache geklärt und geübt ist. Auf das Thema Lesen in der Fremdsprache ausführlich einzugehen, ist hier nicht der Ort. Wichtig ist, dass Lesen ermöglicht wird und das Schriftbild zur Verfügung steht.

So hat sich z.B. bei Grund- und Förderschülern die Blitzlesemethode als sehr hilfreich erwiesen, da sie Lernenden hilft, durch das Abspeichern ganzer 
Wörter oder Strukturen, sich diese besser zu merken oder wiederzuerkennen (Frisch 2013). In der Zusammenfassung ihrer Studie schreibt Frisch: „Es ist wichtiger, DASS gelesen wird, als WIE das Lesen gelehrt wird, solange die Lesemotivation erhalten bleibt. “ (Frisch 2013: 215). Sie stellt fest, dass Schülerinnen und Schüler, denen Schrift in einem höheren Ausmaß zur Verfügung stand als anderen, bessere Lernergebnisse zeigten (ebd.). Weitere Unterstützung für inhaltliche Klarheit entsteht durch die funktionale Nutzung der Sprachmittlung/Mediation, die in den Kernlehrplänen NRW gefordert wird.

\section{Sinnstiftendes Kommunizieren}

Häufig vorkommende und vertraute Gesprächssituationen im Unterricht sollten sehr regelmäßig für sinnvolle Kommunikation genutzt werden. Dazu muss SuS einerseits Sprache zur Verfügung gestellt, ihnen aber andererseits Möglichkeiten zum kreativem und experimentierenden Umgang mit der englischen Sprache eröffnet werden. Hier bietet sich immer wieder der classroom discourse an, im Rahmen dessen geeignete Redemittel kontinuierlich ausgebaut und visualisiert werden. Nur in für sie sinnvollen Situationen werden SuS elementare sprachliche Mittel erproben und festigen.

Wichtig ist, Kommunikation als Leitziel ernst zu nehmen. Es geht um message before accuracy (MSW NRW 2008), also darum, SuS erfahren zu lassen, dass sie sich in dieser Sprache unter Einsatz all ihrer kommunikativen Fertigkeiten verständigen können. Nicht das korrekte Nachsprechen vorgegebener Sätze ist das ausschließliche Ziel!

\section{Methodenvielfalt}

Die unterschiedlichen Bedürfnisse von Lernenden bedürfen verschiedener Sozial- und Arbeitsformen sowie der Elemente des kooperativen Lernens (z.B. Jig Saw, Double Circle, Chat Point, Guards). Letztere können zu authentischen Sprachhandlungssituationen führen, für die aber entsprechende Redemittel erarbeitet und angeboten werden müssen.

Öffnung von Unterricht kann auch in Form von Stationenlernen, MiniProjekten und an außerschulischen Lernorten geschehen. Hier müssen bestimmte Regeln eingehalten werden, um effektive Kompetenzerweiterungen zu gewährleisten. Stationen, die ohne Differenzierung oder individuellen Zugang angeboten werden, führen zu einer ausschließlich temporären 
Differenzierung und sind von Individualisierung weit entfernt. Im Sinne der Letzteren muss jede Station differenzierte Lösungsmöglichkeiten zulassen (Bäumer et al. 2010).

\section{Individuelles Fördern}

Um individuell fördern zu können, müssen Lehrende in der Lage sein, unterschiedliche sprachliche Entwicklungen in ihrer Bandbreite diagnostizieren zu können und diese zu akzeptieren (Spracherwerbsstufen). Hier sind neben Chunk Learning immer wieder freie Sprechanlässe von großer Bedeutung. Eine gegenseitige Unterstützung durch leistungsheterogene Paare und Gruppen kann eingeplant werden; allerdings muss der Schwerpunkt auf den Aufgabenformaten liegen. Neben reproduktiven und produktiven Aufgabenstellungen in den drei Anforderungsbereichen sollte darauf geachtet werden, dass die Aufgaben nicht ausschließlich nur eine Lösung zulassen. Die Öffnung von Aufgaben ist ein großer Schritt in Richtung auf Individualisierung. Aufgaben müssen so angeboten werden, dass SuS einen individuellen Zugang finden und mit den entsprechenden Hilfen zu einer Lösung kommen können. Hilfen sind also wichtig, um individuelle Vorgehensweisen zu unterstützen. Aufgabenformate, die individuell unter Nutzung von entsprechenden Hilfen gelöst werden können, sind lernerorientiert, im Gegensatz zu differenzierten Arbeitsblättern, bei denen die Differenzierung immer vom Lehrenden ausgeht (Böttger 2005).

\section{Intelligentes Üben}

Das Gelernte muss abwechslungsreich geübt und wiederholt werden (z.B. durch Spiele, Geschichten, Rollenspiele, Dialoge, Interviews, Rätsel und weitere Übungsrituale). Der Einsatz zu vieler verschiedener Übungsformate kann allerdings für schwache Lerner eine zusätzliche Schwierigkeit darstellen, da sie sich immer wieder auf neue Regeln konzentrieren müssen und so ihre Aufmerksamkeit nicht voll und ganz dem zu übenden Lehrstoff widmen können.

Unterrichtsmaterialien sollten stets kritisch dahingehend gesichtet und gezielt auswählt werden, ob sie tatsächlich das üben, was geübt werden muss und vor allen Dingen, inwieweit sie verschiedene Zugänge anbieten (Breuer et al. 2006). 


\section{Transparente Leistungserwartungen}

Das sehr komplexe Thema der Leistungsmessung soll hier nur durch Stichworte Erwähnung finden. Leistungsbeobachtungen sollten in geeigneten Dokumenten festgehalten werden (z.B. Beobachtungsbögen, Lerntagebücher und Portfolios der Kinder). Die Erfahrung zeigt, dass im Sinne der Transparenz Kriterien für die Bewertung von Leistungen zu Beginn eines Themas / einer Einheit / eines Halbjahres für SuS klar bereit gestellt werden sollten, weil so das Reflektieren des Lernprozesses erleichtert wird. In Bezug auf Bewertungskriterien führt erfahrungsgemäß Transparenz für Eltern zu einer besseren Zusammenarbeit zwischen Schule und Elternhaus und sollte auch eine Selbstverständlichkeit sein.

\section{Vorbereitete Lernumgebung}

Um eine adäquate Lernumgebung zu schaffen, bietet es sich an, geeignete Materialien/Medien bereitzustellen, Arbeitsergebnisse sichtbar auszustellen, Poster oder Plakate begleitend zum Thema als Sprechanlass aufzuhängen. Hilfen zum classroom discourse müssen gut sichtbar bereit stehen. Verschiedene Wörterbücher, Wortsammlungen, Strukturhilfen und weitere Materialien können z.B. auf einem „Help Desk“ (siehe auch Little Early Bird und Early Bird, Finken Verlag, s.o.) zur Verfügung stehen. Dabei ist ganz wichtig, dass die Hilfsmateralien vor den Arbeitsphasen thematisiert werden und möglichst alle Materialien an einem Ort bereit liegen. Das gilt auch für sehr leistungsstarke SuS. Nur so kann verhindert werden, dass durch die Inanspruchnahme des Hilfetisches Stigmatisierungen entstehen.

\section{Fazit}

Gut strukturierter Englischunterricht, der sich an den Kriterien guten Unterrichts sowie den Prinzipien moderner Fremdsprachendidaktik ausrichtet, bietet Chancen, auf individuelle Bedürfnisse von SuS in ganz unterschiedlichen Lernsituationen einzugehen. Lehrende müssen nur den Mut haben, sich vom „Page turning teaching“, d.h. reinem Lehrwerkunterricht zu lösen. Dazu benötigen sie Fort- und Weiterbildungen und äußere Bedingungen, die einen solchen Unterricht erleichtern. 


\section{Literaturverzeichnis}

Bäumer, Janina; Bluhm, Janina \& Scholz, Rebekka (2010), Early Bird. Oberursel: Finken Verlag.

Brand, Matthias \& Markowitsch, Hans Joachim (2009), Lernen und Gedächtnis aus neurowissenschaftlicher Perspektive - Konsequenzen für die Gestaltung des Schulunterrichts. In: Herrmann, Ulrich (Hrsg.) (2009), Neurodidaktik. Weinheim: Beltz, 69-85.

Breuer, Lis; Debrais, Peter \& Schäfer, Ulla (2006), Modul 7 für die Moderatorenausbildung in NRW zum Thema „Englisch an Förderschulen mit Förderschwerpunkt Lernen".

Böttger, Heiner (2005). Englisch lernen in der Grundschule. Bad Heilbrunn: Klinkhardt.

Brewster, Jean; Ellis, Gail \& Girard, Dennis (2003), The Primary English Teacher's Guide. London: Pearson Educated Limited.

Butzkamm, Wolfgang \& Schmid-Schönbein, Gisela (2008), Funktionale Fremdsprachigkeit - Zur Rolle der Sprachen im Englischunterricht. Grundschulmagazin 5, 6-8.

Diehr, Bärbel \& Rymarczyk, Jutta (Hrsg.) (2010), Forschung zum Schrifterwerb in der Fremdsprache bei Grundschülern. Frankfurt: Peter Lang.

Duscha, Michael (2008), Schrifteinsatz im Englischunterricht. In: Christiani, Reinhold \& Cwik, Gabriele (Hrsg.) (2008), Englisch unterrichten in Klasse 1 und 2. Berlin: Cornelsen Scriptor, 68-77.

Frisch, Stefanie (2013), Lesen im Englischunterricht der GrundschuleEine Vergleichsstudie zur Wirksamkeit zweier Lehrverfahren. Tübingen: Gunter Narr.

Klein-Landeck, Michael (2014), Inklusionsmaterial: Englisch Klasse 5-10. Berlin: Cornelsen.

Krashen, Stephen D. (1981), Second Language Acquisition and Second Language Learning. Oxford: Pergamon.

Meyer, Hilbert (2004), Was ist guter Unterricht? Berlin: Cornelsen Scriptor. MSW NRW (Ministerium für Schule und Weiterbildung des Landes Nordrhein-Westfalen) (2008), Kernlehrplan Englisch. [Online: http:// www.schulentwicklung.nrw.de/lehrplaene/upload/klp_gs/GS_LP E.pdf 31.05.2015]. 
Nunan, David (2004), Task-Based Language Teaching. Cambridge: Cambridge University Press.

Rogers, Carl R. (1991), Lernen in Freiheit, München: Kösel Verlag.

Rohde, Andreas (2011), Mein Freund, der Fehler - 'he, she, it - muss das "s" schon mit'? Grundschulmagazin Englisch 5, 37-38.

Rohde, Andreas (2012), Handlungsorientierter Englischunterricht: Nur Basteln und Singen? Grundschulmagazin Englisch 6/2012, 37-38. 
Christiane M. Bongartz and Andreas Rohde - 978-3-653-97188-0

Downloaded from PubFactory at 01/11/2019 10:41:16AM

via free access 


\section{Daniela Elsner \\ Inklusion von Herkunftssprachen - Mehrsprachigkeit als Herausforderung und Chance}

This article draws attention to a very specific group of learners: children who grow up with two or more languages, who are used to mixing languages or switch between their accompanying worlds back and forth - and who are aware of their cultural and social differences from birth on. However, multilingualism does not always receive recognition. It is in fact very often associated with discrimination and stereotyping. Thus, biographies of multilingual learners are very heterogeneous. Yet at the same time their diverse backgrounds have a significant influence on their learning outcomes in school, no matter the subject. It is one of the major aims of our schools today (especially in terms of inclusive education) to deal with these various linguistic and cultural experiences of multilingual learners. The present article focuses on the question of how, to what extent and under which conditions these pupils can actually draw upon their previous knowledge when learning additional languages such as English in school. Even though it has to be concluded that there is no simple answer to this question, some implications for successful inclusive education of family languages can be pointed out.

Legal Alien

Bi-lingual, Bi-cultural, able to slip from "How's life?"

to "Me'stan volviendo loca,"

(...)

American but hyphenated, viewed by Anglos as perhaps exotic, perhaps inferior, definitely different, viewed by Mexicans as alien, (...)

an American to Mexicans

a Mexican to Americans

(Ausschnitt aus dem Gedicht Legal Alien von Pat Mora 1985) 


\section{Einleitung - was es heißt mehrsprachig zu sein}

In ihrem Gedicht Legal Alien gibt Pat Mora ihrer Leserschaft einen unmittelbaren Einblick in die Gedanken-, Gefühls- und Lebenswelt eines Menschen, dessen Identitätsentwicklung durch die migrationsbedingte Verschmelzung von zwei Sprachen und Kulturen geprägt wurde. Die Ausführungen der IchErzählerin machen deutlich, welche Vor- und Nachteile Mehrsprachigkeit und Mehrkulturaltiät offensichtlich mit sich bringen - so ist die Erzählerin zwar in der Lage in zwei Sprachen und damit auch in zwei Welten zu agieren - eine Kompetenz, um die sie von anderen durchaus beneidet wird gleichzeitig fühlt sie sich jedoch keiner Welt wirklich zugehörig. „You are not like me" - ist das, was sie sowohl in ihrem mexikanischen Umfeld erfährt, als auch im amerikanischen Sprach- und Kulturkontext realisiert.

Die 1942 in El Paso, Texas geborene Lateinamerikanerin Pat Mora befasst sich in ihren zahlreichen Gedichten und Geschichten vornehmlich mit den Lebensgeschichten, Wahrnehmungen und Erlebnissen der Mexican Americans und der US Latinos in der amerikanischen Gesellschaft, dabei haben Moras eigene transkulturelle Entwicklung und ihre translingualen Erfahrungen ihren persönlichen Angaben nach ihre Schriften stark beeinflusst. In Amerika gilt sie als Living Voice der mehr als drei Milliarden Menschen, die dort von Geburt an mit mehr als einer Sprache aufwachsen.

Laut einer Statistik der Bundeszentrale für Politische Bildung ${ }^{1}$ von 2012 leben in Deutschland etwa 16 Mio. Personen, also knapp 20\% der Gesamtbevölkerung, mit Migrationshintergrund. In vielen deutschen Großstädten liegt die Anzahl an Schülerinnen und Schülern mit Migrationshintergrund bei bis zu einem Drittel der Gesamtschülerschaft. Auch sie leben - ebenso wie Moras Protagonistin - zwischen zwei Kulturen und mit zwei oder mehr Sprachen. Auch sie sind es gewohnt täglich zwischen zwei Welten und mindestens zwei Sprachen zu wechseln, oder diese zu vermischen, so wie es die Situation gerade erforderlich macht. Seit ihrer Geburt befinden sich die meisten unter ihnen im Spannungsgefüge sprachlicher, kultureller und sozialer Differenzen. Viele von ihnen kennen gesellschaftliche Anerkennung

1 Bundeszentrale für Polititsche Bildung (o.J.), Statistik: Migrantenanteil in deutschen Großstädten wächst. 
ebenso wie sie Ausgrenzung und Stereotypisierung kennen, und sie wissen um die enge Verknüpfung von Sprache, Identität und Schulerfolg.

Genau diesen Lernern widmet sich der hier vorliegende Beitrag. Er geht der Frage nach, wie Schule und vor allem der Englischunterricht mit den sprachlichen und kulturellen Erfahrungen mehrsprachiger Lerner umgehen sollte, um dem gerecht zu werden, was curriculare Vorgaben, wie etwa der Kernlehrplan für das Fach Englisch an Realschulen in NRW, einfordern: „Der lebensweltlichen Mehrsprachigkeit, die in den Klassenverbänden vorhanden ist, wird Rechnung getragen “ (Landesinstitut für Schule - NRW 2007).

Dazu soll zunächst die besondere Sprachlernsituation mehrsprachiger Lerner betrachtet werden mit gezieltem Blick auf die Frage, ob mehrsprachige Lerner beim Lernen einer weiteren Sprache Vor- oder Nachteile haben. Dabei lässt sich zunächst einmal feststellen, dass es keine pauschale Antwort auf diese Frage geben kann. Allerdings haben die verschiedenen Bedingungen, die sich aus den unterschiedlichen Sprachlernbiographien mehrsprachiger Lerner sowie ihrem häuslichen und schulischen Lernumfeld ergeben, einen bedeutenden Einfluss darauf, ob Mehrsprachige ihr „Mehr“ an Sprachbesitz im Sinne einer individuellen oder „lebensweltlichen Mehrsprachigkeit“ (Gogolin 2014: 416) zu ihrem Vorteil für das weitere Sprachenlernen nutzen können. Auf Basis dieses Wissens lassen sich letztlich didaktische Implikationen für die Inklusion von Herkunftssprachen im Fremdsprachenunterricht ableiten.

\section{Der mehrsprachige Sprachenlerner - ein potenzielles Sprachgenie?}

Das Vorhandensein lebensweltlicher Mehrsprachigkeit in der schulischen Praxis gestaltet sich für viele Kinder und Jugendliche in Deutschland weniger als Vorteil denn als Problem. Wirft man einen Blick auf die Ergebnisse einschlägiger Vergleichsstudien, wie PISA (PISA-Konsortium 2007, 2010) oder DESI (DIPF 2006, DESI-Konsortium 2008), so lässt sich erkennen, dass die schulischen Gesamtleistungen der Gruppe mehrsprachiger Schülerinnen und Schüler deutlich unter denen ihrer einsprachigen Mitschülerinnen und Mitschüler liegen. Begründet wird dieses Leistungsdefizit der mehrsprachigen Schülerinnen und Schüler sowohl mit ihren häufig erschwerten sozialen 
Lebensbedingungen als auch mit ihren unzureichenden (schrift-) sprachlichen Kompetenzen in ihrer Erst- und Zweitsprache (vgl. Ehlers 2014). Beide Faktoren sind dabei nicht wirklich unabhängig voneinander zu betrachten und man kann schlussfolgern: Wer in Deutschland mehrsprachig aufwächst, hat es in der Schule vermutlich schwer.

Umso erfreulicher, aber auch erstaunlicher, ist es in diesem Zusammenhang, wenn Experten aus der Fremdsprachendidaktik davon ausgehen, dass dies nicht für das Erlernen der schulischen Fremdsprachen zutrifft. Ganz im Gegenteil wird hier nicht selten postuliert, dass mehrsprachige Kinder und Jugendliche in den fremdsprachlichen Fächern sogar einen Vorteil gegenüber ihren einsprachigen Mitschülerinnen und -schülern haben. Die Begründung scheint einfach - man ist davon überzeugt, dass die bereits gemachten Sprachlernerfahrungen der Mehrsprachigen das Erlernen einer weiteren fremden Sprache grundsätzlich erleichtern (z.B. Böttger 2012: 12).

Neben dieser Hypothese, die letztlich besagt, dass Mehrsprachigkeit per se als positive Voraussetzung für das weitere Sprachenlernen gesehen werden kann, steht eine etwas abgeschwächte Position, die davon ausgeht, dass das Fremdsprachenlernen für mehrsprachige Schülerinnen und Schüler zumindest keinerlei Nachteil oder Überforderung darstelle (z.B. Börner 2009). Das tragende Argument dabei ist, dass alle Mehrsprachigen „zusammen mit allen anderen Schülerinnen und Schülern (in der Fremdsprache Englisch) am gleichen Punkt beginnen“ (Schmid-Schönbein 2008: 32) und damit dieselben Voraussetzungen für das Sprachenlernen haben.

Zusätzlich zu diesen Überlegungen findet man Arbeiten, die vor allem jüngeren Lernern mit Migrationshintergrund beim schulischen Englischlernen leichte Nachteile zuschreiben, insbesondere in den rezeptiven Fertigkeiten Hörverstehen und Lesen (z.B. Elsner 2007; Paulick \& Groot-Wilken 2009; Wilden \& Porsch 2015).

Vor dem Hintergrund dieser durchaus konträren Annahmen mit Blick auf die Frage, wie mehrsprachige Lerner im schulischen Fremdsprachenlernkontext einzuschätzen sind, schließen sich zwei Fragen an: a. Was ist dran an diesen Aussagen? Und b. Wie kommt es zu solchen unterschiedlichen Aussagen?

Im Folgenden sollen die o.g. Perspektiven deshalb genauer betrachtet und diskutiert werden. 
Vermutung 1: Mehrsprachige und Einsprachige haben dieselben Voraussetzungen beim Erlernen einer Fremdsprache

In Deutschland beginnen alle Kinder mit dem Fremdsprachenunterricht in der Grundschule. Am häufigsten wird dabei die Fremdsprache Englisch vermittelt. Diese ist für fast alle Kinder eine neue und damit unbekannte Sprache, die es zu erlernen gilt. Dies ist sicherlich eine gute Bedingung für ein- und mehrsprachige Kinder - insbesondere in emotionaler Hinsicht. Denn gerade Kinder mit einer anderen Muttersprache als der deutschen haben hier einmal nicht das Nachsehen, dass alles, was erklärt wird in der Muttersprache ihrer Mitschülerinnen und Mitschüler erklärt wird, jedoch nicht in ihrer. Insofern der Englischunterricht tatsächlich einsprachig in der Fremdsprache stattfindet, verstehen nun sowohl die deutschsprachigen Kinder als auch die nicht-deutschsprachigen Kinder erst einmal wenig oder nichts, dies ist ihnen allen auf jeden Fall allen gemein. Und in der Tat zeigen verschiedene Untersuchungen, dass die Leistungsunterschiede zwischen ein- und mehrsprachigen Fremdsprachenlernern im Fremdsprachenunterricht in vielen Fällen nicht signifikant sind (vgl. z.B. Özdemir 2006; Sanders \& Meijers 1995; van Gelderen et al. 2003) und deutlich geringer ausfallen als z.B. im Deutschunterricht (Köller, Knigge \& Tesch 2010). Dennoch stellt sich auch hier die Frage, wie man von vergleichbaren Ausgangspunkten oder Voraussetzungen sprechen kann, wenn sich die Tatsache nicht abstreiten lässt, dass den Mehrsprachigen bereits zu Beginn des schulischen Fremdsprachenunterrichts mehr Sprachen zur Verfügung stehen als den Einsprachigen und sie Sprachlernerfahrungen mit mehreren Sprachen gesammelt haben, über die die Einsprachigen nicht verfügen. Bei dieser Argumentation scheint es demnach völlig irrelevant zu sein, dass die mehrsprachigen Fremdsprachenlerner ihre bereits vorhandenen Sprachen auf äußerst unterschiedlichen Kompetenzniveaus beherrschen und nicht relevant, wie viele negative oder auch positive Erfahrungen sie im Zweifel bereits beim Erlernen ihrer zweiten Sprache Deutsch gemacht haben.

Dass diese Erfahrungen häufig einschneidend sind und vermutlich wenig hilfreich für das weitere Fremdsprachenlernen, lässt sich aus dem folgenden Zitat Mahmuts, einem türkisch-deutschen Schüler einer 11. Jahrgangsstufe, leicht ableiten: 
My first language is Turkish. While I entered school, I was in the german lesson one of the worst pupils. It was a bad feeling. I was felt outside. ${ }^{2}$ (Mahmut, bilingual, L1 Türkisch, L2 Deutsch)

Im Gegensatz dazu scheint sein monolingualer Mitschüler Mark keine solchen negativen Erfahrungen gemacht zu haben; zu seiner Sprachlernerfahrung und seinen vorhandenen Sprachen äußert er sich wie folgt:

I speak German $[\ldots]$ and we speak standard German not like the bavarians which speak bavarian. (Mark, monolingual, L1 Deutsch)

Wenig überraschend haben die beiden äußerst unterschiedliche Sprachenselbstkonzepte in Bezug auf ihre erste schulische Fremdsprache Englisch sowie ihrer zweiten schulischen Fremdsprache Spanisch.

So verweist Mahmut bei der Beantwortung der Frage How do you feel, when you speak English or Spanish? auf seine Ängste:

I am feel not so good because I haven't a good command of these languages. I am afraid when the people speak the language fast, because I don't understand anything. (Mahmut, bilingual, L1 Türkisch, L2 Deutsch)

Mark hingegen zeigt ein hohes Selbstkonzept in Bezug auf seine fremdsprachlichen Kompetenzen in Englisch:

When I speak English I feel good, because I can speak English very well. And I'm not afraid of speaking English. (Mark, monolingual, L1 Deutsch)

Auch wenn die beiden Lernenden hier lediglich ihre Selbsteinschätzung in Bezug auf ihre sprachlichen Kompetenzen wiedergeben und nichts über etwaige Ergebnisse in Leistungstests angeben, mutet die Annahme, dass der Fremdsprachenunterricht für Mehrsprachige und Einsprachige stets dieselben Bedingungen bereit hält, doch etwas seltsam an vor dem Hintergrund solcher Aussagen. Das Argument, dass alle Lerner dieselben Voraussetzungen im Fremdsprachenunterricht haben, weil sie alle die zu erlernende

2 Das hiesige und die nachfolgenden Schülerzitate entstammen einer im Herbst 2009 durchgeführten schriftlichen Befragung von 23 Schülern einer 11. Jahrgangsstufe an einem Bremer Gymnasium. Die Namen der Lerner wurden geändert, die Rechtschreibung entspricht den Originalzitaten der Befragten. 
Sprache noch nicht kennen, ist vergleichbar mit dem Argument, dass ein gedopter Sprinter genauso gute Chancen hat zu gewinnen wie ein nicht gedopter Läufer, denn schließlich stehen ja beide an derselben Startlinie und haben dieselbe Strecke zu laufen.

Der Argumentation, dass ein- und mehrsprachige dieselben Chancen im Fremdsprachenunterricht haben, liegt demnach ein eher veraltetes Bild mehrsprachiger Sprachlernprozesse zugrunde, welches annimmt, dass Sprachen separat voneinander gelernt und in getrennten Schubladen gespeichert werden. Bei dieser Annahme wird offensichtlich vergessen, dass emotionale Erfahrungen, die Lernende beim Sprachenlernen machen, einen starken Einfluss darauf haben können, wie erfolgreich das Lernen weiterer Sprachen verläuft.

Vermutung 2: Mehrsprachige Lerner sind die besseren Fremdsprachenlerner Dass mehrsprachige Lerner die besseren Fremdsprachenlerner sind, lässt sich auf der Grundlage von Erkenntnissen aus Untersuchungen unterschiedlicher Forschungsdisziplinen vermuten. Dies sind erstens neurolinguistische Untersuchungsverfahren zum multiplen Spracherwerb, zweitens psycholinguistische Erkenntnisse aus der Drittspracherwerbsforschung und drittens die Ergebnisse aus der empirischen Unterrichtsforschung.

\section{Ergebnisse der Neurolinguistik}

Während man früher der Ansicht war, dass unterschiedliche Sprachen auch in unterschiedlichen Hirnregionen angesiedelt sein müssten, weiß man heute, dass sich die verschiedenen Sprachen im Gehirn überlagern. Allerdings wird die Repräsentation der Sprachen durch verschiedene Faktoren beeinflusst, zu denen zunächst das Erwerbsalter in den jeweiligen Sprachen gehört (vgl. Weskamp 2007: 53). So zeigen die während der Computertomographie aufgezeichneten Aktivierungsmuster für die vorhandenen Sprachen bei Mehrsprachigen, die in den ersten 6 Lebensjahren parallel mit zwei oder mehr Sprachen aufwachsen, im Broca-Areal weitgehende Überlappung, wohingegen die Sprachen im Gehirn bei Spätmehrsprachigen keine so kompakte Repräsentation mehr aufweisen wie dies bei den Frühmehrsprachigen der Fall ist (vgl. z.B. Franceschini 2002).

Menschen, die früh mit zwei Sprachen aufwachsen verfügen demnach über ein spezielles Sprachennetzwerk im Gehirn, in das später auch weitere 
Sprachen leicht integriert werden können. Man könnte aus neurolinguistischer Sicht somit tatsächlich vermuten, dass sich frühe Mehrsprachigkeit grundsätzlich positiv auf die Sprachaneignung weiterer Sprachen auswirkt und es mehrsprachigen Kindern und Jugendlichen, die früh mit zwei oder mehr Sprachen in Berührung kommen, in unserer Schule leichter fällt, eine dritte oder vierte Sprache zu erlernen.

\section{Erkenntnisse der Psycholinguistik}

Diese neurolinguistischen Ergebnisse werden gestützt durch psycholinguistische Modelle aus der Tertiärsprachenforschung, welche annehmen, dass Lernersprachen miteinander interagieren und mehrsprachige Lerner bei der Aneignung einer weiteren Sprache implizit auf die bereits gespeicherten sprachlichen Wissensbestände zurückgreifen (vgl. u.a. Dégi 2010; Gibson \& Hufeisen 2007; Hufeisen 2003), um sich z.B. neuen Wortschatz oder fremde Texte zu erschließen. Auch ist mittlerweile bekannt, dass Mehrsprachige über eine höhere Anzahl an Wörtern verfügen als Einsprachige, jedoch ist dieses lexikalische Wissen über zwei Sprachen verteilt (vgl. Poarch 2013), und es ist wahrscheinlich, dass die Mehrsprachigen in den einzelnen Sprachen über einen geringeren Wortschatzbestand verfügen als gleichaltrige Einsprachige. Mit Sicherheit jedoch verfügen Mehrsprachige über eine erhöhte metalinguistische Bewusstheit, die z.B. in einer häufigeren Anwendung von Transfer- oder Erschließungsstrategien im Bereich Lexik und Grammatik zum Ausdruck kommt (z.B. Bialystok 2009) und welche sich beim Lernen einer Fremdsprache günstig auswirken kann.

Trotz dieser - aus neuro- und psycholinguistischer Perspektive betrachteten - guten Voraussetzungen, die Mehrsprachige mit in den Unterricht bringen, ist die Erkenntnislage in puncto „mehrsprachige Fremdsprachenlernerfolge“ jedoch alles andere als eindeutig, wie der nachfolgende Absatz zeigt.

Vermutung 3: Mehrsprachige Lerner schneiden im Fremdsprachenunterricht schlechter ab als Einsprachige

So zeigen die Ergebnisse aus verschiedenen Untersuchungen zum Vergleich der fremdsprachlichen Kompetenzen von ein- und mehrsprachigen Schülerinnen und Schülern an Grundschulen u.a., dass Kinder, die mit einer anderen 
Sprache als der Deutschen zuhause aufwachsen, weniger Punkte in den Leistungstests im Bereich Hören, Lesen und Schreiben in der Fremdsprache Englisch erzielen als ihre einsprachigen Mitschülerinnen und -schüler (z.B. Elsner 2007; May 2006; Paulick \& Groot-Wilken 2009; Wilden \& Porsch 2015):

Zwischen Kindern mit deutscher und Kindern mit anderen Herkunftssprachen gibt es in den gezeigten Leistungen durchaus Unterschiede. Kinder aus deutschsprachigen Familien (1336 Schülerinnen und Schüler) erzielten einen mittleren Punktwert von 30,5. Kinder, die nach Aussagen der Fachlehrkräfte bilingual aufwachsen (174 Schülerinnen und Schüler) erreichen im Durchschnitt 28,5 Punkte, Kinder mit anderen Herkunftssprachen (238 Schülerinnen und Schüler) im Mittel einen Punktwert von 25,7. (Groot-Wilken, Engel \& Thürmann 2007)

Dabei zeigen die Studien jedoch auch, dass es zwischen den einzelnen Sprachgruppen ebenso deutliche Unterschiede gibt wie zwischen Kindern, die die deutsche Sprache mit unterschiedlicher Intensität verwenden. Während sowohl in der EVENING Studie (Engel, Groot-Wilken \& Thürmann 2009) als auch in der DESI Studie (DESI-Konsortium 2008) z.B. russische Schülerinnen und Schüler nur geringfügig schlechtere Leistungen erzielen als ihre monolingual-deutschsprachigen Mitschülerinnen und Mitschüler, liegen die türkischen Schülerinnen und Schüler in diesen Studien stets auf dem letzten Platz (vgl. Göbel, Rauch \& Vieluf 2011). Zudem scheint es einen Unterschied zu machen, ob die Mehrsprachigen zuhause neben einer anderen Sprache auch die Schul- und Umgebungssprache sprechen, ob sie muttersprachlichen Ergänzungsunterricht erhalten oder ob im häuslichen Umfeld ganz andere Sprachen gesprochen werden als in der Schule - wobei bislang wenig eindeutig ist, welche dieser Varianten sich für das fremdsprachliche Lernen tatsächlich als vorteilhaft herausstellt (vgl. Elsner 2007; Wilden \& Porsch 2015).

Zusammenfassend zeigen diese Ergebnisse jedenfalls, dass Mehrsprachige nicht grundsätzlich die besseren Fremdsprachenlerner sind, einige von ihnen aber gute Voraussetzungen dafür mitbringen, während andere dies wiederum nicht tun. Entsprechend lassen diese Ergebnisse vor allem eine Schlussfolgerung zu, nämlich diejenige, dass es den oder die Mehrsprachige/n ebenso wenig gibt wie die oder den Einsprachige/n, sondern Mehrsprachigkeit verschiedene Ausprägungen haben kann, die durch unterschiedliche Bedingungen entstehen. Welche Bedingungen dies sind, soll im Folgenden erläutert werden. 


\section{Bedingungen von Mehrsprachigkeit und ihre Bedeutung für das Fremdsprachenlernen}

Neben dem Erwerbsalter sind mindestens vier weitere Faktoren ausschlaggebend dafür, wie sich vorhandene Sprachenkenntnisse und gemachte Sprachlernerfahrungen auf den weiteren Sprachlernprozess auswirken. Dies sind die sozioökonomischen Verhältnisse der Lerner gekoppelt mit der Bildungsnähe und Unterstützung des Elternhauses, die sprachliche Kompetenz der Lerner in ihren vorhandenen Sprachen, die eigene Einstellung zur vorhandenen Mehrsprachigkeit sowie das Angebot der Lernumgebung. Im Folgenden werden diese Bedingungsfaktoren näher betrachtet.

\section{Sozioökonomischer Hintergrund, Bildungsnähe und Unterstützung des Elternhauses}

In einer Re-Analyse der DESI Studie (Göbel et al. 2011) konnte gezeigt werden, dass der sozioökonomische Hintergrund, die Bildungsnähe und die Unterstützung des Elternhauses einen großen Einfluss auf die sprachliche Entwicklung der ein- und mehrsprachigen Lerner haben - dies gilt auch für die Entwicklung in der Fremdsprache. Mit anderen Worten, wer aus einem sozial abgesicherten und bildungsnahen Elternhaus kommt und mehrsprachig aufwächst, hat Vorteile beim Sprachenlernen. Fakt ist jedoch, dass viele mehrsprachige Schülerinnen und Schüler mit Migrationshintergrund aus bildungsfernen und sozial benachteiligten Elternhäusern stammen und deshalb nicht die entsprechende Unterstützung in ihrer Entwicklung erfahren, die sie bräuchten, um von ihrer Mehrsprachigkeit profitieren zu können (vgl. Bundesamt für Migration und Flüchtlinge ${ }^{3}$ 2012). Daran können auch Lehrkräfte kurzfristig nicht wirklich etwas ändern. Es bleibt jedoch zu hoffen, dass sich die zunehmend besseren Bildungsbedingungen für Migranten und speziell darauf abgestimmte Förderinitiativen langfristig auch in einem höheren sozioökonomischen Status niederschlagen, der sich wiederum positiv auf die sprachliche Entwicklung der Kinder auswirken wird.

3 Bundesamt für Migration und Flüchtlinge (2012), Migrationsbericht des Bundesamtes für Migration und Flüchtlinge im Auftrag der Bundesregierung. 


\section{Kompetenzen in Erst- und Zweitsprache}

In der o.g. Re-Analyse der DESI Studie (Göbel et al. 2011) konnte ebenfalls eruiert werden, dass der sozioökonomische Hintergrund bei den türkischen Kindern keinen so entscheidenden Einfluss auf die sprachlichen Leistungen der Lerner hatte wie der Faktor Literalität. D.h. auch mehrsprachige Kinder aus sozial benachteiligten Familien können in der Fremdsprache von ihrer Mehrsprachigkeit profitieren, solange sie in der deutschen Sprache oder / und in ihrer Erstsprache über hohe (schriftsprachliche) Kompetenzen verfügen. Doch auch hier muss bedacht werden, dass viele mehrsprachige Schülerinnen und Schüler weder in ihrer Erstsprache noch in der Zweitsprache ausreichend Zugang zu Büchern oder Zeitschriften haben - entsprechend lernen sie in ihrer Erstsprache vielfach nicht zu lesen oder zu schreiben. Die Alphabetisierung erfolgt erst in der Schule und hier zunächst nur in der Zweitsprache. Spätestens seit den Untersuchungen von Jim Cummins Ende der 1980er Jahre wissen wir aber, dass eine hohe Kompetenz in der Erstsprache bei gleichzeitiger Förderung der Zweitsprache eine wichtige Voraussetzung dafür ist, dass man seine Mehrsprachigkeit für das weitere Sprachenlernern optimal nutzen kann, während eine Nichtförderung der Erstsprache negative Folgen sowohl für den allgemeinen kognitiven und den sprachlichen Entwicklungsprozess als auch für die Identitätsbildung der Kinder haben kann (vgl. Apeltauer 2007; Peltzer-Karpf 2003: 448). Eine hohe Sprachkompetenz zeichnet sich sowohl durch basale mündliche, alltagssprachliche Kompetenzen aus (BICS) als auch durch schriftsprachliche Kompetenzen und einer differenzierten Sprachform im Sinne einer CALP / Cognitive Academic Language Proficiency (Cummins \& Swain 1986).

Es lässt sich damit annehmen, dass vor allem solche Kinder von ihrer mehrsprachigen Kompetenz im weiteren Sprachlernprozess profitieren können, die kontinuierlich in ihrer Erst- und Zweitsprache gefördert werden (vgl. Lohmann 2008: 26) und die somit über hohe (schriftsprachliche) Kompetenzen in ihren vorhandenen Sprachen verfügen. Bislang jedoch bleibt die Inklusion von Herkunftssprachen im Sinne einer solchen systematischen Förderung ein Desiderat, dem derzeit nur wenige Schulen nachkommen. 


\section{Einstellungen der Mehrsprachigen zu ihrer eigenen Mehrsprachigkeit}

Ein weiterer wesentlicher Faktor für mehrsprachigen Lernerfolg zeigt sich in den eigenen Einstellungen der Mehrsprachigen zu ihrer vorhandenen Mehrsprachigkeit. So kommen Gibson und Hufeisen zu folgendem Schluss:

Lernende, die sich ihrer Mehrsprachigkeit bewusst sind, die sie akzeptieren und konstruktiv nutzen, d.h. solche, die sich Gedanken über ihre verschiedenen Sprachen und ihr Miteinanderwirken machen, gehen souveräner mit dem Lernprozess in der Fremdsprache um. Sie entwickeln individuelle Lernstrategien und Lernweisen. (Gibson \& Hufeisen 2007: 38)

Viele mehrsprachige Schülerinnen und Schüler mit Migrationshintergrund bewerten ihre individuelle Mehrsprachigkeit im Kontext von Schule und Unterricht oft eher als hinderlich denn als hilfreich: So erklärt auch Bilat, deren Muttersprache Türkisch ist:

To speak 2 languages is not so nice, because i can't learn none of those perfect. It's difficult to speak both languages at the same time and place. The positive thing is, that i communicate with germans and turkisch people. (Bilat, bilingual, L1 Türkisch, L2 Deutsch)

In Bezug auf ihre Kompetenzen in den Schulfremdsprachen Englisch und Spanisch berichtet sie von Unsicherheit und der Angst, Wörter zu vermischen:

I fell me insecure. [...] the problem is to mix up any words. (ebd.)

Und damit steht sie nicht alleine da - auch Eyzon, ihr Klassenkamerad, erklärt auf die Frage, wie er sich fühlt, wenn er Englisch oder Spanisch spricht, dass er nervös sei und sich irgendwie „anders“ fühle.

Schon 2007 stellte Natalia Portnaia in einer Untersuchung mit russischdeutschen Fremdsprachenlernern fest, dass viele Schülerinnen und Schüler ihre gemachten Sprachlernerfahrungen nicht immer als etwas wahrnehmen, das ihnen das weitere Fremdsprachenlernen erleichtern könnte:

Im schulischen Kontext ist die Sprachmischung jedoch eher mit einem negativen Gefühl verbunden. Das Durcheinanderkommen kann von anderen bemerkt werden - man wird in seinem sprachlichen Können eingeschätzt und hat Angst vor einer Blamage. (Portnaia 2007: 116) 
So erklärt Sarah, ein polnisch-deutsches Mädchen:

My mother tongue is polish. I'm afraid when speaking another language sometimes. Me dearta sometimes words... and I cannot the syntax. (Sarah, bilingual, L1 Polnisch, L2 Deutsch)

Dieses offensichtliche „Chaos im Kopf“, das die verschiedenen Sprachen im Unterricht häufig hervorzurufen scheinen, führt hier offensichtlich zu einer Angst vor dem Sprechen und letztlich dazu, dass die Mehrsprachige tatsächlich keinen Gewinn aus ihrer Mehrsprachigkeit für den Fremdsprachenunterricht ziehen kann.

Lernende [...] die ängstlich oder ärgerlich auf ihr Chaos der verschiedenen Sprachen im Kopf verweisen, produzieren tatsächlich auch zahlreiche Interferenzen und nutzen das Potenzial ihrer Mehrsprachigkeit nicht, sondern ignorieren sie oder machen sie sich nicht bewusst, um sie in konstruktiver Weise zu nutzen. (Gibson \& Hufeisen 2007: 38)

Wie Gibson und Hufeisen jedoch auch klarstellen, muss man dies aber nicht den Mehrsprachigen selbst vorwerfen, denn „[...] oft wissen sie es gar nicht, weil sie nicht darauf hingewiesen bzw. angeleitet werden, ihr persönliches Mehrsprachigkeitsprofil mit einem Set an Strategien, die für sie funktionieren, zu entwickeln und diese dann gezielt einzusetzen“ (ebd.). Damit wird klar, dass die eigene Einstellung zur Mehrsprachigkeit in engem Zusammenhang mit der Sprachlernumgebung steht und damit, was diese den Mehrsprachigen anbietet, um ihre mehrsprachigen Kenntnisse aktiv und gewinnbringend nutzen zu können.

\section{Bedeutung der Sprachlernumgebung}

Die Europäische Kommission hat die Förderung und Anerkennung von Mehrsprachigkeit durch die Inklusion von Herkunftssprachen sowie den von mehrsprachigen Lernern gemachten Sprachlernerfahrungen bereits im Jahr 2006 vorgeschlagen:

Education systems need to ensure the harmonious development of learners' plurilingual competence through a coherent, transversal and integrated approach that takes into account all the languages in learners' plurilingual repertoire and their respective functions. This includes promoting learners' consciousness of their existing repertoires and potential to develop and adapt those repertoires to changing circumstances. (Council of Europe 2006: 5) 
Die Ergebnisse einer qualitativen Studie, im Rahmen welcher an einer Frankfurter Gesamtschule Englischlehrkräfte dazu befragt wurden, ob und gegebenenfalls wie sie die Mehrsprachigkeit ihrer Schülerinnen und Schüler im Unterricht integrieren, zeigt jedoch, dass wir von einem sprachentransversalen Ansatz wie ihn das Europäische Papier vorschlägt noch weit entfernt sind. So beteuern die befragten Lehrkräfte: „, [Mehrsprachigkeit] wird weder negiert [...] noch in irgendeiner Form nutzbar gemacht. [...] es stört keinen weiter, aber es freut auch keinen sonderlich. “ (C.L. in Kollmeyer 2007: 262).

Den subjektiven Theorien der meisten Fremdsprachenlehrkräfte zufolge stellt die besondere Bildungsvoraussetzung von Schülern mit Migrationshintergrund auch weder einen Vor- noch einen Nachteil dar. Die Herkunftssprachen werden deshalb nicht in den Englischunterricht mit einbezogen. Die Basis des Fremdsprachenlernens sei Deutsch, denn diese Sprache sei schließlich auch näher am Englischen dran als die Herkunftssprachen. Zudem könne man auf diese auch deshalb nicht Bezug nehmen, weil man „[...] auf die Nutzung der in der Klasse vertretenen Sprachen nicht vorbereitet sei.“ (Kollmeyer 2007: 262).

So subsumiert Adelheid Hu treffend:

Während für die Schüler/innen Mehrsprachigkeit und sprachlich-kulturelle Identität zentrale Kategorien darstellten, spielten diese für die Fremdsprachenlehrer/ innen kaum eine Rolle. (Hu 2010: 67)

Offensichtlich handelte es sich um eine fest verwurzelte fremdsprachendidaktische Tradition, alle anderen im Klassenverband auftauchenden Sprachen - bis auf das Deutsche - prinzipiell auszuschließen, eine monolinguale Lerngruppe vorauszusetzen und Sprachen darüber hinaus als ein Phänomen anzusehen, das mit der Emotionalität und der Identität der Lernenden nicht verbunden ist. (Hu 2010: 68)

Wie Singleton und Aronin (2007) hervorheben, lassen sich die besonderen Voraussetzungen, die mehrsprachige Lerner mit in den Unterricht bringen letztlich aber nur dann im Fremdsprachenunterricht gewinnbringend nutzen, wenn die Lernsituation dies auch zulässt - oder besser noch - erforderlich macht.

The experiential profile of multilingual learners provides them with especially favourable conditions to develop awareness for the social and cognitive possibilities which their particular situation affords them. (ebd. 2007: 83) 
Im Kontext von Schule und (Fremd-) Sprachenunterricht lässt sich dies meiner Ansicht nach auf drei Ebenen verwirklichen, nämlich auf der systemischen Ebene der jeweiligen Bildungseinrichtung, auf der Unterrichtsebene, auf der individuellen Ebene. Auf diese drei Ebenen soll im Folgenden kurz eingegangen werden.

\section{Inklusion von Mehrsprachigkeit}

\section{...auf der systemischen Ebene}

Der europäischen Forderung nach einer Erziehung zur „Mehrsprachigkeit“ wird nicht allein dadurch Rechnung getragen, dass moderne Fremdsprachen im Bildungssystem verankert sind. Vielmehr müssen auch die vorhandenen Muttersprachen der Kinder in Form von Ergänzungsunterricht oder im Rahmen von AGs einbezogen werden. Solche Maßnahmen erscheinen vor dem Hintergrund der sprachlichen Vielfalt, der wir an vielen Schulen begegnen, allerdings nur schwer umsetzbar zu sein. Letztlich scheitert es daran, dass es nicht genügend Lehrkräfte gibt, die solche Angebote machen können. Hier muss überlegt werden, ob Angebote dieser Art von älteren Schülern oder Eltern geleitet werden können oder ob es Studierende gibt, die etwa erstsprachliche Förderkurse an Schulen offerieren können. Besonders positiv zu bewerten sind zudem Förderprogramme, die mehrsprachige Lerner in ihrer sprachlichen Entwicklung in der Bildungssprache Deutsch unterstützen. Zu nennen sind hier vor allem die Großprojekte der Stiftung Mercator aber auch kleinere Projekte, wie z.B. das Bremer Projekt Enter ${ }^{4}$, das beispielhaft zeigt, wie Eltern und Familien in die alltägliche Bildungsarbeit einbezogen werden können, um die sprachlichen Kompetenzen von Kindern im Vorschulalter zu fördern.

Auch auf universitärer Ebene muss Mehrsprachigkeit stärker in den Fokus rücken, erstens in der Lehrerbildung, in der Studierende auf den Umgang mit sprachlicher Heterogenität vorbereitet werden müssen (vgl. hierzu Überlegungen von Wildemann, Esteve, Hoodgarzadeh \& Walter 2014) und zweitens übergreifend mit Blick auf die Förderung von Studierenden mit

4 Weitere Informationen zum Projekt findet man unter: http://www.fb12.unibremen. de/fileadmin/Arbeitsgebiete/deutsch/Werke/Boenig Hering Thoene 2014 ENTER IMPULSE.pdf, letzter Aufruf 21.12.2014. 
mehrsprachigem Hintergrund. Auch hier macht sich die Stiftung Mercator ${ }^{5}$ stark, z.B. im Rahmenprogramm Studium+M, das Projekte fördert, die zur Verbesserung der Chancengleichheit für Studierende mit Migrationshintergrund beitragen.

\section{... auf der Unterrichtsebene}

Neben diesen Möglichkeiten zur Inklusion von Muttersprachen auf systemischer Ebene gilt der Fremdsprachenunterricht seit jeher als der Türöffner zu mehrsprachigen Bildungsprozessen. Nimmt man die Forderung nach einer Erziehung zur Mehrsprachigkeit des Europarates ernst, so muss der fremdsprachliche Unterricht nicht nur die in monofachlicher Hinsicht jeweils angestrebte schulische Fremdsprache ausbilden, sondern darüber hinaus so oft wie möglich explizite Bezüge zu den bereits gelernten Sprachen herstellen und die Sprachlernerfahrungen der Lerner miteinbeziehen. Dies kann sowohl in inhaltlich-thematischer Hinsicht geschehen als auch methodischmedial. So können, z.B. Texte, die das Thema Mehrsprachigkeit und Transkulturalität aus der Innenperspektive mehrsprachiger Individuen bearbeiten - wie z.B. die Graphic Novel „Indian by Choice“ (Amit Dasgupta), in der es um einen amerikanischen Jugendlichen geht, der seine indischen Wurzeln zunächst leugnet und schließlich schätzen lernt, oder „American Born Chinese“ (Gene Young), in der ein amerikanisch-chinesischer Teenager in einer bilingualen und bikulturellen Identitätskrise steckt, die Lerner dazu anregen über ihre eigene sprachliche und kulturelle Identität nachzudenken. Dazu eignen sich neben Graphic Novels, Filmen, Kurzgeschichten und Romanen auch mehrsprachige Gedichte besonders gut, mit denen sich Fragen ergeben wie: In welcher Sprache hat der Autor / die Autorin seine Ideen ausgedrückt und weshalb? Solche Überlegungen geben Anlass zur eigenen Produktion von mehrsprachigen Gedichten und zur Reflexion darüber, was man selbst in welcher Sprache am besten beschreiben kann (vgl. hierzu Elsner 2011).

Will man weniger über die individuelle Sprachenwahl und sprachliche Identität reflektieren als vielmehr Texte in verschiedenen Sprachen anbieten, um sprachliche Vorerfahrungen zu nutzen oder mehrsprachige Kenntnisse

5 Weitere Informationen zum Projekt findet man unter: https://www.stiftungmercator.de/media/downloads/1 Nachrichten/2014 07/Studium Migrationshintergrund Presseinfo Juli 2014.pdf, letzter Aufruf 21.12.2014. 
weiter zu fördern, eignen sich vor allem digitale tools. Besonders hilfreich sind multimodale Angebote, die neben der schriftlichen Variante auch die auditive Erschließung eines Texts ermöglichen. Neben mehrsprachigen Wörterbüchern mit integrierter Audiofunktion eignen sich hier vor allem Wörterbücher, die mit einem Tiptoy oder Reading Pen (elektronische Lesestifte) geliefert werden und den Schülern so die autonome Nutzung vorhandener Sprachen ermöglichen. Längere Texte in vielen Sprachen, auf die parallel zugegriffen werden kann, bietet z.B. das Lernprogramm „My first Stories“ (Oldenbourg Verlag).

\section{...auf der individuellen Ebene}

Die Überlegungen auf dieser Ebene beziehen sich auf die affektiv-reflexive Auseinandersetzung sowie die subjektiven Theorien zur eigenen Sprachlernbiographie: Sprachenlernen ist ein höchst emotionaler und individueller Prozess. Die Sprachlernerfahrungen der Lerner, ihre Selbstkonzepte und Einstellungen gegenüber ihren vorhandenen und anderen Sprachen sind ein wichtiger Bestandteil der eigenen Identität und müssen entsprechend im Fremdsprachenunterricht mitberücksichtigt werden. Der Fremdsprachenunterricht sollte deshalb ausreichend Reflexionsraum bieten, in welchem Sprachlernerfahrungen, Sprachbedürfnisse, Einstellungen zur eigenen Mehrsprachigkeit und der Erfolg der bislang verwandten Lernstrategien punktuell thematisiert werden können. Hilfreich sind hier insbesondere Sprachenportfolios, Lerntagebücher und Lautdenkmethoden.

Positiv hervorzuheben sind solche Unternehmungen, in denen Kinder, Eltern und Erzieher gemeinsam an der zweitsprachlichen Literalitätsförderung mehrsprachiger Kinder arbeiten (vgl. z.B. Bönig, Hering \& Thöne 2014), in einem Folgeschritt sollte diese Förderung auch für die Erstsprache der Kinder angedacht werden.

\section{...auf der Ebene der Lehrkräfte}

Was für die Inklusion von Menschen mit körperlicher oder geistiger Behinderung gilt, gilt auch für die Inklusion von Sprachen: Ein wesentlicher Erfolgsfaktor liegt im Willen der Lehrkraft bzw. Erzieherin (vgl. Alechina, Alekseeva \& Agafonova 2011). Nur wenn Lehrkräfte selbst beginnen die Inklusion von vielen Sprachen als wichtigen Bestandteil ihres Unterrichts 
anzuerkennen, kann eine Erziehung zur Mehrsprachigkeit gelingen. Im Fremdsprachenunterricht empfiehlt es sich, dass Lehrkräfte den Schülerinnen und Schülern die Arbeit mit dem Portfolio nahe legen. Mit diesem Instrument der Selbsteinschätzung werden sich die Lerner selbst - aber auch ihre Lehrkräfte - über ihren Sprachstand und ihre sprachliche Entwicklung bewusst. Lerner sollten insgesamt häufiger dazu animiert werden, ihre vorhandenen Sprachen zu thematisieren, zu nutzen und miteinander zu vergleichen. Sprachbewusstseinsprozesse können nur dann in Gang gesetzt werden, wenn Sprachvergleiche im Unterricht nicht nur erlaubt sondern offiziell erwünscht sind. Wenn Schülerinnen und Schüler schließlich erkennen, dass ihre Lehrkräfte Interesse an ihren Erstsprachen haben, dann werden auch die Lerner selbst bald eine andere Einstellung zu ihren sprachlichen Kompetenzen entwickeln (vgl. Lohe \& Elsner 2014).

\section{Fazit}

„A language-friendly environment is an environment where different languages are heard and seen, where speakers of all languages feel welcome and language learning is encouraged", erklärt die Europäische Kommission im Jahr 2008. Eine solche sprachenfreundliche Umgebung bezieht Herkunftssprachen mit ein und lässt die aktive Auseinandersetzung mit Sprachlernerfahrungen zu. Insbesondere der fremdsprachliche Unterricht kann zu einer solchen Umgebung werden und im Kontext von Inklusion ein Zeichen setzen, indem er nicht nur die in monofachlicher Hinsicht jeweils angestrebte schulische Fremdsprache ausbildet, sondern darüber hinaus so oft wie möglich explizite Bezüge zu den bereits gelernten Sprachen und den Sprachlernerfahrungen der Lerner herstellt.

\section{Literaturverzeichnis}

Apeltauer, Ernst (2007), Anbahnen von Biliteralität bei Vorschulkindern mit

Migrationshintergrund. In: Elsner, Daniela; Küster, Lutz; Viebrock, Britta (Hrsg.) (2007), Fremdsprachenkompetenzen für ein wachsendes Europa. Das Leitziel Multiliteralität. Frankfurt am Main: Peter Lang, 195-214. Alechina, S.V.; Alekseeva, M.N. \& Agafonova E.L. (2011), Willingness of teachers as the main factor of success in the formation of the inclusive. Psychological Science and Education: 83-92. 
Bönig, Dagmar; Hering, Jochen \& Thöne, Bernadette (2014), Kinder entern Sprache und Mathematik mit der Schatzkiste [Online: http://www.fb12. uni-bremen.de/fileadmin/Arbeitsgebiete/deutsch/Werke/Boenig_Hering Thoene 2014 - ENTER IMPULSE.pdf 15.12.2014].

Börner, Otfried (2009), Fremdsprachenlernen in der Grundschule: die Hamburger KESS-Studie. In: Engel, Gaby; Groot-Wilken, Bernd \& Thürmann, Eike (Hrsg.) (2009), Englisch in der Primarstufe - Chancen und Herausforderungen. Berlin: Cornelsen, 67-75.

Bundeszentrale für Polititsche Bildung (o.J.), Statistik: Migrantenanteil in deutschen Großstädten wächst [Online: http://www.bpb.de/gesellschaft/ migration/newsletter/148820/migrantenanteil-in-deutschen-grossstaedtenwaechst 12.12.2014].

Bundesamt für Migration und Flüchtlinge (Hrsg.) (2012), Migrationsbericht des Bundesamtes für Migration und Flüchtlinge im Auftrag der Bundesregierung. Migrationsbericht 2012 [Online: http://www.bamf.de/ SharedDocs/Anlagen/DE/Publikationen/Migrationsberichte/migrationsbericht-2012.pdf? blob=publicationFile 15.12.2014].

Bialystok, Ellen (2009), Bilingualism: The good, the bad, and the indifferent. Bilingualism: Language and Cognition 12: 1, 3-11.

Bos, Wilfried \& Pietsch, M. (Hrsg.) (2005), KESS 4 - Kompetenzen und Einstellungen von Schülerinnen und Schülern am Ende der Jahrgangstufe 4 in Hamburger Grundschulen. Münster: Waxmann.

Bos, Wilfried; Hornberg, S.; Arnold, K.-H.; Faust, G.; Friede, L.; Lankes, E.-M.; Schwippert, K. \& Valtin, R. (Hrsg.) (2008), IGLU-E 2006 - Die Länder der Bundesrepublik Deutschland im nationalen und internationalen Vergleich. Münster / New York / München / Berlin: Waxmann.

Böttger, H. (2012), Voraussetzungen von Grundschulkindern für den Fremdsprachenerwerb. Englisch. Didaktik für die Grundschule. Berlin: Cornelsen, 8-13.

Cenoz, Jasone (2003), The additive effect of bilingualism on third language acquisition: A review. International Journal of Bilingualism 7: 1, 71-87.

Commission of the European Communities (2003), Communication from the Commission to the Council, the European Parliament, the Economic and Social Committee and the Committee of the Regions. Promoting Language Learning and Linguistic Diversity: an Action Plan 2004-2006: COM, 449 final. Brüssel. 
Commission of the European Communities (2008), Communication from the Commission to the Council, the European Parliament, the Economic and Social Committee and the Committee of the Regions. Multilingualism: An asset for Europe and a shared commitment: COM, 566 final. Brüssel.

Council of Europe (2008), Council conclusions of 22 May 2008 on Multilingualism. Official Journal of the European Union: C 140, 14-15.

Cummins, Jim (1987), Bilingualism, language proficiency, and metalinguistic development. In: Homel, P.; Palij, M \& Aaronson, D. (Hrsg.) (1987), Childhood Bilingualism. Aspects of Linguistic, Cognitive, and Social Development. Hillsdale, N.J.: Erlbaum, 57-73.

Cummins, Jim (1991), Interdependence of first- and second language proficiency. In: Bialystok, Ellen (Hrsg.) (1991), Language Processing in bilingual children. Cambridge, U.K: Cambridge University Press, 1-43.

Dégi, Zsuzanna (2010), Effect on the Linguistic Awareness of Foreign Language Learners. Philologica 2: 2, 299-311.

Deutsches Institut für Internationale Pädagogische Forschung (DIPF) (Hrsg.) (2006): Unterricht und Kompetenzerwerb in Deutsch und Englisch. Zentrale Befunde der Studie Deutsch-Englische-SchülerleistungenInternational (DESI). Frankfurt am Main: 61 ff [Online: www.dipf.de/ desi/DESI Zentrale Befunde.pdf 5.5.2010].

DESI-Konsortium (Hrsg.) (2008), Unterricht und Kompetenzerwerb in Deutsch und Englisch. DESI-Ergebnisse Band 2. Weinheim: Beltz Pädagogik.

Ehlers, Swantje (2014), Lesen im Kontext von Migration. IMIS-Beiträge: $45,53-66$.

Elsner, Daniela (2007), Hörverstehen im Englischunterricht der Grundschule. Ein Leistungsvergleich zwischen Kindern mit Deutsch als Muttersprache und Deutsch als Zweitsprache. Frankfurt am Main: Peter Lang.

Elsner, Daniela (2009), Englisch lernen als dritte Sprache: Was unterscheidet den zwei- oder mehrsprachigen vom einsprachigen Fremdsprachenlerner? Praxis Fremdsprachenunterricht 6: 2, 4-8.

Elsner, Daniela (2011), Raising plurilingual awareness in teacher education. In: Breidbach, Stephan; Elsner, Daniela \& Young, Andrea (Hrsg.) (2011), Language Awareness in Teacher Education. Frankfurt am Main: Peter Lang, 183-200. 
Elsner, Daniela (2012), Developing Multiliteracies, Plurilingual Awareness \& Critical Thinking in the Primary Language Classroom with Multilingual Virtual Talking Books. Encuentro 20: $16 \mathrm{ff}$.

Elsner, Daniela (2012), Muliliteracy Practices als Lernziel: Mehrsprachige Gedichte von Pat Mora und Antoine Cassar. In: Ahrens, Rüdiger; Eisenmann, Maria \& Hammer, Julia (Hrsg.) (2012), Anglophone Literaturdidaktik. Zukunftsperspektiven für den Englischunterricht. Heidelberg: Winter, 409-424.

Elsner, Daniela (2013), Muliliteracy Practices und Translanguaging - Zum Einsatz mehrsprachiger Gedichte im Englischunterricht der Sekundarstufe am Beispiel von Pat Mora und Antoine Cassar. In: Ahrens, Rüdiger; Eisenmann, Maria \& Hammer, Julia (Hrsg.) (2013), Literatur im Interkulturellen Kontext. Zukunftsperspektiven für den Englischunterricht. Heidelberg: Winter, $16 \mathrm{ff}$.

EVENING - Design, Durchführung, Ergebnisse. In: Engel, Gaby; GrootWilken, Bernd \& Thürmann, Eike (Hrsg) (2009), Englisch in der Primarstufe Chancen und Herausforderungen. Evaluation und Erfahrungen aus der Praxis. Berlin: Cornelsen, 124-215.

Franceschini, Rita (2002), Das Gehirn als Kulturinskription. In: MüllerLancé, J. \& Riehl, Claudia Maria (Hrsg.) (2002), Ein Kopf-viele Sprachen. Aachen: Shaker, $54 \mathrm{ff}$.

van Gelderen Amos; Schoonen, Rob; de Glopper, Kees; Hulstijn, Jan; Snellings, Patrick; Simis, Annegien \& Stevenson,. Marie (2003), Roles of linguistic knowledge, metacognitive knowledge and processing speed in L3, L2 and L1 reading comprehension. International Journal of Bilingualism 7: 1, 7-25.

Gibson, Martha \& Hufeisen, Britta (2007), Überlegungen zur Tertiärsprachenproblematik aus Sicht der Psycholinguistik und der Fehleranalyse. Zeitschrift für Fremdsprachenforschung 18: 1, 27-41.

Göbel, Kerstin; Rauch, Dominique \& Vieluf, Svenja (2011), Leistungsbedingungen und Leistungsergebnisse von Schülerinnen und Schülern türkischer, russischer und polnischer Herkunftssprachen. Zeitschrift für Interkulturellen Fremdsprachenunterricht 16: 2, 50-65.

Gogolin, Ingrid (2014), Integration durch Bildung. Mehrsprachigkeit fördern: Erkenntnisse und einige Ideen für die Praxis. Amtsblatt des Ministeriums für Schule und Weiterbildung NRW: 09, 415-417. 
Groot-Wilken, Bernd; Engel, Gaby \& Thürmann, Eike (2007), Listening and Reading Comprehension. Erste Ergebnisse einer Studie zu Englisch ab Klasse 3 an nordrhein-westfälischen Grundschule, Forum Schule 18: 1 [Online: http://partner-fuer-schule.nrw.de/fileadmin/user upload/forumschule/forum-schule-archiv/fs18/magein.html].

Hesse, Herman-Günter; Göbel, Kerstin \& Hartig, Johannes (2008), Sprachliche Kompetenzen von mehrsprachigen Jugendlichen und Jugendlichen nicht-deutscher Erstsprache. In: DESI-Konsortium; Klieme, Eckhard (Hrsg.) (2008), Unterricht und Kompetenzerwerb in Deutsch und Englisch. Ergebnisse der DESI Studie. Weinheim und Basel: Beltz, 208-230.

Hessisches Kultusministerium (Hrsg.) (2008), Lehrplan Englisch, Gymnasialer Bildungsgang, Jahrangsstufe 5-12. Wiesbaden.

$\mathrm{Hu}$, Adelheid (2010), Migrationsbedingte Mehrsprachigkeit und schulischer Fremdsprachenunterricht - revisited. In: Appel, Joachim; Doff, Sabine; Rymarczik, Jutta \& Thaler, Engelbert (Hrsg.) (2010), Foreign Language Teaching-History, Theory, Methods. Münchener Arbeiten zur Fremdsprachen-Forschung. München: Langenscheidt, 65-82.

Hufeisen, Britta (2003), L1, L2, L3, L4, Lx - alle gleich? Linguistische, lernerinterne und lernerexterne Faktoren in Modellen zum multiplen Spracherwerb. In: Baumgarten, Nicole; Böttger, Claudia; Motz, Markus \& Probst, Julia (Hrsg.) (2003), Übersetzen, Interkulturelle Kommunikation, Spracherwerb und Sprachvermittlung - das Leben mit mehreren Sprachen. Festschrifte für Juliane House zum 60. Geburtstag. Zeitschrift für Interkulturellen Fremdsprachenunterricht 8: 2/3, 97-109 [Online: www. ualberta.ca/ german/ejournal/Hufeisen1.htm, 97-109 15.12.2014].

Jessner, Ulrike (2008), Teaching third languages. Findings, trends and challenges. Language Teaching 41: 1, 15-56.

Köller, Olaf; Knigge, Michael \& Tesch, Bernd (Hrsg.) (2010), Sprachliche Kompetenzen im Ländervergleich. Befunde des ersten Ländervergleichs zur Überprüfung der Bildungsstandards für den Mittleren Schulabschluss in den Fächern Deutsch, Englisch und Französisch. Zusammenfassung. Münster: Waxmann.

Kollmeyer, Kathrin (2007), Englischunterricht als Fenster zur Mehrsprachigkeit. In: Elsner, Daniela; Küster, Lutz \& Viebrock, Britta (Hrsg.) (2007), Fremdsprachenkompetenzen für ein wachsendes Europa. Das Leitziel Multiliteralität. Frankfurt am Main: Peter Lang, 257-268. 
Landesinstitut für Schule - NRW (2014), [Online: http://www.schulentwick lung.nrw.de/lehrplaene/lehrplannavigator-s-i/realschule/englisch/kernlehr plan/aufgaben-ziele/aufgaben-und-ziele.html 12.12.2014].

Lohe, Viviane \& Elsner, Daniela (2015), Developing Language Awareness Primary School Children with Multilingual Virtual Talking Books: First Results of the Pilot Study. International Journal of Computer-Assisted Language Learning and Teaching 4: 4, 30-47.

Mora, Pat (1986), Unnatural Speech. In: Lauter, Paul (Hrsg.) (1994), The Heath Anthology of American Literature, 2. Lexington, MA: Heath and Company, 2989-2990.

Özdemir, Bengü (2006), Bilinguale Kinder im Englischunterricht. In: Pienemann, Manfred; Keßler, Jörg \& Roos, Eckart (Hrsg.) (2006), Englischerwerb in der Grundschule. Paderborn: Schöningh, 110-121.

Paulick, Christian \& Groot-Wilken, Bernd (2009), Rezeptive Fähigkeiten und Fertigkeiten am Ende der 4. Klasse unter besonderer Berücksichtigung der sprachlichen Schülerbiografien. In: Engel, Gaby; Groot-Wilken, Bernd \& Thürmann, Eike (Hrsg.) (2009), Englisch in der Primarstufe Chancen und Herausforderungen. Evaluation und Erfahrungen aus der Praxis. Berlin: Cornelsen Scriptor, 179-196.

Peltzer-Karpf, Annemarie (2003), Frühkindliche Erziehung zur Zweisprachigkeit. In: Bausch, Karl-Richard; Christ, Herbert \& Krumm, HansJürgen (Hrsg.) (2003), Handbuch Fremdsprachenunterricht (4. Aufl.). Tübingen: Francke, 445-449.

PISA-Konsortium Deutschland (Hrsg.) (2007), PISA 2006. Die Ergebnisse der dritten internationalen Vergleichsstudie. Münster: Waxmann.

PISA-Konsortium (Hrsg.) (2010), PISA 2009. Bilanz nach einem Jahrzehnt. Münster: Waxmann.

Poarch, Gregory (2013), Some thoughts on bilingualism. In: Elsner, Daniela \& Keßler, Jörg-U. (Hrsg.) (2013), Bilingual Education in Primary School. Tübingen: Narr Verlag, 7-15.

Portnaia, Natalia (2007), Englischlernen in der Grundschule aus der Sicht von Kindern mit migrationsbedingter Zwei-/ Mehrsprachigkeit in Deutschland. In: Elsner, Daniela; Küster, Lutz \& Viebrock, Britta. (Hrsg.) (2007), Fremdsprachenkompetenzen für ein wachsendes Europa. Das Leitziel Multiliteralität. Frankfurt am Main: Peter Lang, 107-120.

Riehl, Eva-Maria (2007), Das mehrsprachige Gehirn. Newsletter des Kompetenzzentrums für Sprachförderung 1: 30-32. 
Sanders, Marianne \& Meijers, Guust (2007), English as L3 in the elementary school. International Review for Applied Linguistics: 107-108, 59-78.

Schmid-Schönbein, Gisela (2009), Didaktik und Methodik für den Englischunterricht. Berlin: Cornelsen.

Singleton, David \& Aronin, Larissa (2007), Multiple Language Learning in the Light of the Theory of Affordances. Innovation in Language Learning and Teaching 1: 1, 83-96.

Snow, R.E. (1998), Abilities as aptitudes and achievements in learning situations. In: McArdle, J.J. \& Woodcock, R.W. (Hrsg.) (1998), Human Cognitive Abilities in Theory and Practice. Mahwah, NJ: Lawrence Erlbaum Associates, 93-112.

Weskamp, Ralf (2007), Mehrsprachigkeit. Sprachevolution, kognitive Sprachverarbeitung und schulischer Fremdsprachenerwerb. Braunschweig: Bildungshaus Schulbuchverlage.

Wildemann, Anja; Esteve, Olga; Hoodgarzadeh, Mahzad \& Walter, Rebecca (2014), Ein Beitrag zur Sensibilisierung für eine Mehrsprachigkeitsdidaktik in der Lehrerbildung. In: Abendroth-Timmer, Dagmar \& Henning, Eva-Maria (Hrsg.) (2014), Plurilingualism and Multiliteracies: International Research on Identity Construction in Language Education. KFU-Reihe. Peter Lang: Frankfurt am Main, 229-244.

Wilden, Eva \& Porsch, Raffaela (2015, erscheint): Die Hör- und Leseverstehensleistungen von Kindern im Fach Englisch am Ende der Grundschulzeit unter besonderer Berücksichtigung lebensweltlicher Ein- und Mehrsprachigkeit. In: Kötter, Markus \& Rymarczyk, Jutta (Hrsg.) (2014), Englischunterricht auf der Primarstufe: neue Forschungen - weitere Entwicklungen. Frankfurt am Main: Peter Lang. 


\section{Jim Cummins \\ Inclusion and Language Learning: Pedagogical Principles for Integrating Students from Marginalized Groups in the Mainstream Classroom}

In this paper, I analyze patterns of school achievement among students who come from communities that have been socially marginalized in the wider society and suggest evidence-based directions for increasing these students' educational success. Although each social context is unique, some generalizations regarding patterns of achievement and causes of underachievement can be made based on the research evidence. Identification of causal factors, in turn, enables us to highlight instructional interventions that respond to these causal factors. Initially, I elaborate on the meaning of the construct of 'inclusion' and also discuss the notion of 'socially marginalized groups' and who fits into this category.

\section{Definitions of 'Inclusion' and 'Social Marginalization'}

Although the construct of 'inclusion' emerged initially in the area of special needs education with the intent of reducing or eliminating the segregation from mainstream classrooms of students with learning difficulties or physical handicaps, the scope of the construct has expanded in recent years to emphasize the importance of providing equitable educational environments to all students. This emphasis is evident in the definition proposed by the United Nations Educational, Scientific, and Cultural Organization (UNESCO):

Inclusion is thus seen as a process of addressing and responding to the diversity of needs of all children, youth and adults through increasing participation in learning, cultures and communities, and reducing and eliminating exclusion within and from education. It involves changes and modifications in content, approaches, structures and strategies, with a common vision that covers all children of the appropriate age range and a conviction that it is the responsibility of the regular system to educate all children. (UNESCO 2009a: 8-9)

The construct of 'social marginalization' is defined by UNESCO (2009b) as "a form of acute and persistent disadvantage rooted in underlying social inequalities" (p. 1) and it includes (in many societies) "girls and women, 
hard-to-reach groups such as indigenous people and ethnic minorities, poor households, people living in informal settlements, individuals with disabilities, rural populations, nomadic populations those affected by armed conflict and HIV and AIDS, and street and working children" (p. 1). The focus in this paper is on a subset of these marginalized groups, specifically indigenous and ethnic minorities, migration-background communities, and students from low socioeconomic status (SES) backgrounds. The central question concerns the pedagogical directions implied by an inclusive education approach to addressing the educational needs of students from these marginalized groups. UNESCO $(2009 \mathrm{~b})$ provides a starting point for examining how an inclusive educational approach might respond to the acute and persistent disadvantage experienced by students from marginalized groups:

Learning environment matters. Typically those who enter school carrying the weight of disadvantage receive the worst education. They are often taught by poorly trained teachers, sometimes in a language they do not understand. Textbooks are frequently unavailable or include material that depicts negative stereotypes. Governments can address these problems by creating an enabling learning environment, beginning with providing incentives for skilled teachers to work in marginalized areas. Supporting intercultural and bilingual education can strengthen achievement among disadvantaged ethnic minorities. (UNESCO 2009b: 4-5)

\section{A Current Example of Educational and Social Marginalization}

Throughout the history of formal education, societal power relations have operated to exclude socially marginalized groups from educational opportunities. In the North American context, this process is evident in the experiences of groups such as African Americans, Latino/a students, and indigenous students. The link between societal power relations and the school experiences of some minority group students has been succinctly expressed by Ladson-Billings (1995: 485) with respect to African-American students: "The problem that African-American students face is the constant devaluation of their culture both in school and in the larger society."

This process continues in both overt and covert forms in many western societies. In the European context, discrimination against Roma students is perhaps the most blatant. A recent Amnesty International (2015a) report on the exclusion of Roma students from meaningful education in the Czech 
Republic found that systematic discrimination is the norm. The press release associated with this report expressed this reality in blunt terms:

Amnesty International found that Romani children are routinely placed into schools for pupils with 'mild mental disabilities' with reduced learning possibilities.

Nearly a third of pupils in these so-called 'practical schools' are Roma, despite the Romani community making up less than $3 \%$ of the Czech Republic. [...] Romani children also suffer from segregation in mainstream education, often ending up either in Roma-only schools or within mixed schools in a separate building or classroom. (Amnesty International 2015b)

An article in the Toronto Star quoted Salil Shetty, Amnesty International's secretary general, as follows: "The widespread segregation of Romani children is a horrifying example of systematic prejudice, with schools introducing children to bitter discrimination at an early age. [...] Let's call this what it is: racism, pure and simple" (Associated Press 2015).

The experience of most migration-background communities in Europe and North America is, for the most part, very different from the overt discrimination and exclusion experienced by Roma communities. In most countries, national policies of equality of educational opportunity attempt to ensure that all children, regardless of socioeconomic, linguistic, religious or cultural background have access to schooling. However, despite the rhetoric of equality and inclusion, it is legitimate to inquire to what extent this societal commitment to equity is realized in practice. Certainly, inequality in educational outcomes exists for low-SES, migration-background, and other socially marginalized students. In the following section, the extent of inequality of educational outcomes for migration-background students is summarized, as revealed by the Organisation for Economic Cooperation and Development's (OECD) Programme for International Student Achievement (PISA) studies. Then the causal factors underlying inequality of educational outcomes are examined and evidence-based responses to these causal factors designed to promote inclusion and equity of outcomes are proposed.

\section{Inequality of Outcomes among Migration-Background Students}

Three potential sources of educational disadvantage characterize the social situation of many migration-background communities: (a) home-school 
language switch requiring students to learn academic content through a second language; (b) low-SES associated with low family income and/or low levels of parental education; (c) marginalized group status deriving from social discrimination and/or racism in the wider society. Some communities in different countries are characterized by all three risk factors (e.g., many Spanish-speaking students in the United States, many Turkish-speaking students in different European countries). In other cases, only one risk factor may be operating (e.g., middle-class African-American students in the United States, middle-class Romanian-speaking students attending school in Germany). Although these three social conditions constitute risk factors for students' academic success, they become realized as educational disadvantage only when the school fails to respond appropriately or reinforces the negative impact of the broader social factors. For example, as documented above, the social discrimination that Roma students experience throughout Europe has been educationally reinforced in some countries by educators who label them as intellectually handicapped and place them in segregated classes for intellectually handicapped students.

The reading performance of 15 -year-old first- and second-generation immigrant-background students from several countries on the OECD's PISA project is shown in Table 1. Students tend to perform better in countries such as Canada and Australia that have encouraged immigration during the past 40 years and that have a coherent infrastructure designed to integrate immigrants into the society (e.g. free adult language classes, language support services for students in schools, rapid qualification for full citizenship, etc.). Additionally, both Canada and Australia have explicitly endorsed multicultural philosophies at the national level aimed at promoting respect across communities and expediting the integration of newcomers into the broader society. In Canada (2003 assessment) and Australia (2006 assessment), second-generation students (born in the host country) performed slightly better academically than native speakers of the school language. Some of the positive results for Australia and Canada can be attributed to selective immigration that favours immigrants with strong educational qualifications. In both countries, the educational attainments of adult immigrants are as high, on average, as those of the general population. 
Table 1: PISA Reading Scores 2003 and 2006 (based on data presented in Christensen and Steglitz 2008; Gen 1 = first generation students born outside the host country, Gen 2 = second generation students born in the host country; negative scores indicate performance below country mean, positive scores indicate performance above country mean; overall mean is 500)

\begin{tabular}{|l|c|c|c|c|}
\hline & $\begin{array}{c}\text { PISA 2003 } \\
\text { Gen 1 }\end{array}$ & $\begin{array}{c}\text { PISA 2003 } \\
\text { Gen 2 }\end{array}$ & $\begin{array}{c}\text { PISA 2006 } \\
\text { Gen 1 }\end{array}$ & $\begin{array}{c}\text { PISA 2006 } \\
\text { Gen 2 }\end{array}$ \\
\hline Australia & -12 & -4 & +1 & +7 \\
\hline Austria & -77 & -73 & -48 & -79 \\
\hline Belgium & -117 & -84 & -102 & -81 \\
\hline Canada & -19 & +10 & -19 & 0 \\
\hline Denmark & -42 & -57 & -79 & -64 \\
\hline France & -79 & -48 & -45 & -36 \\
\hline Germany & -86 & -96 & -70 & -83 \\
\hline Netherlands & -61 & -50 & -65 & -61 \\
\hline Norway & -68 & -59 & -63 & -42 \\
\hline Sweden & -89 & -20 & -68 & -29 \\
\hline Switzerland & -93 & -53 & -85 & -48 \\
\hline United Kingdom & & & -44 & -7 \\
\hline United States & -50 & -22 & & \\
\hline
\end{tabular}

By contrast, second generation students tend to perform very poorly in countries that have been characterized by highly negative attitudes towards immigrants (e.g., Austria, Belgium, Denmark, Germany). The overall picture for many European countries that emerges from the PISA data is that second generation students who have experienced all their socialization in the host country do not perform much better than first generation students who may have had significantly less exposure to the host country language and culture. Clearly, despite full access to state-provided educational opportunities, many immigrant-background students are not succeeding academically.

\section{Potential Causal Factors and Evidence-Based Educational Responses}

In addition to documenting patterns of school achievement among migrationbackground students, the PISA studies have also identified the potentially causal role of several variables. For example, the OECD (2010a) reports 
that the SES of individual students exerted a highly significant effect on achievement in the PISA studies: "On average across OECD countries, 14\% of the differences in student reading performance within each country is associated with differences in students' socio-economic background" (OECD 2010a: 14). However, this report noted that the effect of the school's economic, social and cultural status on students' performance is much stronger than the effects of the individual student's socio-economic background. In other words, when students from low-SES backgrounds attend schools with a socio-economically advantaged intake, they tend to perform significantly better than when they attend schools with a socio-economically disadvantaged intake. This difference between the SES of individual students and the collective SES of students within particular schools highlights the effects of housing (and consequent educational) segregation on patterns of school achievement.

Another important finding that has emerged from several of the PISA studies concerns the role of reading engagement in determining reading achievement among 15-year olds. The 2000 PISA study (OECD 2004) reported that the level of a student's reading engagement was a better predictor of reading performance than his or her SES. In more recent PISA studies, the OECD (2010b) reported that approximately one-third of the association between reading performance and students' SES was mediated by reading engagement. The implication is that schools can potentially 'push back' about one-third of the negative effects of socioeconomic disadvantage by ensuring that students have access to a rich print environment and become actively engaged with literacy. The credibility of this inference is supported by considerable data showing that many low-SES students experience limited access to print in their homes, neighborhoods and schools (Duke 2000; Neuman \& Celano 2001). The causal link between print access/literacy engagement and reading attainment has been demonstrated in numerous research studies (e.g., Elley \& Mangubhai 1983; Lindsay 2010; Mol \& Bus 2011; Sullivan \& Brown 2013).

Table 2 elaborates on the potential educational disadvantages associated with a home-school language switch, low-SES, and marginalized group status and also specifies the evidence-based educational responses that are likely to have the highest impact in addressing these sources of potential disadvantage. 
Table 2: High-Impact Instructional Responses to Sources of Potential Academic Disadvantage

\begin{tabular}{|c|c|c|c|}
\hline $\begin{array}{l}\text { Student } \\
\text { Background }\end{array}$ & Linguistically Diverse & Low-SES & $\begin{array}{l}\text { Marginalized } \\
\text { Status }\end{array}$ \\
\hline $\begin{array}{l}\text { Sources of } \\
\text { potential } \\
\text { disadvantage }\end{array}$ & $\begin{array}{l}\text { - Failure to understand } \\
\text { instruction due to } \\
\text { home-school language } \\
\text { differences; }\end{array}$ & $\begin{array}{l}\text { - Inadequate } \\
\text { healthcare and/or } \\
\text { nutrition; } \\
\text { - Housing } \\
\text { segregation; } \\
\text { - Lack of cultural and } \\
\text { material resources } \\
\text { in the home due to } \\
\text { poverty; } \\
\text { - Inadequate access to } \\
\text { print in home and } \\
\text { school }\end{array}$ & $\begin{array}{l}- \text { - Societal } \\
\text { discrimination; } \\
\text { - Low teacher } \\
\text { expectations; } \\
\text { - Stereotype } \\
\text { threat; } \\
\\
\text { - Identity } \\
\text { devaluation }\end{array}$ \\
\hline $\begin{array}{l}\text { Evidence- } \\
\text { based } \\
\text { instructional } \\
\text { response }\end{array}$ & $\begin{array}{l}\text { - Scaffold } \\
\text { comprehension } \\
\text { and production of } \\
\text { language across the } \\
\text { curriculum; } \\
\text { - Engage students' } \\
\text { multilingual } \\
\text { repertoires; } \\
\text { - Reinforce academic } \\
\text { language across the } \\
\text { curriculum }\end{array}$ & $\begin{array}{l}\text { - Maximize print } \\
\text { access and literacy } \\
\text { engagement } \\
\text { - Reinforce academic } \\
\text { language across the } \\
\text { curriculum }\end{array}$ & $\begin{array}{l}\text { - Connect } \\
\text { instruction to } \\
\text { students' lives; } \\
\\
\text { - Affirm student } \\
\text { identities in } \\
\text { association } \\
\text { with literacy } \\
\text { engagement }\end{array}$ \\
\hline
\end{tabular}

\section{Home-School Language Differences}

Two issues are relevant to the interpretation of data relating to the effects of a home-school language switch: (a) To what extent does the simple fact of speaking a language other than the school language (L2) at home constitute a cause of underachievement? (b) What instructional programs or initiatives are most effective in helping students learn the L2?

Home language use and achievement. The PISA data (OECD 2012; Stanat \& Christensen 2006), at first sight, appear to show a negative relationship between language spoken at home and academic achievement. In both mathematics and reading, first and second generation immigrantbackground students who spoke their L1 at home were significantly behind their peers who spoke the school language at home. Christensen and Stanat 
(2007) conclude: "These large differences in performance suggest that students have insufficient opportunities to learn the language of instruction" (p. 3). German sociologist Hartmut Esser (2006) similarly argues on the basis of PISA data that "the use of the native language in the family context has a (clearly) negative effect" (p. 64). He further argues that retention of the home language by immigrant children will reduce both motivation and success in learning the host country language (2006: 34).

These interpretations of the data do not stand up to critical scrutiny. In the first place, the PISA authors (OECD 2012; Stanat \& Christensen 2006) consistently interpret the relationships observed between home use of L1 and school achievement in causal terms despite the fact that the relationships observed are correlational rather than causal. In order to infer causality, contradictory data would have to be accounted for and the unique variance associated with language spoken at home would have to be identified and isolated from other mediating variables. Neither of these conditions is met in the PISA reports. Specifically, no relationship was found between home language use and achievement in the two countries where immigrant students were most successful (Australia and Canada) and the relationship disappeared for a large majority (10 out of 14) of OECD-member countries when SES and other background variables were controlled (Stanat \& Christensen 2006, Table 3.5: 200-202). The disappearance of the relationship in a large majority of countries suggests that language spoken at home does not exert any independent effect on achievement but is rather a proxy for variables such as socioeconomic status and length of residence in the host country.

Beyond the PISA data, the argument that L1 use at home will exert a negative effect on achievement in L2 is refuted by the academic success of vast numbers of bilingual and multilingual students in countries around the world. Thus, parents who interact consistently with their children in L1 as a means of promoting bilingualism and biliteracy can do so with no concern that this will impede their children's acquisition of the school language.

Effective instructional responses. The international research data strongly supports the effectiveness of bilingual education for minority group students (e.g., Gogolin 2005). Several recent comprehensive research reviews on bilingual education for underachieving minority language students suggest that in contexts where bilingual education is feasible (e.g., high concentration 
of particular groups), it represents a superior option to immersion in the language of the host country. In the North American context, for example, Francis, Lesaux and August (2006) report: "The meta-analytic results clearly suggest a positive effect for bilingual instruction that is moderate in size" (p. 397). Similarly, Lindholm-Leary and Borsato (2006) conclude that minority student achievement "is positively related to sustained instruction through the student's first language" (p. 201). Thus, bilingual education represents a legitimate and, in many cases, feasible option for educating immigrant and minority language students.

In cases where bilingual education is not feasible or is excluded from consideration for ideological reasons, instruction that engages students' multilingual repertories represents an effective tool for teachers to scaffold meaning, connect to students' lives, affirm their identities, and enhance awareness of how academic language works (Celic \& Selzer 2011). For example, newcomer students could write initially in their L1 and then work with peers, community volunteers, or technology programs such as Google Translate to translate their L1 writing to the school language. Google Translate will typically not provide a satisfactory translation by itself but it is usually sufficient to enable the teacher or peers to figure out what the newcomer student is trying to express and to work with him or her to come up with an accurate translation (Cummins 2001). Encouraging students to use their L1 as a cognitive tool is a form of scaffolding, a term commonly used to describe the temporary supports that teachers provide to enable learners to carry out academic tasks. These supports can be reduced gradually as the learner gains more expertise. They include strategies such as use of visuals and concrete experiences and demonstrations to increase comprehension.

Finally, there is a large degree of consensus among researchers and policymakers that academic language is very different than the language we use in everyday conversational contexts. For example, academic language is characterized by low-frequency vocabulary and grammatical expressions (e.g., passive voice) that are seldom used in face-to-face conversational contexts. These differences represent a major reason why it typically takes considerably longer for immigrant students to catch up to their native-speaking peers in academic language (at least 5 years and sometimes considerably longer) than in conversational language (Collier 1987; Cummins 1981). The chal- 
lenges newcomer students face in catching up academically imply that all teachers (not just language specialists) should know how to support students in acquiring academic skills in the school language.

\section{Students from Low-SES Backgrounds}

Christensen and Segeritz (2008) note that the impact of SES on achievement varies widely among countries. For example, Australia, Canada, and the United Kingdom exhibit high levels of student achievement in Science and a lower-than-average association between SES and Science performance. Norway also showed a low level of association between SES and Science ( $<10 \%$ variance explained), but overall performance was below average. These results show that despite the strong overall relationship between SES and academic performance, some countries do succeed in promoting both equity (low-SES students perform relatively well) and excellence (overall performance is strong). In fact, according to the OECD (2010a), the "best performing school systems manage to provide high-quality education to all students...regardless of their own background or the school they attend" (p. 13).

Countries also vary in the extent to which they provide universal healthcare and other social supports. Thus, in Table 2, the impact of variables such as inadequate healthcare and/or nutrition is likely to be greater in the United States context than in most European contexts for the simple reason that until recently many families living in poverty had very limited access to healthcare, including pre-natal care for pregnant mothers.

Effective instructional responses. Some of the sources of potential educational disadvantage associated with SES are beyond the capacity of individual schools to address (e.g., housing segregation) but the potential negative effects of other factors can be ameliorated by school policies and instructional practices. In this regard, the two sources of potential disadvantage that are most significant are the limited access to print that many low-SES students experience in their homes, neighborhoods and schools (Duke 2000; Neuman \& Celano 2001) and the more limited range of language interaction that children may experience in some low-SES families as compared to more affluent families. For example, Hart and Risley (1995) in the United States documented large differences in the quantity of 
language interaction experienced by children whose mothers were on social assistance (i.e., living in poverty) in comparison to middle-class children. Caution should obviously be exercised in generalizing this finding to all children living in poverty. The logical inference that derives from these differences is that schools serving low-SES students should (a) immerse them in a print-rich environment in order to promote literacy engagement across the curriculum and (b) focus in a sustained way on how academic language works and enable students to take ownership of academic language by using it for powerful (i.e., identity-affirming) purposes.

Guthrie (2004) notes that the construct of literacy engagement incorporates notions of time on task (reading extensively), affect (enthusiasm and enjoyment of literacy), depth of cognitive processing (strategies to deepen comprehension), and active pursuit of literacy activities (amount and diversity of literacy practices in and out of school). He points out that engaged readers are active and energized in reading and use their minds with an emphasis on either cognitive strategies or conceptual knowledge. Furthermore, he notes that engaged reading is often socially interactive insofar as engaged students are capable of discussion or sharing with friends despite the fact that much of their reading may be solitary. He cited the PISA data as showing that students

whose family background was characterized by low income and low education, but who were highly engaged readers, substantially outscored students who came from backgrounds with higher education and higher income, but who themselves were less engaged readers. Based on a massive sample, this finding suggests the stunning conclusion that engaged reading can overcome traditional barriers to reading achievement, including gender, parental education, and income. (p. 5)

\section{Students from Marginalized Communities}

As noted in an earlier section, there is extensive research documenting the chronic underachievement of groups that have experienced systematic long-term discrimination in the wider society. This constant devaluation of culture is illustrated in the well-documented phenomenon of stereotype threat (Steele 1997). Stereotype threat refers to the deterioration of individuals' task performance in contexts where negative stereotypes about their social group are communicated to them. Schofield and Bangs (2006) summarized the implications of this phenomenon for marginalized group students: "stereotype threat [...] can seriously undercut the achievement of 
immigrant and minority students [...] undermine the academic performance of children as young as 5 or 6 years of age, and its effects can be quite large" (p. 93). Thus, there is a clear link between societal power relations, identity negotiation, and task performance.

Among linguistically diverse students, the home language represents a very obvious marker of difference from dominant groups. Despite increasing evidence of the benefits of bilingualism for students' cognitive and academic growth and future economic success (e.g. Agirdag 2014; Barac \& Bialystok 2011), schools in many contexts continue to prohibit students from using their L1 within the school, thereby communicating to students the inferior status of their home languages and devaluing the identities of speakers of these languages. This pattern is illustrated in a study of Turkishbackground students in Flemish secondary schools carried out by Agirdag (2010). He concluded:

[O]ur data show that Dutch monolingualism is strongly imposed in three different ways: teachers and school staff strongly encourage the exclusive use of Dutch, bilingual students are formally punished for speaking their mother tongue, and their home languages are excluded from the cultural repertoire of the school. At the same time, prestigious languages such as English and French are highly valued. (p. 317)

Effective instructional responses. How can schools counteract the negative effects of societal power relations that devalue minority group identities? Ladson-Billings (1994) expressed the essence of an effective instructional response: "When students are treated as competent they are likely to demonstrate competence" (1994: 123). In other words, educators, both individually and collectively, must challenge the devaluation of students' language, culture, and identity in the wider society by implementing instructional strategies that enable students to develop 'identities of competence' (Manyak 2004) in the school context. These instructional strategies will communicate high expectations to students regarding their ability to succeed academically and support them in meeting these academic demands by affirming their identities and connecting curriculum to their lives (see Cummins \& Early 2011; Hélot, Sneddon \& Daly 2015).

Among the overlapping instructional strategies reviewed by Cummins and Early (2015) that have been successfully implemented for affirming students' identities are (a) encouraging immigrant-background and socially 
marginalized students to use their L1 as a cognitive tool for carrying out academic tasks; (b) promoting opportunities for students to develop literacy skills in their home languages; (c) enabling students to write and webpublish literary and multimodal creative work (e.g., stories, poems, videos, music); this work can be in the school language or (ideally) in multiple languages depending on the context and language skills of the students; and (d) implementing projects focused on inquiry and knowledge generation that encourage students to use both their L1 and L2, perhaps in partnership with a collaborating class in another location. These forms of pedagogy are aimed at enabling students to use language for powerful identity-affirming purposes and motivating them to engage academically.

\section{Implications for Inclusion}

The instructional implications of the analysis of potential sources of educational disadvantage and evidence-based educational responses (Table 2) has highlighted the fact that underachievement among immigrant-background students is not caused by home use of a language other than the school language. L1 use at home represents a potential source of educational disadvantage only when the school fails to provide appropriate support to enable students to develop academic skills in the school language. Furthermore, underachievement is observed predominantly among linguistically diverse students who are also experiencing the effects of low-SES and/or marginalized group status in the host country. Thus, instruction must also address the sources of potential disadvantage that characterize low-SES and marginalized group students. This will include maximizing students' engagement with literacy (ideally in both L1 and L2) and enabling them to use language powerfully in ways that enhance their academic and personal self-concept. The following instructional strategies designed to promote inclusion and equity of educational outcomes emerge from this analysis:

- Scaffold comprehension and production of language;

- Engage students' multilingual repertoires;

- Reinforce academic language across the curriculum;

- Maximize print access and literacy engagement;

- Connect instruction to students' lives;

- Affirm students' identities in association with literacy engagement. 
How can schools generate policies and practices that will promote these instructional goals? Planned change in educational systems always involves choice. Administrators and policy-makers make choices at a broad system level, school principals make choices at the level of individual schools, and teachers make choices within their classrooms. Thus, individual educators always have the power to exercise agency-they are never powerless, although they frequently work in conditions that may constrain their creativity and agency. While they rarely have complete freedom, educators determine for themselves the social and educational goals they want to achieve with their students. They always have options with respect to their orientation to students' language and culture, the forms of parent and community participation they encourage, and in the ways they implement pedagogy and assessment (Cummins 2001).

The process of articulating pedagogical choices and engaging in a collaborative pedagogical inquiry at the level of the school might start with discussion of what image of the child is embedded in current instructional policies and practices. Among the questions that educators might explore are those in Table 3.

Table 3: Collaborative inquiry about the image of the child embedded in instruction

What image of the child are we sketching in our instruction:

- Capable of becoming bilingual and biliterate?

- Capable of higher-order thinking and intellectual accomplishments?

- Capable of creative and imaginative thinking?

- Capable of creating literature and art?

- Capable of generating new knowledge?

- Capable of thinking about and finding solutions to social issues?

A similar collaborative inquiry process can be instituted for the other instructional directions articulated above. The kinds of questions that might be pursued are illustrated with respect to literacy engagement (Table 4) and identity affirmation (Table 5). Examples from one highly diverse elementary school in the Toronto area are shown in Figures 1 and 2. Table 6 presents a Collaborative Inquiry template designed (a) to guide teachers and other educators in articulating the pedagogical choices that they are currently making in their classrooms and schools and (b) to consider alternative choices that might increase student engagement and instructional 
effectiveness. The categories in the template are flexible, and thus teachers in any particular school can modify the issues for discussion according to their particular circumstances and priorities.

Table 4: Collaborative pedagogical inquiry: Literacy engagement

To what extent are students immersed in a literacy-rich environment in primary and secondary school?

- Are they listening to and dramatizing stories from the earliest days of schooling?

- Do they have access to a well-stocked classroom library and the opportunity to borrow books to take home to read either individually or with their parents?

- Does the school library have books in the multiple languages represented by students in the school and/or dual language books?

- Does the school library encourage parents to come in and check out books with their children (e.g., by staying open for some time after regular school hours to accommodate parents' schedules)?

- Do students have opportunities within the classroom to discuss books they are reading?

- Is technology being used in creative ways? For example, are students uploading group or individual book reviews to appropriate web sites? Are they videotaping dramatized scenes or adaptations from books they have read?

- Has the school forged connections with the local public library to explore ways of promoting literacy engagement?

\section{Table 5: Collaborative pedagogical inquiry: Identity affirmation}

To what extent is the school enabling students to connect academic work to their own developing identities with the result that students develop a sense of pride in their linguistic talents and intellectual and literary accomplishments?

- To what extent do students and parents see signs and student work in multiple languages displayed at the school entrance and other public spaces (e.g., corridors) throughout the school?

- To what extent are newcomer students encouraged to use their L1s for completion of academic work and creative writing?

- To what extent are students' dual language books or projects displayed publicly (e.g., on a school web site) and showcased in a positive manner (e.g., on parents' nights etc.)?

- To what extent are students enabled to engage in partner class projects with multilingual speakers from other countries or regions using multiple languages to carry out collaborative projects?

- To what extent are students encouraged to compare their L1 with the school language in order to develop greater language awareness? 
Figure 1: Examples of multilingual signs and library books in Crescent Town elementary school in Toronto

\section{Creating an Identity-Affirming School Environment}

Validating Home Language and Culture
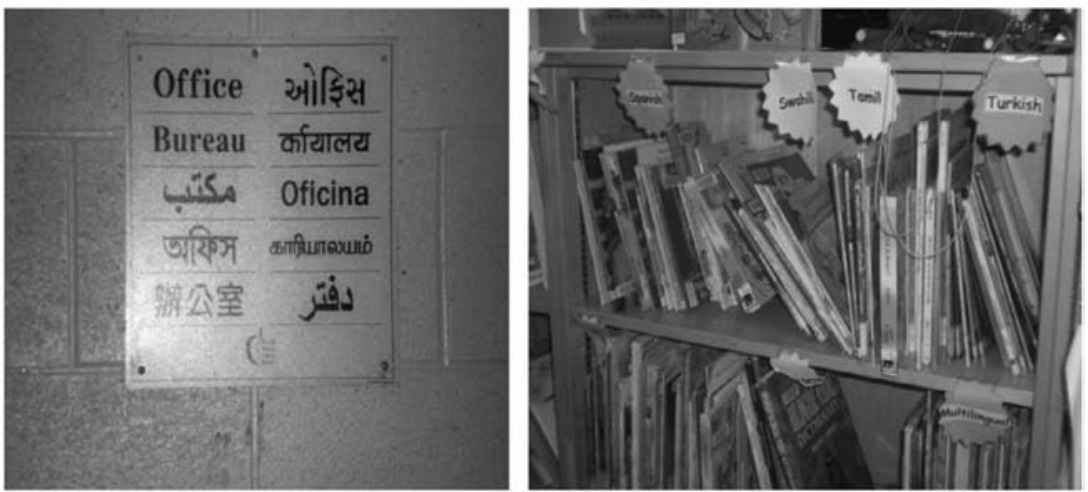

Figure 2: Showcasing students' intellectual inquiry projects in the school library (Crescent Town elementary school)

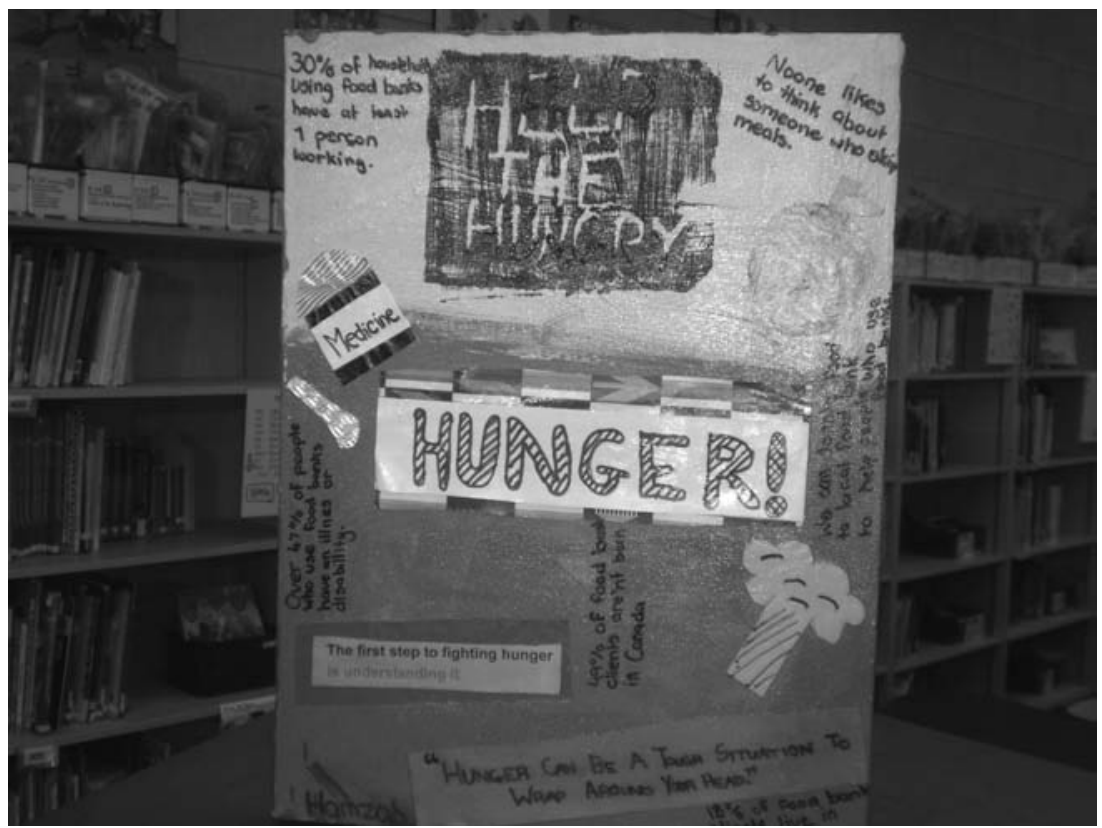

Christiane M. Bongartz and Andreas Rohde - 978-3-653-97188-0 Downloaded from PubFactory at 01/11/2019 10:41:16AM 
Table 6: School-based language and instructional planning

\begin{tabular}{|c|c|c|c|}
\hline \multicolumn{4}{|l|}{$\begin{array}{l}\text { School-based Language and Instructional Planning: } \\
\text { Articulating Choices and Taking Collective Action }\end{array}$} \\
\hline Instructional Options & \begin{tabular}{|l|} 
Current \\
Realities \\
Where Are \\
We?
\end{tabular} & $\begin{array}{l}\text { Vision for } \\
\text { the Future } \\
\text { Where Do } \\
\text { We Want } \\
\text { To Be? }\end{array}$ & $\begin{array}{l}\text { Getting it } \\
\text { Done } \\
\text { How Do } \\
\text { We Get } \\
\text { There? }\end{array}$ \\
\hline \multicolumn{4}{|l|}{$\begin{array}{l}\text { Literacy Engagement } \\
\text { How can we maximize students' interests in } \\
\text { and engagement with reading, writing, and } \\
\text { other forms of cultural expression? }\end{array}$} \\
\hline \multicolumn{4}{|l|}{$\begin{array}{l}\text { Content } \\
\text { How do we adapt curriculum materials to } \\
\text { link with students' existing knowledge and } \\
\text { cultural background? }\end{array}$} \\
\hline \multicolumn{4}{|l|}{$\begin{array}{l}\text { Cognition } \\
\text { How can we modify instruction to evoke } \\
\text { higher-order thinking among low-income and } \\
\text { culturally/linguistically diverse students? }\end{array}$} \\
\hline \multicolumn{4}{|l|}{$\begin{array}{l}\text { Tools } \\
\text { How can we use tools such as computers, } \\
\text { digitalcameras, camcorders, web pages, } \\
\text { etc. to enhance academic engagement and } \\
\text { achievement? }\end{array}$} \\
\hline \multicolumn{4}{|l|}{$\begin{array}{l}\text { Assessment } \\
\text { How can we assess students' academic work } \\
\text { in such a way that students themselves, } \\
\text { parents, and administrators gain a clear } \\
\text { picture of students' academic progress? }\end{array}$} \\
\hline \multicolumn{4}{|l|}{$\begin{array}{l}\text { Language/ Culture } \\
\text { What messages are we giving students about } \\
\text { the value of their languages and cultures? }\end{array}$} \\
\hline $\begin{array}{l}\text { Parental Involvement } \\
\text { How can we engage parents more effectively } \\
\text { as co-educators in such a way that their } \\
\text { cultural knowledge and experience can } \\
\text { support their children's academic progress? }\end{array}$ & & & \\
\hline
\end{tabular}




\section{Conclusion}

Although policy-makers and educators in most countries accept that inclusion and equity are desirable goals in education, the PISA findings with respect to the academic performance of migration-background students suggest that these goals are far from being achieved. The argument in this paper is that a major reason for the disappointing educational outcomes documented by PISA is that research evidence on the causes of underachievement has not been interpreted appropriately and, as a result, ineffective and largely evidence-free policies have been implemented. For example, policy-makers in many countries have chosen to ignore extensive empirical evidence suggesting the following: (a) factors associated with SES and broader patterns of societal power relations exert a major influence on educational outcomes; (b) literacy engagement is among the strongest predictors of reading performance and low-SES students have significantly less access to books and print than do higher-SES students; (c) students will engage academically only to the extent that classroom interactions and academic effort are identity-affirming.

The analysis in the present paper argues that school polices need to maximize print access and literacy engagement among low-SES and other marginalized group students. In addition, policies need to take account of the devaluation of student and community identities in both schools and the wider society and focus on enabling students to develop identities of competence (Manyak 2004) in association with academic engagement. In other words, schools focused on inclusion need to enable students to use language and literacy in ways that will affirm their identities and challenge the deficit orientation that is frequently built into programs and curricula for low-SES and bilingual learners of migration backgrounds.

\section{References}

Agirdag, Orhan (2010), Exploring bilingualism in a monolingual school system: Insights from Turkish and native students from Belgian schools. British Journal of Sociology of Education 31: 3, 307-321. DOI: 10.1080/01425691003700540.

Agirdag, Orhan (2014), The long-term effects of bilingualism on children of immigration: Student bilingualism and future earnings. International 
Journal of Bilingual Education and Bilingualism 17: 4, 449-464. [Online: http://dx.doi.org/10.1080/13670050.2013.816264] (accessed May 2015).

Amnesty International (2015a), Must try harder: Ethnic discrimination of Romani children in Czech schools. London: Author. [Online: http://www. amnestyusa.org/sites/default/files/musttryharder embargoed report.pdf] (accessed May 2015).

Amnesty International (2015b), Czech Republic: Systematic discrimination against Romani children in schools. [Online: https://www.amnesty. org/en/latest/news/2015/04/czech-republic-systematic-discriminationagainst-romani-children-in-schools/] (accessed September 2015).

Associated Press (2015, April 23), Czech Republic violating EU order on Roma: Amnesty International. Toronto Star. [Online: http://www.thestar. com/news/world/2015/04/23/czechrepublicviolatingeuorderonromaamnestyinternational.html] (accessed May 2015).

Barac, Raluca \& Bialystok, Ellen (2011), Cognitive development of bilingual children. Language Teaching 44: 1, 36-54.

Celic, Christina \& Seltzer, Kate (2011), Translanguaging: A CUNY-NYSIEB guide for educators. New York, NY: CUNY-NYSIEB. [Online: http:// www.nysieb.ws.gc.cuny.edu/files/2012/06/FINAL-TranslanguagingGuide-With-Cover-1.pdf (accessed May 2015).

Christensen, Gayle \& Stanat, Petra (2007, September), Language policies and practices for helping immigrant second-generation students succeed. The Transatlantic Task Force on Immigration and Integration convened by the Migration Policy Institute and Bertlesmann Stiftung. [Online: http://www. migrationinformation.org/transatlantic/l (accessed May 2015).

Christensen, Gayle \& Segeritz, Michael (2008), An international perspective on student achievement. In: Bertelsmann Stiftung (ed.), Immigrant students can succeed: Lessons from around the globe. Gütersloh: Verlag Bertelsmann Stiftung, 11-33.

Collier, Virginia P. (1987), Age and rate of acquisition of second language for academic purposes. TESOL Quarterly 21, 617-641.

Cummins, Jim (2001), Negotiating identities: Education for empowerment in a diverse society ( $2^{\text {nd }}$ ed.). Los Angeles: California Association for Bilingual Education.

Cummins, Jim \& Early, Margaret (eds.) (2011), Identity texts: The collaborative creation of power in multilingual schools. Stoke-on-Trent, England: Trentham Books. 
Cummins, Jim \& Early, Margaret (2015), Big ideas for expanding minds: Teaching English language learners across the curriculum. Toronto: Rubicon Press/Pearson Canada.

Duke, Nell K. (2000), For the rich it's richer: Print experiences and environments offered to children in very low and very high-socioeconomic status first-grade classrooms. American Educational Research Journal 37, 441-478. doi: 10.3102/00028312037002441.

Elley, Warwick B. \& Mangubhai, Francis (1983), The impact of reading on second language learning. Reading Research Quarterly 19, 53-67.

Esser, Hartmut (2006), Migration, language, and integration. AKI Research Review 4. Berlin: Programme on Intercultural Conflicts and Societal Integration (AKI), Social Science Research Center. [Online: http.//www. wzb.eu/zkd/aki/files/aki_research_review_4.] (accessed May 2015).

Francis, David J.; Lesaux, Nonie K. \& August, Diane (2006), Language of instruction. In: August, Diane \& Shanahan, Timothy (eds.), Developing literacy in second-language learners. Report of the National Literacy Panel on Language-Minority Children and Youth. Mahwah, NJ: Lawrence Erlbaum Associates Publishers, 365-413.

Genesee, Fred; Lindholm-Leary, Kathryn; Saunders, William M. \& Christian, Donna (eds.) (2006), Educating English language learners: A synthesis of research evidence. New York: Cambridge University Press.

Gogolin, Ingrid (2005), Bilingual education: The German experience and debate. In: Söhn, Janina (ed.), The effectiveness of bilingual school programs for immigrant children. AKI Research Review 2. Berlin: Programme on Intercultural Conflicts and Societal Integration (AKI), Social Science Research Center, 133-145. [Online: http://www.wzb.eu/zkd/aki/ files/aki_bilingual_school_programs.pdf] (accessed May 2015).

Guthrie, John T. (2004), Teaching for literacy engagement. Journal of Literacy Research 36, 1-30. doi: 10.1207/s15548430jlr3601_2.

Hart, Betty \& Risley, Todd R. (1995), Meaningful differences in the everyday experience of young American children. Baltimore, MD: Paul H. Brookes Publishing.

Hélot, Christine; Sneddon, Raymonde \& Daly, Nicola (eds.) (2014), Children's literature in multilingual classrooms: From multiliteracy to multimodality. London: IOE Press.

Ladson-Billings, Gloria (1994), The dreamkeepers: Successful teachers of African American children. San Francisco: Jossey-Bass Publishers. 
Ladson-Billings, Gloria (1995), Toward a theory of culturally relevant pedagogy. American Educational Research Journal 32, 465-91. doi: 10.3102/00028312032003465.

Lindholm-Leary, Kathryn \& Borsato, Graciela (2006), Academic achievement. In: Genesee, Fred; Lindholm-Leary, Kathryn; Saunders, William M. \& Christian, Donna (eds.), Educating English Language Learners. New York: Cambridge University Press, 176-222.

Lindsay, Jim (2010), Children's access to print material and educationrelated outcomes: Findings from a meta-analytic review. Naperville, IL: Learning Point Associates.

Manyak, Patrick C. (2004), "What did she say?" Translation in a primarygrade English immersion class. Multicultural Perspectives 6, 12-18. doi:10.1207/S15327892mcp0601_3.

Mol, Suzanne E., \& Bus, Adriana (2011), To read or not to read: A metaanalysis of print exposure from infancy to early adulthood. Psychological Bulletin 137: 2, 267-296. doi: 10.1037/a0021890.

Neuman, Susan B. \& Celano, Donna (2001), Access to print in low-income and middle-income communities: An ecological study of four neighbourhoods. Reading Research Quarterly 36, 8-26.

Organisation for Economic Cooperation and Development (2004), Messages from PISA 2000. Paris: Author.

Organisation for Economic Cooperation and Development (2010a), PISA 2009 results: Learning to learn-Student engagement, strategies and practices (Volume III). Paris: Author. [Online: http://www.oecd.org/ dataoecd/11/17/48852630.pdf] (accessed May 2015).

Organisation for Economic Cooperation and Development (2010b), PISA 2009 results: Overcoming social background - Equity in learning opportunities and outcomes (Volume II). Paris: Author. [Online: http:// www.oecd.org/pisa/pisaproducts/48852584.pdf] (accessed May 2015).

Organisation for Economic Cooperation and Development (2012), Untapped skills: Realising the potential of immigrant students. Paris: Author.

Schofield, Janet W. \& Bangs, Ralph (2006), Conclusions and further perspectives. In: Schofield, Janet W., Migration background, minority-group membership and academic achievement: Research evidence from social, educational, and developmental psychology. AKI Research Review 5. Berlin: Programme on Intercultural Conflicts and Societal Integration 
(AKI), Social Science Research Center, 93-102. [Online: http://www. wzb.eu/zkd/aki/files/aki_research_review_5.pdf.] (accessed May 2015).

Stanat, Petra \& Christensen, Gayle (2006), Where immigrant students succeed: A comparative review of performance and engagement in PISA 2003. Paris: OECD.

Steele, Claude M. (1997), A threat in the air: How stereotypes shape intellectual identity and performance. American Psychologist 52, 613-629. [Online: http://dx.doi.org/10.1037/0003-066X.52.6.613] accessed May 2015).

Sullivan, Alice \& Brown, Matt (2013), Social inequalities in cognitive scores at age 16: The role of reading. London: Centre for Longitudinal Studies, Institute of Education, University of London. [Online: www.cls.ioe. ac.uk] (accessed May 2015).

UNESCO (2009a), Policy guidelines on inclusion in education. Paris: Author. [Online: http://www.scribd.com/doc/37626440/UNESCOEducation-Inclusion-Policy-Guidelines] (accessed May 2015).

UNESCO (2009b, December, 9-11), Tenth meeting of the Working Group on Education for All (EFA): Concept paper on marginalization. [Online: http://www.unesco.org/education/EFAWG2009/conceptpaper_marginalization.pdf $]$ (accessed May 2015). 


\section{Johanna Schnuch \\ Inklusion und Mehrsprachigkeit: Die Rolle von Sprachbewusstheit im multilingualen Spracherwerb}

The idea of inclusive education implies that schools need to adapt to the heterogeneous situations of their pupils, also with regard to the linguistically diverse backgrounds. There is an ongoing discussion about multilingual children and their abilities when it comes to language learning. A prevailing notion is that bilingual children may be especially successful in learning new languages due to their heightened metalinguistic skills, which should serve them in learning how to read and write and to prosper in school. However, it seems to depend on a variety of factors whether a multilingual child will be able to use his/her former linguistic knowledge constructively. This article discusses the role of language awareness. It will be explained why study results may seem to be inconsistent at first and how they could be plausibly interpreted. At last, some ideas will be presented as to how the development of language awareness could possibly be supported in the language-learning classroom in order to serve as a future learning device.

\section{Einleitung: Mehrsprachigkeit und Sprachenlernen}

Kindliche Mehrsprachigkeit wurde in deutschen Schulen lange Zeit eher als Problem denn als Normalität oder gar Bereicherung wahrgenommen. Die demographische Entwicklung und hohe Anzahl an mehrsprachigen Kindern erfordert jedoch ein Umdenken von Schule und Unterricht. Inklusion im Hinblick auf Mehrsprachigkeit bedeutet, dass sich ein Wandel vollziehen muss, vom traditionell monolingualen hin zu einem multilingualen Selbstverständnis unserer Schulen. Lehrmaterialien und Unterricht dürfen nicht ausschließlich für einsprachig deutsche Kinder konzipiert sein, denn diese Lernergruppe stellt längst nicht mehr die Norm dar. Vielmehr sollte sich beides an der sprachlichen Vielfalt in den Klassenzimmern orientieren (vgl. Atanasoska 2011: 178ff.). Dem Englischunterricht kommt dabei eine ganz besondere „Verantwortung für den Erfolg des nachfolgenden Sprachenlernens“ zu (Lenz 2009: 47). Er kann und sollte „Fenster öffnen“ und Kinder für andere Sprachen und Kulturen sensibilisieren (vgl. Nieweler 2001: 3). 
In Bezug auf Mehrsprachigkeit und Sprachenlernen und die Debatte um die Rolle und das Prestige von Migrationssprachen in unserer Gesellschaft wird in den letzten Jahren immer wieder die Frage diskutiert, ob es mehrsprachigen Kindern nun besonders leicht fällt, Englisch und weitere Sprachen zu lernen, oder ob sie damit vielleicht überfordert sind (vgl. Elsner in diesem Band). Ein positiver Aspekt, der häufig im Zusammenhang mit mehrsprachigen Kindern und Sprachenlernen genannt wird, ist ihre Sprachbewusstheit oder Language Awareness. Die guten Ergebnisse mehrsprachiger Kinder in Studien zum weiteren Sprachenlernen werden oft damit erklärt, dass sie „wohl [...] ein breiteres Repertoire an (meta-) sprachlichem Vorwissen besitzen als monolinguale“ (Hoti, Müller, Heinzmann, Wicki \& Werlen 2009: 22). Man geht also davon aus, dass mehrsprachige Kinder über eine erhöhte Sprachbewusstheit verfügen. Gleichzeitig wird der Sprachbewusstheit eine hohe Relevanz für das weitere Sprachenlernen zugeschrieben. Sie gilt zudem als besonders nützlich für das Lesenlernen (vgl. Dirim \& Müller 2007: 9; Jessner 2009; Rauch, Naumann \& Jude 2011; Riehl 2006: 19; Wolff 1997). Mehrsprachige Kinder müssten bzw. könnten also hervorragende Leser und Sprachenlerner sein. Trotzdem zeigen Studien, dass dies nicht grundsätzlich und für alle Kinder zu gelten scheint (vgl. Elsner in diesem Band). Ob es mehrsprachigen Kindern tatsächlich gelingt, ihre Mehrsprachigkeit für das weitere Sprachenlernen positiv nutzbar zu machen, scheint sehr unterschiedlich zu sein. In diesem Artikel soll untersucht werden, welche Rolle Sprachbewusstheit dabei einnimmt und wie die unterschiedlichen Ergebnisse in Bezug auf diesen Faktor möglicherweise zu erklären sind. Nachdem zunächst das Konstrukt sowie einige wichtige Studienergebnisse genauer dargestellt und diskutiert werden, ergibt sich zuletzt die Frage, welche didaktischen Schlussfolgerungen aus den Erkenntnissen gezogen werden können und was Sprachbewusstheit für die Inklusion von Mehrsprachigkeit in der Schule bedeutet bzw. wie sie konkret gefördert werden kann.

\section{Mehrsprachigkeit $=$ mehr Sprachbewusstheit?}

In der aktuellen Diskussion besteht ganz augenscheinlich eine „Tendenz, mehrsprachigen Kindern pauschal überdurchschnittliche metasprachliche Fähigkeiten zuzuschreiben“ (Krafft 2014: 155). Dies ist aus zwei Gründen zumindest mit einiger Vorsicht zu betrachten: 
Laut Krafft (2014) basiert diese Annahme oftmals auf „Einzelbeobachtungen“ oder der „Übertragung von Untersuchungsergebnissen“, die in Kontexten entstanden sind, die den hiesigen Bedingungen von Kindern mit Migrationshintergrund nicht entsprechen (Krafft 2014: 155). Man bedenke, wie divers die Sprachlernsituationen mehrsprachiger Kinder sind und dass es nicht den einen Mehrsprachigen gibt (vgl. Elsner in diesem Band). Zwar zeigen Erkenntnisse aus der Bilingualismusforschung durchaus, dass Kinder, die zu Hause mit zwei Sprachen aufwachsen, über besonders ausgeprägte metalinguistische Fähigkeiten verfügen (vgl. Cenoz 2003; Friesen \& Bialystok 2012; Hufeisen 2003; Jackson 2014: 221; Jessner 2009: 37) und auch für SchülerInnen, die am bilingualen Unterricht teilnehmen, scheint dies zu gelten (vgl. Fehling 2008). Es stellt sich jedoch die Frage, in wie weit diese Ergebnisse auf die spezielle Situation mehrsprachig aufwachsender Kinder im Migrationskontext an deutschen Schulen übertragbar sind (vgl. Portnaia 2007).

Zweitens sollte hinterfragt werden, was unter dem Konstrukt „metalinguistische Fähigkeiten“ eigentlich verstanden wird. Eine empirische Überprüfung der oben dargestellten Annahme wird durch „die fehlende Konturierung des Konzepts ,Sprachbewußtheit““ erschwert (Knapp-Potthoff 1997: 9) - und steht deshalb m.E. weiterhin aus. Der gesamten Diskussion über Unterschiede in der metasprachlichen Entwicklung mono- und bilingualer Kinder liegt letztlich die Annahme zugrunde, dass es sich bei dem Begriff metalinguistic ability um ein einheitliches Konstrukt handle (Bialystok 2001: 170). Seit den 70er Jahren wurden verschiedenste Konzeptualisierungsversuche unternommen und doch findet sich in der Literatur noch immer eine Vielzahl unterschiedlicher Termini, die von Sprachbewusstsein über metasprachlichel metalinguistische Bewusstheit, Sprachbegegnung oder Sprachsensibilisierung, Sprachwissen bis zu Sprachgefühl reicht. Häufig werden auch im deutschen Kontext die englischen Termini verwendet. Besonders umfassend sind dabei wohl die Definitionen für den englischen Begriff Language Awareness, der im deutschen meist mit Sprachbewusstheit übersetzt wird (vgl. z.B. Dechert 1997; Gnutzmann 1997; Hohm 2005; Kirschhock 2004: 62; Knapp-Potthoff 1997; Krafft 2014; Morkötter 2005; Oomen-Welke 2003: 2).

[D]er fachliche Terminus Sprachbewusstheit bedeutet, dass auf der Grundlage des aufmerksamen Gebrauchs einer oder mehrerer Sprachen Regularitäten, Beziehungen (sowie aktuell Unerklärbares) wahrgenommen, kognitiv bearbeitet 
und Einstellungen dazu entwickelt werden; er meint den bewussten Zugriff auf Sprachliches und die Beschäftigung mit de-kontextualisierten, aus dem Handlungszusammenhang herausgelösten sprachlichen Einheiten, die bewusst restrukturiert werden. (Oomen-Welke 2003: 2)

Mit dieser Definition unternimmt Oomen-Welke (2003) den Versuch, größtmögliche Klarheit über ein Konstrukt herzustellen, über das, wie bereits angemerkt, nicht unbedingt ein einheitliches Verständnis herrscht und das deshalb auch schwer operationalisierbar ist. Die Definitionen sind z.T. sehr weit gefasst. So gehen James \& Garrett (1995) von fünf verschiedenen Dimensionen von Language Awareness aus, die von der kognitiven über die affektiv-motivationale bis zur politischen Dimension reichen. ${ }^{1} \mathrm{Die} \mathrm{Au}-$ toren definieren Language Awareness als „a person's sensitivity to and conscious awareness of the nature of language and its role in human life" 2 (ebd.: 4) und beziehen dies auf ein ganzes Bildungsprogramm (ebd.: 306). Die affektive Dimension umfasst ihnen zufolge Motivationen und Einstellungen/Haltungen gegenüber einer Sprache. Die soziale Dimension „relates to social harmonisation in potentially divisive contexts of language variation and diversity“ (James \& Garrett 2002: 331), spiegelt also Gedanken und Gefühle gegenüber fremden Sprachen wider oder untersucht das gesellschaftliche Prestige von Sprachen (vgl. Morkötter 2005: 30). Mit der kognitiven Dimension ist die „awareness of pattern, contrast, system, units, categories, rules of language“ (James \& Garrett 2002: 331) gemeint. Sie umfasst auch die analytische Kompetenz, sich die Bedeutung unbekannter Wörter zu erschließen und verwendete Sprachlernstrategien zu benennen. „Es geht um das Erkennen von sprachlichen Regeln und Regelmäßigkeiten, von Kontrasten und von sprachlichen Einheiten auf den verschiedenen sprachlichen Ebenen“ (Morkötter 2005: 31; vgl. Rauch et al. 2011: 405). Die politische Dimension (auch als Critical awareness bezeichnet) behandelt den manipulativen Aspekt von Sprache, die z.B. für politische Zwecke missbraucht wird. Der kritische Umgang mit Texten, „,insbesondere im Hinblick auf den Zusammenhang von Sprache und Macht [steht] im Vordergrund“

1 Vgl. auch Breidbach, Elsner \& Young (2011).

2 Diese Definition entstand in der Language-Awareness-Arbeitsgruppe des $\mathrm{Na}$ tional Council for Language in Education (NCLE) (vgl. Donmall 1985: 7, zit. nach James \& Garrett 1995: 4). 
(Morkötter 2005: 30). Die Dimension der Performanz untersucht schlussendlich, ob „knowledge accruing from LA“ zu besserem Sprachenlernen führt: Geht erhöhte Language Awareness also mit höheren Sprachfähigkeiten einher? Wie spielen deklaratives und prozedurales Wissen zusammen? (vgl. James \& Garrett 2002: 331f.). Es geht den Autoren bei ihrem Konzept also nicht nur darum, Sprache und Inhalt auf der kognitiven Ebene getrennt betrachten zu können und über konkrete sprachliche Strukturen und Regularitäten zu reflektieren, sondern zum Beispiel auch darum, zu hinterfragen, welche Rolle bestimmten Sprachen in der Gesellschaft zukommt und wie Sprache zur Manipulation eingesetzt wird. Dieses sehr weite Verständnis von Language Awareness integriert zweifellos wichtige Bereiche, erschwert jedoch auf der anderen Seite eine klare Operationalisierung des Konstrukts.

Der deutsche Begriff Sprachbewusstheit (vgl. Oomen-Welke 2003) scheint v.a. auf eine der genannten Dimensionen, nämlich die kognitive Domäne zu rekurrieren, also das bewusste Erkennen von sprachlichen Regeln und Regelmäßigkeiten, Kontrasten und sprachlichen Einheiten (James \& Garrett 2002: 331; vgl. Morkötter 2005: 31; Rauch et al. 2011: 405).

Doch nicht nur in Bezug auf das Begriffsverständnis herrscht eine gewisse Uneinigkeit. Auch ist nicht abschließend geklärt, welche Rolle das explizite, bewusste Thematisieren sprachlicher Phänomene in diesem Konstrukt spielt. Bedeutet Sprachbewusstheit, dass tatsächlich über Sprache gesprochen wird? Welchen Einfluss hat das implizite Sprachwissen? Während für Vertreter der Bewegung aus Großbritannien das Explizit-machen ein definierendes Element von Language Awareness darstellt (James \& Garrett 1995: 306), verstehen andere Autoren unter dem Begriff bereits das implizite Wissen (vgl. Nicholas 1995: 79). ${ }^{3}$ Das Verständnis von Bewusstheit scheint sich auf einem Kontinuum zu bewegen, wobei die Grenzen zwischen explizit und implizit oftmals nicht klar gezogen werden (vgl. James \& Garrett 1995: 18). Eine Folge dieser Problematik ist, dass wenig Konsens über angemessene Erhebungsmethoden herrscht (Bialystok 2001: 170). Je

3 „[...]outside Britain, LA has even been seen in terms of the acquisition of implicit knowledge rather than explicit knowledge [...] the evidence for which is observed not in metalinguistic performance, but solely in linguistic performance“. (James \& Garrett 1995: 7) 
nachdem welches Verständnis einer Untersuchung zugrunde gelegt wird, werden ganz unterschiedliche Testverfahren verwendet.

Die fehlende Konturierung des Konzepts ,Sprachbewußtheit' macht es für empirische Untersuchungen schwer zugänglich und erlaubt im Grunde kaum eine Überprüfung der Annahme, die Förderung von Sprachbewußtheit sei nützlich für das Fremdsprachenlernen. (Knapp-Potthoff 1997: 9)

Es ist wichtig, dies im Hinterkopf zu behalten, wenn Erkenntnisse in Bezug auf die Sprachbewusstheit mehrsprachiger Kinder betrachtet werden. Im Folgenden sollen dennoch einige Studienergebnisse vorgestellt werden.

\section{Studienergebnisse zu Bilingualismus und Sprachbewusstheit}

Galambos \& Goldin-Meadow (1990) untersuchten die Entwicklung metalinguistischer Bewusstheit bei bilingual sowie monolingual aufwachsenden Kindern im Alter zwischen 4 und 8 Jahren anhand eines grammaticality judgment-Tests (d.h. anhand einer Aufgabe bei der die grammatikalische Richtigkeit von Sätzen zu beurteilen ist). Ihre Ergebnisse zeigen, dass die Fähigkeiten monolingual aufwachsender Kinder hier dieselbe Entwicklung durchlaufen wie die der bilingual aufwachsenden: Zunächst liegt der Fokus aller Kinder allein auf der Bedeutungsebene von Sprache und kann erst später auf die formale Ebene gerichtet werden. Die Erfahrung mit Mehrsprachigkeit scheint diese Entwicklung jedoch zu beschleunigen (Galambos \& Goldin-Meadow 1990: 49). Auch Friesen \& Bialystok (2012) betonen, dass bilinguale Kinder stärker darin sind, Form und Bedeutungsebene zu trennen und schon sehr früh ein Verständnis über die arbiträre Beziehung zwischen Sprache und Referent entwickeln (ebd.: 49). Hermanto, Moreno \& Bialystok (2012) zeigen auch bei Kindern, die zwar nicht in der Familie zweisprachig aufgewachsen sind, dafür aber bilingualen Unterricht besuchen, dass sich ihre metalinguistischen Fähigkeiten schneller entwickeln als die monolingualer Kinder (ebd.: 10; vgl. auch Fehling 2008). Tatsächlich weist die Mehrheit der Studien in diesem Bereich in Richtung eines Vorteils mehrsprachiger Kinder.

Doch es gibt durchaus auch Studien zur metasprachlichen Bewusstheit, bei denen mehrsprachige schlechter abschneiden als monolinguale Kinder (vgl. Bialystok 2001: 170). So zeigen die von Triarchi-Hermann (2006) untersuchten, im deutschsprachigen Migrationskontext aufwachsenden mehrsprachigen Kinder der dritten Klasse - in drei verschiedenen Tests 
zur metalinguistischen Bewusstheit (Phonologische Bewusstheit, Wortkonzept und Grammatik) - schlechtere Ergebnisse als ihre monolingualen MitschülerInnen. Auch bei Krafft (2014) schnitten die mehrsprachigen Kinder der vierten Jahrgangsstufe in vielen Bereichen der metalinguistischen Bewusstheit schlechter ab als die einsprachig aufgewachsenen Kinder. (Der Autor testet Grammatikalitätsurteile, den Wortbegriff und Segmentierungsfähigkeit.) Wie können diese unterschiedlichen Ergebnisse erklärt werden?

\section{Metalinguistische Fähigkeiten, Analyse und Kontrolle}

Ein Grund für die scheinbar widersprüchlichen Ergebnisse in Bezug auf Mehrsprachigkeit und Sprachbewusstheit ist, wie bereits angemerkt, auf die Problematik der Operationalisierung des zugrundeliegenden Begriffs und die daraus resultierende Varianz an Erhebungsmethoden zurückzuführen. Bialystok (2001) nimmt deshalb die folgende Unterscheidung vor, um den Begriff der metalinguistischen Fähigkeiten genauer zu differenzieren. Die Autorin geht davon aus, dass dem Bearbeiten metasprachlicher Aufgaben jeweils zwei verschiedene kognitive Prozesse zugrunde liegen. Beide, d.h. sowohl Analyse- als auch Kontrollprozesse, sind demnach für die Bearbeitung metalinguistischer Aufgaben wichtig, spielen aber je nach Art der Aufgabe eine unterschiedlich starke Rolle. Bialystok (2001) zufolge haben bilinguale Kinder in gerade solchen Aufgaben einen Vorteil, die hohe kognitive Kontrollfähigkeiten erfordern. Selbst die sogenannten „elitär mehrsprachigen “4 Kinder haben ihrzufolge also keine „across the board metalinguistic abilities“ und schneiden deshalb auch nicht grundsätzlich besser in metalinguistischen Tests ab (vgl. Barac \& Bialystok 2012: 413; Friesen \& Bialystok 2012: 54; Jessner 2008: 30).

Unser (sprachliches) Wissen ist als mentale Repräsentation gespeichert und kann in mehr oder weniger analysierter Form vorliegen. Der Prozess der Analyse bezeichnet die Fähigkeit, implizites Wissen zu strukturieren, abstraktere, symbolische mentale Repräsentationen zu konstruieren. Die mentalen Repräsentationen werden dadurch expliziter und der Bewusstsein/

4 D.h. Mehrsprachige, die von Geburt oder frühem Kindesalter an durch bilinguale Erziehung aufwachsen (vgl. Wolff 2010). 
Introspektion zugänglicher (Bialystok 2001: 177). Die exekutive Kontrolle steuert und richtet die Aufmerksamkeit auf ganz bestimmte Aspekte eines sprachlichen Stimulus. Sie wird vor allem bei widersprüchlichen Signalen benötigt, d.h. wenn die Aufmerksamkeit selektiv auf eine bestimmte Information gerichtet werden muss, während eine ablenkende Information ausgeblendet wird (Bialystok 2001: 178). Man geht davon aus, dass die zentrale Exekutive z.B. für die Sprachwahl zuständig ist und infolgedessen bei Mehrsprachigen besonders gut ,trainiert' ist - denn stets muss eine der beiden aktiven Sprachen quasi unterdrückt werden, da sie je nach Situation gerade nicht gebraucht wird (Friesen \& Bialystok 2012: 49).

The bilingual advantage $[\ldots]$ is in the ability to control attention when there is misleading information. [...] The effect of bilingualism on children's development is that it enhances their ability to attend to relevant information in the presence of misleading distractions. (Bialystok 2001: 179f.)

Mehrsprachige Kinder besitzen demzufolge besonders starke exekutive Kontrollfähigkeiten (vgl. Galambos \& Goldin-Meadow 1990: 49f. ${ }^{5}$ ). Diese nützen ihnen auch bei einigen metalinguistischen Testaufgaben - aber eben nicht bei allen gleichermaßen. Nach Bialystok (2001) erfordern all jene Tests eine erhöhte kognitive Kontrolle, bei denen nicht nur die formale Ebene von Sprache fokussiert, sondern parallel die Bedeutungsebene ignoriert werden muss. Dazu gehören z.B. das Zählen von Wörtern in sinnhaften Sätzen, Grammaticality judgment von sinnfreien Sätzen, aber auch die Lautanalyse von Wörtern (Phonemsegmentation) (ebd.: 179).

Neben der exekutiven Kontrolle spielen jedoch noch weitere Faktoren bei der Bearbeitung metalinguistischer Aufgaben eine Rolle. Für viele Testaufgaben der sprachlichen Bewusstheit ist ein gewisser sprachlicher Level erforderlich. Hierzu gehören z.B. die häufig eingesetzten grammaticality judgment-Tests, bei denen die grammatikalische Richtigkeit einer Äußerung

5 Wie auch Bialystok (2001) gehen die Autoren davon aus, dass der Prozess, der den Kindern hilft, ihre Sprachen automatisch zu differenzieren auch für ihre höhere sprachliche Bewusstheit, ihre "heightened attention to the form of language (as opposed to its meaning)" verantwortlich ist. Wenn es jedoch darum geht, sprachliche Verstöße zu beschreiben, ist dieser automatisierte Prozess wenig hilfreich, weshalb monolinguale und bilinguale Kinder bei der Aufgabe, Fehler zu erklären, ähnlich gut abschneiden (Galambos \& Goldin Meadow 1990: 49f.). 
bewertet werden muss (vgl. Davidson \& Raschke 2009: 5). Jude (2008) macht darauf aufmerksam, dass „[s]pezifische Teilkompetenzen, wie eine ausgefeilte Sprachbewusstheit [...], erst [dann] beobachtet werden [können], wenn ein grundlegender Wortschatz vorhanden ist“ (ebd.: 22). Dabei ist zu beachten, dass der Wortschatz von bilingualen Kindern in den jeweiligen Einzelsprachen normalerweise kleiner ist als der monolingualer Kinder (Bialystok 2011). Erfordert eine Aufgabe in metalinguistischen Tests also eine hohe Sprachkompetenz (Wortschatz oder Grammatikwissen), so haben mehrsprachige Kinder möglicherweise sogar einen Nachteil gegenüber monolingualen (Friesen \& Bialystok 2012: 52; vgl. Davidson \& Raschke 2009; vgl. Krafft 2014) - je nachdem in welcher Sprache der Test durchgeführt wird. „[T]asks, that favor the recruitment of formal language knowledge may not result in a bilingual advantage“" (Friesen \& Bialystok 2012: 49).

Bei Aufgaben, die vor allem hohe Anforderungen an die zentrale Exekutive stellen, ist es weniger relevant, in welcher der beherrschten Sprachen dies geschieht, da die zentrale Exekutive zum kognitiven System gehört und deshalb sprachenunabhängig agiert. Friesen \& Bialystok (2012) zeigen, dass bilinguale Sprecher den Nachteil, der ihnen unter Umständen durch hohe sprachliche Anforderungen einer Aufgabe entsteht, sogar ausgleichen können, wenn gleichzeitig auch besonders starke exekutive Kontrollfähigkeiten gefordert werden: „When a task enables bilinguals to make use of their superior executive control skills, these skills will offset the costs of weaker language knowledge" (ebd.: 50). Dies allerdings konnte in einer Studie für den deutschen Migrationskontext nur für jüngere Kinder (der ersten Jahrgangsstufe) bestätigt werden. In der Erhebung von Krafft (2014), in welcher mit deutschsprachigen Instrumenten gearbeitet wurde, zeigen mehrsprachige Kinder im Vergleich zu monolingual aufwachsenden insgesamt Defizite in den metalinguistischen Bereichen, die vom sprachlichen Wissen in der Zweitsprache Deutsch abhängen. Die potentiellen Vorteile mehrsprachiger Kinder zeigen sich hier nur zu Schulbeginn. Am Ende der Grundschulzeit sind auch in den Bereichen, in denen es v.a. um Kontrollfähigkeiten geht, keine Vorteile mehr zu erkennen ${ }^{6}$ (Krafft 2014: 156).

6 Dies betrifft Grammatikalitätsurteile semantisch unsinniger Sätze und lexikalische Segmentierung. 


\section{Sprachbewusstheit mehrsprachiger Kinder im Migrationskontext}

Sprachbewusstheit entwickelt sich erfahrungsbasiert, im Umgang mit Sprachen. Mehrsprachige Kontexte bergen deshalb eigentlich ein besonders großes Förderpotential. Da sie natürlicherweise viele Gelegenheiten zum Sprachvergleich bieten, sollte sich Sprachbewusstheit hier besonders gut entwickeln können (Oomen-Welke 2003: 2). Trotzdem scheint dies für den deutschen Schulkontext - jedenfalls im Hinblick auf die von Krafft (2014) angewendeten Testverfahren - nur eingeschränkt zu gelten. Dies ist einerseits, wie bereits erwähnt, mit der uneinheitlichen zugrundeliegenden Definition des Konstrukts „Sprachbewusstheit“ und daraus resultierend den unterschiedlichen Erhebungsmethoden zu erklären. Eine wichtige Rolle spielt aber auch der spezifische Erhebungs- bzw. Spracherwerbskontext, z.B. die migrationsbedingte Mehrsprachigkeit (vgl. Elsner in diesem Band). Es hat sich gezeigt, dass Mehrsprachigkeit nur „unter bestimmten Bedingungen zu einer höheren Sprachbewusstheit und damit zu einer höheren Sprachlernfähigkeit“ führt (Wolff 2010: 188). Während die Bewusstheit von Kindern, die „elitär mehrsprachig“ aufwachsen, durch die symmetrische Förderung beider Sprachen und ihrer bilingualen Literacy bereits in der Familie gestärkt wird, erhalten Kinder, die im Migrationskontext aufwachsen, oft keine ausreichende Förderung in beiden Sprachen - geschweige denn die Förderung ihrer Möglichkeiten, über Sprache und sprachliche Strukturen zu reflektieren. Lesen und Schreiben lernen sie meist zunächst nur in einer Sprache, die sie möglicherweise nicht einmal altersgemäß beherrschen, nämlich der Majoritätssprache Deutsch. Dies hat negative Konsequenzen - auch für die allgemeine Sprachbewusstheit der Kinder:

Das Ergebnis für diese Form von Mehrsprachigkeit ist eine sehr viel weniger ausgebildete Sprachbewusstheit, die unter der von monolingualen Sprechern liegt und zu großen Problemen beim Erwerb weiterer Sprachen führt. (Wolff 2010: 188)

Man geht gemeinhin davon aus, dass Menschen, die einmal eine andere Sprache gelernt haben, besonders gut darin sind, „Sprachverarbeitungsund Sprachlernstrategien bewusst zu benennen, zu beschreiben und bei ihren Sprachlernprozessen einzusetzen“ (ebd.: 187). Dies scheint erst einmal für alle Formen von Mehrsprachigkeit zu gelten (vgl.: ebd.), d.h. auch für Kinder, die im Migrationskontext mehrsprachig aufwachsen. Jedoch 
muss ihnen die Gelegenheit dazu geboten werden. Sie müssen dazu angeregt werden, über Sprache zu reflektieren, benötigen spezifische Impulse (vgl. Oomen-Welke 2011).

Was das Sprachenlernen angeht sind mehrsprachige Kinder sicherlich kein unbeschriebenes Blatt. Auch wenn sie ihre bisherigen Sprachen nicht unter institutionellen, strukturierten Bedingungen ${ }^{7}$ sondern eher unsystematisch und v.a. im familiären Kontext erworben haben (vgl. Portnaia 2007: 108), so haben sie ganz bestimmte kognitive und emotionale Vorerfahrungen mit dem Sprachenlernen gemacht. Wahrscheinlich kennen sie das Gefühl, nicht alles auf Anhieb verstehen zu müssen, sind vielleicht toleranter oder selbstsicherer, wenn sie einmal nicht wissen, was ein Wort konkret bedeutet. Auch haben sie möglicherweise schon Erfahrungen damit, wie man neue Worte am besten lernt, wissen vielleicht eher um den eigenen Lerntyp oder haben bestimmte Strategien entwickelt - zum Beispiel, dass es helfen kann, auf andere Sprachen zurückzugreifen (Hoti et al. 2009: 6; Hufeisen \& Gibson 2003: 17). Dennoch sollte man sowohl auf der kognitiven, sprachstrukturellen als auch auf der affektiv-motivationalen Ebene sehr vorsichtig mit derlei Annahmen sein. Kann man wirklich grundsätzlich davon ausgehen, dass Kinder, die mit mehreren Sprachen aufwachsen, prinzipiell motivierter oder weniger schnell überfordert sind und beim Lernen weiterer Sprachen einen Vorteil haben? (vgl. Hoti et al. 2009: 22f.; vgl. Riehl 2006: 19). Wenn man bedenkt, dass der von Gogolin (1994) postulierte monolinguale Habitus in deutschen Bildungseinrichtungen doch noch weit verbreitet ist (vgl. Kollmeyer 2007; Oomen-Welke \& Pena Schumacher 2001; vgl. Elsner in diesem Band), muss man davon ausgehen, dass nicht alle Kinder ihre Mehrsprachigkeit wertschätzen gelernt haben und dem Sprachenlernen vielleicht nicht nur positiv gegenüber eingestellt sind. Nicht wenige SchülerInnen haben in Kindergarten und Schule schwierige und demotivierende Sprachlernerfahrungen machen müssen. Daraus resultiert, dass Sprachbewusstheit, die „in Migrationskontexten oder in bestimmten Formen des Fremdsprachenunterrichts entstanden [ist,] verschüttet“ (Wolff 2010: 189). Vielen mehrsprachigen Kindern fällt es schwer, dieses verborgene Potential eigenständig

7 Die Tertiärsprachenforschung beschäftigt sich v.a. mit den Sprachlernstrategien und Transferprozessen erfahrener Lerner, mit typologisch verwandten Sprachen und dem systematischen Lernen von weiteren Fremdsprachen. 
zu nutzen. Es ist also wichtig, sie bei dem Prozess der Bewusstwerdung zu unterstützen und mehrsprachige Kinder im Unterricht so zu fördern, dass sie ihre Fähigkeiten für das weitere Sprachenlernen einsetzen können (vgl. ebd.).

\section{Welche Rolle spielt die Sprachbewusstheit für weiteres Sprachenlernen?}

Lenz (2009) beschreibt den Zusammenhang von Sprachbewusstheit und Mehrsprachigkeit als einen „Kreislauf“:

Beides kann gleichermaßen als Voraussetzung und auch als Ziel des jeweils anderen Konzeptes verstanden werden. Um Mehrsprachigkeit zu erzielen, erscheint die Förderung von Sprachbewusstheit essentiell [...] Sprachbewussten Lernern fällt es in der Folge leichter, weitere Sprachen zu lernen [...] Wer mehrsprachig ist, hat wiederum erhöhte Chancen, sprachbewusster zu werden, da Lernende einen Einblick in verschiedene Sprachsysteme erhalten, womit sich der Kreis letztendlich schließt. (ebd.: 46)

Man geht also einerseits davon aus, dass mehrsprachige Lerner eine erhöhte Sprachbewusstheit besitzen (können), andererseits scheint Sprachbewusstheit förderlich für weiteres Sprachenlernen zu sein: „(Meta-) linguistic consciousness-raising activities“, d.h. ein dekontextualisierter, aus dem Handlungszusammenhang gelöster Umgang mit Sprache und ein Fokus auf die Formebene der Sprache stehen mit "efficiency (and accuracy) in second/ foreign language learning" in Verbindung (Roberts 2011: 258). Dieser Bewusstmachungsprozess, das Richten der Aufmerksamkeit auf die formale Ebene (attention to form), steht dabei nicht im Widerspruch zu Prinzipien des modernen kommunikativen Fremdsprachenunterrichts. Es geht bei der Förderung von Sprachbewusstheit nicht darum, dass Lerner zu Beginn des Lernprozesses erst einmal fokalisiert die grammatischen Regeln und Strukturen einer Sprache lernen, wie es vielleicht im traditionellen fremdsprachlichen Grammatikunterricht das Ziel war. Vielmehr entwickeln sie zunächst eine periphere Form der Sprachbewusstheit. ${ }^{8}$ Diese wird dann im Verlauf des Spracherwerbsprozesses weiter entwickelt und sukzessive

8 Mit der peripheren Bewusstheit nehmen wir Dinge um uns herum wahr, ohne den Fokus darauf zu richten. So achten wir z.B. beim Fahrradfahren nicht mehr darauf, was genau wir wann und warum tun. Trotzdem sind wir uns dessen zu einem gewissen Grad - eben peripher - bewusst (vgl. van Lier 1995: 3). 
fokalisiert. Das bedeutet, dass Lerner je nach Entwicklungsstand nach und nach ihre Aufmerksamkeit auf formale Phänomene der Sprache richten und „bestimmte sprachliche Bereiche auch in die fokale Bewusstheit heben“ (Wolff 2010: 189). Dies geschieht je nach Entwicklungsstufe innerhalb ihrer zone of proximal development (vgl. Robinson 2008: 264): Wenn kleinere Kinder oder Lerner zu Beginn des Lernprozesses Sprache betrachten, nehmen sie zunächst vor allem die Bedeutung und die Lautform von Wörtern wahr (Oomen-Welke 2011). Um die Aufmerksamkeit der Lernenden auf die Formebene zu richten, helfen sogenannte form-focused tasks, außerdem sollte der Input entsprechend gestaltet sein (Robinson 2008: 259). Wenn die inhaltliche Bedeutung der Wörter in einer Aufgabe entsprechend vorentlastet wird, sodass weniger Arbeitsspeicherkapazität (vgl. ebd.; Bialystok 2001) für die Verarbeitung des Inhalts aufgewendet werden muss, wird es den Lernenden erleichtert, ihren Fokus auf die Formebene zu richten (Roberts 2011: 258). Der Language Awareness-Ansatz bedeutet also keineswegs eine Rückkehr zur Grammatik-Übersetzungs-Methode, sondern setzt „an der Schnittstelle von implizitem und explizitem Lernen an“ (Lenz 2009: 49). Bewusstes Lernen sollte „als entdeckende und forschende Eigentätigkeit verstanden werden “ (Morkötter 2005: 31). Man geht heute davon aus, dass ein „gewisser focus on form in Kombination mit Sprachreflexion“ (Lenz 2009: 49) für die erfolgreiche Sprachentwicklung notwendig ist, weil sich gezeigt hat, dass neben den impliziten (d.h. unbewussten, beiläufigen) Lernprozessen eben auch explizitere (d.h. regelgeleitete) Lehr- und Lernformen hilfreich sein können. Die Aufmerksamkeit auf die Formebene sprachlicher Strukturen zu richten kann demnach das implizite Lernen unterstützen und für die prozedurale Kompetenz förderlich sein (vgl. ebd.).

Eine Förderung von Sprachbewusstheit hat jedoch nicht allein die Verbesserung der sprachlichen Kompetenz oder Performanz in einer Zweit-I Fremdsprache zum Ziel. Insbesondere mit Blick auf die verschiedenen Ebenen von James \& Garrett (1995) wird deutlich, dass die Förderung von Language Awareness auch „ein kognitives und affektives Ziel an sich“ (Morkötter 2005: 32) darstellt und sich positiv auf die Lernmotivation, die interkulturelle Kompetenz sowie die gesamte Persönlichkeitsentwicklung mehrsprachiger Lerner auswirkt (vgl. Muñoz 2014: 123). Außerdem wird die Sprachlernbewusstheit oder Language Learning Awareness gestärkt. Diese „umfasst den Aspekt der Bewusstheit des Erlernens von Sprachen 
und beinhaltet damit auch Kenntnisse und Fähigkeiten, die in Verbindung mit Sprachlernstrategien stehen “ (ebd.: 122), die wiederum hilfreich beim weiteren (Sprachen-) Lernen sind.

\section{Wie kann Sprachbewusstheit im Unterricht gefördert werden?}

Sprachbewusstheit lässt sich vor allem dadurch fördern, dass andere Sprachen in die allgemeine Reflexion über Sprache miteinbezogen werden [...] Dies reicht von der Schaffung und Aufrechterhaltung von Motivation und Interesse bis hin zur Vermittlung von (Sprach-)Lernstrategien. (Lenz 2009: 46f.)

Von der kognitiven bis zur affektiv-motivationalen Dimension von Language Awareness hat die Einbeziehung von weiteren Sprachen in den (Fremdsprachen-)Unterricht eine ganz besondere Bedeutung. Trotzdem scheinen sowohl die Herkunftssprachen der Lernenden als auch ihre zuvor gelernten Fremdsprachen bisher noch selten systematisch einbezogen zu werden. Gerade die Erstsprachen werden zum Teil „als Belastung bzw. als Problem und nicht als Ressource für den Fremdsprachenunterricht angesehen “ (Muñoz 2014: 121). Lernende haben nicht den Eindruck, ihre Mehrsprachigkeit würde von den Lehrkräften wertgeschätzt (ebd.: 123). Dies hat jedoch Folgen für den Lernprozess: Wenn ignoriert wird, dass Kinder bereits mit anderen Sprachen in Kontakt gekommen sind und Lehrkräfte ihren Unterricht an vermeintlich einsprachigen Lernern ausrichten, d.h. Bezug nur zur Mehrheitssprache hergestellt und nur auf deutsche Konzepte verwiesen wird, so verschwenden sie wertvolle Ressourcen (vgl. Jessner 2009: 130). Es kann gar „zu einer Blockierung der metasprachlichen Entwicklung zweisprachiger Kinder kommen“ (Muñoz 2014: 123). Gleichzeitig scheinen aber viele Lehrkräfte, die weitere Sprachen gern in ihren Unterricht einbeziehen würden, unsicher, wie dies zu realisieren sein kann. Denn bisher existieren kaum „explizite Ansätze zum Einbezug der lebensweltlichen Mehrsprachigkeit“ (Muñoz 2014: 121, 126) und so manifestiert sich speziell im Fremdsprachenunterricht auch weiterhin der monolinguale Habitus (Muñoz 2014: 121, vgl. Gogolin 1994).

Wie also kann ein Kontext geschaffen werden, in dem Mehrsprachigkeit wertgeschätzt wird? Wie können wir SchülerInnen darin unterstützen, ihre verborgenen Sprachlernerfahrungen langfristig gewinnbringend einzusetzen und eine Bewusstheit zu entwickeln, die ihnen auch beim weiteren Sprachenlernen von Nutzen sein kann? 
Eine Förderung von Language Awareness kann auf den ganz unterschiedlichen Ebenen (vgl. James \& Garrett 1995) geschehen. Auf der affektivemotionalen Ebene bzw. der Ebene der Sprachlernbewusstheit wird gemeinsam über die eigene Mehrsprachigkeit reflektiert. Den SchülerInnen wird bewusst, was sie in welcher Sprache bereits können, wie sie ihre Sprachen gelernt haben, was sie über die Sprachen wissen und wie sie ihre vorhandenen Sprachlernerfahrungen und -strategien für das weitere Sprachenlernen einsetzen können. Es ist wichtig, dass sie von den Lehrkräften darin unterstützt werden (vgl. Elsner \& Lohe 2015). Dies kann z.B. in Sprachenportfolios (vgl. z.B. Oomen-Welke \& Pena Schumacher 2001), Sprachentagebüchern oder Language Identity Texts (vgl. Cummins, o.J.) geschehen. Eine affirmative Einstellung zur eigenen Mehrsprachigkeit unterstützt die erfolgreiche Nutzung vorheriger Sprachkenntnisse (vgl. Hufeisen \& Gibson 2003: 20) und stärkt das sprachliche Selbstkonzept der Kinder. Es ist außerdem wichtig, den Kindern positive Sprachlernerfahrungen in ihren Zweitsprachen zu ermöglichen und gleichzeitig Mehrsprachigkeit im Unterricht wirklich als etwas Wertvolles anzuerkennen. Es ist motivierend, wenn Lernende erfahren, dass sie ihr bisheriges Sprachwissen und ihre Erfahrungen mit Mehrsprachigkeit im Unterricht nutzen und einbringen können. Und weil Sprache Teil ihrer Identität ist, wirkt sich dies positiv auf die gesamte Persönlichkeitsentwicklung aus (Muñoz 2014: 123).

Auch für Entwicklung der fokalen Sprachbewusstheit auf der kognitiven Ebene (d.h. dem Reflektieren über sprachliche Strukturen) ist es wichtig, mehrsprachige Kinder im Reflexionsprozess zu unterstützen. Der kommunikative task-based-Ansatz scheint besonders dazu geeignet, focus on form und Bedeutungsverhandlungen zu kombinieren und Kinder darin zu unterstützen, ihr vorhandenes metalinguistisches Wissen im Entschlüsselungsprozess einzusetzen (vgl. Roberts 2011: 265f.). Die Aufmerksamkeit der Lernenden wird während der Bearbeitung kommunikativer Aufgaben auf formale Aspekte von Sprache gelenkt. Auf diese Weise kann das Ziel erreicht werden,

die besonderen Stärken der mehrsprachigen Kinder hinsichtlich der distanzierten / deautomatisierten Sprachbetrachtung und der Kontrolle der sprachlichen Verarbeitung (control) zu nutzen und weiter anzuregen. (Krafft 2014: 156)

Wolff (2010) betont, wie wichtig für den Prozess der Entwicklung einer fokalen Bewusstheit die Förderung von Lese- und Schreibfähigkeiten in der Erstsprache der Kinder ist, um die sogenannten „akademischen 
Sprachfähigkeiten“9 auch in dieser Sprache weiterzuentwickeln (ebd.: 188). Auch die Untersuchung von Krafft (2014) zeigt deutlich, wie sehr die Sprachbewusstheit (allgemein bzw. in der L2) der Kinder tatsächlich von einer Förderung ihrer L1-Literacy zu profitieren scheint. Sein Ergebnis unterstützt die Annahme, dass Schreib- und Lesefähigkeiten in der Erstsprache eine Voraussetzung dafür sind, dass Sprachbewusstheit genutzt werden kann (vgl. Wolff 2010: 190). Robinson (2008) betont, wie wichtig die Entwicklung der metasprachlichen Fähigkeiten in der Erstsprache für weiteres Sprachenlernen ist:

It is only by using his metalinguistic skills developed through L1 to attend to and identify form-meaning relationships in the input - and to test out those relationships in a range of suitably graded activities - that the foreign language learner's interlanguage can progress. (Robinson 2008: 279)

Auch aus diesem Grund scheinen zum Beispiel sprachkontrastierende Vorgehen, die Sprachen einander gegenüberstellen, hilfreich zu sein: „[V]or allem durch kontrastives und komparatives, d.h. vergleichendes Sprachenlernern“ wird Lenz (2009: 49) zufolge die Verarbeitungstiefe erreicht, die erforderlich ist, damit deklaratives und prozedurales Wissen sich gegenseitig unterstützen. Durch kontrastivkomparierendes Arbeiten, d.h. beim Vergleichen der Parallelen und Unterschiede zwischen Sprachen wird „Sprachbewusstheit fokalisiert und zu einem Instrument für den Erwerb der neuen Sprache“ (Wolff 2010: 189). Die SchülerInnen lernen, wie sie ihr Wissen aus zuvor gelernten Sprachen als Strategie zum Erschließen und Erinnern von Bedeutungen (vgl. Lenz 2009: 51) selbstständig einsetzen können. „Auf diese Weise steigen die Chancen auf eine erhöhte Sprachbewusstheit und auf ein erfolgreiches Vokabellernen“ (Lenz 2009: 51).

Dabei geht es weniger darum, das Prinzip der Einsprachigkeit grundsätzlich zu hinterfragen, als vielmehr darum, die gezielte Einbeziehung anderer Sprachen als hilfreich anzuerkennen. Im mentalen Lexikon werden die Sprachen der Lerner ohnehin miteinander verknüpft (Nieweler 2001: 6): interlingualer Transfer ist also kaum zu verhindern, die Sprachen beeinflussen sich ganz automatisch gegenseitig. Man geht davon aus, dass „neue Wissenskomplexe mit bereits vorhandenen zusammengeführt werden“

9 Vgl. Cummins' (2008) Unterscheidung von BICS und CALP. 
(ebd.: 7). Deshalb ist es sinnvoll, verschiedene Sprachen auch in der Schule nicht völlig isoliert voneinander zu behandeln, sondern das sprachliche Vorwissen der Lernenden zu aktivieren und die Transferpotentiale insbesondere von Lexik und Grammatik systematisch in den Unterricht einzubeziehen. Auf diese Weise werden die SchülerInnen optimal bei der Konstruktion ihrer individuellen Lernersprachen unterstützt (ebd.).

Schon Kinder in der Grundschule können eigenständig Sprachen vergleichen, untersuchen und Unterschiede entdecken (vgl. Oomen-Welke 2011). Es gilt, sie für Gemeinsamkeiten und Differenzen in der Struktur verschiedener Sprachen zu sensibilisieren, ihre Neugier und Lust zu wecken mit Sprache zu spielen, gezielte Impulse zu setzen und ihnen damit auch beim weiteren Sprachenlernen zu helfen. Sicherlich ist eine gewisse Kenntnis auf Seiten der Lehrkraft über die Erstsprachen der Kinder dabei hilfreich. Aber es ist weder möglich noch notwendig, alle Sprachen der Kinder bis zur Perfektion zu erlernen. Viel wichtiger als das Beherrschen vieler Sprachen ist eine offene und positive Einstellung der Lehrperson gegenüber sprachlicher Vielfalt! Um Sprachvergleiche an geeigneter Stelle, sinnvoll und phasenweise in den Unterricht zu integrieren reicht es aus, ein paar Gemeinsamkeiten oder Unterschiede zu kennen bzw. kennen lernen zu wollen (Dirim \& Müller 2007: 12). Lehrkräfte aus dem muttersprachlichen Unterricht, die SchülerInnen selbst oder ihre Eltern können eine wichtige Hilfe und Kooperationspartner sein.

\section{Kinder als Sprachendetektive: konkrete Umsetzungsideen}

Die Möglichkeiten, Kinder zur Reflexion über Sprache(n) anzuregen, sind zahlreich. ${ }^{10}$ Wichtig ist, dass die Einbeziehung anderer Sprachen bewusst und strukturiert geschieht und durch spezifische Nachfragen des Lehrers begleitet wird. Wenn die vorhandene Sprachenvielfalt in der Klasse z.B. in morgendlichen Begrüßungsritualen, beim Singen von Geburtstagsliedern in mehreren Sprachen, in mehrsprachigen Geschichten/Texten etc. (vgl. Elsner \& Lohe 2015) einbezogen wird, so sollte die Bewusstheit der Kinder durch gezielte ,Detektivaufträge’ gefördert werden. Die Lehrperson unterstützt

10 Weitere Ideen zur Einbeziehung von Mehrsprachigkeit in den Unterricht finden sich bei Schnuch (2014), bei Schader (2012); vgl. auch Elsner (dieser Band); Elsner \& Lohe (2015). Es sei auch auf das JALING-Projekt (Fidler et al.: o.J.) verwiesen. 
die Bewusstmachung demnach durch konkrete Fragen: Was fällt euch auf? Welche Worte sind ähnlich? Was ist anders? So wird die Bewusstheit der Kinder auf ganz bestimmte Eigenschaften der Sprache(n) gelenkt und ein Reflexionsprozess angeregt.

Eine Förderung ist auf allen linguistischen Ebenen (Phonologie bis Pragmatik) möglich. So können die Aussprache und ,fremde' Laute genauso thematisiert werden wie grammatische Strukturen oder unterschiedliche Höflichkeitsformen: Im Englischen gibt es nur you, im Deutschen nutzen wir Sie oder $\mathrm{D} u$ - wie ist das in anderen Sprachen? Ein Verfahren, bei dem es v.a. um sprachübergreifenden Transfer rezeptiver Kompetenzen geht, ist der Ansatz der Interkomprehension, der sich bisher vor allem in den romanischen Sprachen durchgesetzt hat (Muñoz 2014: 125). Die Idee ist, dass Sprecher Texte in Sprachen, die mit ihrer L1 etymologisch verwandt sind, weitgehend verstehen können, ohne diese Sprache tatsächlich gelernt zu haben (z.B. jemand mit der Erstsprache Französisch versteht viele Wörter des Spanischen) (vgl. ebd.; Doyé 2004; Hufeisen \& Marx 2007). Im interkomprehensiven Unterricht werden v.a. Sprachen einer Sprachenfamilie einander vergleichend gegenübergestellt, sodass Transferbasen, d.h. Möglichkeiten des Transfers deutlich werden: „Neues kann identifiziert und ,in das mentale plurilinguale Lexikon’ integriert werden “ (Muñoz 2014: 125). Dabei geht es jedoch vor allem um die Vermittlung der entsprechenden Strategien und weniger um den Transfer an sich. Die SchülerInnen lernen Strategien kontrastiver Verfahren kennen, die sie eigenständig anwenden und auf anderes Sprachenlernen übertragen können (ebd.).

Weil angenommen wird, dass sogenannte Cognates auch im mentalen Lexikon gemeinsam repräsentiert sind, scheinen Sprachen mit lexikalischen Bereichen, die phonologische, orthographische und semantische Ähnlichkeiten aufweisen, für diese Art der sprachanalytischen Behandlung „besonders geeignet“ (Lenz 2009: 50). Durch das Erschließen und Memorieren orthographisch ähnlicher Begriffe wird der Wortschatz erweitert und eine Verbindung zwischen den Sprachen geknüpft. Werden diese Wörter häufig gemeinsam abgerufen, so vertiefen sich die Verknüpfungen, und in je mehr Sprachen Cognates vertreten sind, desto schneller können diese verarbeitet werden (ebd.: 52). Schon kleine Kinder reflektieren von sich aus die unterschiedliche Bedeutung ähnlich klingender Wörter und die Nähe der Sprachen, die sie kennen. Sie werden aber auch auf Unterschiede 
aufmerksam (vgl. Oomen-Welke 2011). Mehrsprachige semantische Felder entwickeln sich von Beginn des Lernprozesses an - auch mit Begriffen, die weniger Ähnlichkeiten aufweisen als Cognates.

Im Unterricht bietet es sich zum Beispiel an, über die Namen der Kinder (ihre Bedeutungen, ihre Herkunft) sowie über Namen von Figuren in Geschichten zu reflektieren. Warum bleiben diese in einigen Übersetzungen gleich und werden in anderen Geschichten geändert? Warum heißt Pippi eigentlich „Langstrumpf“ und wie heißt sie in anderen Sprachen? Wie lauten die Namen der verschiedenen Charaktere aus Harry Potter in der türkischen, russischen, englischen oder französischen Übersetzung? Und haben ihre Namen eine Bedeutung? Ähnlich kann mit Bezeichnungen von Gerichten und Zutaten für eine internationale Speisekarte verfahren werden. Auch auf Lebensmittelpackungen finden sich Beschriftungen und damit Wörter in verschiedenen Sprachen. Die morphologische Bewusstheit wird gefördert, wenn den Kindern auffällt, dass Orangensaft im Deutschen nur ein Wort ist, während es im Englischen (orange juice), Türkischen (portakal suyu) und im Polnischen (sok pomarańczowy) jeweils zwei Wörter und auf Französisch eigentlich drei (jus d'orange) sind. Woran liegt das? In welchen Sprachen kommt tatsächlich genau das Wort „Orange“ vor und wo kommt es her? Mithilfe eines Online-Wörterbuchs können die entsprechenden Übersetzungen in verschiedenen Sprachen gefunden werden, und im Anschluss auch die von Apfelsaft oder Tomatensaft. Dabei lässt sich vergleichen, welcher Teil des Begriffes in jeder Sprache jeweils gleich bleibt, und somit kann vielleicht sogar herausgefunden werden, welches Morphem Saft bedeutet. Auf ähnliche Weise kann aber auch erschlossen werden, wie die Vergangenheitsform oder der Plural in einer neuen Sprache gebildet wird. Wenn Kinder (angeleitet aber eigenaktiv) herausfinden, wie die Pluralbildung z.B. im Spanischen funktioniert, werden ihnen auch grammatische Regelmäßigkeiten im Deutschen oder Englischen noch einmal bewusster (vgl. Wolff 2008). Auch beim grammatikalischen Lernen greifen Kinder auf die interlingualen Transferbasen zurück, die ihnen schon beim Erweitern des Lexikons, beim Erschließen von Bedeutungen weiterhelfen:

Der Fremdsprachenlerner als Hypothesentester, der sich seine eigene Grammatik („Spontangrammatik“) entwirft, indem er adhoc-Deutungen verbalisiert, bedient sich permanent solcher Transferbasen. Bereits bekannte Strukturen und Lexeme der erlernten und erworbenen Sprachen werden tentativ mit den neuen Sprachstrukturen 
verbunden. Die Bildung solcher vorläufigen Regularitäten im Kopf des Lerners sollte methodisch im Unterricht fruchtbar gemacht werden, statt sie als unzulänglich zu verwerfen. (Nieweler 2001: 8)

Nicht nur bei einzelnen Begriffen, auch bei ganzen Sätzen, Phrasen oder Redewendungen kann über Gemeinsamkeiten und Unterschiede der Struktur in den verschiedenen Sprachen reflektiert werden. Dies mag bei Formeln oder Kollokationen beginnen, die dem alltäglichen Sprachgebrauch entstammen, wenn z.B. gesammelt wird, wie man sich auf Russisch/ Türkisch/ Englisch/ Italienisch begrüßt oder das Fragewort in den verschiedenen Übersetzungen von „Wie geht es dir? “ herausfiltert. Aber auch bildungssprachliche Ausdrücke, z.B. besonders wichtige Sätze aus anderen Unterrichtsfächern, können thematisiert werden, denn als Wortfeld bietet sich prinzipiell jedes aktuelle Unterrichtsthema an. Sprachvergleiche sind sowohl in den künstlerischen als auch den naturwissenschaftlichen Fächern möglich (vgl. Oomen-Welke 2011).

Als „Sprachendetektive“ gehen Kinder auf Spurensuche nach fremden Sprachen in ihrer Umgebung. Wo finden sich Beschilderungen auch in anderen Sprachen als Deutsch? Warum ist das wichtig? Welche Sprachen finden wir in offiziellen Behörden, am Bahnhof oder Flughafen? Welche Sprachen sind in der Schule repräsentiert? Und warum ist das so? Wenn man mit den Kindern darüber nachdenkt, welche Rolle Sprachen in unserer Gesellschaft spielen, wird die soziale Dimension von Language Awareness gefördert. Hier geht es um das Prestige und die Präsenz verschiedener Sprachen. Die Dominanz des Englischen wird von Kindern schon sehr früh erkannt und es lohnt sich, mit ihnen nach Erklärungen dafür zu suchen, warum eine Sprache wichtiger erscheint als andere. Durch das sprachenübergreifende Arbeiten zur Förderung von Language Awareness wird „neben dem Beschreiben und Vergleichen von sprachlichen Strukturen auch die Akzeptanz für sprachliche Vielfalt geweckt“ (Nieweler 2001: 10).

Es ist hilfreich, Sprachlernstrategien, oder Lese-/Entschlüsselungsstrategien, die für alle Sprachen nützlich sind, sowie bestimmte sprachenübergreifende Phänomene oder eben auch Unterschiede zwischen den Sprachen mit den Kindern zu thematisieren und sie in den konkreten Situationen jeweils zu ermutigen, auf ihr "gesamtes Sprachenrepertoire“ zurückzugreifen (Hufeisen 2008: 389) - statt künstlich Einsprachigkeit zu forcieren. Schulen dürfen nicht das Gefühl vermitteln, einige Sprachen 
müssten ,draußen bleiben', sondern sollten Kinder mit all ihren Sprachen in ihrer gesamten mehrsprachigen Identität willkommen heißen und sie so gut wie möglich in deren Entwicklung unterstützen. Kinder müssen die Erfahrung machen, dass entdeckendes Lernen, d.h. eigenständige, konstruktive Sprachbetrachtung im Unterricht möglich und erlaubt, ja sogar erwünscht ist. Das „willig hörende Ohr der Lehrperson und der MitschülerInnen“ ist dabei eine wichtige Voraussetzung (Oomen-Welke 2011: 50). Eine tiefergehende Forschung in Bezug auf die theoretischen Grundlagen bleibt aber auch weiterhin unverzichtbar „um neue und linguistisch fundierte Wege im Umgang mit Mehrsprachigkeit zu erarbeiten “ (Krafft 2014: 7). Bei der weiteren Erforschung der interlingualen Transferbasen sollte insbesondere der „Aspekt der Produktivität (v.a. Sprechen und Schreiben) stärker in den Blick genommen werden“, Arbeiten zur Grammatik stellen ein weiteres Desiderat der Mehrsprachigkeitsforschung dar (Nieweler 2001: 10).

\section{Literaturverzeichnis}

Atanasoska, Tatjana (2011), Was ist Mehrsprachigkeit?. In: Wilmes, Sabine; Plathner, Franziska \& Atanasoska, Tatjana (Hrsg.) (2011), Second Language Teaching in Multilingual Classes. Basic Principles for Primary Schools. EURAC book 60, 171-181. [Online: http://www.eurac.edu/en/ research/institutes/multilingualism/Publications. 20.05.2015].

Barac, Raluca \& Bialystok, Ellen (2012), Bilingual effects on cognitive and linguistic development: Role of language, cultural background, and education. Child Development 83: 2, 413-422.

Bialystok, Ellen (2001), Metalinguistic aspects of bilingual processing. Annual Review of Applied Linguistics 21, 169-181.

Bialystok, Ellen (2009), Effects of bilingualism on cognitive and linguistic performance across the lifespan. In: Gogolin, Ingrid \& Neumann, Ursula (Hrsg.) (2009), Streitfall Zweisprachigkeit - The Bilingual Controversy. Wiesbaden: VS Verlag für Sozialwissenschaften, 53-67.

Bialystok, Ellen (2011), How Analysis and Control lead to Advantages and Disadvantages in Bilingual Processing. In: Sanz, Christina \& Leow, Ronald P. (Hrsg.) (2011), Implicit and Explicit Language Learning. Conditions, Processes and Knowledge in SLA and Bilingualism. Washington, DC: Georgetown University Press, 49-58. 
Breidbach, Stephan; Elsner, Daniela \& Young, Andrea (Hrsg.) (2011), Language Awareness in Teacher Education, Frankfurt am Main: Peter Lang.

Cenoz, Jasone (2003), The additive effect of bilingualism on third language acquisition: A review. The International Journal of Bilingualism 7: 1, 71-88.

Cummins, Jim (o.J.), ELL students speak for themselves: Identity texts and literacy engagement in multilingual classrooms [Online: http:// learnteachlead.ca/wp-content/uploads/2013/08/ELLidentityTexts.pdf. 22.05.2015].

Cummins, Jim (2008), BICS and CALP: Empirical and theoretical status of the distinction. In: Cenoz, Jasone \& Hornberger, Nancy (Hrsg.) (2008), Encyclopedia of Language and Education (2. Aufl.), Vol. 2: Literacy. New York: Springer, 71-83.

Cummins, Jim (2008), BICS AND CALP: Empirical and Theoretical Status of the Distinction. In: Street, Brian V. \& Hornberger, Nancy (Hrsg.) (2008), Encyclopedia of Language and Education (2. Aufl.), Volume 2: Literacy. New York: Springer, 71-83.

Davidson, Denise \& Raschke, Vanessa (2009), Metalinguistic awareness in monolingual and bilingual children and its relationship to receptive vocabulary scores and performance on reading readiness test. BUCLD 33 Online Proceedings Supplement 01/2009. [Online: http://www.bu.edu/ bucld/files/2011/05/33-Davidson.pdf. 27.02.2015].

Dechert, Hans, W. (1997), Metakognition und Zweitsprachenerwerb. In: Rampillon, Ute \& Zimmermann, Günther (Hrsg.) (1997), Strategien und Techniken beim Erwerb fremder Sprachen. Ismaning: Hueber, 10-32.

Dirim, Inci \& Müller, Astrid (2007): Sprachliche Heterogenität. Deutsch lernen in mehrsprachigen Kontexten. Praxis Deutsch 202/2007, 6-14.

Donmall, Gillian (1985), Language Awareness. NCLE Papers and Reports 6. London.

Doyé, Peter (2004), Intercomprehension. Rezeptive Mehrsprachigkeit als Alternative. Praxis Fremdsprachenunterricht 1/2004, 4-7.

Elsner, Daniela (2010), „Ich habe was, das du nicht hast... “ - oder, welchen Mehrwert hat die Mehrsprachigkeit für das Fremdsprachenlernen? In: Weth, Constanze (Hrsg.) (2010), Schriftspracherwerb unter den Bedingungen von Mehrsprachigkeit und Fremdsprachenunterricht. IMIS Beiträge 37/2010, 99-120. 
Elsner, Daniela (2015), Inklusion von Herkunftssprachen - Mehrsprachigkeit als Herausforderung und Chance. In diesem Band.

Elsner, Daniela \& Lohe, Viviane (2015), Language Awareness. Förderung von Sprachbewusstheit. Grundschulmagazin Englisch 1/2015, 31-34.

Fehling, Sylvia (2008), Language Awareness und bilingualer Unterricht: Eine komparative Studie (2. Aufl.). Frankfurt: Peter Lang.

Fidler, Soca; Avsenik, Bernarda; Velušcek, Andrea German; Šketa, Alma; Jakopica, Riharda; Klavs, Teja; Grad, Katja; Dražumeric, Nataša \& Kumar, Danile (o.J.): Workshop. Language and intercultural awareness in primary school. [online: http://jaling.ecml.at/pdfdocs/IATEFL02SocaFidler.pdf. 21.05.2015].

Friesen, Deanna \& Bialystok, Ellen (2012), Metalinguistic ability in bilingual children: The role of executive control. Rivista di psicolinguistica applicate: RiPLA 12: 3, 47-56.

Galambos, Sylvia Joseph \& Goldin-Meadow, Susan (1990), The effects of learning two languages on levels of metalinguistic awareness. Cognition $34,1-56$.

Gnutzmann, Claus (1997), Multilingualism and language teaching: some pedagogical implications with reference to language awareness. Fremdsprachen lebren und lernen 26, 156-166.

Gogolin, Ingrid (1994), Der monolinguale Habitus der multilingualen Schule. Münster, New York: Waxmann-Verlag (Buchveröffentlichung der Habilitationsschrift) (2. Aufl. 2008).

Hermanto, Nicola; Moreno, Sylvain \& Bialystok, Ellen (2012), Linguistic and metalinguistic outcomes of intense immersion education: How bilingual? International Journal of Bilingual Education and Bilingualism 15: 2, 131-145. [Online: http://www.ncbi.nlm.nih.gov/pmc/articles/ PMC3314398/pdf/nihms363446.pdf. 02.06.2015].

Hohm, Michael (2005), Zum Zusammenhang von Sprachbewusstheit, Lesekompetenz und Textverstehen: Historische, fachdidaktische und unterrichtspraktische Aspekte der Problematik. [Online: http://opus.bibliothek.uni-wuerzburg.de/frontdoor/index/index/docId/1653. 02.06.2015].

Hoti, Haenni; Müller, Marianne; Heinzmann, Sybille; Wicki, Werner \& Werlen, Erika (2009), Frühenglisch- Überforderung oder Chance? Eine Längsschnittstudie zur Wirksamkeit des Fremdsprachenunterrichts auf der Primarstufe. Forschungsbericht Nr. 20 der Pädagogischen Hochschule 
Luzern. [Online: http://www.phlu.ch/fileadmin/media/fe.luzern.phz.ch/

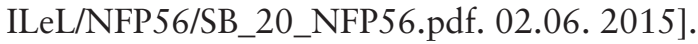

Hufeisen, Britta (2003), L1, L2, L3, L4, Lx - alle gleich? Linguistische, lernerinterne und lernerexterne Faktoren in Modellen zum multiplen Spracherwerb. In: Baumgarten, Nicole; Böttger, Claudia; Motz, Markus \& Probst, Julia (Hrsg.) (2003), Übersetzen, Interkulturelle Kommunikation, Spracherwerb und Sprachvermittlung - das Leben mit mehreren Sprachen. Festschrift für Juliane House zum 60. Geburtstag. Didaktik und Methodik im Bereich Deutsch als Fremdsprache. Zeitschrift für Interkulturellen Fremdsprachenunterricht 8: 2/3, 1-13 [Online: http://zif. spz.tu-darmstadt.de/jg-08-2-3/beitrag/Hufeisen1.htm. 26.02.2015].

Hufeisen, Britta (2008), Lernforschung Deutsch als zweite (und weitere) Sprache. In: Ahrenholz, Bernt \& Oomen-Welke, Ingelore (Hrsg.) (2008), Deutsch als Zweitsprache. (Deutschunterricht in Theorie und Praxis, Handbuch in XI Bänden, hrsg. v. Winfried Ulrich, Bd. 9) Baltmannsweiler: Schneider Verlag Hohengehren, 385-392.

Hufeisen, Britta \& Gibson, Martha (2003), Zur Interdependenz emotionaler und kognitiver Faktoren im Rahmen eines Modells zur Beschreibung sukzessiven multiplen Sprachenlernens. Bulletin VALS-ASLA (Vereinigung für angewandte Linguistik in der Schweiz) 78, 13-33.

Hufeisen, Britta \& Marx, Nicole (2007), EuroComGerm-Die sieben Siebe: Germanische Sprachen lesen lernen. Aachen: Shaker.

Jackson, Daniel O. (2014), Learner differences in metalinguistic awareness: Exploring the influence of cognitive abilities and language experience. In: Miller, Ryan T.; Martin, Katherine I.; Eddington, Chelsea M.; Henery, Ashlie; Marcos Miguel, Nausica; Tseng, Alison M.; Tuninetti, Alba \& Walter, Daniel (Hrsg.) (2014), Selected Proceedings of the 2012 Second Language Research Forum: Building Bridges Between Disciplines. Somerville, MA: Cascadilla Proceedings Project, 211-226.

James, Carl \& Garrett, Peter (1995) (Hrsg.), Language Awareness in the Classroom. London: Longman.

James, Carl \& Garrett, Peter (2002), Language Awareness. In: Byram, Michael (Hrsg.) (2002), Routledge Encyclopedia of Language Teaching and Learning. London/ New York: Routledge, 331-332.

Jessner, Ulrike (2008), State-of-the-art article. Teaching third languages:

Findings, trends and challenges. Language Teaching 41: 1, 15-56. 
Jessner, Ulrike (2009), Linguistic awareness in multilinguals: English as a third language. Edinburgh, UK: Edinburgh University Press.

Jude, Nina (2008), Zur Struktur von Sprachkompetenz. [Online: http:// publikationen.ub.uni-frankfurt.de/volltexte/2009/6794/pdf/Jude Zur Struktur_von_Sprachkompetenz.pdf. 15.01. 2015].

Kirschhock, Eva-Maria (2004), Die Entwicklung schriftsprachlicher Kompetenzen im Anfangsunterricht. Bad Heilbrunn: Klinkhardt.

Knapp-Potthoff, Annelie (1997), Sprach(lern)bewusstheit im Kontext. In: Edmondson, Willis J. \& House, Juliane (Hrsg.) (1997), Themenschwerpunkt: Language Awareness. FLuL 26, 9-23.

Kollmeyer, Katrin (2007), Englischunterricht als Fenster zur Mehrsprachigkeit. In: Elsner, Daniela; Küster, Lutz \& Viebrock, Britta (Hrsg.) (2007), Fremdsprachenkompetenzen für ein wachsendes Europa. Das Leitziel „Multiliteralität“. Frankfurt a.M.: Peter Lang, 256-268.

Krafft, Andreas (2014), Zur Entwicklung metasprachlicher Fähigkeiten bei Kindern mit ein- und mehrsprachigem Hintergrund. Schneider Verlag Hohengehren: Baltmannsweiler.

Lenz, Annina (2009), Fremdsprachenübergreifende Vokabelarbeit im Englischunterricht als Methode zur Förderung von Sprachbewusstheit. Forum Sprache 1: 2, 42-60.

Morkötter, Steffi (2005), Language Awareness und Mehrsprachigkeit. Eine Studie zu Sprachbewusstheit und Mehrsprachigkeit aus der Sicht von Fremdsprachenlernern und Fremdsprachenlehrern. Frankfurt/M.: Lang.

Muñoz, Sandra Bermejo (2014), Implementierung schulischer und lebensweltlicher Mehrsprachigkeit in ein aufgabenorientiertes Unterrichtskonzept im Spanischunterricht der Sekundarstufe II. Zeitschrift für Interkulturellen Fremdsprachenunterricht 19: 1, 119-137 [Online: http://zif.spz. tu-darmstadt.de/jg-19-1/beitrag/Bermejo-,.pdf. 02.06. 2015].

Nicholas, Howard (1995), Language awareness and second language development. In: James, Carl \& Garrett, Peter (Hrsg.) (1995), 78-95.

Nieweler, Andreas (2001), Förderung schulischer Mehrsprachigkeit durch sprachenübergreifendes Unterrichten. In: Abendroth-Timmer, Dagmar \& Bach, Gerhard (Hrsg.) (2001), Mehrsprachiges Europa. Festschrift für Michael Wendt zum 60. Geburtstag. Tübingen: Gunter Narr Verlag, 207-222. 
Oomen-Welke, Ingelore (2003), Entwicklung sprachlichen Wissens und Bewusstseins im mehrsprachigen Kontext. In: Bredel, Ursula; Günther, Hartmut; Klotz, Peter; Ossner, Jakob \& Siebert-Ott, Gesa (Hrsg.) (2003), Didaktik der deutschen Sprache. Paderborn: Schöningh UTB, 452-463.

Oomen-Welke, Ingelore (2011), Sprachen vergleichen auf eigenen Wegen: Der Beitrag des Deutschunterrichts. In: Rothstein, Björn (Hrsg.) (2011), Sprachvergleich in der Schule. Baltmannsweiler: Schneider Hohengehren, 49-70. [Online: faecher.lernnetz.de/faecherportal/index. php?DownloadID=4390. 22.05.2015].

Oomen-Welke, Ingelore \& Pena Schumacher, Tomas (2001): Sprachenlernen -Biographische Rekonstruktionen zweisprachiger Schulkinder. [Online: http://ialing.ecmlat/pdfdocs/articles/IOWbiograph.pdf 09.03.2015].

Portnaia, Natalia (2007), Englischlernen in der Grundschule aus der Sicht von Kindern mit migrationsbedingter Zwei-/Mehrsprachigkeit. In: Elsner, Daniela; Küster, Lutz \& Viebrock, Britta (Hrsg.) (2007), Fremdsprachenkompetenzen für ein wachsendes Europa. Das Leitziel „Multiliteralität“. Frankfurt a.M.: Peter Lang, 107-120.

Rauch, Dominique P.; Naumann, Johannes \& Jude, Nina (2011), Metalinguistic awareness mediates effects of full biliteracy on third-language reading proficiency in Turkish-German bilinguals. International Journal of Bilingualism 16: 4, 402-418.

Riehl, Claudia Maria (2006), Aspekte der Mehrsprachigkeit: Formen, Vorteile, Bedeutung. In: Heints, Detlef; Müller, Jürgen E. \& Reiberg, Ludger (Hrsg.) (2006), Mehrsprachigkeit macht Schule. Duisburg: Gilles \& Francke, 15-23.

Roberts, Anthony David (2011), The Role of Metalinguistic Awareness in the Effective Teaching of Foreign Languages. Oxford u.a.: Springer.

Robinson, Peter (2008), Attention and awareness. In: Cenoz, Jasone \& Hornberger, Nancy (Hrsg.) (2008), Encyclopedia of Language and Education (2. Aufl.), Volume 6: Knowledge about Language. New York: Springer, 133-142.

Schader, Basil (2012), Sprachenvielfalt als Chance: Das Handbuch. Zürich: Bildungsverlag Eins.

Schnuch, Johanna (2014), It's the same in English. Grundschulmagazin Englisch 6, 37-39.

Triarchi-Herrmann, Vassilia (2006), Metalinguistische Fähigkeiten, phonologische Bewusstheit und Rechtschreibfertigkeit von mehrsprachigen 
Kindern mit Migrationshintergrund. Vergleichende Untersuchung bei zweisprachigen griechischen Migrantenkindern. In: Bahr, Reiner \& Iven, Claudia (Hrsg.) (2006), Sprache - Emotion - Bewusstheit Beiträge zur Sprachtherapie in Schule, Praxis, Klinik. Idstein: Schulz-Kirchner, 145-153.

Van Lier, Leo (1995), Introducing Language Awareness. London: Penguin. Wolff, Dieter (1997), Strategien des Textverstehens: Was wissen Fremdsprachenlerner über den eigenen Verstehensprozess? In: Rampillon, Ute \& Zimmermann, Günther (Hrsg.) (1997), Strategien und Techniken beim Erwerb fremder Sprachen. Ismaning: Hueber, 270-289.

Wolff, Dieter (2008), Spracherwerb aus spracherwerbspsychologischer Sicht. Materialien für die Lehrerfortbildung in NRW. Didaktisch-methodische Fortbildung Englisch in der Grundschule NRW. [Online: https://www. standardsicherung.schulministerium.nrw.de/cms/upload/egs/Modul 4 Sprachenlernen_Teil-1.pdf. 20.5.2015].

Wolff, Dieter (2010), Mehrsprachigkeit - Spracherwerb - Sprachbewusstheit: Sind mehrsprachige Menschen bessere Sprachenlerner? In: Gil, Maria Jesús \& Gimber, Arno (Hrsg.) (2010), Sincronías en el pasado... diacronías en el presente: Estudios lingüísticos, didácticos y culturales. Madrid: Publicaciones Universidad Complutense de Madrid, 177-190. 
Christiane M. Bongartz and Andreas Rohde - 978-3-653-97188-0

Downloaded from PubFactory at 01/11/2019 10:41:16AM

via free access 


\section{Jens Boenisch \\ Zur Bedeutung von Kernvokabular im inklusiven Englisch-Anfangsunterricht}

The paper focuses on the development of electronic and non-electronic communication aids for mentally and physically challenged students. To this end, it is vital to identify these students' major communicative needs. Two studies are discussed. The first determines the core vocabulary of mentally challenged and typically developing children, the second study compares the core vocabulary of typically developing native-speakers and second language learners of English. The results of both studies suggest that the communicative needs of challenged and typically developing students are strikingly similar, including the distribution of the different word classes. The author therefore calls for special needs materials that feature the most frequent lexical items as identified in the studies rather than the vocabulary used in textbooks, the objective being electronic and non-electronic communication aids in the shape of tablets and binders that help develop the communicative skills of those students who are not able to use spoken or sign language.

\section{Einführung}

Trotz kontinuierlich steigender Inklusionszahlen werden Kinder mit schweren oder mehrfachen Behinderungen auch weiterhin nur äußerst selten in der Allgemeinen Schule unterrichtet. Neben der pflegerischen Aufgabe, die mit der Aufnahme mehrfachbehinderter Kinder in der Regel einhergeht und die Schulen oft vor große Herausforderungen stellt, kann die komplexe Kommunikationsstörung dieser Schüler als der eigentlich zentrale Grund für den Ausschluss aus dem Allgemeinen Schulsystem angeführt werden. Wenn ein Kind mit körperlicher oder geistiger Behinderung auf Ansprache nicht adäquat reagieren kann, weil ihm z.B. die Fähigkeit fehlt, über Lautsprache verständlich zu kommunizieren, dann verunsichert dies vor allem das soziale Umfeld (Pädagogen, Mitschüler, Eltern nichtbehinderter Kinder). Man meidet die Kontaktaufnahme, man entzieht sich der Interaktion mit der Person, die einen selbst verunsichert. Die Aussonderung von Menschen mit komplexen Behinderungen vollzieht sich in der Folge auf institutioneller Ebene („,So schwer behinderte Kinder können wir hier 
nicht aufnehmen. Das schaffen wir nicht. Dafür sind wir nicht ausgebildet. Dafür sind doch die Förderschulen da!“) oder in den seltenen Fällen der inklusiven Unterrichtung auf sozialer Ebene, solange die Verständigung mit dem behinderten Kind nicht gelingt. Die Frage nach dem Gelingen der Inklusion von Kindern mit schweren Behinderungen ist weniger eine Frage nach baulichen Barrieren, Pflegebedarf oder individuellem Lerntempo als vielmehr eine Frage nach den Möglichkeiten gelingender Kommunikation und Interaktion. In Deutschland ist jedes fünfte Kind an der Förderschule mit dem Förderschwerpunkt körperliche und motorische Entwicklung nichtsprechend, an der Förderschule mit dem Förderschwerpunkt Geistige Entwicklung sogar jedes dritte Kind (vgl. Boenisch 2003, 2009: 126ff.). Seit der Unterzeichnung der Behindertenrechtskonvention (2009) muss die schulische Inklusion auch für diese Schülerschaft einen gleichberechtigten Zugang zu den Bildungsangeboten der Allgemeinen Schule ermöglichen. Die Schwere einer Behinderung darf weder rechtlich noch pädagogisch ein Ausschlusskriterium darstellen, da jedes Kind bildungsfähig ist (vgl. Bernasconi \& Böing 2015). Bildung erfolgt zwar auf unterschiedlichen Niveaustufen und mit unterschiedlichen Vermittlungsmethoden, der Zugang zum Allgemeinen Bildungssystem steht jedoch jedem Schüler im Sinne der Gleichberechtigung unabhängig von Art und Schwere einer Beeinträchtigung zu (vgl. Bergeest, Boenisch \& Daut 2015: 21-58; BRK 2009). Von dieser rechtskräftigen Grundsatzentscheidung im deutschen Bildungssystem ist auch der Englischunterricht betroffen.

Kinder mit Beeinträchtigungen der Kognition, der Motorik, des Sprechens und des Hörens ab der 1. Klasse auch im Englischunterricht inklusiv zu unterrichten, stellt die Didaktik des Fremdsprach-Anfangsunterrichts vor völlig neue Herausforderungen. Wie kann unter den Bedingungen einer schweren Behinderung, insbesondere unter den Bedingungen einer komplexen Kommunikationsstörung ein positiver, motivierender und erfolgsversprechender Zugang zum Zweitspracherwerb ermöglicht werden? Diese „Extremfälle“ erfordern jedoch keine individuellen didaktischen Lösungen, sondern eine neue Sichtweise auf Lern- und Aneignungsprozesse des Erst- und Zweitspracherwerbs, die auch bei weniger schwer und nicht behinderten Kindern Anwendung finden kann. Im Folgenden wird ein in der Unterstützten Kommunikation bereits erfolgreich erprobtes Konzept mit entsprechenden Fördermaterialien, basierend auf neuen 
Forschungsergebnissen zum sogenannten Kernvokabular, vorgestellt und für den inklusiven Englischunterricht adaptiert.

Exkurs: Unterstützte Kommunikation (UK) bezeichnet ein Konzept zur Förderung von Kindern, Jugendlichen und Erwachsenen, die sich aufgrund einer angeborenen oder einer im Laufe des Lebens erworbenen Behinderung nicht oder nicht mehr über Lautsprache verständigen können. In der UK werden sowohl ergänzende Hilfen als auch die Lautsprache ersetzende Hilfen der Verständigung eingesetzt, um die Kommunikation zwischen dem betroffenen Menschen und seinem sozialen Umfeld zu verbessern (z.B. Einsatz von Gestik, Mimik, Gebärden, nicht-elektronischen Kommunikationstafeln/ordnern, elektronischen Kommunikationshilfen / „Sprechcomputer“). UK wird eingesetzt bei Menschen, die Unterstützung beim Spracherwerb und Sprachverständnis benötigen (z.B. geistig behinderte Menschen), die ersetzende Sprechhilfen aufgrund nicht verständlicher Lautsprache benötigen (z.B. bei Menschen mit schweren Formen einer Sprechstörung wie Dysarthrie/Anarthrie) sowie bei Menschen, die aufgrund von Operationen im Artikulationsbereich übergangsweise alternative Sprechhilfen benötigen (vgl. Boenisch 2009: 15; Braun 2008). Im anglo-amerikanischen Sprachraum ist das Konzept der UK unter dem Begriff AAC (Augmentative and Alternative Communication) bekannt.

\section{Kernvokabular}

Der Begriff Kernvokabular (core vocabulary) hat sich international als Fachbegriff für die am häufigsten gesprochenen Wörter der Alltagssprache etabliert. Obwohl einige Studien aufgrund unterschiedlicher linguistischer Analyseverfahren und abweichender Populationsgruppen (Vorschulkinder, Schulkinder mit und ohne Behinderung, Jugendliche, Erwachsene) keinen eindeutigen Wortschatz als Kernvokabular bestimmen können, so zeigen die Studien dennoch vergleichbare Ergebnisse. Übereinstimmend kann man zusammenfassen, dass die 200-250 am häufigsten gesprochenen Wörter ca. 80-85\% der Alltagssprache ausmachen - und zwar unabhängig von Alter, kognitiver oder motorischer Beeinträchtigung (vgl. Banajee, Dicarlo \& Stricklin 2003; Beukelman, Jones \& Rowan 1989; Boenisch 2014; Boenisch \& Sachse 2007; Clendon \& Erickson 2008; Trembath, Balandin \& Togher 2007). Das Kernvokabular besteht vor 
allem aus sogenannten kleinen Wörtern (Funktionswörtern) wie ich, $d u$, wir, haben, können, wollen, auch, nicht, kein, noch mal, das, weg, hier, dort, auf etc. Demgegenüber steht das Randvokabular (fringe vocabulary), das überwiegend aus Inhaltswörtern wie Auto, Puppe, Computer, spielen, laufen, singen, fleißig, langweilig etc. besteht. Es macht zwar den größten Teil unseres Wortschatzes aus, aber vier Fünftel unserer Alltagssprache besteht aus Kernvokabular.

\section{Abb. 1: Kern- und Randvokabular}

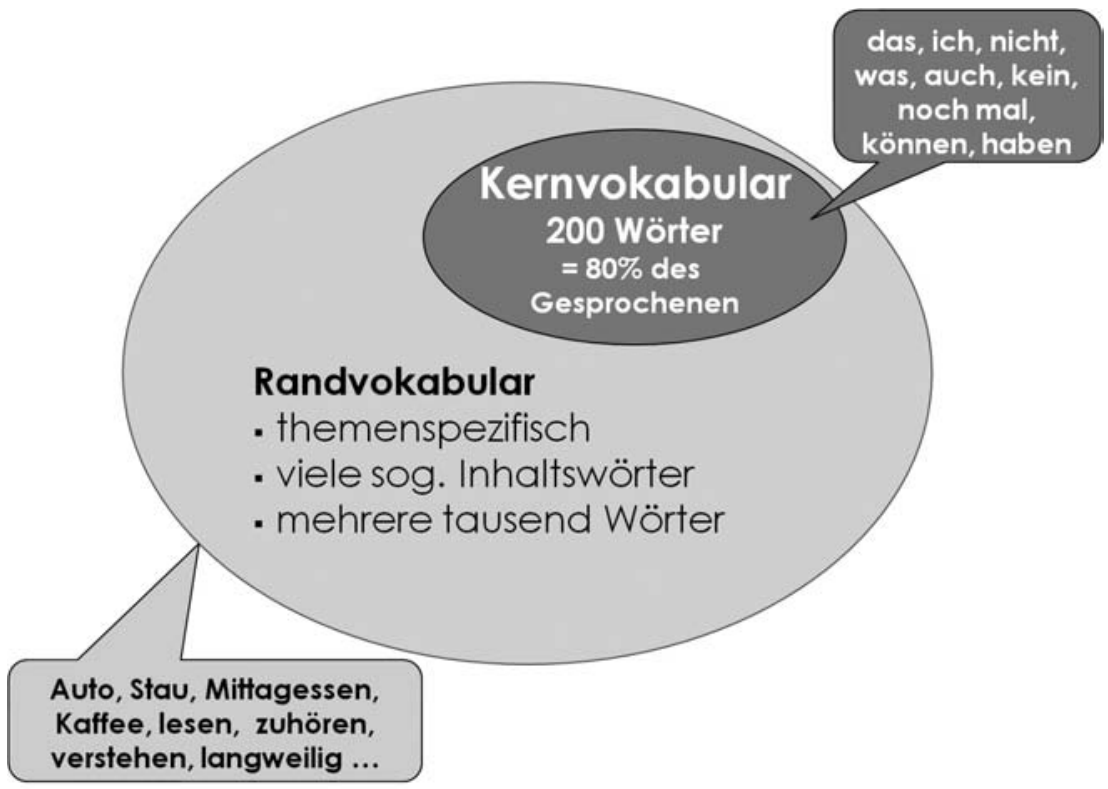

Aufgrund der bisherigen Forschungsergebnisse wurde von Boenisch (2014: 10) folgende Definition vorgeschlagen:

Kernvokabular bezeichnet die am häufigsten verwendeten Wörter einer Sprache. Das Kernvokabular macht $80 \%$ des Gesprochenen aus und wird unabhängig von der individuellen Lebenssituation und vom Thema flexibel eingesetzt. Es sind vor allem situationsunspezifische Funktionswörter (Pronomen, Hilfsverben, Adverbien, Präpositionen, Artikel, Konjunktionen), die durch einzelne Inhaltswörter (Nomen, Verben, Adjektive) ergänzt werden. 
Die 80\%-Marke ist eine relativ leicht zu identifizierende Größe in der Analyse von Wortschätzen und kann altersübergreifend angewandt werden. Damit wird Pädagogen und Therapeuten in unterschiedlichen Arbeitsfeldern die Möglichkeit eröffnet, mit Hilfe von Häufigkeitswortlisten gesprochener Sprache schnell und zuverlässig das Kernvokabular der Bezugsgruppe bzw. der zu erlernenden Sprache zu identifizieren. Zu beachten ist jedoch, dass es deutliche Unterschiede zwischen der gesprochenen Sprache und der Schriftsprache gibt. Schriftsprachbasierte Wortlisten sind als Basis für die Entwicklung von didaktischen Sprachfördermaterialien nur bedingt geeignet.

\section{Forschungsergebnisse zum Kernvokabular von Kindern und Jugendlichen (Deutsch/Englisch)}

Aufbauend auf die nicht nur national, sondern auch international überwiegend im Vorschulbereich durchgeführten Kernvokabularstudien wurden im Kölner Forschungs- und Beratungszentrum für Unterstützte Kommunikation (FBZ-UK) zwei Studien zum Kernvokabular von a) Schülern an Allgemeinen Schulen im Vergleich zu Schülern mit geistiger Behinderung in Deutschland und b) Schülern mit Englisch als Erstsprache im Vergleich zu Schülern mit Englisch als Zweitsprache in Kalifornien (USA) durchgeführt. Ziel der Untersuchungen war es, Gemeinsamkeiten und Unterschiede im Kernvokabular sowohl zwischen Schülern mit und ohne geistiger Behinderung herauszufinden als auch zwischen Schülern, die Englisch als Erstsprache erworben haben zu denen, die sich mitten im Prozess der Aneignung von Englisch als Zweitsprache befinden. Beide Studien werden zunächst kurz skizziert, bevor im Anschluss ausgewählte Ergebnisse dargestellt und vergleichend aufeinander bezogen werden. Ausführlich sind die beiden Studien jeweils publiziert unter Boenisch (2014) und Boenisch \& Soto (2015).

\section{Kölner Studie}

Die Kölner Studie wurde zwischen 2010 und 2012 an unterschiedlichen Kölner Schulen durchgeführt und hat folgende Schülergruppen umfasst:

a) Klasse 2, Grundschule: 13 Schüler

b) Klasse 4, Grundschule: 15 Schüler

c) Klasse 6, Hauptschule: 11 Schüler 
d) Klasse 8, Hauptschule: 10 Schüler

e) Klasse 6, Gymnasium: 9 Schüler

f) Schulbesuchsjahr 2-4, Förderschule Geistige Entwicklung: 16 Schüler

g) Schulbesuchsjahr 5-10, Förderschule Geistige Entwicklung: 28 Schüler

Die Wortschatzerfassung erfolgte mit Hilfe von mobilen Audioaufnahmegeräten (Sony IC Recorder), die die Schüler in ihren Taschen trugen. Über ein am Kragen befestigtes Mikrophon wurde die Sprache des jeweiligen Kindes aufgenommen.

\section{Ablauf}

Nach Abschluss der entsprechenden Genehmigungsverfahren wurde im laufenden Schulbetrieb die Alltagssprache der Schüler aufgenommen. Die Äußerungen der ausgewählten Schüler wurden transkribiert, Äußerungen Dritter oder zufällig aufgenommene Gespräche Dritter wurden nicht verschriftlicht.

Die Sprachaufnahmen vollzogen sich in unterschiedlichen Kontexten der Schüler (Unterricht, Pause, Offene Ganztagsschule/Hort, Ausflug, Essen). Ziel der Aufnahmen war es, ein möglichst breites Spektrum an kommunikativen Situationen im Schulalltag zu erfassen.

Die Transkripte wurden anonymisiert und mit der linguistischen Software LingoFox $@$ ausgewertet. Unterschiedliche Erscheinungsformen der Wörter wurden zunächst differenziert aufgelistet, um die unterschiedlichen Wortformen im Anschluss in eine einheitliche Grundform umzuwandeln (habe, hab, hatte, gehabt, hatten $\rightarrow$ haben; laufen, lief, gelaufen, lauf $\rightarrow$ laufen; schnell, schneller, am schnellsten $\rightarrow$ schnell; grüne, grüner, grünes $\rightarrow$ grün etc.). Bei nicht eindeutigen Wörtern erfolgte eine nachträgliche Überprüfung über eine Kontextanalyse. Zusammengesetzte Verben wurden bei Bedarf in die Grundform transformiert, ohne sie auseinander zu reißen (weggelaufen $\rightarrow$ weglaufen). Ferner wurden Substantivierungen über Kontextanalysen identifiziert und zur Grundform verändert (die Schönste, am Größten, Radfahren etc.). Nach Bereinigung, Vereinheitlichung und Zuordnung aller mehrdeutigen, besonderen, konjugier- oder deklinierbaren Wörter erfolgte die Auflistung der Wörter nach Häufigkeit (Ranking). Die Häufigkeitsanalysen differenzieren sich dabei nach unterschiedlichen Kriterien. So wurden zunächst die Gruppen einzeln gelistet (Kl. 2, Kl. 4, Kl. 6 Hauptschule etc.) 
und miteinander verglichen. Es wurden aber auch Gruppen zusammengefasst und mit anderen Gruppen verglichen (z.B. Kl. 2 mit Schülern aus dem Jahrgang 2-4 der Förderschule Geistige Entwicklung), so dass am Ende differenzierte und aussagekräftige Ergebnisse vorgelegt werden können.

\section{Ergebnisse}

Mit den Aufnahmen a) - g) konnten von 102 Schülern insgesamt ca. 260.000 Wörter erfasst und analysiert werden. Im Durchschnitt wurden von jedem nichtbehinderten Schüler 2.300 Wörter und von jedem Schüler mit geistiger Behinderung ca. 3.200 Wörter ausgewertet. Die 58 nicht beeinträchtigten Kinder und Jugendlichen aus den Allgemeinen Schulen (a-e) haben insgesamt 133.453 Wörter gesprochen. Die Sprachanalysen der 44 Schüler mit geistiger Behinderung ( $\mathrm{f}-\mathrm{g}$ ) ergaben eine Gesamtzahl von 125.454 Wörtern.

\section{Kernvokabular im Vergleich}

Mit Blick auf die in Abschnitt 1 formulierte Definition von Kernvokabular besteht zunächst die Analyseaufgabe darin, die 80\%-Marke in den vorliegenden Wortlisten zu identifizieren, um die Wörter der jeweiligen Schülergruppen bis zu dieser Marke zu vergleichen.

Folgende Tabelle zeigt die 80\%-Marke der jeweiligen Schülergruppen. Zur besseren Vergleichbarkeit mit der Gruppe der geistig behinderten Schüler wurde der Korpus der nicht behinderten Schüler der Klasse 2-8 um ein paar Transkripte reduziert, um auf ca. 125.000 Wörter zu kommen.

Tabelle 1: $80 \%$-Marke des Kernvokabulars

\begin{tabular}{|l|c|c|c|c|c|}
\hline & $\begin{array}{c}\text { K1. 2-8 } \\
\text { reduziert } \\
(\mathrm{n}=125.607)\end{array}$ & $\begin{array}{c}\text { GB } \\
\text { gesamt }\end{array}$ & $\begin{array}{c}\text { GS } \\
\text { K1.2 } \\
(\mathrm{n}=125.454)\end{array}$ & $\begin{array}{c}\text { HS } \\
\text { K1. 6+8 }\end{array}$ & $\begin{array}{c}\text { Gym } \\
\text { K1. 6 } \\
(\mathrm{n}=7.726)\end{array}$ \\
\hline $\begin{array}{l}\text { 80\%-Marke bei } \\
\text { Listenplatz... }\end{array}$ & 211 & 201 & 181 & 195 & 158 \\
\hline
\end{tabular}

Dass die 80\%-Marke keine feste Größe in Wortlisten darstellt, sondern in der Regel auch vom Umfang des analysierten Wortkorpus abhängt, wird durch den Vergleich der Schülergruppen deutlich. Je kleiner der erfasste Wortkorpus der Alltagssprache ist, desto niedriger liegt die 80\%-Marke. 


\section{Gemeinsamkeiten und Unterschiede in den Rangfolgen}

Ausgehend vom erfassten Wortschatz der Schüler mit geistiger Behinderung wird mit der folgenden Analyse beispielhaft aufgezeigt, wie gering die Unterschiede im Alltagssprachgebrauch dieser Population zu nicht behinderten Kindern sind. Als Vergleichsgröße wird hier der erfasste Wortschatz der Klasse 2 (Schulalter: 7-8 Jahre) herangezogen, da regelhaft entwickelte Kinder den Spracherwerb mit 4-6 Jahren weitgehend abgeschlossen und ab 8 Jahren sogar über ein explizites Sprachwissen verfügen, also über ihre Sprache reflektieren und sprachliche Verbesserungen begründen können (vgl. Grimm \& Weinert 2002: 534f.). Gleichzeitig erweitern Kinder in diesem Alter ständig ihren Wortschatz; ihre Ausdrucksfähigkeit wird vielfältiger und differenzierter.

Abb. 2: Prozentualer Anteil der gemeinsamen Wörter in den Häufigkeitslisten der Schüler mit geistiger Behinderung (GB gesamt) im Vergleich zu den Schülern der Klasse 2 (GS Kl. 2)

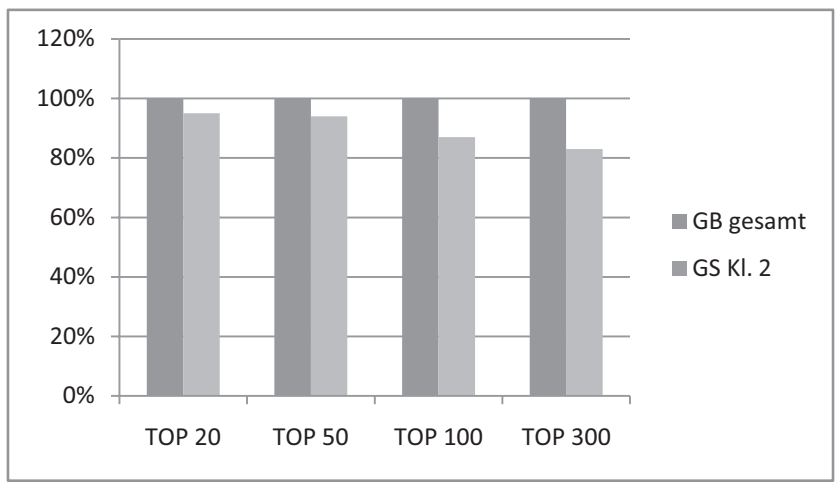

Auffällig ist die hohe Übereinstimmung in den Top 20-Top 300 Listen zwischen den Schülern mit geistiger Behinderung und den Schülern der Grundschulklasse 2. So fehlt beispielsweise nur das Wort auch in der Top 20 Liste der Klasse 2. Dies findet sich allerdings bereits auf Platz 21 wieder. In der Top 50 Listen fehlen die drei Begriffe zu, denn und Hallo, die wiederum in der Top 100 Liste der Klasse 2 auftauchen. Die 13 fehlenden Begriffe in der Top 100 Liste tauchen in der Top 300 Liste der Klasse 2 auf. Größere Abweichungen in den Wortlisten sind erst nach den 200 am häufigsten 
genutzten Wörtern erkennbar. Dies wird verständlich, wenn man die Häufigkeit dieser abweichenden Wörter in den TOP 300 Listen analysiert. Die absoluten Häufigkeiten der Wörter ab den TOP 300 sind im Verhältnis aller gesprochenen Wörter auf ein so niedriges Niveau abgesunken, dass die Wahrscheinlichkeit, ein häufiges Wort des Alltags jenseits der TOP 300 zu finden, sehr gering ist (vgl. Abb. 4).

Ebenso lassen sich kaum Unterschiede im Vergleich einzelner Schülergruppen wie z.B. der Klasse 2 und Klasse 6 Gymnasium finden. Diese Analysen belegen in Ergänzung zu den internationalen Studien noch einmal nachdrücklich, dass das weitgehend gleiche Kernvokabular unabhängig von Alter und Bildungsstand eingesetzt wird und eine lebens-, situationsund behinderungsübergreifende Funktion in der Alltagssprache einnimmt.

\section{Verteilung einzelner Wortarten und verschiedener Wörter (NDW)}

Da in unterschiedlichen Publikationen zu Sprachdiagnostik und Sprachförderung sprachentwicklungsverzögerter bzw. (geistig) behinderter Kinder immer wieder die hohe Relevanz der Wortschatzförderung mit Fokus auf Nomen und Verben betont wird (vgl. u.a. Aktas 2012), diese Inhaltswortdominanz aber auch in den verbreiteten Englischlehrwerken der Klasse 1 und 2 auffällig ist und sich diese inhaltswort-orientierte Sprachförderung als Prinzip auch bis in die Erwachsenensprachförderung durchzieht, wird im Folgenden eine Analyse der Wortarten und der Häufigkeitsverteilung vorgenommen, um die Relevanz dieses tradierten Prinzips in der Sprachförderung zu überprüfen.

Wie in Abb. 3 dargestellt, haben die Kinder ohne Behinderung im Alter von 8-14 Jahren von den insgesamt 133.453 gesprochenen Wörtern zu einem Viertel Pronomen (24,6\%) eingesetzt. Ein Anteil von jeweils einem Zehntel fällt auf Hilfsverben (10,7\%), (Voll-) Verben (12,2\%), Nomen $(10,2 \%)$ und Adverbien (10,7\%). Konjunktionen wurden mit einer Häufigkeit von 7,6\% und Präpositionen mit 2,9\% verwendet. (Im weiteren Verlauf des Textes bezieht sich der Begriff Verb immer auf Vollverben!). Interessanterweise lassen sich keine signifikanten Unterschiede in der Verteilung der Wortarten bei den Kindern und Jugendlichen mit geistiger Behinderung (125.454 Wörter) feststellen. Mit maximal 1\% weicht der Anteil von der Verteilung der Kinder ohne Behinderung ab (vgl. Abb. 3). 
Abb. 3: Prozentualer Anteil der Wortarten gesprochener Sprache von Schülern der Klassen 2, 4, 6 und 8 (Grundschule, Hauptschule, Gymnasium; $n=133.453$ Wörter) im Vergleich zur Gruppe der Schüler mit geistiger Behinderung (2.-10. Schulbesuchsjahr, $n=125.454$ Wörter)

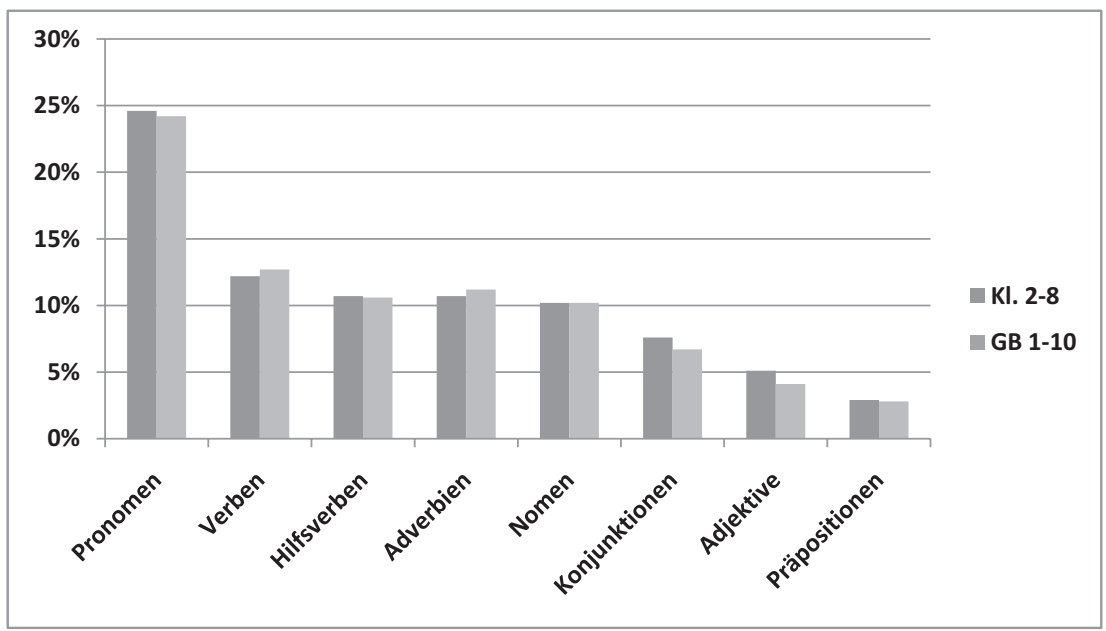

Neben der identischen Wortartenverteilung zwischen beiden Schülergruppen stellt Abb. 3 auch die Bedeutung der Pronomen im Vergleich zu den anderen Wortarten deutlich heraus. Abb. 3 veranschaulicht ferner, dass der Anteil an Hilfsverben sogar noch etwas höher ist als der Anteil an Nomen. D.h. die Pronomen und die Hilfsverben stechen in ihrer Häufigkeit der Nutzung und somit in der Bedeutung für die Alltagssprache in besonderer Weise hervor.

Analysiert man die Verben und Hilfsverben genauer, so erstaunt die Tatsache, dass letztlich die neun Hilfsverben wollen, machen, sollen, mögen, können, sein, müssen, dürfen und haben mit einem Anteil von 10\%-11\% fast genauso häufig gesprochen worden sind wie alle anderen (Voll-)Verben der jeweiligen Schülergruppe zusammen (11\%-12\%). Eine weitere Analyse dieser beiden Wortarten zeigt, dass den neun verschiedenen Hilfsverben insgesamt 1.232 verschiedene Verben gegenüber stehen (Korpus: Klasse 2-8).

\section{Frequenz der Top 300}

In beiden Ranglisten steht das Wort Ich auf Platz 1. Von den nicht behinderten Schülern wird Ich insgesamt 6.549-mal gesprochen. Gleich steht 
auf Platz 100 und konnte nur noch 192-mal gezählt werden. Auf dem Rangplatz 300 stehen insgesamt drei Wörter (trotzdem, Geld, Mal). Diese Wörter wurden nur noch 43-mal geäußert. Bei der Schülergruppe aus der Förderschule Geistige Entwicklung ist der Verlauf identisch: Ich wurde 6.409-mal gesprochen, dies steht auf Platz 100 und wurde 194-mal geäußert. Dem Platz 300 konnten sieben Begriffe zugeordnet werden, die in den Transkripten insgesamt je 45-mal aufgetreten sind (Bruder, egal, elf, hoch, ihn, kaufen, suchen). Abb. 3 verdeutlicht die klare Übereinstimmung in der Häufigkeitsverteilung der TOP 500 Wörter zwischen den beiden Vergleichsgruppen.

Abb. 4: Absolute Hänfigkeiten der TOP 300 Wörter der Schüler aus der

Förderschule Geistige Entwicklung (Schulbesuchsjahr 2-10, $n=125.454$

Wörter; graue Kurve) und der nicht behinderten Schüler aus Klasse 2-8 ( $n=125.607$ Wörter, schwarze Kurve)

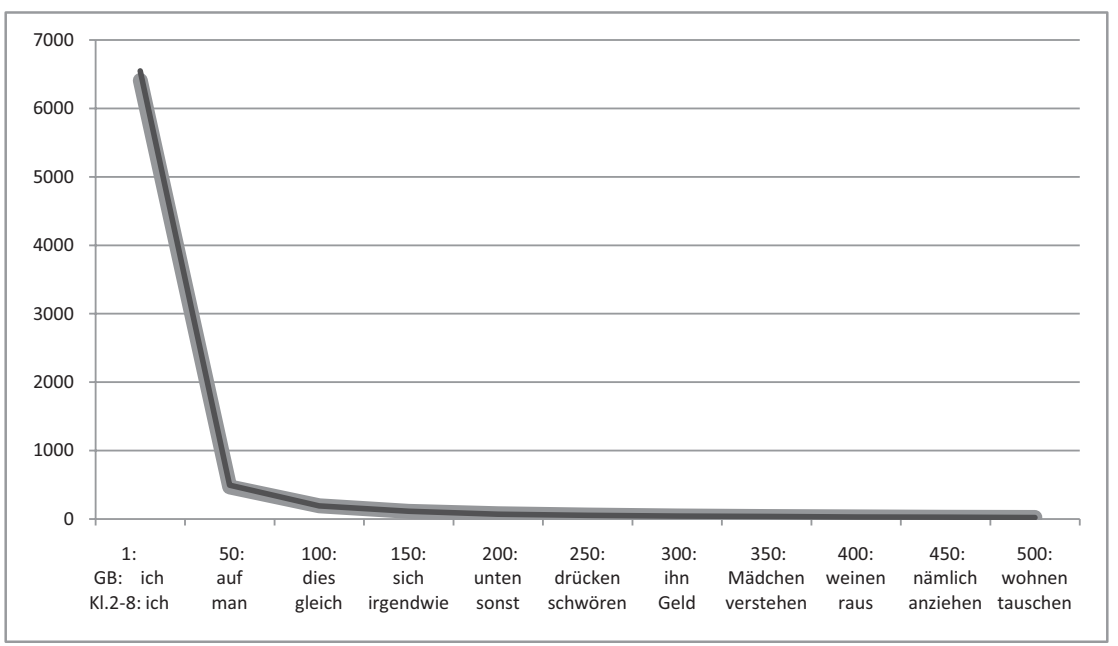

Im Ergebnis heißt das, dass trotz der großen kognitiven Unterschiede und der vielfach diagnostizierten Sprachunterschiede zwischen geistig behinderten und nicht behinderten Kindern und Jugendlichen in der Verlaufskurve der am häufigsten gesprochenen Wörter keine Abweichungen festzustellen sind. Die besondere Bedeutung der Top 100 Wörter, die ganz wesentlich unsere Alltagssprache charakterisieren, wird durch folgendes Ergebnis bekräftigt: Die 32 am häufigsten gesprochenen Wörter der Kinder mit geistiger 
Behinderung machen in dieser Erhebung bereits 50\% des Gesamtkorpus aus. Bei den nicht behinderten Kindern liegt die 50\%-Marke mit 34 Wörtern nur geringfügig höher. Die 100 am häufigsten gesprochenen Wörter machen in beiden Gruppen 70\% des insgesamt erfassten Wortschatzes aus. Von über 125.000 gesprochenen Wörtern treten die Wörter jenseits der Top 200 seltener als 80-mal, jenseits der Top 300 seltener als 50-mal und jenseits der Top 500 seltener als 25-mal auf. Anders formuliert bedeutet dies, dass die Wahrscheinlichkeit des Auftretens der Wörter zwischen den Top 200 und den Top 500 bei nur noch 0,061\% - 0,020\% liegt.

Die bisher vorgestellten Zahlen sollten nicht dazu verleiten zu denken, dass die Wörter jenseits der Top 200-Marke für die Kommunikation unbedeutend sind. Dass der Anteil an Nomen und Verben mit 74\% (K1. 2-8) bzw. 77\% (GB gesamt) drei Viertel aller verschiedenen Wörter (NDW) umfasst, obwohl Nomen und Verben nur einen Anteil von ca. 22\% am Gesamtwortschatz einnehmen, verweist noch einmal auf die Vielfalt dieser Inhaltswörter. Und diese Vielfalt ist es, die uns die Möglichkeit gibt, in einer konkreten Situation mit den richtigen Begriffen einer Verständigung, einem Streit, einem Gespräch oder einem Wunsch die ausschlaggebende Richtung zu geben. Jedoch ist angesichts der hohen kommunikativen Funktion des Kernvokabulars (Top 200) zu hinterfragen, inwiefern dieses Vokabular nicht eine herausgehobene Stellung in der Vermittlung einer (Fremd-) Sprache erhalten sollte, da ihm offensichtlich ein universaler, behinderungs- und altersunabhängiger Charakter innewohnt.

Weitere Analysen (vgl. Boenisch 2014) konnten ferner verdeutlichen, dass es nicht ausreicht, sich nur auf die Verteilung der Wortarten zu fokussieren, um eine Aussage zur Sprachförderung im Bereich Wortschatz machen zu können. So zeigt eine Analyse zu den verschiedenen Wörtern innerhalb der Wortarten, dass innerhalb der Top 200 Wörter zwar die Funktionswörter eine sehr hohe Relevanz haben, der prozentuale Anteil der Inhaltswörter jedoch ab der 200-Wörter-Marke sukzessiv ansteigt. Sprachliche Eloquenz beginnt mit der Zunahme gerade dieser Wörter. Da die sprachliche Kompetenz ein entscheidender Indikator für die kognitive Entwicklung ist, gilt es, diesen Bereich des Wortschatzes in der Sprachförderung immer mit zu bedenken.

Trotz der deutlichen Veränderung in der Wortartenverteilung jenseits der Top 200 Wörter bleibt zusammenfassend festzuhalten, dass die vielerorts postulierte Fokussierung auf Inhaltswörter in der frühen Sprachförderung 
durch die hier vorliegenden Studien nicht bestätig werden kann. Im Gegenteil, um Kinder früh an der Kommunikation teilhaben zu lassen, ist zu Beginn der Sprachförderung eine Fokussierung auf das flexibel einsetzbare Kernvokabular für den Kommunikationserfolg deutlich effektiver. Wenn sich dieses Resultat auch in der englischen Sprache bestätigen sollte, hätte dies umfassende Konsequenzen sowohl auf die Sprachförderung kommunikationsbeeinträchtigter Kinder als auch auf den Anfangsunterricht in Englisch (s. Kap. 5).

\section{Kalifornische Studie}

Methodisch genauso aufgebaut wie die Kölner Studie wurde nach entsprechenden Genehmigungsverfahren im Winter 2011/12 im kalifornischen Berkeley (San Francisco Bay Area) die Alltagssprache von insgesamt 30 Schülern aus den Klassen 2, 4, 6 und 8 aufgenommen. 20 Schüler besuchten die Elementary School, zehn Schüler gingen auf die Middle School. 22 Schüler waren Native Speaker und 8 Schüler waren ESL-Schüler in entsprechenden ESL-Klassen (English as a Second Language). Insgesamt wurden ca. 65 Stunden Sprachaufnahmen transkribiert und linguistisch mit LingoFox ( analysiert, nachbearbeitet und den entsprechenden Wortarten zugeordnet. Es konnten 98.053 Wörter von den Native Speakers und 19.318 Wörter von den ESL-Schülern erfasst und ausgewertet werden. Zur besseren Vergleichbarkeit mit den ESL-Schülern wurde der Korpus der Native Speaker um einige Samples reduziert, so dass hier ein vergleichbarer Korpus mit ca. 19.000 Wörtern entstanden ist. Die Tabelle 2 verdeutlicht, dass bei allen drei Korpora die Top 100 Wörter bereits mehr als 70\% und die Top 300 Wörter sogar zwischen $85-89 \%$ des gesamten Redeanteils ausmachen.

Tabelle 2: Anteilige Wörterverteilung bei Native Speakers und ESL-Schülern in den Top 100, 200 und 300

\begin{tabular}{|l|l|l|l|}
\hline & Native Speaker & Native Speaker & ESL Speaker \\
\hline & $n=27$ samples & $n=10$ samples & $n=10$ samples \\
\hline Total number of words & 98,053 & 19,885 & 19,318 \\
\hline Top 100 words & $69,791(71.2 \%)$ & $14,442(72.6 \%)$ & $14,460(74.8 \%)$ \\
\hline Top 200 words & $78,719(80.3 \%)$ & $16,377(82.4 \%)$ & $16,331(84.5 \%)$ \\
\hline Top 300 words & $83,454(85.1 \%)$ & $17,436(87.7 \%)$ & $17,273(89.4 \%)$ \\
\hline
\end{tabular}


Die Abbildung 5 verdeutlicht darüber hinaus, dass vergleichbar zur deutschen Studie die Frequenz des Kernvokabulars der Native Speaker identisch verläuft zur Frequenz des Kernvokabulars der ESL-Schüler. Da auch bei den Top 20, 50 und 100 Wörtern kaum Unterschiede in der Vokabularauswahl bestehen (vgl. Boenisch \& Soto 2015), bestätigt auch diese Studie die hohe Bedeutung des weitgehend konstanten Kernvokabulars in der englischen Sprache. Diese Erkenntnis wird zusätzlich untermauert durch eine kanadische Vergleichsstudie zum monolingualen und bilingualen Spracherwerb von Kindern mit und ohne Behinderung (Robillard, Mayer-Crittenden, Minor-Corriveau \& Bélanger 2014).

Abb. 5: Frequenz der Top 300 Wörter von Native Speaker (n=10) im Vergleich zu ESL-Schülern ( $n=10)$

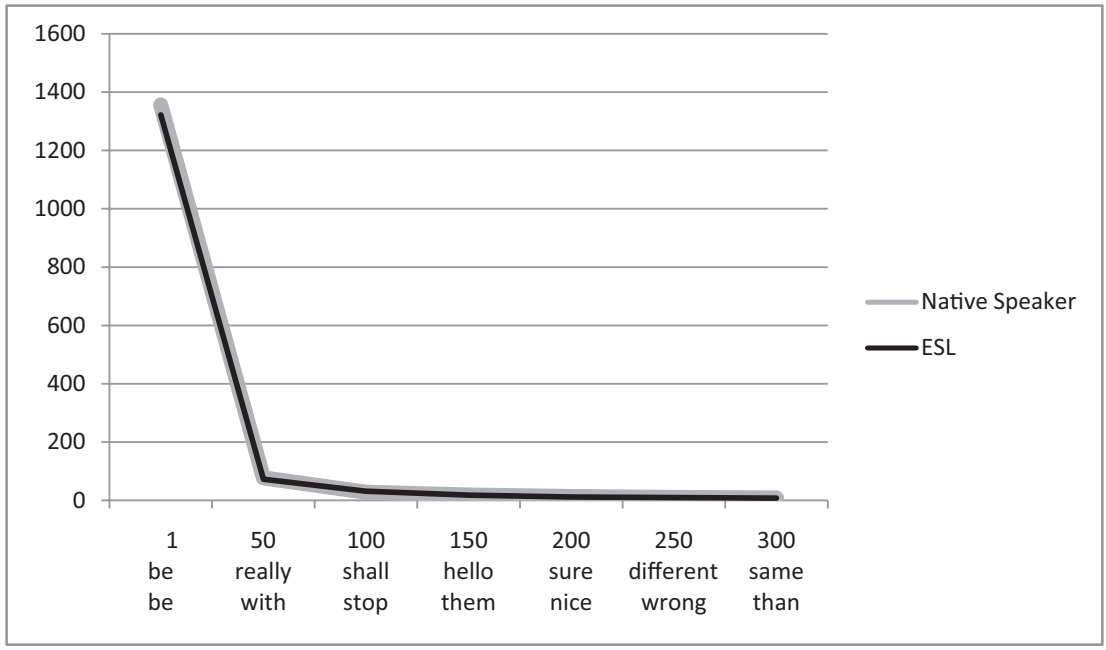

Trotz der kulturellen und sprachlichen Unterschiede sind nicht nur die Wortfrequenzkurven zum Kernvokabular zwischen den deutschen und englischen Schülern - unabhängig von Behinderung und vertiefenden Fremdsprachkenntnissen - vergleichbar, sondern auch ein Großteil der Wörter in der Top 100 Liste (vgl. Anhang). Vor dem Hintergrund der linguistischen Besonderheit, dass im Gegenteil zum Deutschen im Englischen die Adverbien nicht zu den Funktionswörtern, sondern zu den Inhaltswörtern gezählt werden, bestätigt das Resümee von Boenisch \& Soto (2015: 81) in besonderer Weise 
die hier in aller Kürze vorgestellten Kölner und Kalifornischen Studienergebnisse als grundlegend für den Gebrauch der Alltagssprache:

In our top 100 words, only $39 \%$ are content words: $15 \%$ are verbs, $13 \%$ are adverbs, $4 \%$ are adjectives, and $7 \%$ are nouns. The proportion of function words decreases as more words are considered into the analysis. This could be explained by the fact that function words belong to closed word classes and therefore there are a limited number of them. Most function words were among the top 100 for both [native \& ESL speakers; J.B.] groups. (Boenisch \& Soto 2015: 81)

\section{Pädagogische Konsequenzen}

Vor dem Hintergrund dieser Studienergebnisse wurden im FBZ-UK der Universität zu Köln Sprachfördermaterialien entwickelt, die Kindern mit komplexen Kommunikationsstörungen und kognitiven Beeinträchtigungen anhand von Kernvokabular basierten Kommunikationshilfen einen Zugang zur schnellen und flexiblen Kommunikation im Alltag ermöglichen. Die deutschen Materialien wurden 2007 erstmalig auf einer UK-Fachtagung vorgestellt. Aufgrund der hohen Nachfrage und der positiven Rückmeldungen aus der Praxis zum Effekt des Einsatzes von Kernvokabular in der Sprachförderung bei geistig behinderten und bei komplex kommunikationsbeeinträchtigten Kindern wurden die Fördermaterialien seitdem weiter ausdifferenziert, ergänzend zu den nicht-elektronischen Hilfen wurde die Kernvokabular basierte elektronische Kommunikationshilfe MyCore entwickelt (vgl. Sachse, Schmidt \& Wagter 2013) sowie ein völlig neues Sprachförderkonzept zur Förderung des Wortschatzes und der Kommunikationsfähigkeit (vgl. Sachse \& Boenisch 2009; Sachse \& Willke 2011). Vor dem Hintergrund der bisherigen Erfahrungen mit den deutschen Materialien und auf der Basis der englischen Ergebnisse sind neue englische Sprachfördermaterialien und eine englischsprachige Version der elektronischen Kommunikationshilfe MyCore entstanden. Da die unterstützt kommunizierenden Kinder aufgrund des Alters oder der Schwere der Behinderung in der Regel nicht über Schriftsprachkenntnisse verfügen, sind die Sprachfördermaterialien in Form symbolbasierter Kommunikationsoberflächen konzipiert. Dies vereinfacht die Wiedererkennung und führt zu einer schnellen Kommunikation, da jedes Symbol ein ganzes Wort darstellt und so nicht einzelne Buchstaben nach und nach aneinander gereiht werden müssen. Dieses Prinzip lässt sich auch auf den Englisch-Anfangsunterricht übertragen. 
Die Kinder lernen mit symbolbasierten und auf Kernvokabular fokussierten Sprachfördermaterialien, sich mit relativ wenigen Begriffen schnell und flexibel ausdrücken zu können und gewinnen so Spaß und Freude, sich in einer Fremdsprache zu verständigen. Die in Abb. 6 dargestellte SymbolKlett-Tafel ermöglicht den unterstützt kommunizierenden wie den nicht behinderten Schülern, ihre Aussagen nicht nur mündlich zu tätigen, sondern unter Anwendung der verschiedenen Wortarten (hier farblich geordnet nach dem Montessori-Sprachförderkonzept) in zielsprachlich korrekter Grammatik für sich und alle Mitschüler sichtbar zusammen zu stellen. Die neue Sprache wird nicht nur auditiv, sondern auch visuell dargestellt und somit schneller verständlich.

Abb. 6: Kernvokabularbasierte englische Kommunikations-Wandtafel (Symbole (C) METACOM)

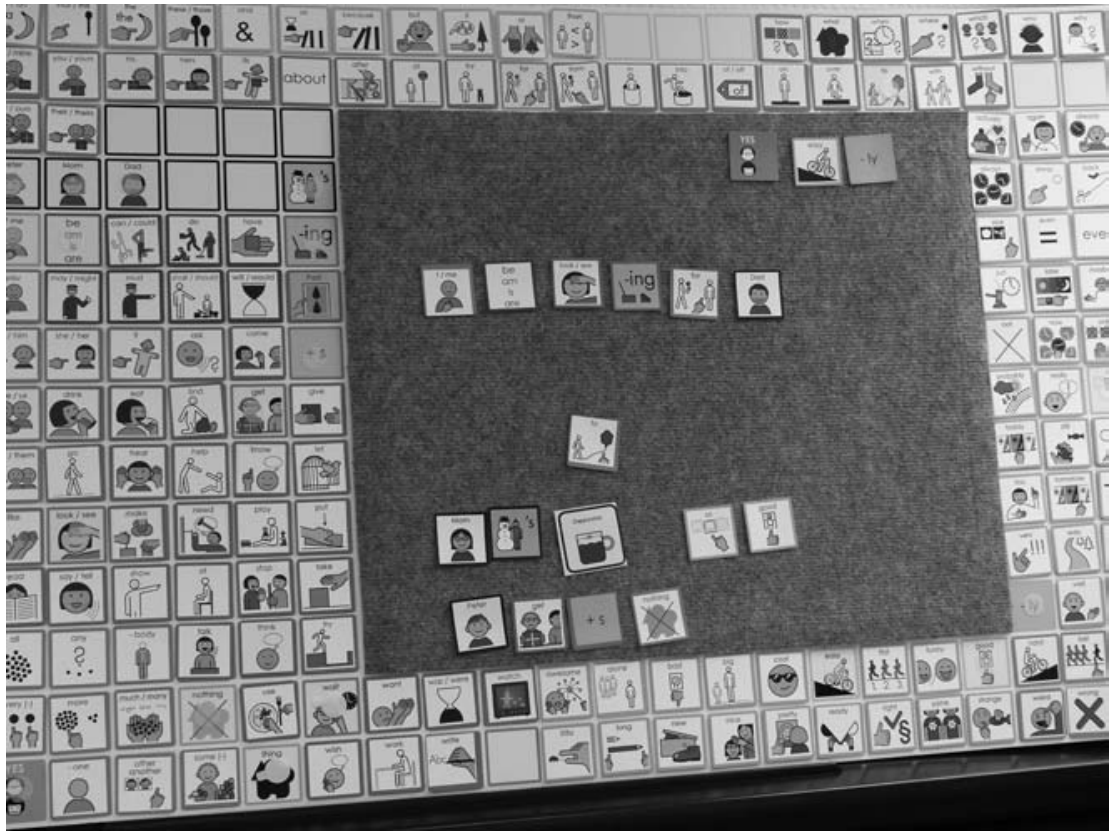


Abb. 7: Kernvokabularbasierter englischer Kommunikationsordner (Symbole () METACOM)

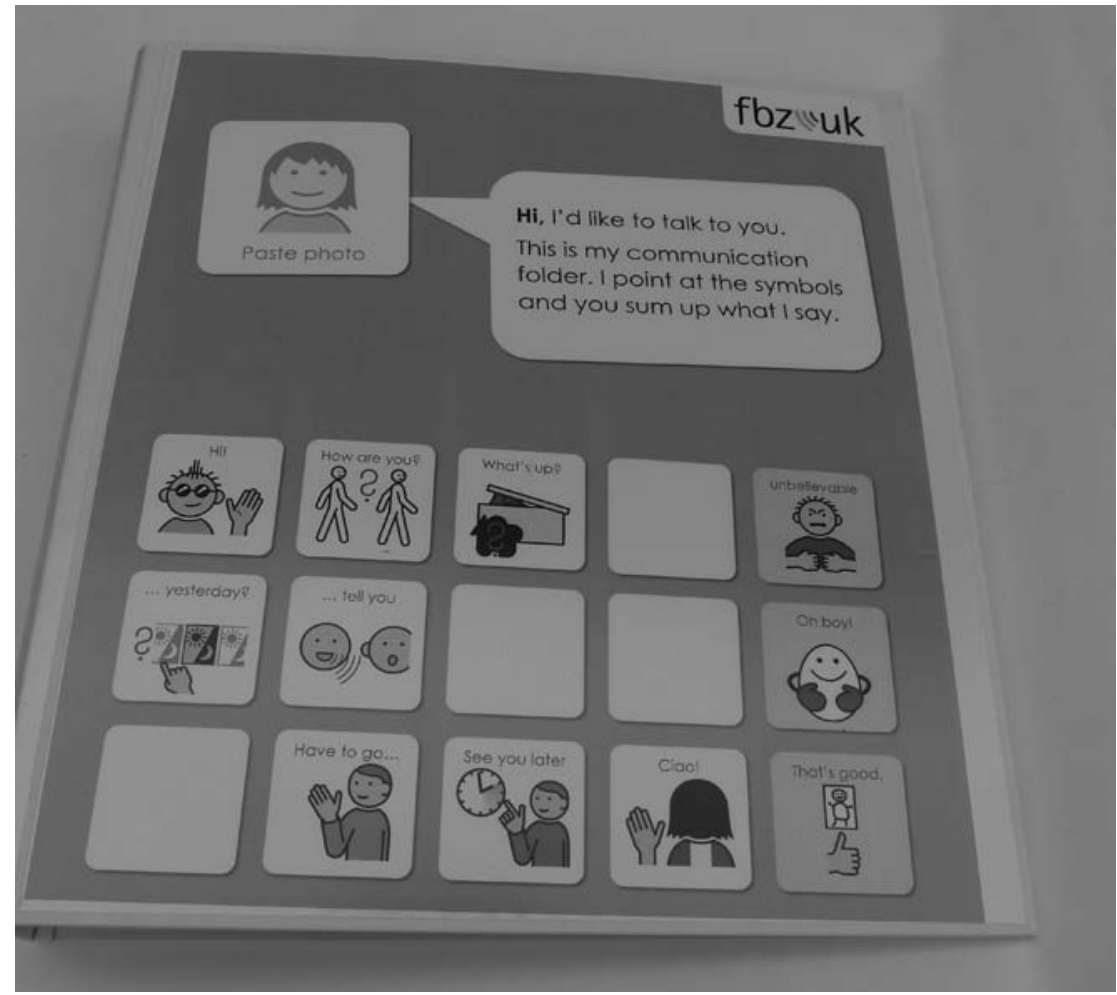


Abb. 8: Elektronische Kommunikationshilfe MyCore (deutschlenglisch)

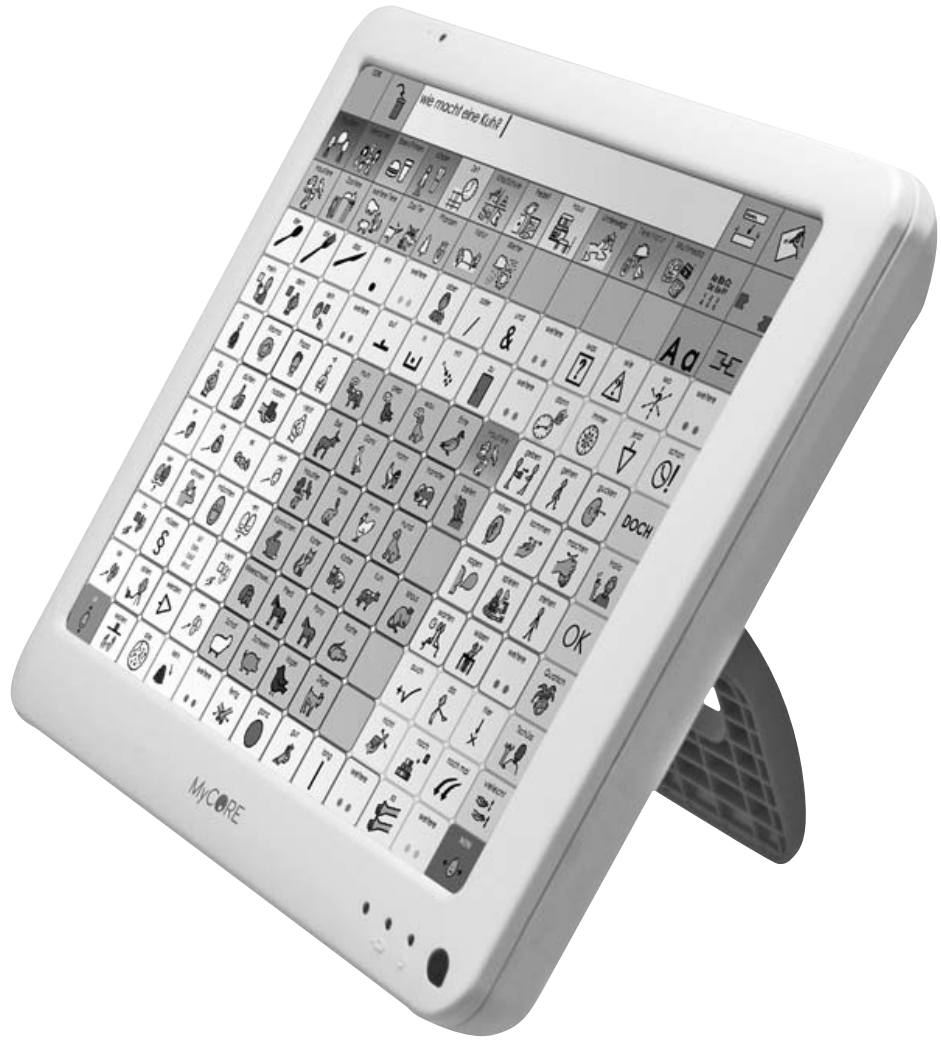

Unterstützt kommunizierende Kinder, die die Kölner Kommunikationsmaterialien (Deutsch) für ihre Alltagskommunikation nutzen, haben trotz möglicher Lernerschwernisse und Sprachentwicklungsbeeinträchtigungen den Vorteil, dass die Anordnung der Wortarten im deutschen und englischen Kommunikationssystem weitgehend identisch ist und sie somit - selbst bei fehlenden Schriftsprachkenntnissen - schnell und flexibel in beiden Sprachen kommunizieren können. Sie sind so Teil der Klassengemeinschaft. Die komplexe Kommunikationsstörung stellt keinen Grund des Ausschlusses mehr dar. Die Kinder können sich in Alltagssituationen weitgehend verständlich machen, da sie auch bei eingeschränktem Wortschatz und 
erschwertem Fremdspracherwerb einen direkten Zugriff auf 80\% der Alltagssprache haben.

Die Fokussierung auf das frühe Lernen von Kernvokabular im Fremdsprachunterricht ermöglicht allen Schülern einen schnellen Einstieg in die neue Sprache. Unterstützt durch Spiele, Lieder, Reime, Sport und andere Handlungen des Schulalltages erfahren alle Kinder die Kraft der Sprache bereits zu einem sehr frühen Zeitpunkt des Sprachenlernens (Klasse 1-2). Sprachbegegnungsunterricht kann so lebendig erfahren und umgesetzt werden, ohne dass den Kindern mit dem ungeliebten Vokabeltraining von im Alltag selten vorkommenden Inhaltswörtern die Freude und Motivation an der englischen Sprache bereits in der Frühphase des Fremdsprachlernens genommen wird. Die abschließenden Beispiele sollen exemplarisch einen Einblick in die Kraft und die Flexibilität des Kernvokabulars geben, wenn man sie didaktisch aufbereitet in den Englisch-Anfangsunterricht integriert. Darüber hinaus zeigen bereits diese wenigen Beispiele, dass mit einem kernvokabularbasierten Sprachförderunterricht immer auch das Lernen von Grammatik einhergeht.

\begin{tabular}{|c|c|c|}
\hline Spielidee & Schüler & Partner \\
\hline \multirow[t]{2}{*}{\begin{tabular}{|l|} 
Wer bin ich? (der \\
Spielpartner hat den \\
Namen oder das \\
Bild einer bekannten \\
Persönlichkeit auf \\
dem Rücken kleben)
\end{tabular}} & $\begin{array}{l}\text { Are you big? } \\
\text { Are you small? } \\
\text { Do you like singing? } \\
\text { Is singing your daily } \\
\text { work? }\end{array}$ & $\begin{array}{l}\text { No, I'm not big. } \\
\text { Yes, more or less I'm small. } \\
\text { Yes, I like singing. } \\
\text { Yes, more or less I get my } \\
\text { money with singing. }\end{array}$ \\
\hline & $\begin{array}{l}\text { Do I know you from the } \\
\text { TV? } \\
\text {... }\end{array}$ & $\begin{array}{l}\text { Yes, you could know me from } \\
\text { the TV. } \\
\ldots\end{array}$ \\
\hline $\begin{array}{l}\text { Gesichter-Memory } \\
\text { ( } 2-4 \text { Schüler } \\
\text { spielen Memory } \\
\text { mit bekannten } \\
\text { Gesichtern aus } \\
\text { Klasse, Schule, TV) }\end{array}$ & $\begin{array}{l}\text { Oh, I know him. That } \\
\text { is...? I cannot remember. } \\
\text { Who is he? } \\
\text { Anyway, I take another } \\
\text { card. } \\
\text { My turn. Stop. It's my } \\
\text { turn! I try this card and } \\
\text { this... oh, bad luck. } \\
\text { You are again! } \\
\text { And now I take this and } \\
\text { that. - What a pity, but } \\
\text { I know where the right } \\
\text { card is. It's your turn now. } \\
\text {... }\end{array}$ & $\begin{array}{l}\text { I don't know. I have never seen } \\
\text { him before. } \\
\text { Wrong. It's my turn. } \\
\text { Oh, yeah. I know the second } \\
\text { card... Wrong. What a pity. } \\
\text { My turn. I take this and that. } \\
\text { Yes, I get it. My first pair. } \\
\text { And now I take this and that } \\
\text { Oh, bad luck. Maybe next time }\end{array}$ \\
\hline
\end{tabular}




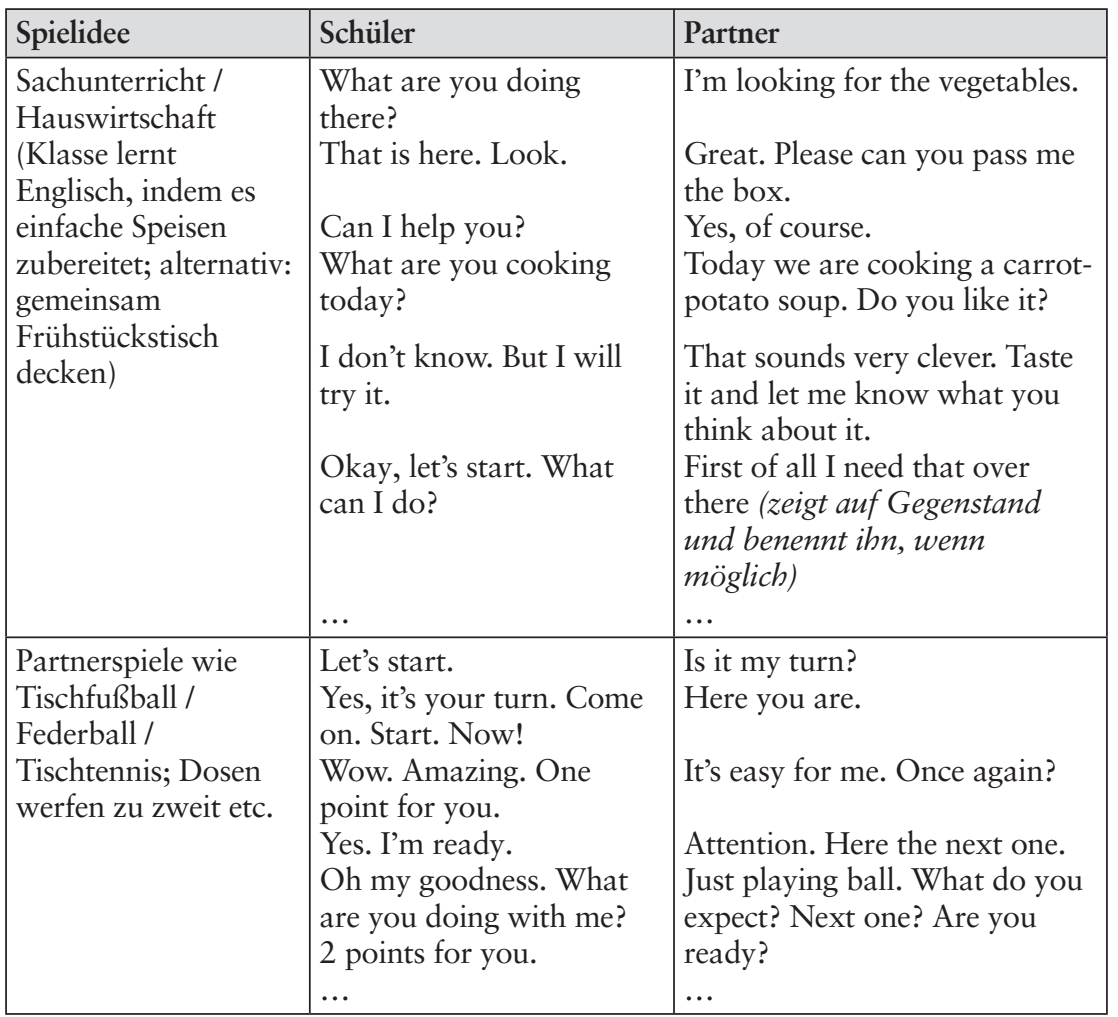

Für den Anfangsunterricht bietet sich ein Verhältnis von 70\% Kernvokabular und 30\% Randvokabular (überwiegend Inhaltswörter) an, um das universelle Kernvokabular zu lernen, ohne auf spezifische Inhalte und Themen in der Vermittlung ganz verzichten zu müssen (vgl. Sachse \& Willke 2011). Erst nach dem sicheren Erwerb des Kernvokabulars erfolgt eine didaktische Verschiebung auf Inhaltswörter, um den Wortschatz sukzessive zu erweitern. Der Fokus auf Kernvokabular in der Frühphase des Fremdsprachlernens ermöglicht eine auf Alltagssprache und die pragmatische Kompetenz ausgerichtete Sprachförderung, die Kindern mit Lernschwierigkeiten einen erleichterten Zugang zur Fremdsprache ermöglicht und die selbst Kinder mit schweren Behinderungen nicht ausschließen muss, weil ihr didaktischer Ansatz bereits inklusiv ist. 


\section{Literaturverzeichnis}

Aktas, Maren (Hrsg.) (2012), Entwicklungsorientierte Sprachdiagnostik und -förderung bei Kindern mit geistiger Behinderung. Theorie und Praxis. München: Elsevier, Urban \& Fischer.

Banajee, Meher; Dicarlo, Cynthia \& Stricklin, Sarintha Buras (2003), Core Vocabulary Determination for Toddlers. Augmentative and Alternative Communication 19: 2, 67-73.

Bergeest, Harry; Boenisch, Jens \& Daut, Volker (2015), Körperbehindertenpädagogik. Grundlagen, Förderung, Inklusion (5. Aufl.). Bad Heilbrunn: Klinkhardt.

Bernasconi, Tobias \& Böing, Ursula (2015), Pädagogik bei schwerer und mehrfacher Behinderung. Stuttgart, Oberhausen: Kohlhammer, im Druck.

Beukelman, David; Jones, Rebecca \& Rowan, Mary (1989), Frequency of word usage by nondisabled peers in integrated preschool classrooms. Augmentative and Alternative Communication 5: 4, 243-248.

Boenisch, Jens (2003), Zur Situation unterstützt kommunizierender Kinder und Jugendlicher an Schulen für Körperbehinderte und Geistigbehinderte in Deutschland. In: Boenisch, Jens \& Bünk, Christof (Hrsg.) (2003), Methoden der Unterstützten Kommunikation. Karlsruhe: Von Loeper Literaturverlag, 19-35.

Boenisch, Jens (2009), Kinder ohne Lautsprache. Grundlagen, Entwicklungen und Forschungsergebnisse zur Unterstützten Kommunikation. Karlsruhe: Von Loeper Literaturverlag.

Boenisch, Jens (2014), Kernvokabular im Kindes- und Jugendalter: Vergleichsstudie zum Sprachgebrauch von Schülerinnen und Schülern mit und ohne geistige Behinderung und Konsequenzen für die UK. $u k$ \& forschung, 4-23; Sonderbeilage Unterstützte Kommunikation 1/2014.

Boenisch, Jens \& Sachse, Stefanie Kalén (2007), Sprachförderung von Anfang an. Unterstützte Kommunikation 3, 12-20.

Boenisch, Jens \& Soto, Gloria (2015), The Oral Core Vocabulary of Typically Developing English-Speaking School-Aged Children: Implications for AAC Practice. Augmentative and Alternative Communication 31: 1, 77-84.

Braun, Ursula (2008), Was ist Unterstützte Kommunikation. In: von Loeper \& ISAAC (Hrsg.) (2008), Handbuch der Unterstützten Kommunikation. Karlsruhe: Von Loeper, 0.003.01-01.005.01. 
BRK (Behindertenrechtskonvention) (2009), UN-Behindertenrechtskonvention. [Online: www.behindertenrechtskonvention.info/. 16.03.2015].

Clendon, Sally A. \& Erickson, Karen A. (2008), The Vocabulary of Beginning Writers: Implications for Children with Complex Communication Needs. Augmentative and Alternative Communication 24: 4, 281-293.

Grimm, Hannelore \& Weinert, Sabine (2002), Sprachentwicklung. In: Oerter, Rolf \& Montada, Leo (Hrsg.) (2002), Entwicklungspsychologie (5. Aufl.). Weinheim: Beltz, PVU, 517-550.

Robillard, Manon; Mayer-Crittenden, Chantal; Minor-Corriveau, Michèle \& Bélanger, Roxanne (2014), Monolingual and bilingual children with and without primary language impairment: Core vocabulary comparison. Augmentative and Alternative Communication 30: 3, 267-278.

Sachse, Stefanie Kalén \& Boenisch, Jens (2009), Kern- und Randvokabular in der Unterstützten Kommunikation: Grundlagen und Anwendung. In: von Loeper \& ISAAC (Hrsg.) (2009): Handbuch der Unterstützten Kommunikation. Karlsruhe: Von Loeper Literaturverlag, 01.026.3001.026.040.

Sachse Stefanie K.; Schmidt, Lena \& Wagter, Jacqueline (2013), Das Kölner Vokabular. In: Hallbauer, Angela; Hallbauer, Thomas \& Hüning-Meier, Monika (Hrsg.) (2013), UK kreativ. Wege der Unterstützten Kommunikation. Karlsruhe: Von Loeper Literaturverlag, 35-53.

Sachse, Stefanie K. \& Willke, Melanie (2011), Fokuswörter in der Unterstützten Kommunikation. In: Bollmeyer, Henrike; Engel, Kathrin; Hallbauer, Angela \& Hüning-Meier, Monika (Hrsg.) (2011): UK inklusive. Teilhabe durch Unterstützte Kommunikation. Karlsruhe: Von Loeper Literaturverlag, 375-394.

Trembath, David; Balandin, Susan \& Togher, Leanne (2007), Vocabulary selection for Australian children who use augmentative and alternative communication. Journal of Intellectual \& Developmental Disability 32: 4, 291-301. 


\section{Anhang}

Liste der 50 am häufigsten gesprochenen englischen Wörter von Schülern mit Englisch als Erstsprache (Native Speaker) und English as Second Language Schülern (ESL Schüler) Erstveröffentlichung mit Top 300 Wortliste siehe: Boenisch \& Soto (2015)

\begin{tabular}{|c|c|c|c|c|c|}
\hline \multicolumn{3}{|c|}{$\begin{array}{c}\text { Native Speaker } \\
\text { (Grades } 2-8 ; n=27 \\
\text { total words: } 98,053 \text { ) }\end{array}$} & \multicolumn{3}{|c|}{$\begin{array}{c}\text { ESL Schüler } \\
\text { (Grades } 2-8 ; n=10 ; \\
\text { total words: } 19,318 \text { ) }\end{array}$} \\
\hline Rank & Word & $\begin{array}{c}\text { Word } \\
\text { frequency }\end{array}$ & Rank & Word & $\begin{array}{c}\text { Word } \\
\text { frequency }\end{array}$ \\
\hline 1 & be & 7097 & 1 & be & 1322 \\
\hline 2 & I & 4261 & 2 & I & 1067 \\
\hline 3 & it & 3377 & 3 & it & 701 \\
\hline 4 & you & 3266 & 4 & you & 678 \\
\hline 5 & do & 2426 & 5 & do & 596 \\
\hline 6 & $a$ & 2200 & 6 & not & 421 \\
\hline 7 & not & 2099 & 7 & to & 419 \\
\hline 8 & to & 2093 & 8 & like & 383 \\
\hline 9 & the & 2080 & 9 & that & 340 \\
\hline 10 & that & 1663 & 10 & what & 329 \\
\hline 11 & no & 1339 & 11 & the & 328 \\
\hline 12 & and & 1311 & 12 & go & 317 \\
\hline 13 & go & 1286 & 13 & yeah & 297 \\
\hline 14 & have & 1231 & 14 & this & 292 \\
\hline 15 & what & 1173 & 15 & no & 281 \\
\hline 16 & like & 1158 & 16 & one & 253 \\
\hline 17 & oh & 1087 & 17 & oh & 228 \\
\hline 18 & this & 1077 & 18 & get & 221 \\
\hline 19 & my & 1002 & 19 & and & 220 \\
\hline 20 & can & 994 & 20 & my & 202 \\
\hline 21 & get & 962 & 21 & know & 194 \\
\hline 22 & we & 882 & 22 & me & 179 \\
\hline 23 & know & 827 & 23 & have & 172 \\
\hline 24 & me & 803 & 24 & we & 168 \\
\hline
\end{tabular}




\begin{tabular}{|c|c|c|c|c|c|}
\hline \multicolumn{3}{|c|}{$\begin{array}{c}\text { Native Speaker } \\
\text { (Grades } 2-8 ; n=27 \\
\text { total words: } 98,053 \text { ) }\end{array}$} & \multicolumn{3}{|c|}{$\begin{array}{c}\text { ESL Schüler } \\
\text { (Grades } 2-8 ; n=10 ; \\
\text { total words: } 19,318 \text { ) }\end{array}$} \\
\hline 25 & in & 761 & 25 & can & 167 \\
\hline 26 & yeah & 719 & 26 & on & 160 \\
\hline 27 & one & 706 & 27 & here & 145 \\
\hline 28 & just & 677 & 28 & in & 132 \\
\hline 28 & on & 677 & 29 & $\mathrm{a}$ & 130 \\
\hline 30 & so & 668 & 30 & he & 120 \\
\hline 31 & ok, okay & 658 & 31 & yes & 112 \\
\hline 32 & he & 645 & 32 & ok/okay & 103 \\
\hline 33 & of & 629 & 33 & say & 101 \\
\hline 34 & they & 576 & 34 & look & 97 \\
\hline 35 & yes & 549 & 35 & hey & 95 \\
\hline 36 & there & 539 & 35 & let & 95 \\
\hline 37 & say & 525 & 37 & she & 94 \\
\hline 38 & here & 461 & 38 & good & 89 \\
\hline 39 & will & 450 & 38 & there & 89 \\
\hline 40 & for & 441 & 40 & come & 82 \\
\hline 41 & look & 440 & 40 & right & 82 \\
\hline 42 & want & 430 & 42 & now & 81 \\
\hline 43 & your & 428 & 42 & put & 81 \\
\hline 44 & but & 425 & 44 & at & 79 \\
\hline 45 & because & 393 & 44 & make & 79 \\
\hline 46 & see & 391 & 44 & your & 79 \\
\hline 47 & at & 374 & 47 & so & 77 \\
\hline 48 & make & 344 & 48 & up & 74 \\
\hline 49 & how & 342 & 49 & how & 73 \\
\hline 50 & wait & 337 & 49 & with & 73 \\
\hline
\end{tabular}


Henning Rossa

\section{Lerngelegenheiten im inklusiven Englischunterricht für Schülerinnen und Schüler mit Förderbedarf im Bereich der geistigen Entwicklung}

Students with mental disabilities by and large have been excluded from EFL lessons in the mainstream classrooms of Germany's selective education system. The article discusses joint teaching opportunities for these and other learners with special needs in all types of educational contexts. It highlights pedagogical principles und concepts to be implemented in inclusive English language teaching to the benefit of all students.

\section{Einleitung: Inklusion als Entwicklungsaufgabe - für das Bildungssystem und die Fremdsprachendidaktik}

Seit Inkrafttreten der UN-Behindertenrechtskonvention im Jahr 2009 (vgl. United Nations 2006) hat sich in Deutschland unter dem Schlagwort Inklusion eine kontroverse Diskussion zur Umsetzung der in der Konvention konkretisierten universellen, uneingeschränkten Rechte auf Gleichstellung, Selbstbestimmung und gesellschaftliche Teilhabe von Menschen mit Behinderungen entwickelt. Dies gilt insbesondere für das in Artikel 24 festgeschriebene Recht, „nicht aufgrund von Behinderung vom allgemeinem Bildungssystem ausgeschlossen [zu] werden“ (Beauftragte der Bundesregierung für die Belange von behinderten Menschen 2014: 35).

Die öffentliche Diskussion kreist dabei im Kern um die Frage, inwiefern ein gemeinsames Lernen von Kindern mit und ohne Behinderung möglich und für alle Beteiligten förderlich sein kann. Dieser eher zweiflerische Grundton ist nicht verwunderlich, weil alle, die an der Diskussion teilnehmen, an ein Bildungssystem gewöhnt sind, das strukturell und - im wahrsten Sinne - selbstverständlich darauf ausgerichtet ist, Kinder und Jugendliche aufgrund individueller Merkmale zu sortieren, d.h. mit Blick auf unterschiedliche Bildungsangebote die Einen zu selektieren und die Anderen auszuschließen. 
Die unterzeichnenden Vertragsstaaten verpflichten sich in der UN-Konvention aber, ein „integratives Bildungssystem auf allen Ebenen “ ${ }^{1} \mathrm{zu}$ gewährleisten. In einem aktuellen Bericht für die Vereinten Nationen über die Entwicklungen, die in Deutschland im Sinne der Konvention angestoßen wurden, muss gegenwärtig festgestellt werden: „Von einem inklusiven Bildungssystem ist der Vertragsstaat weit entfernt “ (Monitoring-Stelle zur UN-Behindertenrechtskonvention 2015: 27).

Die skeptisch formulierte Frage, ob es in Deutschland überhaupt möglich sei, das in der Konvention festgeschriebene Menschenrecht auf Zugang zu einem inklusiven Schulsystem zu gewähren, ist vermutlich auch darauf zurückzuführen, dass zwar seit mehr als 40 Jahren $^{2}$ konzeptionelle und praktische Anstrengungen unternommen werden, das gemeinsame Lernen von Kindern mit und ohne Behinderungen zu fördern (vgl. Deutscher Bildungsrat 1973), diese aber im öffentlichen Diskurs kaum wahrgenommen wurden. In den meisten Fällen gingen diese Bemühungen nämlich von Eltern behinderter Kinder aus, die in der Gesellschaft und in der Bildungspolitik lange auf Skepsis und Ablehnung trafen (vgl. Roebke \& Hüwe 2006). In der schulischen Praxis sind dennoch eine Vielzahl von Projekten entwickelt worden, die gemeinsames Lernen im Elementarbereich, in der Grundschule und darüber hinaus erproben und erforschen (vgl. Eberwein 1988; Klauß \& Terfloth 2013; Link 2000; Ministerium für Schule und Weiterbildung des Landes Nordrhein-Westfalen (MSW NRW) 2012).

In der Diskussion um die Umsetzung der UN-Konvention im Bereich der schulischen Bildung ist andererseits zu beobachten, wie selbst Inklusionsbefürworter empfehlen, Kinder und Jugendliche mit geistigen und mehrfachen Behinderungen $^{3}$ unter Hinweis auf ihre besonders deutlichen und vielfältigen Einschränkungen bzw. von der Norm abweichenden Lernbedarfe absurderweise von der nun angestrebten Inklusion auszuschließen (vgl. Fischer \& Markowetz 2015; Fritz 2014). So sind in einer aktuellen Umfrage im

1 Dies ist die offizielle deutschsprachige Übersetzung; im englischen Original heißt es "an inclusive education system on all levels" (ibid.).

21975 wurde die erste Integrationsklasse in der Fläming-Grundschule in Berlin installiert.

3 Dies sind immerhin knapp 17 Prozent aller Schülerinnen und Schüler mit sonderpädagogischen Förderbedarf (vgl. KMK 2010: XI). 
Auftrag des Vereins Lebenshilfe nur 23 Prozent der Befragten (repräsentativ für die Bevölkerung in Deutschland über 16 Jahre) der Meinung, Kinder mit einer geistigen Behinderung könnten mit anderen Kindern zusammen an einer regulären Schule unterrichtet werden (vgl. Bundesvereinigung Lebenshilfe 2014: 14).

Unabhängig von einer Positionierung zu der Frage, unter welchen Bedingungen Lernende mit besonderem Förderbedarf im Bereich der geistigen Entwicklung optimal in ihrer Entwicklung und Bildung unterstützt werden können, ist offensichtlich, dass die hier skizzierte Haltung der intendierten Bedeutung des Begriffs Inklusion widerspricht. Inklusion verfolgt ein Ziel, das in der Erläuterung zum Begriff Behinderung im Anhang der von der Beauftragten der Bundesregierung für die Belange von behinderten Menschen herausgegebenen Publikation zur UN-Konvention in einfacher Sprache zusammengefasst wird: „Es soll keine Hindernisse geben. Dann wird kein Mensch behindert“ (2014: 77).

Das Recht auf Teilhabe an den Angeboten des allgemeinen Bildungssystems muss selbstverständlich auch den Zugang zum Erlernen einer Fremdsprache beinhalten. Dies gilt schon alleine deswegen, weil Unterricht in einer Fremdsprache eine Grundbedingung für jeden Schulabschluss ist. Für das Fach Englisch als dominante Schulfremdsprache ist es daher dringend angezeigt, dass sich die Fachdidaktik stärker als bislang mit der Frage auseinandersetzt, wie im Englischunterricht Bedingungen geschaffen werden können, die den Fähigkeiten und Bedürfnissen von Lernenden mit Behinderungen gerecht werden.

Die unterrichtsbezogenen Konzepte, die den fremdsprachendidaktischen Diskurs gegenwärtig bestimmen (z.B. Kommunikation, Interaktion, Interkulturalität, kulturelle Dezentrierung, Perspektivwechsel, Fokus auf Sprachbewusstheit), legen nahe, dass Inklusion im Sinne eines konstruktiven Umgangs mit Vielfalt und Individualität im Prinzip bereits zum Selbstverständnis des Faches gehöre. Ein Blick auf den Stand der konzeptionellen und empirisch orientierten fachdidaktischen Forschung zeigt jedoch, dass es sowohl an theoretischen Erklärungsansätzen als auch an konkretisierten, praxisorientierten Konzepten zur Anpassung der unterrichtlichen Lerngelegenheiten an die Bedürfnisse von Lernenden mit besonderem Förderbedarf mangelt (vgl. Schuppener, Bernhardt, Hauser \& Poppe 2014; Seitz 2004). 
Dieser blinde Fleck fachdidaktischer Forschung ist auf die historisch gewachsene Neigung zurückzuführen, den sogenannten „lernschwachen“ Schülerinnen und Schülern nur wenig Aufmerksamkeit zu schenken (vgl. Dörnyei 2005; Ganschow \& Sparks 2001; Rubin 1975; Trautmann 2010). Nach einer Hochphase in den 1960er und 70er Jahren im Kontext der Einführung von Englisch als Pflichtfach an Hauptschulen wurde zumindest im deutschsprachigen Kontext „die Weiterentwicklung einer Didaktik für leistungsschwächere Schüler vernachlässigt“ (Haß \& Kieweg 2012: 11; vgl. Gutschow 1978; Hellwig \& Sauer 1984).

Die Inklusion ist daher selbst im recht engen Sinne einer „anerkennenden Haltung “ (Seitz 2011) gegenüber verschiedenen Befähigungen zum und Behinderungen des Lernens im Englischunterricht noch weit davon entfernt, tatsächlich als zentrales fremdsprachendidaktisches Prinzip (vgl. Küchler \& Roters 2014) zu gelten.

Die These, die der hier vorliegende Beitrag anbietet, lautet: Eine inklusive Konzeption des Fremdsprachenunterrichts muss eine Didaktik der Vielfalt entwickeln, die verschiedene Bedürfnisse und Lernvoraussetzungen wertschätzt und bei der Auswahl angemessener Unterrichtsprinzipien, Inhalte und Lernformen berücksichtigt. Wenn wir Inklusion folgerichtig als Entwicklungsaufgabe begreifen, dann müssen wir aus pragmatischen Gründen anerkennen, dass die Didaktik des traditionellen Englischunterrichts in allgemeinbildenden Schulen zunächst mehr über geeignete Angebote für Lernende mit sonderpädagogischem Förderbedarf lernen muss. Auf dem Weg dahin mag es lohnend sein, etablierte Konzepte des kommunikativen und aufgabenorientierten Fremdsprachenunterrichts zu prüfen (vgl. Börner 2005) und mit Konzepten der Pädagogik und Didaktik in den Förderschwerpunkten zu verknüpfen (vgl. Pitsch \& Thümmel 2011).

\section{Fremdsprachliche Bildungsangebote für Schülerinnen und Schüler im Förderschwerpunkt Geistige Entwicklung}

Die Lernvoraussetzungen von Schülerinnen und Schülern im Förderschwerpunkt Geistige Entwicklung sind für den Erwerb einer Fremdsprache in der Regel als ungünstig einzuschätzen (vgl. Wember 2003). Dies gilt insbesondere bei diagnostizierten Entwicklungsstörungen, die mit der Reifung des Zentralnervensystems verknüpft sind (vgl. Deutscher Bildungsrat 1973: 37; 
WHO 2011). Wenn die allgemeinen kognitiven Funktionen, wie Wahrnehmung, Aufmerksamkeit, Gedächtnis, Wiedererkennen und Generalisieren, eingeschränkt sind, wenn die Sprachentwicklung selbst in der Muttersprache beeinträchtigt ist, wird Lernen, das vornehmlich auf sprachliche Vermittlung basiert, außerordentlich mühsam.

Es gibt zweifellos viele Lernbereiche, die für die praktische Lebensbewältigung und die Ausübung einer möglichst selbständigen gesellschaftlichen Teilhabe der Kinder, Jugendlichen und jungen Erwachsenen an der Förderschule Geistige Entwicklung wichtiger sind als Unterricht in einer Fremdsprache. ${ }^{4}$ Und dennoch wäre es arrogant und ignorant, diese in Bezug auf ihre Lernfähigkeiten und -schwierigkeiten besonders inhomogene Gruppe grundsätzlich vom Englischunterricht auszuschließen.

Printz (2014) hat im Rahmen einer Pilotstudie die Einstellungen zu und Erfahrungen mit Unterrichtsangeboten in der Fremdsprache Englisch von Lernenden und Lehrenden im Förderschwerpunkt Geistige Entwicklung untersucht und gewährt so Einblicke in Perspektiven der Beteiligten, die eine Legitimation fremdsprachlicher Bildungsangebote für diese Zielgruppe unterstützen können:

Also, nich' alle von meine Freunde lernen, haben Englisch gelernt. Aber auch wir Behinderte wollen das, was die anderen machen, auch dürfen. Das ist doch, ähm, Inklusion, nennt man das. So stell' ich mir dat vor. Das is' auch wichtig, damit man sich ma' im Urlaub und so unterhalten kann. Das können ja fast alle Leute. In Ausland, fänd', find ich gut, wenn ich mit andere, anderen Leuten mich unterhalten kann. Das wär super. (Verbale Daten aus Printz 2014, Interview mit S3)

Die Äußerungen eines Auszubildenden, der zuvor auf einer Schule mit dem Förderschwerpunkt Geistige Entwicklung an Unterrichtsangeboten in der Fremdsprache Englisch teilnehmen konnte, machen deutlich, dass eine pauschale Aberkennung der Relevanz fremdsprachlicher Unterrichtsangebote für die Bildungswege von Menschen mit geistiger Behinderung nicht $\mathrm{zu}$ halten ist: In fremdkulturellen Kontexten kann eine Fremdsprache allen

4 In den gesetzlichen Vorgaben zur Gestaltung des Unterrichts in der Förderschule Geistige Entwicklung kommt der Begriff Fremdsprache daher in der Regel nicht vor (vgl. z.B. Bayerisches Staatsministerium für Unterricht und Kultus 2003: 89 ff.; MSW NRW 2014: 5). 
Menschen die wertvolle Erfahrung ermöglichen, dass sie selbst mit rudimentären Kenntnissen in der Lage sind, ihren Handlungsspielraum über ihre bekannte Lebenswelt hinaus zu erweitern (vgl. Degen 1999: 2; Kutscher 2005: 254-255).

Neben dieser interkulturellen Dimension mag das Lernen (in) einer Fremdsprache selbst auf einer elementaren Ebene als Gelegenheit gelten, einen zusätzlichen Zugang zur Einübung kognitiver Prozesse zu schaffen, die auch für andere Lernbereiche von fundamentaler Bedeutung sind, z.B. durch aufmerksames Zuhören, Übungen zur Lautwahrnehmung, durch Kommunikation und Interaktion.

\section{Making it work: Orientierungen und Prinzipien für den inklusiven Englischunterricht}

Angesichts der eingeschränkten Fähigkeiten vieler Lernender mit einer geistigen Behinderung, eigenes Handeln zu planen und zu vollziehen, Aufgaben selbständig zu gliedern (vgl. KMK 1998: 4), sprachlich vermittelten Inhalten aufmerksam und konzentriert zu folgen (vgl. noticing hypothesis, Schmidt 1990) und sich bereits Gelerntes zu merken, muss das von Steudle und Doert \& Nold (in diesem Band) ausgewählte Leitprinzip des handlungsbzw. aufgabenorientierten Lernens (vgl. Börner 2005; Mühl 1979) vermutlich mit einem kleinschrittigen Vorgehen verknüpft werden, das es den Lernenden unter Rückgriff auf unterschiedliche Unterstützungsangebote (visuelle Medien, Realia, Feedback, das die korrekte bzw. angemessene sprachliche Form widerspiegelt) ermöglicht, auch kleine Lernerfolge bewusst wahrzunehmen.

Die didaktische Anforderung besteht darin, die Stützstrategien, das scaffolding, und die Einteilung des „Lernwegs“ (Fischer 1999) in zu bewältigende Lernschritte abwägend einzusetzen. Bei einer übertriebenen Reduktion der inhaltlichen und sprachlichen Komplexität gehen ansonsten zwangsläufig der „Sinn- und Sachbezug“ (Fischer 2004: 40) und die Bedeutsamkeit des kommunikativen Kontextes verloren, die für das Fremdsprachenlernen so grundlegend wichtig sind (vgl. Kutscher 2005: 261).

Lernende mit Förderbedarf im Bereich der geistigen Entwicklung nötigen dem inklusiven Englischunterricht durch ihre besonderen Lernvoraussetzungen eine konsequente Umsetzung zentraler Prinzipien ab, die im Grunde 
für einen modernen, kommunikativen Fremdsprachenunterricht charakteristisch sein müssten:

- das Primat der Mündlichkeit

- die Auswahl lebensweltlich relevanter, bedeutsamer Inhalte

- die Orientierung an pragmatischen Kompetenzen, die sich auf alltägliche Situationen der Sprachverwendung in sozialen Kontexten beziehen

- die Anbahnung von Lernprozessen, in denen die Lernenden alle verfügbaren sprachlichen Ressourcen einsetzen, um Bedeutungen auszuhandeln

- ausreichend Zeit schaffen für Phasen des Wiederholens und sinnvollen Übens

Wie können diese Konzepte helfen, den inklusiven Englischunterricht mit Blick auf Lernende mit einer geistigen Behinderung zu adaptieren? Auf der Ebene sprachlicher Lerngegenstände wird in einem an der mündlichen Kommunikation orientierten Unterricht verhindert, dass Lernende den Erwerb eines zusätzlichen Systems der Schriftsprache als frustrierend erleben. Der Zugang zu den Bedeutungen fremdsprachlicher Wörter und Wendungen geschieht anhand konkreter Gegenstände in lebensnahen Situationen und kann durch bereits erlernte sprachliche Kompetenzen und Strategien, z.B. in der Muttersprache und im Bereich der unterstützten Kommunikation (vgl. Boenisch, in diesem Band), unterstützt werden. So können Lerngelegenheiten entstehen, die auf die Beeinträchtigungen kognitiver Funktionen, das eingeschränkte Welt- und Erfahrungswissen und die zumeist an naheliegenden Bedürfnissen des täglichen Lebens orientierten Interessen der Lernenden ausgerichtet sind.

Bislang werden die oben genannten Prinzipien jedoch in der Alltagspraxis eines - zumindest an den weiterführenden allgemeinbildenden Schulen - akademisch geprägten, in weiten Teilen schriftsprachlich dominierten und an grammatischer Progression orientierten Englischunterrichts eher vernachlässigt (vgl. Helmke, Helmke, Schrader, Wagner, Klieme, Nold \& Schröder 2008). Es ist zu vermuten, dass diese Unterrichtspraxis im Grunde nur aufrecht erhalten werden kann, solange Lehrerinnen und Lehrer in ihrer didaktischen Analyse von kognitiv eher leistungsstarken Lernenden ausgehen, die diese Art des Unterrichts ertragen oder ihn sogar einfordern, weil er ihren individuellen Lernstilen entgegen kommt. 
Pyfers \& Timmis (2007) zeigen in ihrem Bericht aus einem europäischen Projekt zum Englischunterricht mit erwachsenen, geistig behinderten und lernbehinderten Teilnehmenden, wie die beteiligten Lehrkräfte die Inhalte und Materialien ihrer Lernangebote an die Fähigkeiten und Interessen der Zielgruppe anpassen:

Our target group may not be aware of what language is, or how people use language. They may have no or limited grammatical awareness (word order, sentence structure, etc.), even in their native language. Therefore, the English included in the curriculum, is 'ready to use English'. (Pyfers \& Timmis 2007: 10)

Diese inhaltliche Fokussierung auf chunks, d.h. Wortkombinationen, die die Lernenden in der Regel als Sinneinheit ganzheitlich verstehen und reproduzieren können, entspricht einer Umsetzung des in der Psycholinguistik, Zweitsprachenerwerbsforschung und Fremdsprachendidaktik etablierten Konzepts der Formelhaftigkeit von Sprache (vgl. Doughty \& Williams 1998; Meunier 2012; Schmitt 2004) unter behutsamer Berücksichtigung des förderpädagogischen Prinzips der kleinsten (Lern-) Schritte (vgl. Josef 1974; Pitsch \& Thümmel 2011: 188-189). Etwas nur scheinbar Triviales kommt noch hinzu: Die Strukturierung des Lernwegs in erfassbare Teile muss bei Lernenden mit einer geistigen Behinderung - im Vergleich zum herkömmlichen Fremdsprachenunterricht - mit deutlich mehr Gelegenheiten zum Wiederholen und Üben des Gelernten verknüpft werden:

Generally speaking, our target group does not learn by means of incidental learning. They will have to be confronted with words and phrases many times over, and in different communicative contexts. (Pyfers \& Timmis 2007: 17)

Der Blick auf Lernende mit einer geistigen Behinderung macht es außerdem erforderlich, die Illusion aufzugeben, man könne das Anforderungsniveau und die erreichbaren Lernziele für eine gesamte Lerngruppe auch nur annähernd verbindlich und präzise spezifizieren. Wenn Lernen trotz gravierend eingeschränkter kognitiver Funktionen gelingen soll, wird deutlich, dass der Lernerfolg besonders stark von der Frage abhängt, inwiefern der oder die Lernende einen persönlichen Zugang zum Lerninhalt wahrnehmen und verfolgen kann:

Many activities require the learners to use their prior knowledge of the world, to help them understand English words and phrases. The world referred to in the stories and activities resembles as much as possible the (often limited) world that our target group has personal experience with. (ibid.: 2) 
Die im traditionellen Unterricht bereits anerkannten Prinzipien der Lernerorientierung, Differenzierung und Individualisierung müssen demnach in einer inklusiv gedachten Englischdidaktik um das Prinzip der Personalisierung (vgl. OECD 2006; Weigand 2013) ergänzt werden. ${ }^{5}$ Personalisierung bedeutet in diesem Kontext, dass die Lernenden Gelegenheiten erhalten, die Inhalte und Formen des Lernens selbst zu bestimmen und sich in die Planung und Durchführung des Unterrichts mit ihren persönlichen Vorlieben und Interessen, Lernpotentialen, Talenten und Lernwegen einzubringen. Die wichtigste Herausforderung für den inklusiven Englischunterricht liegt dabei auf der Hand: Wie kann es angesichts der unumgänglichen Vielfalt inhaltlich, methodisch und sprachlich unterschiedlich strukturierter Lerngelegenheiten gelingen, dass Lehrende und Lernende ihre Auseinandersetzung mit der Fremdsprache tatsächlich als ein gemeinsames Projekt erleben?

\section{Zusammenfassung und Ausblick}

Dieser Beitrag analysiert und skizziert zentrale didaktische Konzepte des kommunikativen Fremdsprachenunterrichts und der Förderpädagogik, die im inklusiven Englischunterricht eingesetzt werden können, um fremdsprachliche Lerngelegenheiten zu schaffen, die den Interessen, Bedürfnissen und Fähigkeiten von Lernenden mit besonderem Förderbedarf im Bereich der geistigen Entwicklung gerecht werden.

Im Kern nimmt der Beitrag dabei die Perspektive einer Fremdsprachendidaktik ein, die sich bislang überwiegend mit der Modellierung universeller fremdsprachlicher Lern- und Entwicklungsprozesse und der konzeptionellen Weiterentwicklung des Unterrichts an allgemeinbildenden, weniger an berufsbildenden Schulen und kaum an Förderschulen beschäftigt hat. Aus dieser Perspektive, die bisher die Heterogenität der Lernvoraussetzungen nicht ausreichend in den Fokus gerückt hat, liegt es nahe, sich zunächst den Lernenden zu widmen, die noch mehrheitlich an Förderschulen mit dem Schwerpunkt Geistige Entwicklung unterrichtet werden. Aus dem Blick auf ihre Bedürfnisse, Fähigkeiten und Interessen ergibt sich die Frage, wie

5 Zerhusen (2012) zeigt anhand von Unterrichtsmaterialien (My book about me) für Lernende mit einer geistigen Behinderung in der Sekundarstufe 1, wie das Prinzip der Personalisierung im Englischunterricht konkret umgesetzt werden kann. 
durch individualisierte, kommunikative Lerngelegenheiten Barrieren aufgelöst werden können, die der Teilhabe von Kindern und Jugendlichen mit einer geistigen Behinderung am Englischunterricht im Weg stehen.

Gänzlich ungeklärt bleibt im Rahmen dieser grundlegenden Überlegungen die Frage, wie inklusiver Englischunterricht als gemeinsames Lernen organisiert und, angesichts der ausgeprägten Heterogenität der Lernvoraussetzungen und Lerngeschwindigkeiten von Lernenden mit und ohne besonderen Förderbedarf, gelingen kann. Es ist fraglich, ob die bislang vorliegenden Konzeptionen zur Differenzierung und individuellen Förderung im Fremdsprachenunterricht (vgl. Trautmann 2010) in der Praxis ausreichen, um angemessene Lernangebote für alle Schülerinnen und Schüler entwickeln zu können. Es wird wichtig sein, eine Balance aus ritualisierten und offeneren Unterrichtsformen, gemeinsamen und individuellen Lerngelegenheiten, Ruhe und Anregung zu schaffen und Freiräume für die Kontaktaufnahme und Kommunikation zwischen den Lernenden anzubieten (vgl. Klauß 2005: 304-307). Jetzt, da die schulische Inklusion Realität wird, ist es dringend notwendig, auf der Suche nach angemessenen Konzepten diejenigen Bildungsangebote zu untersuchen, in denen der gemeinsame Unterricht von Kindern und Jugendlichen mit unterschiedlichen Fähigkeiten und Interessen bereits gelingt.

\section{Author's note}

Ich schreibe diesen Beitrag einerseits aus der Perspektive eines Fremdsprachenforschers und -lehrers, der an der Sicht der handelnden Personen auf den Unterricht interessiert ist, und andererseits aus der Perspektive eines Vaters, der in den ersten sechs Lebensjahren seiner fröhlichen, empfindsamen, zugewandten und schwer geistig behinderten Tochter im Kontakt mit Bildungseinrichtungen des Elementarbereichs vielfältige Ausgrenzungserfahrungen gemacht hat. Ich bin immer wieder erstaunt über die keinen Widerspruch duldende Bestimmtheit, mit der andere Eltern und pädagogisch geschulte Personen im Bildungsbereich meine vorbehaltlose Zustimmung erwarten, wenn angeraten wird, man könne es anderen Kindern einfach nicht zumuten, mit meiner Tochter zusammen zu lernen, zu spielen oder Musik zu machen. Ich vermute, wie Georg Feuser (2014), dass Inklusion so lange nicht gelingen kann, wie „wir eine Einstellung haben, dass ein 
Mensch mit einer Beeinträchtigung, der mich vielleicht sogar ängstigt, weil ich nicht so sein will, wie der ist, ausgrenze, um seine Gegenwart nicht zu ertragen und damit stigmatisiere".

Ich wünsche meiner Tochter, dass sie in einer menschenfreundlicheren Gesellschaft, die Vielfalt nicht als Bedrohung empfindet, von Anderen angenommen wird; und zwar ungeachtet ihrer eingeschränkten feinmotorischen und koordinativen Fähigkeiten, ihrer idiosynkratischen Laut- und Körpersprache und ihres wenig entwickelten Verständnisses für Konventionen sozialer Interaktion.

In der Förderschule wird sie zurzeit intensiv dabei unterstützt, ihre lebenspraktischen Kompetenzen zu erweitern. Ihre Fähigkeiten und Interessen beziehen sich dabei auf Felder, die ganz eng mit ihrem direkten Erleben der Umwelt verknüpft und von den Inhalten und Zielen des herkömmlichen Fremdsprachenunterrichts scheinbar meilenweit entfernt sind. Und doch lacht sie gemeinsam mit ihrem Bruder über The Adventures of Abney and Teal im Kinderfernsehen der BBC, und ich kann nicht erkennen, wieso man ihr auf ihren schwer zu prognostizierenden Lernwegen die Gelegenheit verwehren sollte, an englischsprachigen Lernangeboten teilzuhaben.

\section{Literaturverzeichnis}

Bayerisches Staatsministerium für Unterricht und Kultus (2003), Lehrplan für den Förderschwerpunkt Geistige Entwicklung: Kommunikation und Sprache. [Online: https://www.isb.bayern.de/download/8918/kommunikation und sprache.pdf. 01.05.2015].

Beauftragte der Bundesregierung für die Belange von behinderten Menschen (2014), Die UN-Behindertenrechtskonvention. Übereinkommen über die Rechte von Menschen mit Behinderungen. [Online: https://www. behindertenbeauftragter.de/SharedDocs/Publikationen/DE/Broschuere UNKonvention_KK.pdf? blob=publicationFile. 01.05.2015].

Bundesvereinigung Lebenshilfe (2014), Gesellschaftliche Teilhabesituation von Menschen mit Behinderung. [Online: http://www.lebenshilfe.de/de/ presse/2014/artikel/Allensbach-Studie-Internet.pdf. 01.05.2015].

Boenisch, Jens (2015), Zur Bedeutung von Kernvokabular im inklusiven Englisch-Anfangsunterricht. In diesem Band.

Börner, Otfried (2005), Aufgabenorientiertes Fremdsprachenlernen - auch und gerade für Lerner mit sonderpädagogischem Förderbedarf. In: 
Müller-Hartmann, Andreas \& Schocker-von Ditfurth, Marita (Hrsg.) (2005), Aufgabenorientierung im Fremdsprachenunterricht: Task-based Language Learning and Teaching. Tübingen: Narr, 369-378.

Degen, Sabrina (1999), Integration im Englischunterricht. Chancen gemeinsamen Lernens für Kinder mit und ohne Behinderung. Berlin: Luchterhand.

Deutscher Bildungsrat (Hrsg.) (1973), Zur pädagogischen Förderung behinderter und von Behinderung bedrohter Kinder und Jugendlicher. Bonn: Bundesdruckerei.

Doert, Carolin \& Nold, Günter (2015), Integrativer Englischunterricht Forschungsfragen zwischen Wunsch und Wirklichkeit. In diesem Band.

Dörnyei, Zoltán (2005), The psychology of the language learner. Individual differences in second language acquisition. Mahwah, N.J. [u.a.]: Erlbaum.

Doughty, Catherine J. \& Williams, Jessica (1998), Pedagogical choices in focus on form. In: Doughty, Catherine J. \& Williams, Jessica (Hrsg.) (1998), Focus on Form in Classroom Second Language Acquisition. Cambridge: University Press, 197-285.

Eberwein, Hans (Hrsg.) (1988), Behinderte und Nichtbehinderte lernen gemeinsam. Handbuch der Integrationspädagogik. Weinheim und Basel: Beltz.

Feuser, Georg (2014), „Das Schulsystem macht Inklusion fast unmöglich“. Gespräch mit Korbinian Frenzel auf Deutschlandradio Kultur am 19.03.2014. [Online: http://www.deutschlandradiokultur.de/bildungschulsystem-macht-inklusion-fast-unmoeglich.1008.de.html?dram:article_ id $=280477$ 01.05.2015].

Fischer, Dieter (1999), Eine methodische Grundlegung. Würzburg: Edition Bentheim.

Fischer, Erhard (2004), Grundlagen und Prinzipien eines subjektorientierten Unterrichts im Förderschwerpunkt „geistige Entwicklung“. In: Fischer, Erhard (Hrsg.) (2004), Welt verstehen - Wirklichkeit konstruieren. Unterricht mit Kindern und Jugendlichen mit geistiger Behinderung. Dortmund: Borgmann, 13-51.

Fischer, Erhard \& Markowetz, Reinhard (Hrsg.) (2015), Inklusion im Förderschwerpunkt geistige Entwicklung. Stuttgart: Kohlhammer.

Fritz, Raphael (2014), Henri sollte für sein und das Wohl aller nicht auf das Gymnasium gehen. [Online: https://www.change.org/p/herr-stoch-henri- 
sollte-f\%C3\%BCr-sein-und-das-wohl-aller-nicht-auf-das-gymnasiumgehen. 01.05.2015].

Ganschow, Leonore \& Sparks, Richard (2001), Learning difficulties and foreign language learning: A review of research and instruction. Language Teaching 34: 2, 79-98.

Gutschow, Harald (1978), Eine Methodik des elementaren Englischunterrichts. Probleme und Arbeitsformen. Berlin: Cornelsen.

Haß, Frank \& Kieweg, Werner (2012), Englischunterricht für Schülerinnen und Schüler mit Lernschwierigkeiten. Seelze: Kallmeyer Klett.

Hellwig, Karlheinz \& Sauer, Helmut (Hrsg.) (1984), Englischunterricht für alle. Paderborn: Schöningh.

Helmke, Andreas; Helmke, Tuyet; Schrader, Friedrich-Wilhelm; Wagner, Wolfgang; Klieme, Eckhard; Nold, Günter \& Schröder, Konrad (2008), Alltagspraxis des Englischunterrichts. In: DESI-Konsortium (Hrsg.) (2008), Unterricht und Kompetenzerwerb in Deutsch und Englisch. Ergebnisse der DESI-Studie. Weinheim: Beltz, 371-381.

Josef, Konrad (1974), Lernen und Lernhilfen bei geistig Behinderten. Berlin: Marhold.

KMK (Kultusministerkonferenz) (1998), Empfehlungen zum Förderschwerpunkt geistige Entwicklung. [Online: http://www.kmk.org/fileadmin/pdf/ Presse UndAktuelles 2000/geist.pdf. 01.05.2015].

KMK (Kultusministerkonferenz) (2010), Sonderpädagogische Förderung in Schulen 1999 bis 2008. Statistische Veröffentlichungen der Kultusministerkonferenz. Dokumentation Nr. 189 - März 2010. [Online: http:// www.kmk.org/fileadmin/pdf/Statistik/Dokumentationen/Dok 189 SoPaeFoe 2008.pdf. 01.05.2015].

Klauß, Theo (2005), Ein besonderes Leben. Grundlagen der Pädagogik für Menschen mit geistiger Behinderung. Heidelberg: Winter.

Klauß, Theo \& Terfloth, Karin (Hrsg.) (2013), Besser gemeinsam lernen! Inklusive Schulentwicklung. Heidelberg: Universitätsverlag Winter.

Küchler, Uwe \& Roters, Bianca (2014), Embracing Everyone: Inklusiver Fremdsprachenunterricht. In: Amrhein, Bettina \& Dziak-Mahler, Myrle (Hrsg.) (2013), Fachdidaktik inklusiv - Auf der Suche nach didaktischen Leitlinien für den Umgang mit Vielfalt in der Schule. Münster: Waxmann, 233-248. 
Kutscher, Sandra (2005), Fremdsprachenlernen in der Sonderschule. Forschungsstand und Konsequenzen für den Englischunterricht bei Schülern mit Lernschwierigkeiten. Sonderpädagogische Förderung 50: 3, 253-269.

Link, Erika (2000), Eine Schule für alle? Schulische Integration in NordrheinWestfalen im historischen, pädagogischen, bildungspolitischen und juristischen Kontext. Frankfurt am Main [u.a.]: Lang.

Meunier, Fanny (2012), Formulaic Language and Language Teaching. Annual Review of Applied Linguistics 32, 111-129.

Ministerium für Schule und Weiterbildung des Landes Nordrhein-Westfalen (MSW NRW) (2012), PRIMUS: Schulversuch zur Erprobung des Zusammenschlusses von Schulen der PRIMarstufe Und der Sekundarstufe. [Online: https://www.schulministerium.nrw.de/docs/Schulsystem/Versuche/ Primus/Kontext/Eckpunkte Schulversuch PRIMUS.pdf. 01.05.2015].

Ministerium für Schule und Weiterbildung des Landes Nordrhein-Westfalen (MSW NRW) (2014), Verordnung über die sonderpädagogische Förderung, den Hausunterricht und die Schule für Kranke (Ausbildungsordnung sonderpädagogische Förderung - AO-SF). Frechen: Ritterbach.

Monitoring-Stelle zur UN-Behindertenrechtskonvention (2015), Parallelbericht an den UN-Fachausschuss für die Rechte von Menschen mit Behinderungen anlässlich der Prüfung des ersten Staatenberichts Deutschlands gemäß Artikel 35 der UN-Behindertenrechtskonvention. [Online: http:// www.institut-fuer-menschenrechte.de/fileadmin/user_upload/PDF-Dateien/Parallelberichte/Parallelbericht_an_den_UN-Eachausschuss_fuer_die Rechte von Menschen mit Behinderungen 150311.pdf. 01.05.2015].

Mühl, Heinz (1979), Handlungsbezogener Unterricht mit Geistigbehinderten. Bonn-Bad Godesberg: Dürr.

OECD (2006), Personalising Education, Schooling for Tomorrow. Paris: OECD Publishing.

Pitsch, Hans-Jürgen \& Thümmel, Ingeborg (2011), Zur Didaktik und Methodik des Unterrichts mit Geistigbehinderten. Oberhausen: Athena.

Printz, Sarah (2014), Potential Benefits of Teaching English as a Foreign Language to Learners with Mental Disabilities. Unveröffentlichte Masterarbeit, Institut für Anglistik und Amerikanistik, Fakultät Kulturwissenschaften, Technische Universität Dortmund. 
Pyfers, Liesbeth \& Timmis, Ivor (2007), English without Frontiers. Teaching English as a Foreign Language to Adult Learners with Intellectual Disabilities or Learning Difficulties. Methods, Activities, Materials. Hoensbroek, NL: Pragma Equal Access.

Roebke, Christa \& Hüwe, Birgit (2006), Elternbewegung gegen Aussonderung von Kindern mit Behinderungen: Motive, Weg und Ergebnisse. Zeitschrift für Inklusion 1: 1 [Online: http://www.inklusion-online.net/ index.php/inklusion-online/article/view/181/181 01.05.2015].

Rubin, Joan (1975), What the good language learner can teach us. TESOL Quarterly 9: 1, 41-51.

Seitz, Simone (2004), Forschungslücke inklusive Fachdidaktik - ein Problemaufriss. In: Sander, Alfred \& Schnell, Irmtraud (Hrsg.) (2004), Inklusive Pädagogik. Bad Heilbrunn: Klinkhardt, 215-231.

Seitz, Simone (2011), Eigentlich nichts Besonderes - Lehrkräfte für die inklusive Schule ausbilden. Zeitschrift für Inklusion 6: 3 [Online: http://www.inklusion-online.net/index.php/inklusion-online/article/ view/83/83. 01.05.2015].

Schmidt, Richard (1990), The role of consciousness in second language learning. Applied Linguistics 11, 129-158.

Schmitt, Norbert (Hrsg.) (2004), Formulaic Sequences. Acquisition, Processing and Use. Amsterdam: John Benjamins.

Schuppener, Saskia; Bernhardt, Nora; Hauser, Mandy \& Poppe, Frederik (Hrsg.) (2014), Inklusion und Chancengleichheit. Bad Heilbrunn: Klinkhardt.

Steudle, Ines (2015), Auf dem Weg zu einer inklusiven Englischdidaktik - Erkenntnisse zu Potentialen und Herausforderungen aus dem (Englisch-)Lernen von Schülerinnen und Schülern mit Förderbedarf im Bereich der geistigen Entwicklung. In diesem Band.

Trautmann, Matthias (2010), Heterogenität - (k)ein Thema der Fremdsprachendidaktik? Erweiterte Fassung eines Vortrags für die 47. Tagung der BAG Englisch, 2009. [Online: http://www.bag-englisch.de/wp-content/ uploads/2010/01/Heterogenität-Trautmann.pdf. 01.05.2015].

United Nations (2006), Convention on the Rights of Persons with Disabilities. [Online: http://www.un.org/disabilities/convention/conventionfull. shtml. 01.05.2015]. 
Weigand, Gabriele (2013), Person und Schulentwicklung. In: Krautz, Jochen \& Schieren, Jost (Hrsg.) (2013), Persönlichkeit und Beziehung als Grundlage der Pädagogik. Beiträge zur Pädagogik der Person. Weinheim: Juventa, 128-142.

Wember, Franz (2003), Englisch im Primarbereich an Sonderschulen. Chance oder Überforderung? [Online: http://www.schulentwicklung. nrw.de/cms/upload/egs/f wember chance o ueberforderungberforderung pdf 01.05.2015].

World Health Organziation (WHO) (2011), ICD-10. International Statistical Classification of Diseases and Related Health Problems. $10^{\text {th }}$ Revision. Genf: WHO Press.

Zerhusen, Renate (2012), Anfangsunterricht Englisch. My Book About Me: Unterrichtsideen und Materialien für Schüler mit geistiger Behinderung (5. bis 9. Klasse). Hamburg: AAP Lehrerfachverlage (Persen). 
Ines Steudle

\section{Auf dem Weg zu einer inklusiven Englischdidaktik - Erkenntnisse zu Potentialen und Herausforderungen aus dem (Englisch-) Lernen von Schülerinnen und Schülern mit Förderbedarf im Bereich der geistigen Entwicklung}

Inclusive teaching aims to provide opportunities for learning for everyone. The aim of the article is the identification of tangible reference points for inclusive English language teaching by showing the intersection of special needs education and EFL teaching methodology. The article hence focuses on students with special cognitive needs and derives methodological principles that can motivate inclusive (foreign language) teaching philosophy and strategies. The paper also discusses the challenges that exist on different levels and outlines first pedagogical recommendations for the successful learning of foreign languages. Key concepts of the article are task-based language learning and other hands-on teaching recommendations, and the importance of EFL instruction in identity formation and participation.

\section{Zum Verständnis von Inklusion}

Als Ziel von Inklusion kann eine zu erreichende Gleichwertigkeit der Individuen, Vielfalt in der Differenz sowie Partizipation, Anerkennung und Gerechtigkeit angesehen werden (vgl. Ziemen 2012: 3). Dementsprechend dürfen vorhandene individuelle Ressourcen geistiger, körperlicher oder emotionaler Art keine Selektionskriterien bilden, sondern vielmehr als Ausgangspunkt für individualisierende Maßnahmen einer inklusiven Pädagogik nutzbar gemacht werden. Spätestens seit der Ratifizierung der UN-Behindertenrechtskonvention durch die Bundesrepublik im Jahr 2009 befindet sich das deutsche Bildungssystem zunehmend auf der Suche nach einer alle Lerner umfassenden und gleichzeitig differenzierenden Didaktik. 


\section{Förderschule geistige Entwicklung - eine „Schule für Alle“}

Geistigbehinderte, Menschen mit geistiger Behinderung, Schülerinnen und Schüler mit Förderbedarf im Bereich geistige Entwicklung, Personen mit Lernschwierigkeiten - unabhängig von Bezeichnungen mag Einzelnen ein kulturorientiertes, formales Bildungsangebot wie der Erwerb der Zweitsprache Englisch für diesen Personenkreis aufgrund von Vorstellungen zu kognitiven Voraussetzungen, mangelnder Lebensbedeutsamkeit oder sozialem Status in der Gesellschaft auf den ersten Blick fragwürdig erscheinen. Hier ist ein Umdenken erforderlich: eine Pädagogik, die für sich beansprucht, inklusiv zu sein, schließt alle Lerner ein. Eine kategorische Unterscheidung in „inklusions-fähige“ und „inklusions-unfähige“ Personen ist nicht haltbar. Inklusive Didaktik will jedem Lerner alle Inhalte anbieten. Was offen bleibt, ist die Frage nach konkreten Wegen der Umsetzung. Wie können Grundsätze der Pädagogik für Menschen mit Förderbedarf im Bereich geistige Entwicklung Anregungen für die Entwicklung einer inklusiven Englischdidaktik liefern, was haben beide Disziplinen gemeinsam und wie können vorhandene Ressourcen im Sinne bewährter didaktischer Prinzipien als Schritte in diese Richtung nutzbar gemacht werden?

Für eine angestrebte inklusive Didaktik des Unterrichtsfaches Englisch ist es bedeutsam, bei einem System anzusetzen, das keine Mindestanforderungen für Teilhabe und Teilnahme stellt, also nicht selektiv vorgeht. Außerdem verlangt Inklusion nach Unterrichtsmodellen, welche Differenzierung und Individualisierung als selbstverständliche Kennzeichen für sich beanspruchen. Die genannten Kriterien sind dem Förderschwerpunkt geistige Entwicklung immanent. Zudem vereint diese Disziplin in ihrer Schülerschaft (ähnlich wie in anderen Förderschwerpunkten) ein derart großes Maß an Heterogenität, dass es sich hierbei bereits um ein mögliches Exemplar der vielfach angestrebten „Schule für Alle“ handelt. Neben denjenigen, die das System Förderschule geistige Entwicklung von Einschulung bis zur Berufsschule besuchen, ist die Zahl der Quereinsteiger aus anderen Schulformen, auch denen des allgemeinen Systems, wachsend. Um die in Bezug auf ihre kognitiven Voraussetzungen sowie soziale und kulturelle Herkunft ausgesprochen heterogenen Schülerinnen und Schüler zu fördern, muss die Zielvorstellung des Unterrichts dieser besonderen „Schule für Alle“ durchaus breit formuliert sein. Globales Leitziel des Förderschwerpunkt geistige 
Entwicklung stellt laut Lehrplan und KMK die Selbstverwirklichung jedes Einzelnen in sozialer Integration dar (vgl. Straßmeier 2000: 22). Zur Erreichung dieser ist der Schlüssel in einer individuellen Förderung zu sehen, die von der subjektiven Lebenswelt der Schülerschaft ausgeht: Unterricht muss den persönlichen Voraussetzungen des Individuums entsprechen und gleichzeitig soweit wie möglich dazu beitragen, die gesellschaftlichen Erwartungen zu erfüllen. Umgesetzt wird dies in Form von individuellen Förderplänen, die konkrete Ziele und Maßnahmen enthalten. Selbstverwirklichung in sozialer Integration - die Konsequenz daraus kann zu einer Argumentation für das Englischlernen von Menschen mit (geistiger) Behinderung herangezogen werden, beispielsweise weil es dem Wunsch und damit der Selbstverwirklichung einer Person entspricht, Englisch zu lernen oder weil Fremdsprachkompetenz als eine maßgebliche Bedingung anzusehen ist, den Anforderungen der Umwelt zu entsprechen (vgl. Steudle 2013: 3). Gleichzeitig wird eine solche Diskussion im inklusiven Setting hinfällig, da es keine Unterscheidung nach „Inklusionsfähigkeit“ bzw. geeigneten und ungeeigneten Inhalten geben darf.

\section{Handlungsorientierung als Weg zu inklusiver Englischdidaktik}

Handlungsorientierung ist kein neues methodisch-didaktisches Konzept, sondern hat seine Wurzeln bereits in reformpädagogischen Bewegungen Ende des 19. Jahrhunderts. Für den Förderschwerpunkt Geistige Entwicklung, allgemeiner: für den Unterricht für Schülerinnen und Schüler mit geistiger Behinderung wurde das Prinzip seit 1979 vor allem durch Mühl entwickelt. Dieser definierte Handeln als motiviertes, zielgerichtetes, geplantes und kontrolliertes Verhalten und baute darauf die Leitidee der Handlungsfähigkeit auf (vgl. Mühl 1991: 109). Im Handlungsorientierten Unterricht sollen „eigene Interessen und Bedürfnisse wahrgenommen und mitgeteilt werden und in möglichst realen, sozialen Situationen eingeschätzt werden “ (vgl. ebd.). Lernen soll dabei in ganzheitlichen Lebensvollzügen (kognitiv, pragmatisch, affektiv) stattfinden, wodurch Handlungsorientierter Unterricht zu mehr Mündigkeit der Lerner führen, ihr Interesse und ihre Selbsttätigkeit fördern und zu Selbstbestimmung und Individualisierung beitragen kann (vgl. ebd.). Die Bedeutsamkeit von Handlungsorientierung als Unterrichtsprinzip im Förderschwerpunkt geistige Entwicklung wird 
auch in aktuellerer Fachliteratur belegt, wonach grundsätzlich die Persönlichkeit und die individuellen Handlungskompetenzen der Schülerinnen und Schüler im Vordergrund von Unterrichtsbemühungen stehen und der Unterricht von Handlungsoffenheit bestimmt werden sollen (Pitsch \& Thümmel 2005: 22).

Auch in der Englischdidaktik sind Forderungen nach handelnder Interaktion (Bleyhl 2000) oder sprachlicher Handlungsfähigkeit (Bach \& Timm 2013) als Weg und Ziel des Fremdsprachunterrichts verbreitet. Der Ansatz der Handlungsorientierung findet sich auch in den Vorgaben des Gemeinsamen Europäischen Referenzrahmens CEFR, der Englischlerner als sozial Handelnde betrachtet, die kommunikative Aufgaben bewältigen und damit dem langfristigen Anspruch an fremdsprachliche Kompetenz ICC (Intercultural Communicative Competence) näher kommen: Englischunterricht soll die Schülerinnen und Schüler befähigen, zu aktiven handelnden, interkulturell kompetenten Individuen zu werden (vgl. Trim 2009: 21).

Als Konsequenz aus den bisherigen Ausführungen geht hervor, dass Unterrichtende (Er-) Kenntnisse der jeweiligen Disziplinen nutzen und auf die heterogene Schülerschaft in inklusiven Settings übertragen müssen. Inklusion erfordert einen (Englisch-) Unterricht, in dem die Lernenden selbst handelnd tätig werden.

\section{Potentiale in der Didaktik des Förderschwerpunkts geistige Entwicklung und Nutzungsmöglichkeiten für den Englischunterricht}

Wie deutlich wurde, beinhalten der Förderschwerpunkt geistige Entwicklung und die Englischdidaktik mehr Gemeinsamkeiten als bislang angenommen: beide wollen Lerner aktivieren und zum unterrichtlichen Handeln befähigen. Um das zu ermöglichen, sollte einer inklusiven Englischdidaktik ein konstruktivistisches Verständnis von Lernen zugrunde liegen, die eine Öffnung des Unterrichts in curricularer, inhaltlicher und methodischer Form soweit wie möglich zulässt, um auf diesem Wege der heterogenen Lernerschaft in inklusiven Settings zu begegnen. Hierbei sind insbesondere die Lernerorientierung bei der Wahl von Inhalten, die Gestaltung der Lernumgebung, der Einsatz entsprechender Methoden, die Rolle des Lehrenden 
sowie Prinzipien der konkreten Unterrichtsgestaltung zu berücksichtigen. Auf diese Aspekte soll auf Basis der Workshop-Diskussionen von Lehrerinnen und Lehrern aller Schulformen, Lehrende der Universität und des Zentrums für Lehrerbildung sowie Studierende des Faches Englisch verschiedener Schulformen im Rahmen der Fachtagung „Englisch Inklusiv“ der Universität Köln 2013 sowie vor dem Hintergrund der Literatur einschlägiger Fachdidaktik eingegangen werden.

Zunächst sollten Unterrichtende in inklusiven Settings bei der Planung handelnder Unterrichtsangebote im Fach Englisch grundsätzlich vom Lerner in seiner Individualität ausgehen, also sich eine Übersicht zu sozialer und kultureller Herkunft sowie Neigungen und weiteren Besonderheiten verschaffen. Dabei handelt es sich je nach Klassengröße und -zusammensetzung um eine durchaus umfangreiche, aber lohnende Aufgabe, die im Idealfall im Team in dafür durch die Schulleitung eingeräumten Teamzeiten geschieht. Im Sinne einer learner-centredness (Haß 2006: 22) sollte somit die Auswahl der Lerninhalte bzw. die Umsetzung vorgegebener Inhalte möglichst konkret an lebensweltlichen Erfahrungen der Lerner ausgerichtet und auf ihr Vorwissen bezogen sein, alters- und entwicklungsgemäß präsentiert werden und in der (emotionalen und kognitiven) Reichweite der Schülerinnen und Schüler liegen. Wie in den Ausführungen zur Didaktik des Förderschwerpunkts geistige Entwicklung angeführt, sollen Inhalte für die Lernenden Gegenwarts- und Zukunftsbedeutung besitzen.

Hinsichtlich der Lernumgebung sollte es sich gerade in heterogenen Settings um eine wie im CEFR angestrebte rich learning enviroment handeln, also eine Lernumgebung, die durch vielfältige Zugänge gekennzeichnet ist (Trim 2009: 21). Hierbei sind neue Medien und unterschiedliche Informationsquellen genauso eingeschlossen wie ein reiches Text- und Materialangebot, zum Beispiel in Form von echten Kommunikationsanlässen.

Entscheidend für den Anteil der Handlungsorientierung in inklusiven Settings ist die Wabl der Methoden: hier muss der Lehrende kompetent ein Repertoire bedienen und die Methoden auswählen, die handelndes Lernen zulassen oder sogar voraussetzen. Um den Anteil an kommunikativen Situationen für alle Lerner gleichzeitig hoch zu halten, eigenen sich vor allem Methoden des Kooperativen Lernens oder allgemein Arbeitsformen, die kommunikative Situation schaffen, wie es beispielsweise im projektartigen Unterricht der Fall ist. 
Ein weiterer zentraler Faktor für das Gelingen einer inklusiven Englischdidaktik bzw. eine Annäherung daran ist in der Lehrperson selbst in ihrer gesamten Lehrerpersönlichkeit zu sehen. Inklusion ist abhängig von Einstellungen und Normen der Gesellschaft und ihrer Mitglieder. Auf Unterrichtende kommen vielfältige Aufgaben in der Vorbereitung einer solchen Lernumgebung und Aufarbeitung der individuellen Voraussetzungen zu, welche beispielsweise Kenntnisse in bzw. die Bereitschaft zur Einarbeitung in Leistungs- und Entwicklungsdiagnostik voraussetzen. Die Planung der Lehrersprache als zentrales sprachliches Vorbild ist zu beachten (vgl. Steudle 2013: 9).

Für die konkrete Unterrichtsgestaltung des Englischunterrichts an inklusiven Schulen sollten jeweils für Schülergruppen „maßgeschneiderte“ Methoden der Differenzierung gesucht werden, die zum Beispiel den Anteil der visuellen Unterstützung in der gesprochenen Kommunikation durch Bilder, Piktogramme, Gebärden, einen bewussten Anteil von Schriftsprache berücksichtigen (vgl. Steudle 2013: 7f.). Der Unterricht muss Lernenden ausreichend Gelegenheiten bieten, für sie bedeutsame Inhalte auszuhandeln und echte Kommunikationsanlässe zu schaffen. Die Frage des vorwiegenden Einsatzes der Zielsprache Englisch muss entsprechend der wachsenden Heterogenität der Schülerinnen und Schüler ebenfalls vielfältiger werden: im Sinne einer aufgeklärten Einsprachigkeit muss der Anteil von Englisch als Unterrichtssprache wachsen und durch Nutzung von Techniken wie der „Sandwich-Technik“ unterstützt werden (vgl. Butzkamm 2007: 15). Weitere veranschaulichende Medien wie reale Gegenstände, Fotos, Bildkarten, Einsatz von Gestik und Mimik und wiederkehrenden Phrasen erleichtern das Verstehen aller Schülerinnen und Schüler (vgl. Steudle 2013: 7).

\section{Herausforderungen des inklusiven Englischunterrichts}

Experten und Interessierte aus Universität, Lehrerausbildung und Schule diskutierten im Rahmen verschiedener Workshops der Fachtagung „Englisch Inklusiv“ im Dezember 2013 über die Chancen und Möglichkeiten von Englisch in heterogenen Lerngruppen. Besonders im Austausch über Englischunterricht mit Schülerinnen und Schülern mit sogenannter geistiger Behinderung wurden wiederholt generelle Zweifel an der Lebensbedeutsamkeit von Englisch sowie Unsicherheiten zur Machbarkeit deutlich. Dieser 
Diskussionspunkt weist darauf hin, dass sich die für Inklusion notwendige gesellschaftliche Haltung, in der eine Person zunächst als Individuum gesehen und nicht zwischen geeignetem und ungeeignetem Inhalt unterschieden wird, noch in den Anfängen befindet. Insgesamt anzuerkennen ist jedoch die Tatsache, dass ein reger Austausch unter allen Teilnehmerinnen und Teilnehmern stattfand und somit der Thematik Inklusion eine gewisse Bedeutung zugemessen wurde.

Im Zuge der Auseinandersetzung wurde außerdem die Problematik der Entwicklungs- und Altersgemäßheit von Unterrichtsmaterial thematisiert, die beispielsweise jugendliche Sprachanfänger im Unterrichtsfach Englisch mit entwicklungsgemäß passendem aber altersungerechtem Material für die Grundschule konfrontiert. Der herrschenden Materialnot wird durch Verlage nur schrittweise und für Unterrichtende bis dahin mit einem enormen Aufwand zu begegnen sein.

Ein weiterer diskussionswürdiger Punkt von Englischunterricht im Rahmen zielgleicher und zieldifferenter Beschulung in inklusiven Settings ist im Spannungsverhältnis zwischen Lebensbedeutsamkeit und Lehrplan $\mathrm{zu}$ sehen. Der Lehrplan an allgemeinen Schulen ist, verbunden mit der Vorbereitung auf angestrebte Abschlüsse, die Basis des Unterrichts, während ein System wie die Förderschule geistige Entwicklung bei individuellen Bedürfnissen und Lebenswelten der Schülerinnen und Schüler ansetzen kann. Hier prallten Ansichten hinsichtlich des Ausgangspunkts von Unterrichtsplanung, des Selbstverständnis der Lehrerrolle und der Sinnhaftigkeit des Gemeinsamen Lernens aufeinander. Damit verbunden waren Forderungen an die Regierungen, Lehrpläne für verschiedene Schulformen soweit wie möglich zu öffnen und somit Raum zu lassen für individuelle Lernwege.

Die Leistungsbewertung/-kontrolle ist als weitere Herausforderung für den inklusiven Englischunterricht zu sehen. Laut AOSF ist eine individuelle Bezugsnorm für Schülerinnen und Schüler mit Förderbedarf anzulegen, bei der die Leistungen auf der Grundlage der im individuellen Förderplan festgelegten Lernziele beschrieben werden und die Leistungsbewertung sich auf die Ergebnisse des Lernens sowie die individuellen Anstrengungen und Lernfortschritte erstreckt. Dadurch wird die Forderung nach alternativen Formen der Leistungsbewertung hin zu individualisierenden Möglichkeiten deutlich. 


\section{Zusammenfassende Erkenntnisse und Forderungen an eine inklusive Didaktik}

Englischunterricht für alle ist eine Zielvorstellung, der sich nur in arbeitsintensiven Etappen und auf schulischer, schulorganisatorischer, universitärer, regierungsbezogener und gesellschaftlicher Ebene durch Schaffung entsprechender Rahmenbedingungen angenähert werden kann. Für Unterrichtende, die bereits vor der Herausforderung Inklusion stehen und für andere, die sich schrittweise auf den Weg dorthin machen, sind die folgenden drei Prinzipien für (Englisch-)Unterricht in heterogenen Gruppen als elementar anzusehen:

- Handlungsorientierung im Sinne einer bedeutsamen unterrichtlichen Interaktion unter Berücksichtigung individueller Interessen und Bedürfnisse in möglichst realen, sozialen Situationen

- Anerkennung von Differenz als Vielfalt und Differenzierung als Lösungsmöglichkeit in Form maßgeschneiderter Lernangebote praktizieren

- Individuelle Förderung als durchgängiges Unterrichtskonzept und konsequente Zielvorstellung durch Förderpläne für alle Schülerinnen und Schüler

Eine inklusive Schule muss Lehrende darin unterstützen, sich an unterschiedlichen Rahmen- und Lehrplänen zu orientieren, zwischen zielgleichen und zieldifferenten Bildungsabschlüssen zu differenzieren, diagnostische Prozesse zur Erhebung von Lernausgangslagen durchzuführen und Instrumente dazu kennenzulernen, Methoden der Binnendifferenzierung einzusetzen, Förderplanung als schulinternes Konzept zu erarbeiten, alternative Formen der Leistungsbewertung zu entwickeln, sich im Team interdisziplinär zu bereichern - kurz gesagt, den vielfältigen Anforderungen des inklusiven Unterrichtens professionell zu begegnen. Um diesen Prozess möglichst frühzeitig anzuleiten, ist in der Lehrerausbildung eine Vorbereitung für angehende Lehrerinnen und Lehrer in der universitären Ausbildung essentiell wichtig.

Aufgrund der Heterogenität von Schülerinnen und Schülern sind keine Patentrezepte zur gelungenen Inklusion möglich oder ertragreich. Deshalb bleibt für Unterrichtende abschließend der Hinweis, sich, trotz aller Skepsis und Unsicherheit, darauf zu besinnen, welche der genannten didaktischen Vorgehensweisen sie bereits intuitiv oder bewusst in ihrer erfolgreichen Arbeit im System Schule umsetzen. 


\section{Literaturverzeichnis}

Bach, Gerhard \& Timm Johannes-Peter (2013), Handlungsorientierung als Ziel und Methode. In: Bach, Gerhard \& Timm, Johannes-Peter (2013) (Hrsg.), Englischunterricht: Grundlagen und Methoden einer handlungsorientierten Unterrichtspraxis (5. überarbeitete Aufl.). Tübingen: Francke, 1-22.

Bleyhl, Werner (2000) (Hrsg.), Fremdsprachen in der Grundschule: Grundlagen und Praxisbeispiele. Hannover: Schroedel.

Butzkamm, Wolfgang (2007), Lust zum Lehren, Lust zum Lernen. eine neue Methodik für den Fremdsprachenunterricht (2. Aufl.). Tübingen: Francke. Haß, Frank (2006) (Hrsg.), Fachdidaktik Englisch. Tradition. Innovation. Praxis. Stuttgart: Klett.

Mühl, Heinz (1991), Einführung in die Geistigbehindertenpädagogik. Stuttgart: Kohlhammer.

Pitsch, Hans-Jürgen \& Thümmel, Ingeborg (2005), Handeln im Unterricht: Zur Theorie und Praxis des Handlungsorientierten Unterrichts mit Geistigbehinderten. Oberhausen: Athena.

Schulministerium NRW (2014), Ausbildungs- und Prüfungsordnungen aller Schulformen in Nordrhein-Westfalen [Online: https://www.schulministerium.nrw.de/docs/Recht/Schulrecht/APOen/index.html].

Steudle, Ines (2013), Wozu Englisch an der Schule mit dem Förderschwerpunkt geistige Entwicklung? Lernen Konkret 2013/1, 3-6.

Steudle, Ines (2013), Bewährte Rahmenbedingungen für den Englischunterricht. Lernen Konkret 2013/1, 7-8.

Straßmeier, Walter (2000), Didaktik für den Unterricht mit geistigbehinderten Schülern. München: Reinhardt.

Trim, John L. M. (2009), Gemeinsamer europäischer Referenzrabmen für Sprachen: Lernen, Lehren, Beurteilen. Berlin: Langenscheidt.

Ziemen, Kerstin (2012), Inklusion. In: Ziemen, Kerstin (Hrsg.) (2012), Inklusion Lexikon. [Online: http://www.inklusion-lexikon.de/Inklusion Ziemen.php]. 
Christiane M. Bongartz and Andreas Rohde - 978-3-653-97188-0

Downloaded from PubFactory at 01/11/2019 10:41:16AM

via free access 


\section{Roman Bartosch \& Andreas Köpfer \\ Stadtnatur als Gemeinsamer Gegenstand im inklusiven Englischunterricht - Spannungsfelder und Möglichkeiten in der didaktischen Fachdiskussion}

This article sets out to discuss current challenges and to describe possible solutions to some core problems of inclusive approaches to the teaching of English. While its main educational focus is on ways of dealing productively with the heterogeneity and diversity to be encountered not only in inclusive educational settings, special attention is paid to cognitive abilities and means of facilitating their development. 'Diversity' plays an important role both on the level of content and of methodology, which is why the article proposes a form of project work grounded in Feuser's notion of the 'common subject' ('Gemeinsamer Gegenstand'). This idea is developed in the form of a cooperative, activity-based task design for a project on 'urban nature'.

\section{Towards Inclusive English Language Teaching}

Inklusion bezieht sich auf die Analyse von Teilhabe- und Exklusionsprozessen in Bildungsinstitutionen und auf die Beschreibung der damit einhergehenden Transformation auf unterschiedlichen Ebenen des Bildungssystems (vgl. Wagner-Willi \& Sturm 2012; Werning 2014: 602). Sie findet zunehmend auch auf bildungspolitischer Ebene Beachtung und wird - unterstützt durch die UN-Behindertenrechtskonvention (UN 2006) - in normativ ausgerichteten Leitlinien terminologisch implementiert. Wenngleich öffentlich wahrgenommen, birgt dies die Gefahr, dass Inklusion eine leere, beliebig instrumentalisierbare Formel wird, unter der sich wahllose pädagogische und politische Praxen subsumieren (vgl. Hazibar \& Mecheril 2013). Das sich so ergebende Spannungsfeld konstituiert sich demnach zum einen als pädagogisch-normatives Projekt, in welches Hoffnungen und Ziele, wie z.B. der Abbau des dreigliedrigen und auf Leistungsselektion beruhenden Schulsystems, gelegt werden. Es äußert sich zum anderen allerdings auch in „DeSegregationsprozessen“ (vgl. Hinz 2013), und in dem damit verbundenen 
Abbau von und den Einsparungen bei Unterstützungsstrukturen sowie in einer hohen Beanspruchung von Lehrkräften.

Der Mangel an pädagogischen Konzepten jenseits der Forderung, ,es wollen zu müssen` wird also auf den Schultern der Lehrkräfte zur Quelle von Frustration und zu einem unauflösbarem Dilemma ihrer Praxis (s. dazu Amrhein \& Dziak-Mahler 2014: 11; Jacobs 2013: 52-53). Feuser attestiert daher dem Diskurs eine terminologische Diffusität: „Der Kontrollverlust der Pädagogik über die Begriffe Integration und Inklusion und ihre im Mainstream der Diskurse bestehende Unschärfe kennzeichnet ein hochgradiges fachwissenschaftliches Versagen“ (Feuser 2013: 27).

Um dem entgegenzuwirken, ist eine grundständige Aufarbeitung didaktischer und auch fachdidaktischer Fragestellungen notwendig, die den Kern der Überlegungen hinsichtlich inklusiver Bildung und Erziehung darstellen sollten, jedoch bisher nur rudimentär ins Blickfeld wissenschaftlicher Analysen gerieten (vgl. López Melero 2011: 38; Seitz 2006). Daher intendiert dieser Beitrag, am Beispiel inklusiver Englischdidaktik Möglichkeiten, aber auch Herausforderungen von Lern- und Bildungsprozessen im Kontext von Inklusion zu beschreiben. Inklusion wird dabei nicht als eindeutige Vorgabe verstanden, denn sie ist „kein fertiges Konzept, sondern [...] ein Diskursfeld, dessen Vermessung und Gestaltung bei weitem nicht abgeschlossen ist" (Roth 2014: 8; s. auch Armstrong, Armstrong \& Spandagou 2010: 3). Vor allem im Kontext der derzeit ebenfalls die Bildungslandschaft prägenden Diskussion um Kompetenzerwartungen und standardisierte Leistungsmessungen möchte dieser Beitrag bestehende Konflikte benennen und Lösungsansätze für einen inklusiven Englischunterricht skizzieren.

\section{Förderpädagogische Ausgangslage}

Wenngleich Exklusionsprozesse in Bezug auf unterschiedlichste Formen von Heterogenität betrachtet werden müssen, wird im Folgenden auf den Förderschwerpunkt Geistige Entwicklung fokussiert, der sich als schulspezifische Kategorie bzw. terminologische Klammer eines schwerlich zu rahmenden Personenkreises etabliert hat. Ebenso diffus zeigen sich die wenigen bzw. mitunter ausbleibenden Kompetenzerwartungen in Bezug auf den Fremdsprachenunterricht in diesem Förderschwerpunkt. So wird lediglich im niedersächsischen Kerncurriculum für den Förderschwerpunkt Geistige 
Entwicklung im Kompetenzbereich Kommunikation / Deutsch explizit formuliert:

Englisch prägt in vielfacher Form die Alltagswelt und -sprache von Kindern und Jugendlichen. Daher soll diese Sprachgegenwart im Unterricht bewusst gemacht und weiterentwickelt werden. Es ist angebracht, sprechenden Schülerinnen und Schülern mit bestimmten Voraussetzungen erste Kenntnisse der englischen Sprache für den Alltagsgebrauch zu vermitteln. (Niedersächsisches Kulturministerium 2007: 13)

Dennoch sind erste theoretische Annäherungen an den Fremdsprachenunterricht aus sonderpädagogischer Sicht erfolgt (vgl. Butzkamm 1998; v. Hebel \& Freye-Edwards 2012; Schick 2008; Schöler \& Degen 1999) und subsumiert (vgl. Köpfer 2014; 2015 i. Druck), da zunehmend Konsens herrscht, dass „das Fach Englisch ein bedeutsames Element der Allgemeinbildung darstellt, welches keiner Personengruppe vorenthalten werden darf“" (Köpfer 2014: 159).

Ein kohärenter didaktischer Rahmen, der eine theoretische Legitimierung der didaktischen Vorschläge als spezifisch sonderpädagogischen Zugang aufweist, ist allerdings nicht erkennbar. Es werden in erster Linie folgende didaktische Aspekte - auch für den Förderschwerpunkt Geistige Entwicklung - verhandelt:

- Schaffung einer anregenden und unterstützenden Lernumgebung

- Handlungsorientierung und auf Kommunikation beruhende Kooperation

- Lebensbedeutsamkeit und sprachliche Authentizität

- Verbindung von spielerischen Zugängen und Metakommunikation

- Nutzung von Anglizismen als immanenter Bestandteil des alltäglichen Sprachgebrauchs

Als übergeordnetes Ziel kann die Herausbildung einer fremdsprachlichen Handlungsfähigkeit bei SchülerInnen mit sonderpädagogischem Förderbedarf formuliert werden. Bezogen auf die Unterrichtsgestaltung wird im Allgemeinen die Hinwendung zu einer auf Individualisierung und Differenzierung basierenden Unterrichtsgestaltung postuliert, die zum Beispiel in Projektunterricht, Wochenplanarbeit oder weiteren Methoden des offenen Unterrichts durchgeführt werden kann (vgl. v. Hebel 2010; Kutscher 2003) und somit inhaltlich an Prämissen einer entwicklungslogischen Didaktik (Feuser 1995) anschließt, auf welche im späteren Verlauf eingegangen wird. 
Diese kann als konzeptuelle Brücke zwischen (sonder-)pädagogischen, fachdidaktischen wie bildungswissenschaftlichen Zugängen zu teilhabebasiertem Fremdsprachenunterricht fungieren, um zu einem vertieften Verständnis einer „inklusiven Englischdidaktik“ zu gelangen.

\section{Fachdidaktische Ressourcen und Möglichkeiten für eine inklusive Englischdidaktik}

Im Rahmen einer notwendigen didaktischen Aufarbeitung inklusiver Zielsetzung muss erkannt werden, dass die Herausforderung, Didaktik inklusiv weiterzuentwickeln, bereits durch zahlreiche Bemühungen in den Bereichen Differenzierung und Individualisierung, im Feld des interkulturellen Lernens sowie im Fokus vor allem der Literaturdidaktik auf Aspekte von Heterogenität angegangen wurde (Küchler \& Roters 2014; Nieragden 2014).

Wie Küchler \& Roters herausstellen, wird Inklusion in diesen Kontexten oft ,jedoch nicht als didaktisches Prinzip oder hinsichtlich denkbarer methodischer Umsetzungen näher erörtert" (Küchler \& Roters 2014: 236). Dies mag dem programmatischen Charakter der bisherigen Diskussion zum Thema geschuldet sein - es gilt jedoch, wie Nieragden (2014) betont, dass die in den letzten Jahren stattgefundene Veränderung englischdidaktischer Konzepte hin zu lernerzentriertem und somit individualisiertem Unterricht gerade durch das Medium Literatur leicht entsprechend angepasst werden können: „[D]ie Betonung der Individualität des Lese- und Verstehensprozesses, die Förderung emotionaler und empathischer Leserreaktionen [und] die Verdeutlichung der Perspektivenabhängigkeit des Verständnisses jeglicher in fiktionalen Texten vermittelten Information“ (Nieragden 2014: 167) bieten sich für diesen Perspektivwechsel ebenso an wie die Erkenntnis, dass ,Literatur' in diesem Kontext in erster Linie in einem weiten Sinn verstanden werden sollte und somit definiert wird als Textsorte, die „keinen rein informativen Zweck verfolgt, sondern narrativ eingesetzt wird [...] oder in ästhetisierter Form verwendet" (Albers 2014: 147) wird.

Diese Erkenntnis ist jedoch keineswegs auf fiktionale Texte im engeren Sinne oder literaturdidaktische Ziele begrenzt, sondern gilt für einen spielerischen Umgang mit Sprache allgemein. In jedem Fall sind Lernerzentrierung (vgl. Haß \& Kieweg 2012: 230), das Postulat der Bedeutsamkeit als zentrales Prinzip der Zielsprachhandlung (Haß 2013: 23), sowie 
produktionsorientierte Zugangsformen zur englischen Sprache, die das Sprachlernen mit kooperativen und sozialen Unterrichtsprinzipien koppeln, inzwischen allgemein anerkannt (vgl. Nünning \& Surkamp 2013: 151-160). Diese Erkenntnisse können auch im Kontext von Inklusion als zentrale Prinzipien des Englischunterrichts verstanden werden, um „authentische Sprachhandlungssituation zu schaffen und aufgabenorientiert zu unterrichten “ - Ulla Schäfer fasst dies, im Rückgriff auf Hicks, mit dem Slogan ,Create a Need‘ zusammen, der folglich auch im Kontext der Inklusion als Leitidee gelten mag (Schäfer 2014: 48-49).

\section{Differenzierung, Individualisierung, Lernziele und die konzeptuelle Rahmung durch Feusers Gemeinsamen Gegenstand}

Wie bereits angedeutet, ist es notwendig, vorhandene Potentiale für die Konzeption einer inklusiven Englischdidaktik zu nutzen. Diese Potentiale finden sich an zahlreichen Stellen (vgl. Albers 2014; Nieragden 2014; Schäfer 2014). Zusammenfassend lässt sich sagen, dass sie unter dem Oberbegriff der, Vielfalt' konzeptionell gebündelt werden können. Vielfalt meint dabei sowohl Methodenpluralität - hier sind vor allem Aspekte von Differenzierung und Individualisierung zu nennen - als auch Vielfalt als thematischen Fokalisierungspunkt - denn im Englischunterricht, und hier vor allem im Literaturunterricht, sind Aspekte des interkulturellen Lernens, des Perspektivwechsels und der Empathiefähigkeit zentral und können folglich entsprechend nutzbar gemacht werden.

Dabei ist eine Reflexion notwendig, in welchem Maße und zu welchem Zwecke Individualisierung eingesetzt werden kann, ob sie das alleinige gewinnbringende Ziel von Unterricht sein kann und wie mit der scheinbaren Antinomie von individualisiertem und gemeinschaftlichem Lernen proaktiv umgegangen werden kann (vgl. Scheidt \& Köpfer 2013). Auch wenn soziales Lernen und die Anbahnung kommunikativer Kompetenz gerade im interkulturellen und inklusiven Kontext von großer Bedeutung sind, darf nicht übersehen werden, dass die Institution Schule immer auch eine Allokations- und Enkulturationsfunktion ausübt, die zwingend auf die gemeinschaftliche Bearbeitung unterrichtlicher Fragestellungen angewiesen ist (vgl. Brodkorb 2013: 27). 
Im Folgenden wird auf das didaktische Modell der Arbeit am Gemeinsamen Gegenstand rekurriert, welches von Georg Feuser (vgl. 1995; 1998) entwickelt wurde und didaktische Instrumentarien bzw. Prämissen bereithält, die fruchtbar gemacht werden können als didaktisch-konzeptionelle Klammer für inklusiven Unterricht und für soziales Lernen im Sinne von Kooperation - im späteren Verlauf am Beispiel „Stadtnatur“ expliziert. Ohne an dieser Stelle auf die wissenschaftstheoretischen Wurzeln der entwicklungslogischen Didaktik eingehen zu können (vgl. Feuser 1995), kann als leitendes Postulat subsumiert werden, dass „alle Kinder in Kooperation miteinander auf ihrem jeweiligen Entwicklungsniveau und mittels ihrer momentanen Denk- und Handlungskompetenzen an und mit einem Gemeinsamen Gegenstand lernen und arbeiten“ (Feuser 1998: 177) sollen - umgesetzt im Rahmen von Projektarbeit. Der Gemeinsame Gegenstand stellt dabei nicht nur den inhaltlich übergeordneten Bezugspunkt dar, sondern kann als Prozesskategorie verstanden werden, an den die kooperativen Tätigkeiten der SchülerInnen ausgerichtet sind. Durch die Bearbeitung einer gemeinsam zu lösenden Aufgabe wird die Zusammenarbeit von SchülerInnen auf verschiedenen Schwierigkeitsniveaus und unter Bezugnahme auf unterschiedliche Talente und Fähigkeiten gelebte Unterrichtspraxis; der Gemeinsame Gegenstand ist also gleichermaßen inhaltlicher Fokus als auch zentrale Sozialform.

\section{Aufgabendesign am Beispiel eines Projekts zum Thema ,Stadtnatur ${ }^{6}$}

Im Sinne einer fachdidaktischen Ausrichtung, die im Englischunterricht mehr sieht „als nur die Vermittlung von fremdsprachlichen Kompetenzen“, nämlich „die Erziehung zu Toleranz und freiem Denken sowie die Wertschätzung des Individuums, der Natur, der Heimat sowie fremder Kulturen “ (Volkmann 2012: 393), bietet sich der Themenkomplex „Umgang mit der Natur [...] angesichts sich weltweit auswirkender Natur- wie Technikkatastrophen, der Erderwärmung, dem Schwinden der natürlichen Ressourcen sowie der anhaltenden Umweltzerstörung mit verheerenden Folgen“ mehr als an (ebd.: 394) und kann auf eine lebhafte Diskussion in der didaktischen Forschung zurückgreifen (vgl. Bartosch \& Grimm 2014; Garrard 2012; Küchler 2011; Mayer \& Wilson 2006; Matthewman 2011). Gleichzeitig steht man bei der 
Behandlung dieses Themas vor dem Problem der schier unbeherrschbaren Komplexität, wie die von Volkmann angeführte Liste zeigt: das Thema ist nicht nur wissenschaftlich, ökonomisch und ökologisch höchst komplex, sondern gerade in seiner Abstraktion, eingeschränkten Sichtbarkeit und seinem narrativ nur schwer darstellbaren Wesen eine besondere Herausforderung für den schulischen (Englisch-)Unterricht.

Sasha Matthewman argumentiert daher dafür, die ökologisch-systemische Natur der Stadt in den Augenschein zu nehmen:

$[C]$ hildren $[\ldots]$ inhabit a predominantly urban culture. Children in urban schools need to be supported to consider their own environment as relating to concepts of nature and sustainable living. English teaching can be an important source for such reflection. (Matthewman 2011: 109)

Folgt man dieser Argumentation, lässt sich die Behandlung des Themenkomplexes (Stadt-)Natur im Kontext der bis einschließlich 2015 andauernden UN-Dekade für nachhaltige Entwicklung verorten, und (Stadt-) Natur kann als Einstieg für das, mit Klafki gesprochen, „epochaltypische [...] Schlüsselproblem“ (Klafki 1991: 50) der Mensch-Umwelt-Beziehungen betrachtet werden.

Um als Lehrkraft den Herausforderungen von Heterogenität Rechnung zu tragen, bietet sich an, diese primär durch ein entsprechend durchdachtes und an den Bedürfnissen der SchülerInnen orientiertes Aufgabendesign $\mathrm{zu}$ fokussieren. Wir wollen uns daher exemplarisch auf den für den Englischunterricht zentralen Aspekt der Aufgaben (tasks) konzentrieren. Wie Ulla Schäfer herausstellt, ermöglichen vor allem halboffene oder offene Aufgabenformate ,individuelle Zugänge und [ermöglichen so] Lernfortschritte“ (Schäfer 2014: 50), weil es bei diesen Aufgaben nicht um die eine, korrekte Lösung geht, sondern um individuelle Lösungen zu einem übergeordneten Aufgabenkomplex. Wie Schäfer ebenfalls herausstellt, bedeuten offene und halboffene Aufgabenformate jedoch häufig, dass die Lehrkraft verstärkt vom Lehrwerk, das eher geschlossene Aufgabeformate anbieten wird, absehen und eigene Aufgaben konstruieren muss, was viele Lehrerinnen und Lehrer zunächst abschreckt (Schäfer 2014: 51). Hier besteht ein Handlungsbedarf aufseiten der Schulbuchverlage, die jedoch zum Beispiel mit den vom Finken-Verlag herausgegebenen Modulpaketen Early Bird und Little Early Bird erste Schritte in diese Richtung unternommen haben. 
Die Notwendigkeit eigenständiger Gestaltung bietet gleichzeitig die Möglichkeit, lernerzentrierten Unterricht durchzuführen: Die Lehrkraft kann zum Beispiel in einer Einstiegsrunde zum Thema Stadtnatur ein Wimmelbild einer Stadtlandschaft zeigen und Begriffe nennen lassen, die die SchülerInnen bereits kennen. Diese Form eines Einstiegs mittels eines „stummen Impulses“ ist nicht nur ein Sprechanlass für alle SchülerInnen, sondern er offenbart der Lehrkraft auch, „mit welchen Interessensgruppen [man] es in [der] Lerngruppe zu tun" hat, wie Schäfer herausstellt (2014: 49). Das auf diesem Einstieg aufbauende Aufgabendesign ist somit nicht bloß von der Lehrperson durchdacht, sondern direkt an den Bedürfnissen der Lernenden orientiert, die die entsprechenden Informationen und Impulse einzubringen aufgefordert worden sind. Möchte man anhand dieser Informationen nun eine entsprechende Aufgabe oder Unterrichtsreihe konzipieren, ist es sinnvoll, sich die von Jane Willis (1996) vorgestellten Phasen eines tasks in Erinnerung zu rufen: demnach folgt auf eine pre-task-Phase, in der das Thema vorgestellt und notwendige Informationen gegeben werden, ein sog. task cycle, der aus kleineren Einzelaufgaben, dem LehrerInnen-Feedback sowie Ergebnissicherung besteht. Abschließend verhandelt ein dem language focus gewidmetes Segment die metalinguistischen Lerninhalte und sichert diese Ergebnisse (zit. nach Schäfer 2014: 52-53).

Aufgrund der Verortung unserer Ausführungen im inklusiven Unterricht wird ein Projekt mit den SchülerInnen skizziert, zu dessen Bearbeitung in erster Linie gehört, aufmerksam die eigene Umwelt wahrzunehmen und ganzheitlich zu erfahren: „Where can you find nature in your city? “ „What is your favourite spot in your home town?" „Do you know the names of flowers, trees and animals around you? “. Diese Entdeckungsreise bildet die Basis für eine auf der individuellen Wahrnehmung beruhende Exploration sowohl der eigenen Umgebung als auch des fremdsprachlichen Vokabulars dieser Umgebung, denn das von der Lehrkraft schließlich vorgeschlagene Ziel der Arbeit soll sein, eine Stadtführung zum Thema Natur zu organisieren.

Damit ist zum Thema Stadtnatur ein Gemeinsamer Gegenstand im Sinne eines - sinnbildlich durch die Stadtführung verdeutlicht - gemeinsamen Lernprozesses angestoßen, und die Differenzierung kann nun auf der Ebene des task cycle stattfinden. Es können in kooperativer Arbeit der SchülerInnen Wörter gefunden und notiert werden, Bilder und Skizzen 
werden benötigt; es bedarf eines Plans der Stadt sowie der Erstellung eines Fahrplans - Elemente also aus dem lebenspraktischen und lebensbedeutsamen Kontext der SchülerInnen; dazu wiederum bedarf es Adjektiven und Präpositionen, chunks und auch literarischer Texte. Letzteres bedeutet ja keinesfalls, dass abstrakte Analysen verlangt werden sollen, sondern dass ein wie oben erwähnter spielerischer Umgang mit Sprache, Metaphern und poetischen Beschreibungen motivierend und gewinnbringend eingesetzt werden kann (vgl. Matthewman 2011: 118).

Schließlich ist die Planung einer solchen Führung, die entweder mit anderen SchülerInnen durchgeführt werden könnte oder aber im Kontext möglicher Projekte zur Nachhaltigkeit, die derzeit immer in verschiedenen Kontexten angeboten werden, das zentrale Element der praktischen bzw. handlungsorientierten Anwendung; auch hier lässt sich eine Vielzahl von offenen und halboffenen, auf Kommunikation beruhenden Aufgabenformaten finden, die der Heterogenität der SchülerInnen gerecht werden können, und auch hier lässt sich der language focus den spezifischen (Teil-)Kompetenzerwartungen entsprechend variieren, von Vokabel- und chunk-Listen über die Erstellung von Fahrplänen bis hin zur Gestaltung von Informationsbroschüren und Postern. Damit ist sowohl der im engeren Sinne fachdidaktische Fokus des Sprachenlernens und der kooperativen Lernformen erfüllt, als auch ein Stück transkulturellen Verstehens der Bedeutung von Stadtnatur vor allem der Existenz von Natur in der Stadt, die immer eklatant aber doch fast unsichtbar auf natürliche Ressourcen angewiesen ist - behandelt worden, was sich im Kontext von Erziehung zur Nachhaltigkeit als zentrales Lernziel formulieren ließe (vgl. Zinn 2013, v.a. 366-367).

\section{6. „Stadtnatur“ als komplexe Kompetenzaufgabe im inklusiven Englischunterricht}

Der Kompetenzbegriff nimmt aktuell eine prädominante Rolle in der erziehungswissenschaftlichen Fachdiskussion ein. An ihm macht sich ein Spannungsfeld zwischen schulischen Ansprüchen an bildungsgerechtes und soziales Lernen (wie es in Arbeiten zur Inklusion dargestellt wird) und dem gleichzeitigen Drang nach messbaren, standardisierten Kompetenzen fest, die das humanistisch-idealistische Konzept der ,Bildung' durch einen individualisierten Leistungsbegriff endlich ablösen mögen. Bislang gibt es 
wenige Arbeiten hinsichtlich eines Verständnisses von Kompetenz, das nicht im Widerspruch zur Inklusion steht, sondern womöglich für diese fruchtbar gemacht werden kann (vgl. Ziemen 2013).

Um sowohl dem Kompetenzbegriff als auch der Vorgabe, Kommunikation nicht als simplen output sondern als Diskursteilhabe zu verstehen, gerecht zu werden, schlägt Hallet das Konzept der „komplexen Kompetenzaufgabe" vor:

$\mathrm{Zu}$ ihrer Bewältigung müssen die Schülerinnen und Schüler problemlösende Strategien sowie kognitive, sozial-interaktionale und diskursive Fähigkeiten anwenden und (weiter-)entwickeln [...]. Kompetenzaufgaben initiieren daher nach dem Vorbild lebensweltlicher Herausforderungssituationen komplexe Interaktionen und Aushandlungsprozesse im Klassenzimmer [...]. (Hallet 2012: 11)

Trotz der hier explizierten hohen Anforderung ist es gerade die Komplexität dieser Aufgaben, die ein gemeinsames und somit letztlich potentiell inklusives Lernen erforderlich macht. Der Gemeinsame Gegenstand, also die gemeinsam ausgehandelte Themen- und Inhaltsorientierung, ist dabei als Fokus zentral. Auch wenn die Formulierung von Kompetenzen im Sinne einer Reduzierung auf Fertigkeiten damit unmöglich gemacht wird - was im eingangs kritisierten institutionellen Rahmen und im Kontext der derzeit vorherrschenden Orientierung am linearen output, der im Kontext von Kultur- und Literaturdidaktik allgemein und im Kontext von ökologisch orientiertem Unterricht erst recht jeglichen Sinnes beraubt ist (vgl. Bartosch \& Garrard 2014; Gerhardt 2006) - so lässt sich dagegen jedoch klar zeigen, dass eine der Kompetenzen, die neben Spracherwerb und dem erfolgreichen Umgang mit dem speziellen task erworben wurde, die der Kooperation ist. Dies ist sowohl im inklusiven Unterricht bedeutsam als auch ein zentrales Anliegen jeglicher Erziehung zur Nachhaltigkeit.

\section{Literaturverzeichnis}

Albers, Carsten (2014), Englisch an der Förderschule: Literaturdidaktische Perspektiven. In: Bartosch, Roman \& Rohde, Andreas (Hrsg.) (2014), 147-156.

Amrhein, Bettina \& Dziak-Mahler, Myrle (2014), Fachdidaktik Inklusiv. Eine Aufgabe für die LehrerInnenbildung der Zukunft. In: Amrhein, Bettina \& Dziak-Mahler, Myrle (Hrsg.) (2014), 11-13. 
- (Hrsg.) (2014), Fachdidaktik Inklusiv. Auf der Suche nach didaktischen Leitlinien für den Umgang mit Vielfalt in der Schule. Münster \& New York: Waxmann.

Armstrong, Ann Cheryl; Armstrong, Derrick \& Spandagou, Ilektra (2010), Inclusive Education. International Policy and Practice. Los Angeles: Sage.

Bartosch, Roman \& Garrard, Greg (2013), The Function of Criticism. A Response to William Major and Andrew McMurry's Editorial. Journal of Ecocriticism 5: 1, 1-6.

Bartosch, Roman \& Grimm, Sieglinde (Hrsg.) (2014), Teaching Environments. Ecocritical Perspectives. Frankfurt am Main: Peter Lang.

Bartosch, Roman \& Rohde, Andreas (Hrsg.) (2014), Im Dialog der Disziplinen. Englischdidaktik - Förderpädagogik - Inklusion. Trier: Wissenschaftlicher Verlag.

Brodkorb, Mathias (2013), Warum Inklusion unmöglich ist. Profil (April), 25-34.

Butzkamm, Wolfgang (1998), Zehn Prinzipien des Fremdsprachenlernens und -lehrens. In: Timm, Johannes-Peter (Hrsg.) (1998), Englisch lernen und lehren. Berlin: Cornelsen, 45-52.

Deneke, Sandra (2010), An einem Strang ziehen - für ein lebensbedeutendes Fach. Praxis Fördern 1, 44-45.

Feuser, Georg (1995), Behinderte Kinder und Jugendliche - zwischen Integration und Aussonderung. Darmstadt: Wissenschaftliche Buchgesellschaft.

Feuser, Georg (1998), Aspekte einer Didaktik unter Berücksichtigung tätigkeitstheoretischer und entwicklungspsychologischer Erkenntnisse. In: Eberwein, Hans (Hrsg.) (1998), Behinderte und Nichtbehinderte lernen gemeinsam. Handbuch der Integrationspädagogik. Weinheim: Beltz, 170-179.

Feuser, Georg (2013), Inklusive Bildung - ein pädagogisches Paradoxon. In: Banse, Gerhard \& Meier, Bernd (Hrsg.) (2013), Inklusion und Integration. Theoretische Grundfragen und Fragen der praktischen Umsetzung im Bildungsbereich. Frankfurt am Main: Peter Lang, 25-41.

Garrard, Greg (Hrsg.) (2012), Teaching Ecocriticism and Green Cultural Studies. Basingstoke: Palgrave Macmillan. 
Gerhardt, Christine (2006), Literature, Nature, and the Crux of Consciousness-Raising. In: Mayer, Sylvia \& Wilson, Graham (Hrsg.) (2006), Ecodidactic Perspectives on English Language, Literatures and Cultures. Trier: Wissenschaftlicher Verlag, 223-233.

Hallet, Wolfgang (2012), Die komplexe Kompetenzaufgabe. Fremdsprachige Diskursfähigkeit als kulturelle Teilhabe und Unterrichtspraxis. In: Hallet, Wolfgang \& Kramer, Ulrich (Hrsg.) (2012), Kompetenzaufgaben im Englischunterricht. Grundlagen und Unterrichtsbeispiele. Seelze: Klett, 8-19.

Haß, Frank (2013), Fachdidaktik Englisch. Tradition Innovation Praxis. Stuttgart: Klett.

Haß, Frank \& Kieweg, Werner (2012), I can make it! Englischunterricht für Schülerinnen und Schüler mit Lernschwierigkeiten. Seelze: Friedrich.

Hazibar, Kerstin \& Mecheril, Paul (2013), Es gibt keine richtige Pädagogik in falschen Verhältnissen. Widerspruch als Grundkategorie einer Behinderungspädagogik. Zeitschrift für Inklusion-online.net 1 [Online: http://www.inklusion-online.net/index.php/inklusion-online/article/ view/23/23. 10.02.2015].

v. Hebel, Anja (2010), „Yes, we can talk!“ Englisch für Jugendliche mit sonderpädagogischem Förderbedarf. Praxis Fördern 1, 4-6.

v. Hebel, Anja \& Freye-Edwards, Elke (2012), Heterogenität verstehen Individualität fördern. „Individuals making a difference“ im Englischunterricht. Praxis Fördern 1, 6-8.

Hinz, Andreas (2013), Inklusion - von der Unkenntnis zur Unkenntlichkeit?!. Zeitschrift für Inklusion-online.net 1 [Online: http://www inklusion-online net/index.php/inklusion-online/article/view/26/26. 10.02.2015].

Jacobs, Kurt (2013), Inklusion als menschenrechtlicher Baustein für umfassende soziale Partizipation. In: Banse, Gerhard \& Meier, Bernd (Hrsg.) (2013), Inklusion und Integration. Theoretische Grundfragen und Fragen der praktischen Umsetzung im Bildungsbereich. Frankfurt am Main: Peter Lang, 43-58.

Klafki, Wolfgang (1991), Neue Studien zur Bildungstheorie und Didaktik: Zeitgemäße Allgemeinbildung und kritisch-konstruktive Didaktik. Weinheim und Basel: Beltz. 
Köpfer, Andreas (2014), Kernkategorien einer inklusiven Englischdidaktik. In: Bartosch, Roman \& Rohde, Andreas (Hrsg.) (2014), 157-166.

Köpfer, Andreas (2015), Zielperspektive: Inklusiver Englischunterricht - didaktische Diskussion am Unterrichtsbeispiel „London“. In: Musenberg, Oliver \& Riegert, Judith (Hrsg.) (im Druck), Inklusiver Fachunterricht in der Sekundarstufe. Stuttgart: Kohlhammer.

Küchler, Uwe (2011), Linking Foreign Language Education and the Environment: Intercultural Communicative Competence and Environmental Literacy. In: Oppermann, Serpil; Özdak, Ufuk; Özkan, Nevin \& Slovic, Scott (Hrsg.) (2011), The Future of Ecocriticism: New Horizons. Cambridge: Cambridge Scholars Publishing, 436-452.

Küchler, Uwe \& Roters, Bianca (2014), Embracing Everyone: Inklusiver Fremdsprachenunterricht. In: Amrhein, Bettina \& Dziak-Mahler, Myrle (Hrsg.) (2014), 233-248.

Kutscher, Sandra (2003), Englisch im Förderschwerpunkt Lernen. Zeitschrift für Heilpädagogik 12, 511-517.

López Melero, Miguel (2011), Träume einer Gesellschaft der Inklusion. Behinderte Menschen 6, 32-43.

Matthewman, Sasha (2011), Teaching Secondary English as if the Planet Matters. London \& New York: Routledge.

Mayer, Sylvia \& Wilson, Graham (Hrsg.) (2006), Ecodidactic Perspectives on English Language, Literatures and Cultures. Trier: Wissenschaftlicher Verlag.

Niedersächsisches Kultusministerium (Hrsg.) (2007), Kerncurriculum für den Förderschwerpunkt Geistige Entwicklung. [Online: http://db2.nibis. de/1db/cuvo/datei/kc foe geistige nib.pdf. 10.02.2015].

Nieragden, Göran (2014), Literaturdidaktik war eigentlich immer schon inklusiv - Ein Plädoyer für den Einsatz von kleineren fiktionalen Textformen im Englischunterricht förderpädagogischer und inklusiver Klassen. In: Bartosch, Roman \& Rohde, Andreas (Hrsg.) (2014), 167-182.

Nünning, Ansgar \& Surkamp, Carola (2013), Text - Literatur - Kultur: Handlungs- und produktionsorientierter Literaturunterricht. In: Bach, Gerhard \& Timm, Johannes-Peter (Hrsg.) (2013), Englischunterricht. Tübingen und Basel: Francke, 148-171.

Roth, Joachim (2014), Vorwort. In: Amrhein, Bettina \& Dziak-Mahler, Myrle (Hrsg.) (2014), 7-9. 
Schäfer, Ulla (2014), Englischunterricht für Schülerinnen und Schüler mit Lernschwierigkeiten. In: Bartosch, Roman \& Rohde, Andreas (Hrsg.) (2014), 46-62.

Scheidt, Katja \& Köpfer, Andreas (2013), Die Antinomie Individualisiertes $\&$ Gemeinsames Lernen - Versuch einer Strukturierung. In: Dorrance, Carmen \& Dannenbeck, Clemens. (Hrsg.) (2013), Doing Inclusion - Inklusion in einer nicht inklusiven Gesellschaft (Tagungsband zur Jahrestagung der Inklusionsforscher/innen). Bad Heilbrunn: Klinkhardt, 217-225.

Schick, Frauke (2008), „Clothes from head to toe. Let's get dressed!” Projektorientierter Englischunterricht mit sogenannten geistig behinderten Schülern. Behindertenpädagogik 4, 428-437.

Schöler, Jutta \& Degen, Sabrina (1999), Integration im Englischunterricht. Chancen gemeinsamen Lernens für Kinder mit und obne Behinderung. Neuwied, Kriftel, Berlin: Luchterhand Verlag.

Seitz, Simone (2006), Die Frage nach dem „Kern der Sache“. Zeitschrift für Inklusion-online.net 1 [Online: http://www.inklusiononline.net/index. php/inklusion/article/view/15/15,22.02.2014].

UN - United Nations (2006), UN-Convention on the Rights of Persons with Disabilities. [Online: http://www.un.org/disabilities/convention/ conventionfull.shtml. 22.02.2014].

Volkmann, Laurenz (2012), Ecodidactics als Antwort auf die planetarische Bedrohung? Zum Einsatz von Ecopoetry im Englischunterricht. In: Hammer, Julia; Eisenmann, Maria \& Ahrens, Rüdiger (Hrsg.) (2012), Anglophone Literaturdidaktik. Zukunftsperspektiven für den Englischunterricht. Heidelberg: Winter, 393-408.

Wagner-Willi, Monika \& Sturm, Tanja (2012), Inklusion und Milieus in schulischen Organisationen. Zeitschrift für Inklusion-online.net 4 [Online: http://www.inklusion-online.net/index.php/inklusion/article/ view/185/173. 10.02.2015].

Werning, Rolf (2014), Stichwort: Schulische Inklusion. Zeitschrift für Erziehungswissenschaft 17: 4, 601-623.

Willis, Jane (1996), A Framework for Task-Based Learning. London: Longman.

Ziemen, Kerstin (2013), Kompetenz für Inklusion. Göttingen: Vandenhoeck \& Ruprecht.

Zinn, Sascha (2013), Bildungsziel Nachhaltigkeit!? Eine interdisziplinäre Reflexion. Frankfurt am Main: Peter Lang. 


\section{Lena Kläser \& Andreas Rohde \\ Fremdsprachenunterricht für gehörlose Schülerinnen und Schüler am Beispiel des Unterrichtsfaches Englisch}

Due to the number of languages that are involved, second/foreign language teaching presents a particular challenge for deaf children at German special needs schools and in the inclusive second/foreign language classroom. Many deaf children are born to hearing parents and, therefore, have not been exposed to German Sign Language since birth. At school entry, written German is introduced, which can be regarded as a distinct language for the deaf for two reasons. Firstly, it represents spoken German, which can only be partially decoded by the deaf through lip reading. Secondly, the syntax of German Sign Language differs from that of spoken/ written German. In addition, these deaf children are introduced to English in its spoken and written forms and, at least theoretically, to British or American Sign Language if these are available. This paper focuses on the complexity of situations in which further languages and, correspondingly, different modalities, are involved in the second/foreign language classroom.

\section{Altersgemäßer Spracherwerb}

Der altersgemäße Erwerb der Muttersprache (L1) ist eine wichtige Voraussetzung für jedes weitere Sprachenlernern (Siebert-Ott 2001). Das gilt für hörende ebenso wie für gehörlose Kinder (Haug 2009: 102). Um einen genaueren Blick auf gehörlose Kinder, die in Deutschland Englisch als Fremdsprache im Englisch-Unterricht lernen sollen, werfen zu können, ist es daher bedeutsam, sich den L1-Erwerb dieser Kinder anzuschauen. Es werden hier zwei Populationen unterschieden: 1. Gehörlose Kinder, deren Eltern gehörlos sind, 2. Gehörlose Kinder, deren Eltern hörend sind. Letztere Konstellation trifft auf $95 \%$ der gehörlosen Kinder in Deutschland zu (Haug 2009: 23).

Die Kinder hörender Eltern erwerben eine Gebärdensprache meist erst im Kindergarten oder in der Schule. Dies bedeutet, dass eine verlässliche L1 für diese Kinder nicht angenommen werden kann, was möglicherweise erhebliche Konsequenzen für jeden weiteren Spracherwerb bedeutet. Die 
erste Frage, die wir in diesem Beitrag stellen, ist daher, wie gewährleistet werden kann, dass alle gehörlosen Kinder eine altersangemessene L1 erwerben - ungeachtet der Hörfähigkeit der Eltern. Zu diesem Zweck werden vier Ansätze diskutiert, die sich in der Sichtweise unterscheiden, wann den Kindern welche Sprache (Gebärden- und/oder Lautsprache) angeboten wird:

- die sukzessive Methode ${ }^{1}$

- das schwedische Modell

- Total Communication

- das bilinguale Konzept / die Parallele Zweisprachigkeit

Die Modelle werden dahingehend geprüft, inwieweit sie in der Lage sind, die besondere Rolle gehörloser Menschen in der hörenden Mehrheitsgesellschaft zu berücksichtigen.

Die zweite wesentliche Frage, die in diesem Beitrag diskutiert wird, ist die nach der Fremdsprache, die die Kinder im Unterricht lernen sollen. Ist es sinnvoll, Englisch als Laut- oder Schriftsprache zu lehren - oder müsste American Sign Language (ASL) oder British Sign Language (BSL) Unterrichtsgegenstand sein - oder gar eine Kombination der genannten Varianten?

\section{Theoretischer Hintergrund}

Zunächst ist es erforderlich, einige für den Beitrag bedeutsame Termini zu definieren, u.a. muss zwischen „Hörschädigung “, „Schwerhörigkeit“ und „Gehörlosigkeit“ unterschieden werden. Um den L1-Erwerb einer Gebärdensprache mit dem L1-Lauterwerb vergleichen zu können, ist eine kurze Charakterisierung von Gebärdensprachen notwendig. Es wird verdeutlicht, dass sich Gebärdensprachen keineswegs als „impoverished, deficient, or ungrammatical“ (Coleman 2006: 471) charakterisieren lassen.

1 Die hier referierten Ansätze werden in der Terminologie wiedergegeben, wie sie in der Literatur verwendet wird. Daher erscheint der Terminus „sukzessive Methode“, aber „das schwedische Modell“ bzw. „das bilinguale Konzept“. Streng genommen handelt es sich hier um verschiedene Ansätze, Laut- und Gebärdensprache(n) einzusetzen. 


\section{Terminologie: Hörschädigung - gehörlos - schwerhörig}

„Hörschädigung” ist der generische Terminus für einen vollständigen oder teilweisen Verlust der Hörfähigkeit (WHO 2010). Liegt eine Hörschädigung vor, bedeutet das, dass im Hörorgan, der Hörbahn oder in den Hörzentren eine Störung vorliegt, die prä-, peri-, postnatal oder im Erwachsenenalter entstanden sein kann (Hausen 2013: 96). Zu den Arten der Hörschädigung gehören die Schalleitungsschwerhörigkeit, die sensorineurale Schwerhörigkeit, die kombinierte Schallleitungs-Schallempfindungsschwerhörigkeit und die Gehörlosigkeit (Leonhardt 2002: 50f.). Bei der Gehörlosigkeit handelt es sich nicht um eine eigene Art der Hörschädigung, sondern um eine hochgradige Schallempfindungsschwerhörigkeit. Man spricht von Taubheit, wenn diese so gravierend ist, dass überhaupt keine Hörreste vorliegen - dieser Fall ist sehr selten. In den überwiegenden Fällen liegen auch bei einer Gehörlosigkeit demnach Hörreste vor; sie reichen jedoch nicht aus, um auf natürlichem Wege, selbst nicht mit technischen Hilfsmitteln, Lautsprache auf der Schallebene wahrzunehmen (ebd.: 53f.). Gehörlosigkeit bedeutet, dass die Hörschwelle im Hauptsprachbereich über $90 \mathrm{~dB}$ liegt (bei normalhörenden Menschen $0 \mathrm{~dB}$ ). In diesem Beitrag beschäftigen wir uns ausschließlich mit Kindern, auf die diese Charakterisierung zutrifft.

\section{Gebärdensprachen sind natürliche Sprachen}

Gebärdensprachen sind in vielerlei Hinsicht wie gesprochene (Laut-)Sprachen. Sie sind natürliche Sprachen, die spontan entstehen, wo immer es eine „community of communicators“ (Sandler 2006: 328) gibt. Gebärdensprachen erfüllen alle sozialen und mentalen Funktionen von Lautsprachen, und sie werden ohne Instruktion von Kindern erworben - sofern normaler Kontakt und Interaktion gegeben sind (ebd.). Betrachtet man die in Coleman (2006: 471f.) zitierten 16 Merkmale, die Hockett und Altmann als Erkennungsmerkmale natürlicher Sprachen ansetzen, so trifft lediglich das erste Merkmal „use of the vocal-auditory channel“ nicht auf Gebärdensprachen $\mathrm{zu}$, da sie manuell-visuell bestimmte Systeme sind und damit ein anderes physikalisches Medium nutzen (Sandler 2006: 328). Dennoch wird auch in der Gebärdensprachenforschung der Terminus „Phonologie“ benutzt, um die Funktion der kleinsten bedeutungsunterscheidenden Einheiten zu 
beschreiben (Becker \& von Meyenn 2012). Alle weiteren Merkmale lassen sich auch auf Gebärdensprachen anwenden.

1. Gebärdensprachen sind produktiv, d.h., es gibt eine begrenzte Anzahl von kleinen Elementen, die zu größeren nicht begrenzten Elementen zusammengesetzt werden können. Diese kleinen Elemente sind die verschiedenen Gebärden und ihre Parameter. Im Jahr 1960 konnte Stokoe (1960) nachweisen, dass die drei phonologischen Hauptkategorien für Gebärdensprachen „Handform“, „Ausführungsstelle“ und „Bewegung“ sind. Battison (1978) fügte einen vierten Parameter hinzu, die „Handstellung“. Auf der Basis dieser nun vier Parameter lässt sich eine unbegrenzte Anzahl an Gebärden generieren. Gebärdensprachen wie auch Lautsprachen beruhen auf ähnlichen, möglicherweise sogar universellen Prinzipien. Sie sind hierarchisch aufgebaut und benutzen eine begrenzte Anzahl bedeutungsunterscheidender Einheiten, um bedeutungstragende lexikalische Einheiten in der Lautsprache und Gebärden in der Gebärdensprache - und, darauf aufbauend, Sätze zu bilden (Becker \& von Meyenn 2012: 32f.).

2. Gebärdensprachen sind diskret, d.h., die Zeichen/Gebärden sind eindeutig voneinander abgrenzbar. Die soeben erwähnten vier Parameter bilden die phonologischen Kategorien. So unterscheiden sich die Gebärden $\mathrm{NOT}^{2}$ und FRAGEN in der deutschen Gebärdensprache lediglich durch die Ausführungsstelle und bilden daher ein Minimalpaar. Die Handform (f-Handform: Berührung des Zeigefingers mit dem Daumen, Ausstrecken aller anderen Finger) und die Bewegung (vom Körper weg) sind gleich. Demnach macht die Ausführungsstelle den Bedeutungsunterschied aus. Wenn die Gebärde am Mund ausgeführt wird, bedeutet sie „fragen“; wenn sie hingegen am Hals ausgeführt bzw. gebärdet wird, bedeutet sie NOT (vgl. Becker \& von Meyenn 2012: 41f.).

Auch Morpheme werden in Gebärdensprachen unterschieden. So gibt es zum Beispiel sogenannte Richtungsverben, welche kongruent mit dem Subjekt sein müssen. In der deutschen Gebärdensprache (DGS) stimmt beispielsweise bei dem Richtungsverb GEBEN der Startpunkt mit dem Subjekt und die Bewegungsrichtung mit dem Empfänger überein. Der Startpunkt und die Bewegungsrichtung sind daher bedeutungstragend (Schwager 2012: 71).

2 Gebärden werden stets in Großbuchstaben angegeben. 
3. Gebärdensprachen sind arbiträr; d.h., es liegt kein direktes Verhältnis zwischen Zeichen und Inhalt vor. Das ist deshalb nicht trivial, weil oftmals für Laien der Eindruck entsteht, Gebärdensprachen seien ikonisch oder bildhaft und daher nicht arbiträr. Tatsächlich sind zwischen ein Drittel und der Hälfte der Gebärden einer Gebärdensprache ikonisch, da anders als in der Lautsprache z.B. Formen dargestellt werden können. Ein solches Zeichen ist AUTO. In etlichen Gebärdensprachen erinnert die entsprechende Gebärde an das Halten des Lenkrads. Dennoch unterscheiden sich an dieser Stelle Gebärdensprache und Lautsprache nicht wesentlich, denn die ikonischen Gebärden können stark variieren. Aus diesem Grund können Menschen, die Gebärdensprachen nicht sprechen, auch durch intensives Zuschauen Äußerungen nicht verstehen bzw. „erraten“ (Boyes Braem 1992: 34ff.). Die DGS ist juristisch seit 2002 als unabhängige Sprache anerkannt (Bundesministerium der Justiz 2002). Aufgrund der genannten Entsprechungen zwischen Laut- und Gebärdensprache wird angenommen, dass sich der Erwerb der beiden Modalitäten ebenfalls gleicht.

\section{Erwerb von Laut- und Gebärdensprachen}

\section{L1-Erwerb gehörloser Kinder mit gehörlosen Eltern}

Da British Sign Language (BSL) und ASL (American Sign Language) besser dokumentiert sind als DGS (Haug 2009: 61) ${ }^{3}$, beziehen sich die Studien zum L1-Gebärdensprachenerwerb meist auf diese beiden. Dennoch können die Ergebnisse der Studien auch auf alle anderen Gebärdensprachen übertragen werden.

Der L1-Gebärdenerwerb wird zunächst durch eine lautliche Lallphase gekennzeichnet, die der Lallphase von hörenden Kleinkindern ähnelt. Ab einem Alter von ca. 9 Monaten beginnen Kinder, manuell zu lallen, d.h., sie produzieren repetitive Gesten. Petitto \& Marentette (1991) beobachteten, dass auch hörende Kinder Anteile von manuellem Lallen aufwiesen und schließen daraus:

The speech modality is not critical in babbling. Rather, babbling is tied to the abstract linguistic structure of language and to an expressive capacity capable of processing different types of signals (signed or spoken). (Petitto \& Marentette 1991: 1493)

3 Bisher liegt lediglich die Studie von Hänel (2005) vor. 
Ab einem Alter von ca. 9 Monaten beginnen gehörlose Kinder, manuelle Gesten zu verwenden, um auf anwesende Personen oder Objekte zu verweisen. Volterra (1986: 11) stellt fest, dass diese Gesten jedoch keine sprachlichen Elemente sind, da sie sich nur auf das Hier und Jetzt beziehen und ausschließlich referentiellen (den konkreten Referenten betreffend) und keinen denotativen (die Kategorie betreffend) Charakter besitzen (vgl. auch Boyes Braem 1992: 164). Ein weiteres Kriterium, das die genannten Gesten von sprachlichen Zeichen bzw. Gebärden unterscheidet, ist ihr Gebrauch in Isolation und ihre Beschränkung auf eine im Kontext verankerte kommunikative Intention. Gebärden hingegen verhalten sich wie sprachliche Ausdrücke und können in verschiedenen Kontexten gebraucht und vor allem mit anderen Elementen kombiniert werden (Volterra 1986: 11). Ab einem Alter von ca. 12 Monaten beginnen gehörlose Kinder, einzelne Gebärden zu benutzen (Boyes Braem 1992: 163f.). Bis zum Alter von 19 Monaten erwerben sie durchschnittlich 60 Gebärden, die jedoch bis zum Alter von etwa drei Jahren in ihrer unflektierten Form gebraucht werden (Haug 2009: 62). Hörende Kinder benutzen ihre ersten Vokalisierungen in konstanter Form ab etwa 0;9 (Jahr; Monat). Diesen frühen Formen wird aber eher eine pragmatische als eine denotative Funktion zugesprochen. Ähnlich wie die erwähnten frühen Gesten gehörloser Kinder handelt es sich hierbei nicht um „symbolisch autonome“ Zeichen, da sie an bestimmte Kontexte, Handlungsabläufe oder Routinen gekoppelt sind und nicht mit anderen Zeichen kombinierbar sind (Kamhi 1986). Die ersten Objektwörter im Sinne der Volterra'schen Definition werden wie die ersten Gebärden gehörloser Kinder etwa bei Vollendung des ersten Lebensjahres geäußert (Bloom 1993: 146ff.). Auch die Phase der Zweiwort-Äußerung im L1-Lautsprachenerwerb entspricht dem Erwerb von Zwei-Gebärdenäußerungen bei gehörlosen Kindern - zu diesem Zeitpunkt werden diese Äußerungen nicht morphologisch markiert (Haug 2009: 62). So ist bspw. noch keine Verbkongruenz zu beobachten (ebd.). Ein weiterer bedeutsamer Faktor in Gebärdensprachen ist der Gesichtsausdruck, der oft in Fragekonstruktionen gebraucht wird. Die erforderlichen Gesichtsausdrücke können bis zu einem Alter von ca. 3;6 Jahren noch nicht zur selben Zeit wie die Gebärden gebraucht werden. Hingegen können Fragen mit Fragewörtern manuell gebärdet werden. Zwischen 2;6 und 3;0 wird 
der Großteil morphologischer Marker erworben (ebd.: 63). ${ }^{4}$ Verbkongruenz wird zwischen 2;0 und 2;6 erworben.

\section{Vergleich zwischen dem Erwerb von Gebärden- und Lautsprachen}

Ein Vergleich zwischen dem Erwerb von Gebärden- und Lautsprachen weist darauf hin, dass jedes Kind ein Stadium des lautlichen Lallens und der Imitation durchläuft, bis es ca. ein Jahr alt ist. Dieses lautliche Lallen jedoch lässt nach einigen Monaten nach, und die Kinder beginnen, manuell zu lallen. Demnach wird der fehlende auditive Input ein entscheidendes Merkmal für den weiteren Spracherwerb und der visuelle Input bekommt größere Bedeutung. Selbst wenn das Lallen in seiner Realisierung verändert wird, hat es dennoch die Funktion, Sprache zu erproben, ungeachtet dessen, ob es gesprochen oder gebärdet ist. Ab einem Alter von 1;6 sind bei gehörlosen und hörenden Kindern Mehrwortäußerungen nachgewiesen (Haug 2009: 62ff.).

Im Jahre 2004 ermittelten Mitchell \& Karchmer (zit. nach Haug 2009: 23), dass wie bereits erwähnt 95\% der gehörlosen Kinder in Deutschland hörende Eltern haben und somit in einem hörenden Umfeld aufwachsen. Diese Kinder erwerben die Gebärdensprache meist erst im Kindergarten oder in der Schule. Aus diesem Grunde ist für diese große Anzahl von Kindern eine verlässliche L1 nicht gesichert, was wiederum einen erheblichen Einfluss auf den L2-Erwerb hat.

\section{Wie kann ein verlässlicher L1-Erwerb für alle gehörlosen Kinder unabhängig vom Hörstatus ihrer Eltern gewährleistet werden?}

Im Folgenden werden die gehörlosen Kinder hörender Eltern in den Blick genommen. Wie dargestellt, ist für diese Kinder i.d.R. kein verlässlicher L1-Erwerb möglich. Vier Ansätze werden vorgestellt, die sich darin unterscheiden, $a b$ wann welche Sprache für die Kinder eingeführt wird. Es

4 Das in der Spracherwerbsforschung angewandte Erwerbskriterium liegt zwischen 80 und $90 \%$ zielsprachlich korrektem Gebrauch eines Phänomens. Das Kriterium wird nicht bei $100 \%$ angesetzt, da selbst erwachsene Sprecher im Erstsprachenerwerb keinen vollständigen Grad der zielsprachlichen Korrektheit erreichen (Ellis 2008: 68). 
wird demnach die Frage gestellt, wie ein angemessener Spracherwerb für alle gehörlosen Kinder ungeachtet des Hörstatus ihrer Eltern gewährleistet werden kann. Diese Überlegungen bilden die Grundlage eines möglichen Englischunterrichts für gehörlose Kinder.

Die sukzessive Methode basiert auf dem Gedanken, dass gehörlose Kinder in die „Mainstream“-Gesellschaft der hörenden Menschen eingegliedert werden sollen. Demnach müssen gehörlose Kinder mit Hilfe der Lautsprache das eigene Sprechen sowie das Lippenablesen lernen (Lynas 2005: 201). Das bedeutet, dass die Lautsprache die erste Sprache darstellt, die gehörlosen Kindern angeboten wird und sie somit die L1 der Kinder ist. Im Gegensatz zur traditionellen oralen Methode wird die Gebärdensprache in diesem Fall nicht vollständig abgelehnt; sie wird jedoch erst angeboten, wenn die gesprochene Sprache erworben ist (Coninx 1994: 61). Das Problem mit diesem Ansatz ist, dass gehörlose Kinder die gesprochene Sprache nicht auditiv wahrnehmen können und auch kein auditives Feedback erhalten können, um ihre eigene Sprache über den auditiven Kanal zu kontrollieren. Das macht es unmöglich für ein gehörloses Kind, die Lautsprache auch nur annähernd altersadäquat im Vergleich zu hörenden Kindern zu erwerben. Darüber hinaus sind die Möglichkeiten des Lippenablesens sehr begrenzt. Jing et al. (2007: 1071) stellen fest, dass 10-30\% an gesprochenen Worten in isolierten Sätzen verstanden werden und ca. 30-60\% der Phoneme in Silben. Laute, die im hinteren Teil des Mundraums gebildet werden, können nur unzureichend identifiziert werden. Das Lippenablesen ist vom visuellen Kanal abhängig, und nicht alle Phoneme haben unterschiedliche Absehgestalten. Der Terminus „Absehgestalt“ oder auch „Kinem“ bezeichnet die verschiedenen Formen des Mundes, die visuell unterschieden werden können, wenn ein Laut produziert wird (ebd.). In der DGS sind 11 Kineme zu verzeichnen, in der deutschen Lautsprache jedoch über 40 Phoneme (je nach Dialekt variierend). Aufgrund dieser Tatsache teilen sich verschiedene Phoneme dieselbe Absehgestalt; sie sind daher kaum auf dem visuellen Kanal voneinander unterscheidbar (vgl. Typolis o.J.). Aus den genannten Gründen ist die sukzessive Methode kein adäquater Spracherwerbs-Ansatz für gehörlose Kinder.

Das schwedische Modell ist ebenfalls eine sukzessive Methode, jedoch ist die Gebärdensprache in diesem Modell die L1 der gehörlosen Kinder. Die 
Lautsprache wird erst ab einem Alter von sechs bis acht Jahren gefördert, und zwar hauptsächlich in Form der geschriebenen Sprache (Coninx 1994: 62f.). Um reichhaltigen Gebärdeninput zu bieten, ist es wesentlich, dass die Eltern die Gebärdensprache erlernen, um sie im Umgang mit den Kindern zu verwenden. Dieses Vorgehen ist jedoch unzureichend, weil Eltern, die erst im Erwachsenenalter die Gebärdensprache lernen, diese nicht, schnell genug' erwerben können, um ihren Kindern einen altersgemäßen Input zu bieten. Das legen Untersuchungen nahe, nach denen Kinder bereits im Alter von sieben Jahren bei einem Test, der morphologische Markierungen in der ASL testete, besser abschnitten als deren Eltern (Haug 2009: 103; Singleton \& Newport 2004). Günther (2012: 34f.) fordert daher ein frühes Interventionsprogramm, durch welches Familien mit gehörlosen Kindern unterstützt werden. Als unerlässlich wird der Kontakt mit einem L1-Sprecher einer Gebärdensprache erachtet, der nicht nur den erforderlichen Input bereitstellt, sondern auch ein gehörloses Vorbild anbietet, mit dem sich gehörlose Kinder identifizieren können. Das schwedische Modell sieht darüber hinaus separaten Gebärdensprachunterricht in der Schule vor, damit gehörlose Kinder auch metasprachliche Kenntnisse erwerben. Wichtig ist, dass neben dem Unterricht in der DGS auch die deutsche Schriftsprache unterrichtet wird, damit die Kinder sowohl in der Gehörlosen- als auch in der Welt/ Community der Hörenden ihren Platz finden.

Der Ansatz der Total Communication (TC) sieht eine simultane Förderung von Gebärden- und Lautsprache von Geburt an vor. Beide Sprachen, die Gebärden- und die Lautsprache, sollen auch gleichzeitig verwendet werden. Das bedeutet, dass sprachliche Information durch zwei Modalitäten ausgedrückt wird, wodurch es zu einer Vermischung beider Sprachen, perzeptuellen Problemen und Verarbeitungsstörungen kommen kann. Es zeigt sich, dass TC nicht zu einer altersadäquaten Beherrschung beider Modalitäten führt - im Gegenteil, die Kinder erwerben keine L1 altersgemäß (Coninx 1994: 64ff.). Auch TC ist daher ungeeignet für gehörlose Kinder.

Das bilinguale Konzept soll sowohl zu einer altersgemäßen L1 führen als auch gehörlose Kinder auf das Leben in beiden Gesellschaften, der gehörlosen und der hörenden, vorbereiten. Ebenso wie TC sollen nach diesem Ansatz die Gebärdensprache sowie die Lautsprache und ihre schriftliche Form gleichzeitig erworben werden. Im Gegensatz zu TC jedoch werden 
niemals beide Modalitäten gleichzeitig bzw. simultan von einer Person benutzt. Beide Sprachen bzw. Modalitäten sind klar voneinander getrennt (Coninx 1994: 63). Um den Ansatz zu realisieren, wird vorgeschlagen, dass Prinzip „Eine Person - eine Sprache“ einzusetzen. In einem Schulkontext bedeutet dies Team Teaching (Günther \& Schäfke 2004: 7). Primär wird im Unterricht die DGS eingesetzt, und zwar als Medium ebenso wie als eigenes Fach. Letzteres soll zu Sprachbewusstheit auf einer metakognitiven Ebene führen, die auch nützlich für die Laut- und Schriftsprache sein soll. Nach den Ergebnissen von Schulversuchen in Berlin und Hamburg hat sich das bilinguale Konzept als erfolgreicher Ansatz zu einer altersgemäßen Entwicklung von Sprache, Kommunikation und Denken gehörloser und hörgeschädigter Kinder erwiesen (Günther \& Schäfke 2004: 23). Über den schulischen Kontext hinaus findet der bilinguale Ansatz auch in der Frühförderung Beachtung (Günther 2012: 10).

Die Intervention soll idealerweise von einem Team aus einem gehörlosen und einem hörenden Pädagogen durchgeführt werden. Auf diese Weise werden dem Kind zwei Rollenmodelle geboten, mit denen es sich identifizieren kann. Auch die hörenden Eltern erhalten einen Einblick in die Gehörlosenwelt (Günther 2012: 34f.).

Das Schwedische Modell und das bilinguale Konzept stellen derzeit die angemessensten Modelle für eine altersgemäße L1-Gebärdensprachentwicklung dar, das gilt sowohl für die Lerner als auch für deren hörende oder gehörlose Eltern. Da die Gebärdensprache eine wesentliche Rolle in der Gehörlosenkultur spielt, kann nur eine Gebärdensprache die L1 gehörloser Kinder sein; keine Lautsprache kann diese Funktion übernehmen (Deuchar \& James 1985: 45). Wichtig ist, dass die Gebärdensprache von Geburt an gefördert wird und dass im Falle hörender Eltern Zugang zu einem Frühförderprogramm besteht, das die Eltern unterstützt, reichhaltigen Gebärdenspracheninput sicherstellt und ein Rollenmodell für die gehörlosen Lerner bietet, indem ein Gebärdenmuttersprachler zur Verfügung steht. Wenn die Kinder in die Schule kommen und mit der Schriftsprache konfrontiert werden, erweist sich das bilinguale Konzept zurzeit als dasjenige, das für die gehörlosen Lerner sowohl den Zugang zur Gehörlosengemeinschaft als auch zur Gemeinschaft der Hörenden bietet. 


\section{Die gegenwärtige Situation des Englischlernens und -lehrens in Deutschland}

Zunächst ist auf den erheblichen Mangel an Forschung bezüglich des Englischlernens als Fremdsprache von gehörlosen Menschen hinzuweisen. Da gehörlose Kinder eine Minderheit darstellen, ist es äußerst schwierig, Investoren zu finden, die die Forschung gehörloser Sprachenlerner finanziell ermöglichen (Dotter 2008: 101-104). Darüber hinaus ist Englisch als Fremdsprache entweder in den Curricula für gehörlose Schülerinnen und Schüler bzw. Studentinnen und Studenten nicht vorgesehen, oder die vorliegenden Curricula werden von Lehrkräften ignoriert, da die gehörlosen Kinder häufig noch mit der Schriftsprache der Mehrheitsgesellschaft Schwierigkeiten haben (ebd.: 101). Da die geschriebenen (und gesprochenen) Varianten der Majoritätssprache bereits die L2 der gehörlosen Lerner sind, haben sie erhebliche Schwierigkeiten, Englisch in welcher Form auch immer zu erwerben (Haug 2009: 24). Die Gebärdensprache und die Majoritätssprache haben unterschiedliche Grammatiken. Der Erwerb der Schriftsprache für gehörlose Kinder stellt daher eine ganz andere Herausforderung als für hörende Kinder dar. Weil gehörlose Kinder die Laute, die die Grapheme der Schriftsprache wiedergeben, nicht wahrnehmen können, werden diese Kinder die Regelmäßigkeiten in der Verbindung zwischen Laut- und Schriftsprache nie erfassen können. Auf diese Weise ist Englisch die dritte Sprache für die Lerner - ein Sachverhalt, der noch komplexer wird, wenn gehörlose Kinder eine Zuwanderungsgeschichte aufweisen und noch eine weitere Sprache berücksichtigt werden muss (Hausen 2013: 101f.). Abgesehen von fehlender Forschung und der Tatsache, dass kein universelles Englisch-Curriculum vorliegt, sind bisher auch keinerlei Lehrkonzepte erprobt worden.

Hinzu kommt die Wahl der Unterrichtssprache. In einer Gebärdensprachklasse ist das Prinzip der Einsprachigkeit mit Hilfe der Lautsprache Englisch offensichtlich nicht angemessen, da wegen der Beeinträchtigung der akustischen Wahrnehmung kaum Informationen aufgenommen werden können. Es müssen also eine weitere Unterrichtssprache oder zumindest lautsprachbegleitende Gebärden eingesetzt werden (ebd.: 103). Die Verwendung einer weiteren Gebärdensprache aus dem englischsprachigen Raum, wie z.B. BSL oder ASL, ist aus zweierlei Gründen problematisch. Zum einen 
bedeutete das neben der Lautsprache Deutsch, DGS, Schriftsprache Englisch eine vierte Sprache. Zum anderen gibt es in Deutschland nicht ausreichend Lehrkräfte, die großflächig ASL oder BSL unterrichten könnten (ebd.: 104f.). Es gäbe noch die Möglichkeit, die Lautsprache Englisch zu benutzen und mit ASL- oder BSL-Gebärden zu begleiten (Signing Exact English, SEE). Dabei werden die einzelnen Gebärden der gewählten Gebärdensprache an die grammatischen Strukturen der Laut- bzw. Schriftsprache angepasst. Darum gilt SEE auch nicht als eine eigenständige Sprache, sondern lediglich als Visualisierungshilfe (ebd.: 105). In der Praxis geschieht es hingegen häufig, dass Lehrkräfte, die in Gebärdenklassen Englisch unterrichten, die englische Lautsprache verwenden, jedoch Gebärden aus der nationalen Gebärdensprache DGS hinzuziehen - oder sie stützen sich gänzlich auf DGS.

Vor allem in lernschwachen Klassen scheint dies den LehrerInnen oft eine geeignete Vorgehensweise zu sein. Sie wollen so das Verstehen der Fremdsprache unterstützen, denn sie befürchten, ihre SchülerInnen durch eine zusätzliche Sprache zu überfordern. Bei einem solchen Vorgehen steht meist der Erwerb der englischen Schriftsprache im Vordergrund, und die Gebärdensprache wird eingesetzt, um deren Struktur, Grammatik und Vokabeln zu erklären. Kritisch anzumerken ist an diesem zunächst sehr einleuchtenden Verfahren, dass die Verknüpfung der nationalen Gebärden mit der Fremdsprache Englisch keine authentische Sprachkombination darstellt [...]. (ebd.: 106)

\section{Die Bedeutung für gehörlose Kinder, Englisch zu lernen}

Viele Menschen sind überrascht, wenn sie hören, dass gehörlose Kinder Englisch lernen, wenn doch bereits die nationale Laut- und Schriftsprache solch eine enorme Herausforderung darstellen. Warum müssen also noch weitere Sprachen hinzukommen? Zuerst einmal stellen in Deutschland gute Englischkenntnisse eine Voraussetzung dar, um einen Schulabschluss zu erlangen. Dies gilt auch für gehörlose Schülerinnen und Schüler. Dementsprechend ist der Englischunterricht für gehörlose Schülerinnen und Schüler unumgänglich, um ihnen die gleichen Bildungschancen zu bieten wie hörenden Kindern. Sie hätten ansonsten nicht dieselben beruflichen Chancen, die hörenden Lernern gegeben werden. Auch bei der Stellensuche sind Englischkompetenzen eine Schlüsselqualifikation, weil sie den Umgang mit der modernen, durch das Englische geprägten Informationstechnologie und dem Internet ermöglichen. Englischkenntnisse stellen zudem ein bedeutsames Werkzeug zum eigenständigen Wissenserwerb dar (ebd.: 99; 
Soerensen 2002: 173). Auch wenn die grundsätzliche Sinnhaftigkeit und Notwendigkeit des Englischunterrichts klar ist, ist es wichtig, einen Blick auf die spezifische Nützlichkeit und Umsetzbarkeit der unterschiedlichen Zielsprachen zu werfen. Ist es für gehörlose Schülerinnen und Schüler nützlich, die englische Lautsprache oder die englische Schriftsprache zu lernen? Oder ist es sinnvoll für gehörlose Schülerinnen und Schüler, eine weitere Gebärdensprache, z.B. ASL oder BSL, zu erwerben?

\section{Das Unterrichten der englischen Lautsprache}

Gehörlose Kinder können, wie dargestellt, keine Sprache über den auditiven/ oralen Kanal erwerben, und sie können auch kein auditives Feedback erhalten. Auch das Lippenablesen kann dieses Problem nicht lösen - mit dem Effekt, dass gehörlose Kinder keine Erfolgserlebnisse im Erwerb der Lautsprache haben. Hinzu kommt, dass der Lautspracherwerb nicht altersgemäß ist in Bezug auf die grammatischen und kommunikativen Fähigkeiten hörender Kinder (Haug 2009: 25). Gesprochenes Englisch hat daher eine sehr geringe Relevanz für das Leben gehörloser Kinder (Fleming 2008: 123). Kinder, die mit einem Cochlea-Implantat ${ }^{5}$ technisch versorgt sind, sind hier ausgenommen, da sie lautsprachlich erzogen werden und angenommen wird, dass sie sich in die Gemeinschaft der Hörenden integrieren können.

\section{Das Unterrichten von geschriebenem Englisch}

Gehörlosen Kindern die englische Schriftsprache zu vermitteln, erscheint wesentlich. Die deutsche Laut- und Schriftsprache ist stark von Anglizismen geprägt, und die Jugendkultur ist von der englischen Sprache beeinflusst. Insbesondere erfordert der Gebrauch des Internets Kompetenzen in der englischen Schriftsprache. Im Jahre 2002 enthielten 72\% der damals drei Millionen öffentlichen Webseiten englischsprachige Inhalte (Fleming 2008: 128; Hausen 2013: 100). Darüber hinaus ist ASL erheblich besser dokumentiert und erforscht als DGS. Diese Forschung ist jedoch fast ausschließlich

5 Cochlea-Implantate $(\mathrm{CI})$ sind chirurgisch eingesetzte elektronische Geräte, die bei den Patienten sinnvoll sind, deren Hörschädigung im Innenohr vorliegt bzw. dort, wo die inneren Haarzellen nicht einwandfrei funktionieren. Auditive Neuronen werden mit Hilfe von implantierten Elektroden elektrisch stimuliert (Svirsky 2011: 215). 
in englischer Sprache erhältlich. Somit stellt die englische Schriftsprache ein wichtiges Medium dar, um einerseits Weltwissen und andererseits neue Erkenntnisse über Gebärdensprachen zu erlangen (Haug 2009: 61). Dabei muss jedoch auch beachtet werden, dass gehörlose Kinder oft große Probleme mit der deutschen Schriftsprache haben, da sie nicht DGS repräsentiert, sondern die deutsche Lautsprache. Es ist deshalb zu erwarten, dass der Erwerb der englischen Schriftsprache eine ebenso große Herausforderung darstellt (Dotter 2008: 100). Zudem bietet die Schriftsprache kein angemessenes Medium für die direkte Kommunikation. „Written communication often differs from spoken communication in terms of formality, grammar, completeness of utterances and the pattern of interaction" (ebd.). Die Interaktionsmuster der Schriftsprache sind nicht ökonomisch genug für eine Kommunikation von Angesicht zu Angesicht.

\section{Das Unterrichten einer weiteren Gebärdensprache}

Eine weitere Gebärdensprache könnte als Kommunikationsmedium vermittelt werden, um die Grenzen der englischen Schriftsprache zu kompensieren. Das würde bedeuten, dass die Gehörlosen-Gemeinschaft sich auf eine Sprache einigen müsste, die die Funktionen und den Status der englischen Lautsprache übernehmen kann. Dotter (2008: 100) erinnert daran, dass Englisch die lingua franca der Europäischen Gemeinschaft ist und vielleicht das wichtigste Kommunikationsmittel für weltweite Kommunikation. Welche Gebärdensprache könnte jedoch die Funktionen der englischen Lautsprache übernehmen? Um diese Frage zu beantworten, ist zu beachten, dass es zwar Internationale Gebärden gibt, welche in Form einer Pidgin-Sprache als lingua franca der Gebärdensprache gelten könnten, diese jedoch in der Tiefe der Kommunikation und in ihrer Ausdrucksfähigkeit, im Vergleich zu einer richtigen Gebärdensprache, begrenzt sind (vgl. European Union of the Deaf EUD 2011). Somit können die Internationalen Gebärden nur einen Teil der englischen Lautsprache übernehmen und kompensieren. Darüber hinaus muss auf der Suche nach einer geeigneten Gebärdensprache die Tatsache beachtet werden, dass es nicht die eine englische Gebärdensprache gibt, sondern viele verschiedene, die in der englischsprachigen Welt gebraucht werden. Die bekanntesten dieser Gebärdensprachen sind ASL und BSL. Die Entscheidung für die eine oder die andere ist nicht trivial, da beide sich sehr stark in der Grammatik, den Gebärden und sogar dem 
Fingeralphabet unterscheiden (Hausen 2013: 105). Für ASL spricht die Tatsache, dass die USA als Hochburg der Gebärdensprachforschung angesehen werden kann. Die Gallaudet University (Washington D.C.) für Schwerhörige und Gehörlose lockt zahlreiche internationale Studierende an. Zudem ist ASL Verkehrssprache auf internationalen Gehörlosen-Kongressen und wird auch verstärkt in internationalen Privatkonversationen verwendet. Es gibt zahlreiche Unterrichtsmaterialien wie z.B. ASL-Wörterbücher, Englischbücher in ASL und Videos von Muttersprachlern (Poppendieker 2011: 24; Hausen 2013: 105). ASL wird bereits in den skandinavischen Ländern als lingua franca genutzt (Günther \& Schäfke 2004: 46). Für BSL hingegen spricht die räumliche Nähe zu Großbritannien, so dass leichter Schüleraustausche organisiert werden können, um BSL-Kommunikationssituationen für die Schülerinnen und Schüler aber auch die Lehrpersonen zu ermöglichen. Diese Projekte können finanziell von der EU gefördert werden. Außerdem hat auch BSL internationale Relevanz, da die Gebärdensprachen aus Teilen Afrikas, Asien und Australien BSL hinreichend ähnlich sind. Dadurch findet auch BSL in internationalen privaten Konversationen Anwendung (Pritchard 2011: 15; Hausen 2013: 105).

Es wird deutlich, dass es sowohl für ASL, als auch für BSL gute Argumente gibt, die englische Lautsprache in ihrer kommunikativen Funktion und ihrem internationalen Status für die Gehörlosenwelt ersetzen zu können. Egal welche der beiden Sprachen für den Englischunterricht ausgewählt wird, es wäre bedeutsam, dass man sich möglichst bundesweit auf eine Gebärdensprache mit internationalem Status einigt, so dass auch ein Austausch von Materialien und gemeinsame Konzepte möglich sind (ebd.).

\section{Die schwierige Wahl der Unterrichts- und Zielsprache im Englischklassenraum - Möglichkeiten und Grenzen}

Die Diskussion hat gezeigt, dass sich die englische Lautsprache für gehörlose Kinder nicht eignet, aber dass das Lernen der englischen Schriftsprache sowie einer weiteren Gebärdensprache wie ASL oder BSL sinnvoll ist. Die zusätzliche Gebärdensprache kann den Status und die Funktion der gesprochenen Sprache übernehmen und so als Pendant zur englischen Schriftsprache gesehen werden. Folgende Möglichkeiten ergeben sich für den Einsatz weiterer Sprachen nach der obigen Diskussion: 
1. Es wird nur die Schriftsprache oder nur eine weitere Gebärdensprache unterrichtet.

2. Es wird zunächst eine weitere Gebärdensprache unterrichtet und die englische Schriftsprache danach folgend.

3. ASL/BSL und die englische Schriftsprache werden gleichzeitig unterrichtet.

Es wird nur die Schriftsprache oder nur eine weitere Gebärdensprache unterrichtet

Abb. 1: Alternative 1

Deutsche Gebärdensprache

Deutsche Laut- und Schriftsprache

$$
\downarrow
$$

Eine weitere Gebärdensprache (ASL/BSL)

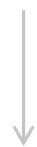

Englische Schriftsprache

Wenn man sich entschließt, eine weitere Gebärdensprache wie ASL oder BSL (dunkler Pfeil) zu unterrichten bzw. nur das geschriebene Englisch (heller Pfeil), würde man einen bedeutenden Teil der Sprache vernachlässigen. Wie in 6.2 ausgeführt, hat das geschriebene Englisch eine große Bedeutung für gehörlose Menschen im Hinblick auf die Informationstechnologie und den Aufbau von Weltwissen. Würde nur eine zusätzliche Gebärdensprache hinzukommen, könnte nicht garantiert werden, dass die Kinder die genannten Vorteile der englischen Schriftsprache nutzen können. Dieser Ansatz würde auch bedeuten, dass die Anforderungen für einen deutschen Schulabschluss geändert werden müssten. Der alleinige Einsatz der Schriftsprache würde die kommunikativen Funktionen der Sprache vernachlässigen, da sie eben in sozialen und interaktiven Kontexten keine Rolle spielt (Svartholm 1994: 64). Die Kinder würden demnach kein Kommunikationsmittel erwerben, mit dem sie weltweit kommunizieren könnten. 
Es wird zunächst eine weitere Gebärdensprache (ASL/BSL) unterrichtet und die englische Schriftsprache danach folgend

\section{Abb. 2: Alternative 2}

Deutsche Gebärdensprache

Deutsche Laut- und Schriftsprache

Eine weitere Gebärdensprache (ASL/BSL)

Englische Schriftsprache

Der Ansatz, zunächst eine weitere Gebärdensprache und dann die englische Schriftsprache zu unterrichten, resultiert aus der folgenden Überlegung: Je älter ein gehörloses Kind ist, desto besser kann es die Schriftsprache erwerben (Svartholm 1994: 66). Üblicherweise wird davon ausgegangen, dass ein Kind umso besser eine neue Sprache lernen kann, je jünger es ist. Diese Annahme trifft jedoch auf sekundäre, nicht-natürliche Sprachen wie die Schriftsprache nicht zu. Sie wird in einem formalen Unterrichtskontext gelehrt und erfordert eine gewisse kognitive Reife, die von sehr jungen Kindern nicht erwartet werden kann (ebd.: 65). Eine weitere Gebärdensprache kann jedoch in einer kommunikativen, lebhaften und „natürlicheren“ Weise unterrichtet werden (Hausen 2013: 105), vergleichbar mit dem Englischunterricht der Grundschule für hörende Kinder. Im Englischunterricht der Grundschule basieren die Stunden auf Themen und kommunikativen Situationen, die den Interessen und der Lebenswelt der Kinder entsprechen. Die Progression der Kinder ist daher vorwiegend kommunikativ, themenorientiert und natürlich (Roos 2006: 24ff.). Die Annahme, dass eine Gebärdensprache umso besser erlernt wird, je jünger das Kind ist, scheint nach den bisherigen Erfahrungen zuzutreffen (Haug 2009). Daher scheint es angemessen, zuerst eine zweite Gebärdensprache einzuführen und dann die englische Schriftsprache. 
ASL/BSL und die englische Schriftsprache werden gleichzeitig unterrichtet Abb. 3: Alternative 3

Deutsche Gebärdensprache Deutsche Laut- und Schriftsprache
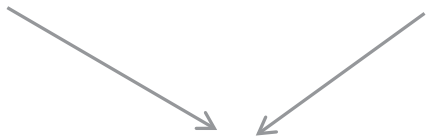

Eine weitere Gebärdensprache (ASL/BSL)

Englische Schriftsprache

Das bilinguale Konzept bezieht auch die englische Schriftsprache und die jeweils ausgewählte Gebärdensprache ASL oder BSL ein. Kernstück des bilingualen Konzepts, so wie es innerhalb des Hamburger BilingualismusModells vertreten wird, ist der im Team von hörender und gehörloser Lehrkraft gemeinsam durchgeführte bilinguale Unterricht in Gebärden-, Schrift- und Lautsprache und damit - unabhängig vom erreichten Entwicklungsniveau in den einzelnen Sprachmodalitäten - auch der Einsatz der Lautsprache in der unterrichtlichen Kommunikation von Anfang an (Günther \& Schäfke 2004: 7). Die folgenden Prinzipien und Ziele liegen dem Konzept zugrunde: 
Tab. 1: Prinzipien und Ziele des bilingualen Konzepts (Günther \& Schäfke 2004: 7)

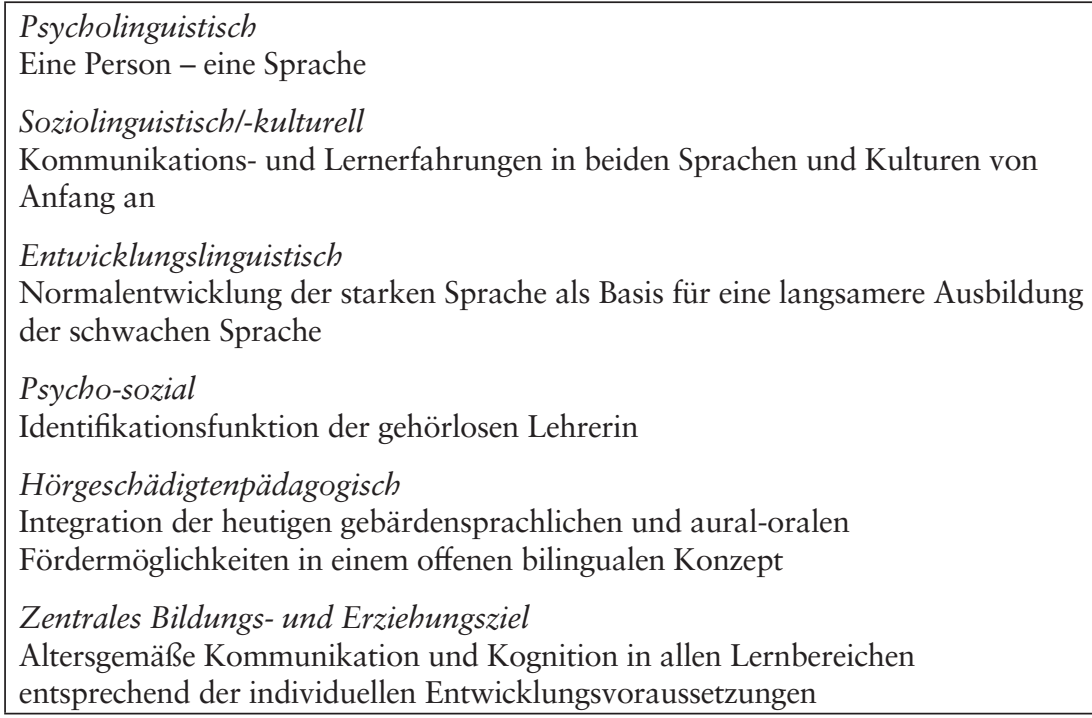

Die nicht gebärdende Lehrkraft benutzt das Schrift-Englisch ebenso wie gesprochenes Englisch. Zusätzlich wird SEE (Signing Exact English, s.o.) eingesetzt, das die Laut- und Schriftsprache begleitet und verdeutlicht. Da SEE sich an den grammatischen Strukturen des geschriebenen und gesprochenen Englisch orientiert, wird es nicht als separate Sprache angesehen (Hausen 2013: 105). Die Gebärden- und Schriftsprache werden demnach gemeinsam sowohl als Unterrichtsmedium als auch als Unterrichtsgegenstand eingesetzt.

Sowohl der bilinguale Ansatz als auch das sukzessive Unterrichten von zunächst einer weiteren Gebärdensprache (ASL bzw. BSL) und anschließend der englischen Schriftsprache können gehörlose Kinder angemessen auf ein Leben in „einer absolut übermächtig laut- und schriftsprachlich dominierten Welt“ (Günther \& Schäfke 2004: 11) vorbereiten. Beide Ansätze ermöglichen den Zugang zu englischsprachigen akademischen Texten und zu einem Arbeitsleben, in dem Englischkenntnisse unerlässlich sind. Darüber hinaus wird durch das Erlernen einer Gebärdensprache mit internationalem Status den gehörlosen Kindern ermöglicht, internationale Privatgespräche zu führen und an internationalen Kongressen der Gehörlosenwelt teilzunehmen. Dennoch entsteht ein Problem, wenn versucht wird, die an gehörlose Kinder gestell- 
ten Kompetenzerwartungen mit denen hörender Kinder zu vergleichen. Seit dem Schuljahr 2008/09 wird in Nordrhein-Westfalen Englisch vom zweiten Halbjahr des 1. Schuljahres an (beginnend im Februar) unterrichtet (Schulministerium 2008). Die Einführung einer Fremdsprache geschieht demnach dreieinhalb Jahre früher, verglichen mit der Zeit vor 2003, als Englisch zu Beginn des 5. Schuljahres als neues Schulfach hinzukam. Wenn gehörlose Kinder bereits im 1. Schuljahr Englisch lernen sollten, würde das bedeuten, dass sie entweder zwei Fremdsprachen, ASL/BSL und die deutsche Schriftsprache, oder nach dem bilingualen Konzept sogar drei Fremdsprachen (ASL/ BSL, die deutsche und die englische Schriftsprache) lernen müssten. Hinzu kommt, dass bei Zuwanderungsgeschichte eine weitere L1 mit Hilfe eines frühen Interventionsprogramms gesichert werden müsste (Svartholm 1994: 66). Aus diesem Grunde scheint es nur vernünftig zu sein, Englisch für gehörlose Kinder ab dem 5. Schuljahr einzusetzen. Trotz eines späteren Beginns könnte befürchtet werden, dass der gleichzeitige Kontakt zu ASL/BSL und der englischen Schriftsprache die Kinder überfordert (Günther \& Schäfke 2004: 7). Das würde bedeuten, dass ASL/BSL z.B. ab dem 5. Schuljahr unterrichtet und die englische Schriftsprache von Klasse 6 an eingeführt würde. Alternativ würden nach dem bilingualen Konzept ASL/BSL und die Schriftsprache zusammen im 5. Schuljahr eingesetzt werden. Das würde in jedem Falle bedeuten, dass gehörlose Kinder später Englisch lernen als hörende und die Kompetenzerwartungen nicht dieselben sein könnten, sondern angepasst werden müssten. Andererseits wären die Bildungschancen und späteren Berufsaussichten für hörende und gehörlose Jugendliche nicht dieselben.

\section{Methodische Überlegungen}

Nachdem die Bedingungen und Voraussetzungen für den Englischunterricht gehörloser Lerner diskutiert worden sind, stellen sich methodische Fragen. Wie können ASL/BSL und daran anschließend die englische Schriftsprache effektiv unterrichtet werden?

Das Unterrichten einer weiteren Gebärdensprache kann kommunikativ erfolgen, und zwar dadurch, dass möglichst lebensnahe Situationen und Kontexte geschaffen werden, in denen die Lerner mit Hilfe visueller Hilfsmittel bereits ahnen oder wissen, worum es thematisch geht (z.B. beim Storytelling mit Hilfe von Bilderbüchern, vgl. Hausen 2013: 110). Die neue 
Gebärdensprache begleitet daher zu Beginn die Kontexte so, wie es die Lautsprache auch tut. Die Situation ist daher durchaus mit dem frühen Englischunterricht der Grundschule zu vergleichen, in der die Sprache als Handlungsinstrument erfahren wird. Das kann nur gelingen, wenn die Sprache auch von Beginn an handlungsorientiert eingesetzt wird (Bach \& Timm 2013) und auf expliziten Grammatikunterricht verzichtet wird (Roos 2006). Ein zentrales Ziel von handlungsorientiertem Unterricht muss sein, ein kommunikatives Bedürfnis zu schaffen (create a need, Schäfer 2013: 48). Handlungsorientiertes Unterrichten kommt hingegen für das Unterrichten der Schriftsprache nur in geringerem Maße (bspw. für das kreative Schreiben) in Frage, da sie (vorwiegend) für die Kommunikation, die von Ort und Zeit unabhängig ist, entwickelt wurde (Svartholm 1994: 65).

Für das Erlernen der englischen Schriftsprache liegen Erfahrungen von der Universität Wolverhampton vor. Dort wurde 1998 ein Englischkurs eingerichtet, in dem hörgeschädigte bzw. gehörlose internationale Studierende Englisch lernen. Es wurden dort BSL, SEE und das Fingeralphabet als Metasprache eingesetzt, mit dem Ziel, die englische Schriftsprache und ihre Grammatik zu vermitteln. Die Gebärdensprache wurde eingesetzt, um neue bisher unbekannte grammatische Strukturen zu diskutieren. Voraussetzung für diesen Kurs waren gute Kenntnisse des BSL (Fleming 2008; Hausen 2013: 107). Auch in diesem Englischkurs war es bedeutsam, dass Grammatik und Vokabular in einem für die Lernenden relevanten Kontext angeboten werden dieses Prinzip gilt für Kinder ebenso wie für junge Erwachsene (Fleming 2008: 144). Visuelle Unterstützung ist für gehörlose Kinder besonders wichtig, da sie nicht über ein auditives Gedächtnis verfügen. Fleming arbeitete daher mit Handouts, auf denen Schlüsselwörter, Abbildungen oder kurze Texte zu finden waren, um den Lernprozess zu unterstützen (ebd.: 137).

Weiterhin wird angenommen, dass der Einsatz verschiedener Prinzipien der Sicherung von Gelerntem dienen kann. Von den sieben Prinzipien, die Nunan (2004) für das Task-Based Teaching and Learning anführt, erscheinen besonders das Scaffolding und das Recycling als bedeutsam. Beim Scaffolding wird der Lerner durch häufig wiederkehrende Sprachmuster unterstützt (Gibbons 2009). ${ }^{6}$ Das Prinzip des Recycling impliziert, dass die

6 „A scaffold can be described as situated help for the students, for example making use of holistic linguistic structures (formulaic sequences or chunks), visual materials 
Lerner erfahren, wie die zielsprachlichen Items sowohl in eng verwandten als in völlig unterschiedlichen Kontexten gebraucht werden. Entscheidend ist hierbei, dass ein sprachliches Item in bestimmten Abständen immer wieder thematisiert wird (Nunan 2004: 36f.).

Zur weiteren Unterstützung können Online English Language TeachingWebsites eingesetzt werden. Sie können ein effektives Lernen der englischen Schriftsprache vorantreiben, indem besonders grammatische Formen und Vokabeln abgefragt und gesichert werden (Fleming 2008: 144).

In Bezug auf das Vokabellernen erscheint es auch sinnvoll zu sein, die englische geschriebene Form mit der entsprechenden ASL- oder BSLGebärde und einem Bild zu verknüpfen. Einige Vokabeln jedoch können nur schwer dargestellt werden, so wie „yes“ oder „what“. In diesen Fällen ist eine Verknüpfung mit der deutschen geschriebenen Form unumgänglich.

\section{Fazit}

Da Gebärdensprachen und Lautsprachen natürliche Sprachen sind, sind die Erwerbsprozesse beider Modalitäten vergleichbar und weisen universelle Züge auf. Bedeutsam ist hierbei, dass diese Vergleichbarkeit nur dann vorliegt, wenn der Erwerb der Gebärdensprache von gehörlosen Kindern mit gehörlosen Eltern betrachtet wird und die Lautsprache entsprechend von hörenden Kindern mit hörenden Eltern. Beide Gruppen durchschreiten vergleichbare Stadien bei gleichem Alter.

Die Situation ist eine andere für gehörlose Kinder mit hörenden Eltern, eine Konstellation, die auf 95\% der gehörlosen Kinder zutrifft (Haug 2009: 23). Oftmals ist ihre L1 DGS verzögert, da sie erst Zugang zur Gebärdensprache bei Eintritt in Kindergarten oder Schule erhalten. Es wurde gezeigt, dass das Schwedische Modell mit Hilfe eines frühen Interventionsprogramms sowie das bilinguale Konzept allen gehörlosen Lernern einen angemessenen L1-Erwerb der Gebärdensprache ermöglichen können - unabhängig vom Hörstatus der Eltern.

Der altersgemäße L1-Erwerb hat einen positiven Einfluss auf den Erwerb weiterer Sprachen, was wiederum für die gehörlosen Lerner äußerst

or signs and symbols for activities, the major idea being that 'with assistance, learners can reach beyond what they can do unaided'“ (Gibbons 2009: 15). 
bedeutsam ist, denn die schriftliche Variante der Majoritätssprache ist bereits ihre L2. Weiterhin ist der altersgemäße L1-Erwerb für den Erwerb weiterer Fremdsprachen, wie ASL oder BSL und der englischen Schriftsprache, entscheidend. Hierbei muss beachtet werden, dass es sich bei ASL und BSL, anders als bei den Lautvarianten des British und American English, um völlig verschiedene Gebärdensprachen handelt, so dass es unerlässlich wäre, sich bundesweit für eine der beiden Sprachen zu entscheiden. Obwohl Großbritannien Deutschland geographisch näher ist, sprechen zahlreiche Faktoren für ASL, da es hierfür bereits erprobte Lehrmaterialien gibt und ASL die in internationalen Kontexten am häufigsten eingesetzte lingua franca für die Gehörlosen-Community darstellt.

Für das Unterrichten von ASL/BSL eignen sich parallel zum Lautsprachenunterricht handlungsorientierte methodische Ansätze (Storytelling, Task-Based Learning), da die Lerner in diesen Fällen die Sprache als Handlungsinstrument erfahren und sie am ehesten in die Lage versetzt werden, das neu erlernte Medium in bedeutsamen Kontexten selbst benutzen zu müssen. Für den Erwerb der Schriftsprache liegen bisher nur dokumentierte Erfahrungen für hörgeschädigte und gehörlose Universitätsstudierende vor (Fleming 2008). Aber auch diese Erfahrungen lassen sich wie gezeigt auf jüngere gehörlose Lerner übertragen.

Besonders erwähnenswert ist im Hinblick auf Inklusion das Problem, zu welchem Zeitpunkt Englisch in Form einer Gebärdensprache und ggf. als Schriftsprache eingeführt wird. Es ist aufgrund des Gesagten nicht ratsam, bereits in der Grundschule mit ASL/BSL und der Schriftsprache Englisch zu beginnen. Unabhängig davon, wie man sich entscheidet, die Sprachen entweder gleichzeitig ab Klasse 5 oder ab diesem Zeitpunkt sukzessive einzuführen, müssen die Kompetenzerwartungen in Bezug auf Englisch für gehörlose Lerner angepasst werden und sind daher keinesfalls mit den Erwartungen an hörende Kinder kompatibel. Außerdem besteht das Problem, dass die sukzessive Einführung in inklusiven Settings bedeuten würde, die gehörlosen Kinder auszuschließen, wenn für die anderen bereits ab der ersten Klasse mit Englisch begonnen wird und dementsprechend überall im Klassenraum englische Schriftsprache präsent sein wird.

Zwei Aspekte müssen abschließend erwähnt werden. Zum einen wird es aufgrund der geringen Anzahl von gehörlosen Lernern keine Lerngruppen geben, die sich tatsächlich nur aus diesen Lernern konstituieren. Das 
bedeutet, dass die Klassen in dieser Hinsicht immer heterogen bleiben werden, da sie aus gehörlosen, zu verschiedenen Graden hörgeschädigten sowie eher lautsprachlich- oder eher gebärdenorientierten Lernern bestehen werden. Eine „interne Inklusion“, die auf der Basis des bilingualen Konzepts all diese Lerner einschließt, ist denkbar. Eine Lehrkraft würde gesprochenes Englisch benutzen und würde es für die gehörlosen Lerner mit SEE unterstützen und weiterhin die Schriftsprache Englisch einsetzen. Die zweite Lehrkraft würde dann ASL oder BSL benutzen. Diese Möglichkeit jedoch ist derzeit nur eine Wunschvorstellung, da es nur wenige Lehrkräfte in Deutschland gibt, die die entsprechenden Fähigkeiten in ASL oder BSL besitzen. Zu einem gewissen Grad wäre es denkbar, dieses Defizit durch Videoaufnahmen zu kompensieren. Auf diese Weise allein aber kann keine Sprache erlernt werden, da keine wirkliche Interaktion stattfinden kann.

Wünschenswert wäre darüber hinaus, dass der Förderschwerpunkt Hören an den Universitäten enger mit den Englisch-Instituten zusammenarbeitet, da es wichtige Überschneidungen in den methodisch-didaktischen Ansätzen gibt. Selbst wenn noch etliche Fragen in Bezug auf den Englischunterricht für gehörlose Lerner bleiben, so lässt sich doch Folgendes konstatieren: „more progress has been made in educating deaf students during the last 30 years than in the previous 300" (Marshark et al. 2002: x).

\section{Literaturverzeichnis}

Bach, Gerhard \& Timm, Johannes-Peter (2013), Handlungsorientierung als Ziel und als Methode. In: Bach, Gerhard \& Timm, Johannes-Peter (Hrsg.) (2013), Englischunterricht (5. Aufl.). Tübingen et al.: Francke, 1-22.

Bartosch, Roman \& Rohde, Andreas (Hrsg.) (2013), Im Dialog der Disziplinen. Englischdidaktik - Förderpädagogik - Inklusion. Trier: Wissenschaftlicher Verlag Trier.

Battison, Robbin (1978), Lexical Borrowing in American Sign Language. Silver Spring, MD: Linstok.

Becker, Claudia \& von Meyenn, Alexander (2012), Phonologie: Der Aufbau Gebärdensprachlicher Zeichen. In: Eichmann, Hanna; Hansen, Martje \& Heßmann, Jens (Hrsg.) (2012), 31-57.

Bloom, Lois (1993), The Transition from Infancy to Language. Acquiring the Power of Expression. Cambridge: Cambridge University Press. 
Boyes Braem, Penny (1992), Einführung in die Gebärdensprache und ihre Erforschung. Hamburg: Signum Verlag.

Brown, Keith (Hrsg.) (2006), Encyclopedia of Language and Linguistics. Elsevier.

Bundesministerium der Justiz (2002) [Online: http://www.gesetzte-iminternet.de/bgg/BJNR146800002.html. 10.04.2015].

Coleman, John S. (2006), Design features of language. In: Brown, Keith (Hrsg.) (2006), 471-474.

Coninx, Frans (1994), Erziehung hörgeschädigter Kinder zur Lautsprache warum, wann und wie? In: Berufsverband Deutscher Hörgeschädigtenpädagogen (Hrsg.) (1994), Von der Taubstummenbildung zur Hörgeschädigten Pädagogik -Erziehung zur Sprache im Wandel. Leipzig: $\mathrm{BDH}$, 52-71.

Deuchar, Margaret \& James, Helen (1985), English as the second language of the deaf. Language \& Communication 5: 1, 45-51.

Dotter, Franz (2008), English for Deaf Sign Languages Users: Still a Challenge. In: Kellett Bidoli, Cynthia J. \& Ochse, Elana (Hrsg.) (2008), 87-121.

Eichmann, Hanna; Hansen, Martje \& Heßmann, Jens (Hrsg.) (2012), Handbuch Deutscher Gebärdensprache-Sprachwissenschaftliche und anwendungsbezogene Perspektiven. Seedorf: Signum, Band 50.

Ellis, Rod (2008), The Study of Second Language Acquisition (2. Aufl.). Oxford: Oxford University Press.

European Union of the Deaf EUD (2011), [Online: http://www.eud.eu/ International Sign_Disclaimer-i-206.html 10.04.2015].

Fleming, Joan (2008), How should we teach deaf learners? Teaching English as a written language to deaf European students. In: Kellett Bidoli, Cynthia J. \& Ochse, Elana (Hrsg.) (2008), 123-153.

Gibbons, Pauline (2009), English Learners, Academic Literacy, and Thinking: Learning in the Challenge Zone. Portsmouth, NH: Heinemann.

Günther, Klaus-B. (2012), Bilingual aufwachsen - Gebärdensprache in der Frühförderung hörbehinderter Kinder. [Online: http://www.gehoerlosenbund.de/dgb/images/stories/pdfs_presse/dgb_brosch_fruehfoerdeung_2012_web_pdf 10.04.2015].

Günther, Klaus-B. \& Schäfke, Ilka (2004), Bilinguale Erziehung als Förderkonzept für gehörlose SchülerInnen- Abschlussbericht zum Hamburger Bilingualen Schulversuch. Hamburg: Signum Verlag. 
Hänel, Barbara (2005), Der Erwerb der Deutschen Gebärdensprache als Erstsprache. Die frühkindliche Sprachentwicklung von Subjekt-und Objektverbkongruenz in DGS. Tübingen: G. Narr.

Haug, Tobias (2009), Adaptation and Evaluation of a German Sign Language Test. Hamburg: Hamburg University Press.

Hausen, Amelie (2013), Englisch an der Schule für Hörgeschädigte - Chance oder Überforderung? In: Bartosch, Roman \& Rohde, Andreas (Hrsg.) (2013), 95-114.

Kamhi, Alan G. (1986), The elusive first word: The importance of the naming insight for the development of referential speech. Journal of Child Language 13, 155-161.

Kellett Bidoli, Cynthia J. \& Ochse, Elana (Hrsg.) (2008), English in International Deaf Communication. Bern: International Academic Publishers, Volume 72.

Leonhardt, Annette (2002), Einführung in die Hörgeschädigtenpädagogik (2. Aufl.). München \& Basel: Ernst Reinhardt Verlag (UTB 2002).

Lynas, Wendy (2005), Controversies in the education of deaf children. Current Paediatrics 15, 200-206.

Marshark, Marc; Lang, Harry G. \& Albertini, John A. (2002), Educating Deaf Students: From Research to Practice. New York: Oxford University Press.

Nunan, David (2004), Task-Based Language Teaching. Cambridge: Cambridge University Press.

Petitto, Laura Ann \& Marentette, Paula F. (1991), Babbling in the manual mode: Evidence for the ontogeny of language. Science 251: 5000, 1493-1496.

Poppendieker, Renate (2011), Englischunterricht im bilingualen Konzept. Diskussionsrunde: Bilingualer Englischunterricht mit ASL, BSL oder DGS. DGFS-Forum 19, 24-28.

Pritchard, Patricia (2011), Using a sign language in teaching of English to deaf pupils. DFGS-Forum 19, 12-23.

Roos, Jana (2006), Frühes Fremdsprachenlernen: Eine Standortbestimmung. In: Pienemann, Manfred; Keßler, Jörg-Uwe. \& Roos, Eckhard (Hrsg.) (2006), Englischerwerb in der Grundschule. Paderborn: Verlag Ferdinand Schöningh, 24-32. 
Sandler, Wendy (2006), Sign Language: Overview. In: Brown, Keith (Hrsg.) (2006), 328-338.

Schäfer, Ulla (2013), Englischunterricht für Schülerinnen und Schüler mit Lernschwierigkeiten. In: Bartosch, Roman \& Rohde, Andreas (Hrsg.) (2013), 45-62.

Schulministerium NRW (2008), Lehrplan Englisch Grundschule. Ministerium für Schule und Weiterbildung des Landes Nordrhein Westfalen. Richtlinien und Lehrpläne für die Grundschule in Nordrhein Westfalen. Frechen: Ritterbach Verlag GmbH.

Schwager, W. (2012), Morphologie: Bildung und Modifikation von Gebärden. In: Eichmann, Hanna; Hansen, Martje \& Heßmann, Jens (Hrsg.) (2012), 61-111.

Siebert-Ott, Gesa (2001), Frühe Mehrsprachigkeit. Tübingen: G. Narr.

Singleton, Jenny L. \& Newport, Elissa L. (2004), When learners surpass their models: The acquisition of American Sign Language from inconsistent input. Cognitive Psychology 49, 370-407.

Soerensen, Noelle (2002), "Englisch - auch das noch?" oder "Englisch eine neue Herausforderung?!” Hörgeschädigtenpädagogik 4, 172-179.

Stokoe, William (1960), Sign language structure: An outline of the visual communication systems of the American deaf. Studies in Linguistics: Occasional Papers (No. 8). Dept. of Anthropology and Linguistics; University of Buffalo.

Svartholm, Kristina (1994), Second language learning in the deaf. In: Ahlgren, Inger \& Hyltenstam, Kenneth (Hrsg.) (1994), Bilingualism in Deaf Education. Hamburg: Signum, 61-71.

Svirsky, Mario A. (2011), Artificial hearing, natural speech: Cochlear implants, speech production, and the expectations of a high-tech society (review). Language 87: 1, 215-218.

Typolis (o.J.) [Online: http://www.typolis.de/hear/lippenablesen.htm. 10.04.2015].

Volterra, Virginia (1986), What Sign Language Research can teach us about Language Acquisition. Amsterdam: The Institute of German Linguistics of the University of Amsterdam.

WHO (2010), Deafness and hearing loss: [Online: http://www.who.int/ mediacentre/factsheets/fs300/en/index.html. 10.04.2015]. 
Christiane M. Bongartz and Andreas Rohde - 978-3-653-97188-0

Downloaded from PubFactory at 01/11/2019 10:41:16AM

via free access 


\section{Kim Schick \& Andreas Mayer \\ Englischunterricht für Kinder mit Spracherwerbsstörungen}

Children and adolescents with special needs in the area of speech pose a particular challenge for teachers in inclusive settings. Due to their impairment on the phoneticphonological, grammatical, lexico-semantic and pragmatic levels, they experience difficulties processing and reproducing those school matters that are mediated by language (Leonard 2002). In particular, deficits in speech comprehension often lead to problems in the acquisition of curricular contents, as language is the central medium of imparting academic contents in all subjects (Mayer 2009a). English lessons are especially challenging for these students because the English language is both the medium and the learning objective (Rohde \& Lepschy 2007). It is to be expected that the problems of students with speech impairments will be particularly evident in learning a foreign language. Therefore, teachers in inclusive English lessons have to respond in special ways to the needs of those students.

\section{Spezifische Spracherwerbsstörung (SSES)}

Mit Begriffen wie „Sprachbehinderung“, „Sprachbeeinträchtigung“ oder „Förderschwerpunkt Sprache“ werden häufig Auffälligkeiten wie Stottern, Sprechhemmungen oder Probleme mit der Bildung einzelner Laute assoziiert. Aus schulischer Perspektive dagegen ist die „Spezifische Spracherwerbsstörung“ (SSES) aufgrund der Häufigkeit ihres Auftretens und möglicher Auswirkungen auf das schulische Lernen von weit größerer Bedeutung. Mit einer Prävalenz von 6-8\% betroffener Kinder pro Jahrgang handelt es sich bei diesem Störungsbild um eine der häufigsten Entwicklungsbeeinträchtigungen im Kindesalter. An Schulen mit dem Förderschwerpunkt Sprache dürfte sich die Schülerschaft zu etwa $80 \%$ aus Kindern mit Spracherwerbsstörungen rekrutieren.

Bei dieser Problematik handelt es sich um eine komplexe Beeinträchtigung des Spracherwerbs und der Sprachverarbeitung, für die keine offensichtlichen Primärbeeinträchtigungen verantwortlich gemacht werden können (Kannengieser 2014: 188). Symptome der Sprachstörung persistieren häufig bis ins Jugend- und Erwachsenenalter. Bei einem Teil betroffener 
Kinder lassen sich zudem negative Auswirkungen auf andere Entwicklungsbereiche, insbesondere auf den kognitiven, sozialen und emotionalen Bereich nachweisen (Dannenbauer 2001: 100).

„Ja, is hab' eine Haustier, ein Vodel, ja m einmal was habiat, Bubi mag nis in Tüse heibt, dann in Wohnzimmer tommt un mein hesta ansieht. Hilfe ein Vodel in Zimmer, heit und dann hiba Mama afa zu und uns Essen holt von Bubi seine Essen, dann sön essen und hau deht in Täfis, jawoll das' wär's “ (Schöler 1991, zit. nach Mayer 2009b: 125).

Das Beispiel kann verdeutlichen, dass bei spracherwerbsgestörten Kindern unterschiedliche Sprachebenen betroffen sind. So werden bei vielen spracherwerbsgestörten Kindern Symptome auf den Ebenen der Phonologie (Aussprache), der Semantik und des Lexikons (Wortschatz) sowie der Grammatik offensichtlich.

Auf der Ebene der Aussprache haben spracherwerbsgestörte Kinder bisweilen Schwierigkeiten mit der korrekten Produktion einzelner Laute, die ersetzt (z.B. [lole] statt [role], fehlgebildet (z.B. Sigmatismus interdentalis) oder ausgelassen (z.B. [ole] statt [role]) werden. Häufig kommt es zu Vereinfachungsprozessen, die sich bspw. dadurch charakterisieren lassen, dass Laute, die im hinteren Bereich des Mundraums gebildet werden, nach vorne verlagert werden oder Konsonantenfolgen nicht korrekt realisiert werden. Im obigen Beispiel ersetzt das Kind bspw. die Laute [g] und [k] durch [d] und [t] ([fodəl], [tysə]), Konsonantenverbindungen werden durchgängig durch ein [h] ersetzt ([hait] statt [Jrait], [heste] statt $[$ Jveste $])$.

Auf grammatischer Ebene haben spracherwerbsgestörte Kinder Schwierigkeiten, die syntaktischen und morphologischen Regeln ihrer Muttersprache zu erwerben und spontansprachlich anzuwenden. Insbesondere fallen Betroffene durch Probleme mit der Verbzweitstellungsregel im Hauptsatz (z.B. „Ich heute in die Schule gehe.“) und der Verbendstellungsregel in subordinierten Nebensätzen (z.B. „... damit ich lerne lesen und schreiben.“) auf. Auf morphologischer Ebene gehören Schwierigkeiten mit dem Erwerb der Subjekt-Verb-Kontroll-Regel (z.B. „Maxi heute in die Schule gehen.“) und der korrekten Markierung von Akkusativ- und Dativkontexten (z.B. „Der Tiger ist hinter Zaun gerannt.“ „Der Ball liegt hinter der Zaun.“) zu den Kernsymptomen der Störung. 
Auf semantisch-lexikalischer Ebene lassen sich spracherwerbsgestörte Kinder durch eine verzögerte Wortschatzentwicklung charakterisieren. Sie haben Schwierigkeiten, die Bedeutungen von Wörtern und/oder die entsprechenden Wortformen im mentalen Lexikon abzuspeichern, sodass sie im Vergleich zu sprachlich unauffälligen Kindern durch ein deutlich kleineres produktives und rezeptives Lexikon auffallen. Anderen Kindern steht zwar eine mehr oder weniger ausreichende Anzahl an Wörtern im mentalen Lexikon zur Verfügung, jedoch ist die Qualität der Einträge eingeschränkt, sodass sie die Bedeutung der Wörter nur ungefähr kennen bzw. im Vergleich zum eigentlichen Wort eine phonologisch veränderte Form verwenden (z.B. „Tokolomive“ statt „Lokomotive“). Wieder andere Kinder haben - evtl. als Konsequenz der geringen Speicherqualität - Schwierigkeiten mit dem automatisierten Abruf der gespeicherten Einträge („Wortfindungsstörungen“).

Von besonderer Bedeutung ist die Tatsache, dass sich die sprachlichen Schwierigkeiten nicht nur in der produktiven, sondern auch in der rezeptiven Modalität zeigen, dass also auch das Sprachverständnis betroffen ist.

Langfristig besteht die Gefahr, dass sich diese originär spezifisch sprachliche Problematik negativ auf die sozio-emotionale Entwicklung und das schulische Lernen auswirkt. Zahlreiche Forschungsergebnisse aus dem angloamerikanischen Raum machen deutlich, dass bei einem nicht unerheblichen Teil spracherwerbsgestörter Kinder eine geringe Qualität der sozialen Beziehungen nachgewiesen werden kann (Durkin \& Conti-Ramsden 2007: 1449), vermutlich weil betroffene Kinder und Jugendliche Sprache nicht ausreichend gut als Mittel nutzen können, Freundschaften zu knüpfen und aufrecht zu erhalten. Auch was die emotionale Gesundheit angeht, konnte die Forschungsgruppe bei den Parametern Angst und Depression bei spracherwerbsgestörten Kindern im Vergleich zu einer Kontrollgruppe sprachlich unauffälliger Kinder höhere Werte nachweisen (Conti-Ramsden \& Botting 2008: 519f.).

In einer Aufarbeitung des internationalen Forschungsstandes zu den Auswirkungen von Spracherwerbsstörungen auf andere Entwicklungsbereiche kommt Dannenbauer (2002: 14) zu dem Ergebnis, dass bei betroffenen Kindern signifikant häufiger von Aggressivität, Rückzugstendenzen, Konzentrationsstörungen und Ängstlichkeit berichtet wird. Kinder mit Spracherwerbsstörungen erfahren von Altersgenossen weniger Akzeptanz, werden seltener als Spielkameraden ausgewählt, sind seltener in der Lage 
ihr Leben autonom zu gestalten (Conti-Ramsden \& Durkin 2008) und lassen sich durch ein geringeres Selbstwertgefühl charakterisieren (Wadman, Durkin \& Conti-Ramsden 2008). Zudem tragen sie ein deutlich höheres Risiko, Opfer von Mobbing in der Schule zu werden (Knox \& Ramsden 2003: 6), sodass sie zusammenfassend betrachtet Gefahr laufen, „eine AuBenseiterkarriere mit niedrigem Selbstvertrauen und sozialen Problemen zu durchlaufen“ (Grimm 1999, zit. nach Dannenbauer 2001: 106).

Für den vorliegenden Beitrag von besonderer Relevanz sind die negativen Auswirkungen sprachlicher Probleme, insbesondere die erwähnten Schwierigkeiten mit dem Sprachverständnis, auf das schulische Lernen. Trotz aller Bemühungen um Veranschaulichung bleibt die Sprache der Lehrkraft das zentrale Medium des Unterrichts. Lerninhalte, Erklärungen und Arbeitsanweisungen, die die Lehrkraft verbal übermittelt, müssen von Kindern und Jugendlichen zunächst sprachlich verarbeitet werden, bevor sie kognitiv verstanden und in das eigene Wissen integriert werden können. Wenn eine Erklärung zu viele Wörter beinhaltet, die nicht zum Wortschatz eines Kindes gehören, wenn die Erklärung grammatikalische Strukturen beinhaltet, die das Kind nicht verstehen kann, dann fehlen die zwingend notwendigen Voraussetzungen für das Verständnis schulischer Inhalte, auch wenn die kognitiven Fähigkeiten eigentlich vorhanden wären. Erschwerend kommt hinzu, dass von Schulkindern spätestens ab der dritten Klasse erwartet wird, dass sie nun weitgehend selbständig in der Lage sind, Informationen aus Texten zu entnehmen. Hier dürften sich die Schwierigkeiten mit dem Sprachverstehen noch deutlicher zeigen als in der mündlichen Kommunikation. Da in der Schriftsprache zahlreiche nonverbale Informationsträger fehlen, die in der lautsprachlichen Kommunikation zum Entschlüsseln von Botschaften genutzt werden können (räumliche Kontextinformationen, Gestik, Mimik, Prosodie etc.), ist das Leseverständnis sprachlich beeinträchtigter Kinder üblicherweise noch stärker eingeschränkt als das Hörsprachverständnis. Hinzu kommt, dass die grammatikalische Komplexität in Lesetexten üblicherweise anspruchsvoller ist als in der lautsprachlichen Kommunikation und dass auch der Wortschatz abwechslungsreicher ist und Wörter verwendet werden, die in der lautsprachlichen Kommunikation eher selten vorkommen.

Obwohl sich spracherwerbsgestörte Kinder in den ersten Jahrgangsstufen also zusammenfassend dadurch charakterisieren lassen, dass sie mit Schuleintritt das Sprachsystem ihrer Muttersprache noch nicht vollständig erworben 
haben und deshalb in ihrer schulischen Lernentwicklung gefährdet sind, werden sie nun in den meisten Bundesländern bereits ab der ersten Klasse mit dem Erwerb einer ersten Fremdsprache konfrontiert. Da sich Schulen mit dem Förderschwerpunkt Sprache an den Lehrplänen der Regelschulen orientieren, stellt sich diese Herausforderung unabhängig vom Förderort. Grund- und FörderschulpädagogInnen stehen somit vor der anspruchsvollen Aufgabe, Kindern mit Spracherwerbsstörungen spezielle Unterstützungsmaßnahmen anzubieten, die es ihnen ermöglichen, die curricular vorgegebenen Lerninhalte des Fremdsprachenunterrichts zu erwerben. Im Englischunterricht der Primarstufe muss dabei insbesondere auf die semantisch-lexikalische Ebene Rücksicht genommen werden, da der Wortschatzerwerb beim frühen Fremdsprachenerwerb im Zentrum des Unterrichts steht. Aus diesem Grund sollen im Folgenden methodische Anregungen geliefert werden, wie der Englischunterricht für spracherwerbsgestörte Kinder optimiert werden kann.

Dabei können Lehrkräfte, die Englischunterricht für Kinder mit dem Förderschwerpunkt Sprache anbieten, durchaus auf Methoden zurückgreifen, die zum Kernrepertoire eines sprachheilpädagogischen Unterrichts gehören. Auf semantisch-lexikalischer Ebene spielen hier bspw. einige nonverbale Merkmale einer spezifisch akzentuierten Lehrersprache eine wesentliche Rolle. Durch eine spezifische Akzentuierung und Kontrolle ihrer eigenen Sprache kann die Lehrkraft eine effektive Unterstützung bei der Aneignung neuer Wörter anbieten. Indem die Lehrkraft prosodisch möglichst abwechslungsreich spricht, also Veränderungen im Bereich des Sprechtempos, der Betonung, des Rhythmus und der Lautstärke vornimmt, indem sie weiterhin vor einem wichtigen Wort eine kurze Pause einlegt und das potentiell unbekannte Wort dann besonders betont und etwas lauter artikuliert, lenkt sie die Aufmerksamkeit ganz natürlich auf neue Wörter, deren Bedeutung und Aussprache. Notwendig ist es dann, eine sofortige Erklärung zur Bedeutung nachzuliefern (semantische Elaboration) und das neue Wort möglichst hochfrequent zu präsentieren. Um die Kinder zu unterstützen, nicht nur die Bedeutung eines neuen Worts, sondern auch die Wortform im mentalen Lexikon zu verankern, bietet es sich an, insbesondere mehrsilbige komplexe Wörter silbisch gegliedert anzubieten.

Mimik, Gestik und handlungsbegleitende Bewegungen ergänzen und unterstreichen sprachliche Äußerungen und bieten wichtige nonverbale 
Informationsträger, die das Verstehen einer sprachlichen Äußerung effektiv unterstützen können. Auf diese Weise sollte es den Kindern gelingen, eine erste Repräsentation eines Worts im mentalen Lexikon abzuspeichern. Diese Repräsentation in ihrer Qualität zu erhöhen, gelingt dadurch, dass die Kinder motiviert werden, die Wörter selbst hochfrequent zu verwenden. Eine in diesem Zusammenhang wirkungsvolle Hilfe aus der Therapiedidaktik des „Wortschatzsammlers“ von Motsch, Marks \& Ulrich (2015: 119) ist der sogenannte „Zaubertrick“. Bei dieser Strategie werden die Kinder dazu ermutigt, „schwierige“ Wörter gemeinsam mit der Lehrkraft dreimal langsam und mit korrekter Betonung zu sprechen. Anschließend versuchen die Kinder, das Wort ohne Hilfe der Lehrkraft zu benennen, sie überprüfen also ob sie das Wort erfolgreich „in ihren Kopf gezaubert“ haben.

Auf den folgenden Seiten wird eine exemplarische Englischstunde vorgestellt. Es handelt sich um einen Stundenentwurf zum Wortfeld „Clothes“ für den frühen Englischunterricht in der Grundschule. Die Verlaufsplanung bildet den Englischunterricht zunächst so ab, wie er an einer Regelgrundschule durchgeführt werden könnte. Wenngleich der an der Zweitsprachenerwerbsforschung orientierte moderne Englischunterricht - so auch diese Unterrichtsstunde - bereits einige Kriterien des Unterrichts im Förderschwerpunkt Sprache berücksichtigt, könnten weitere sprachheilpädagogische Maßnahmen in die Stunde integriert werden, damit auch spracherwerbsgestörte Kinder möglichst optimale Lernfortschritte erzielen können. Diese Maßnahmen werden in der letzten Spalte des Verlaufsplans „Mögliche Modifikationen aus sprachheilpädagogischer Perspektive“ zunächst lediglich benannt. In Abschnitt 4 dieses Beitrags werden die Maßnahmen ausführlich erläutert. Hier finden sich auch Begründungen, weshalb diese Unterstützungsmaßnahmen für Schüler mit dem Förderschwerpunkt Sprache sinnvoll sind und wie die konkrete methodische Umsetzung aussehen kann. 


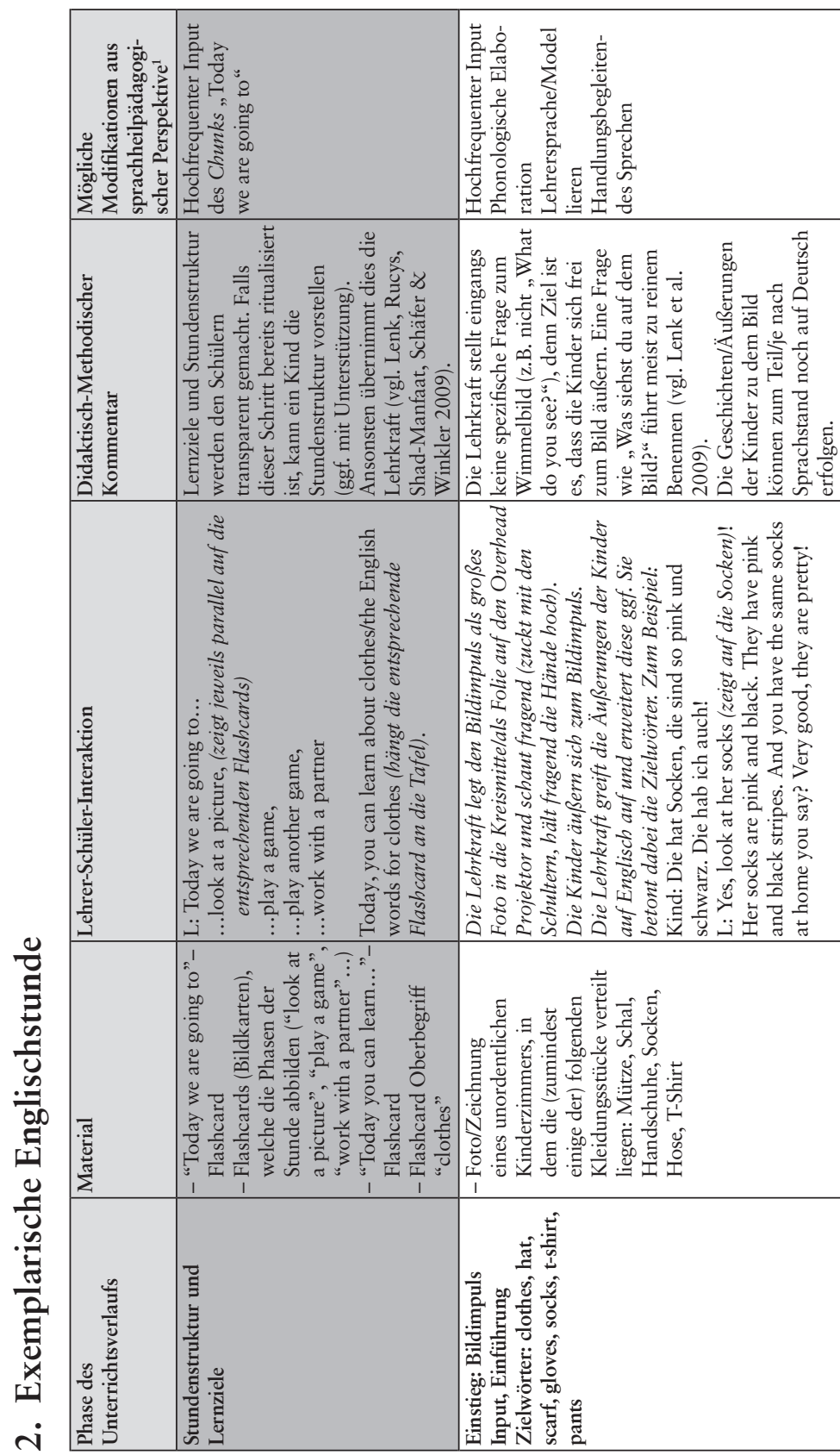

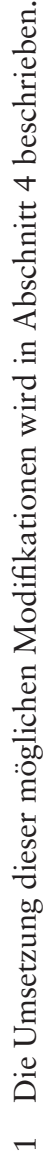

Christiane M. Bongartz and Andreas Rohde - 978-3-653-97188-0 Downloaded from PubFactory at 01/11/2019 10:41:16AM 


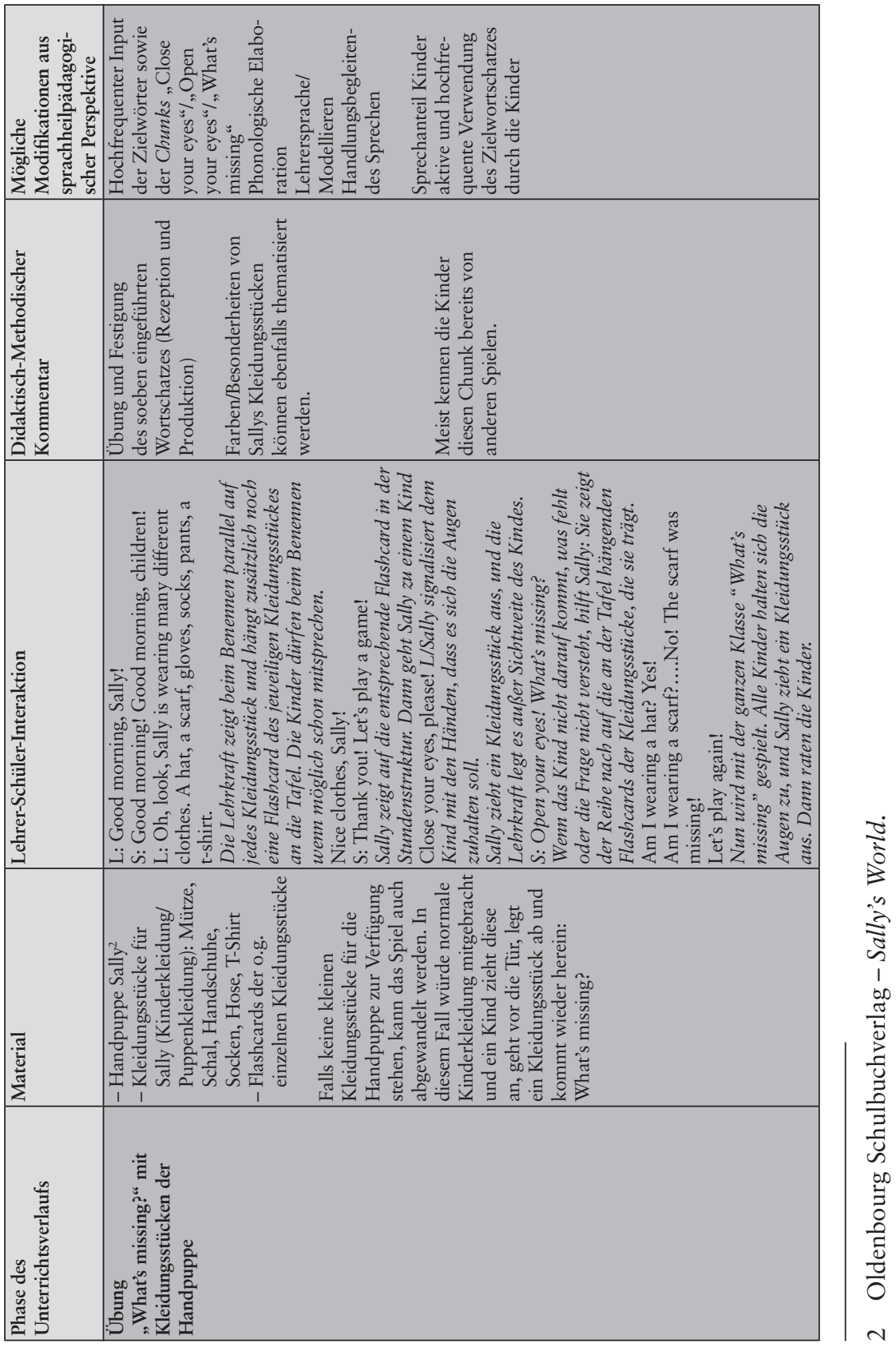

Christiane M. Bongartz and Andreas Rohde - 978-3-653-97188-0 Downloaded from PubFactory at 01/11/2019 10:41:16AM 


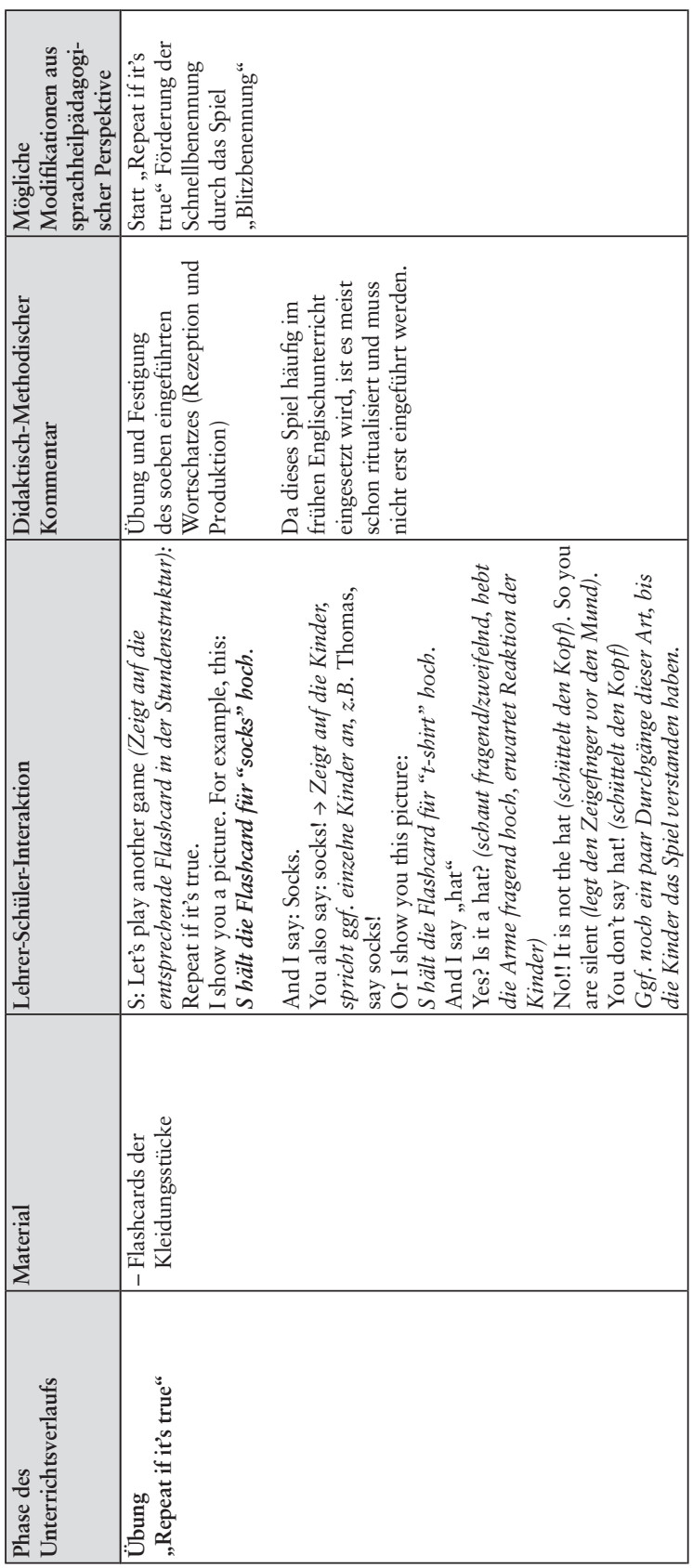

Christiane M. Bongartz and Andreas Rohde - 978-3-653-97188-0 Downloaded from PubFactory at 01/11/2019 10:41:16AM 


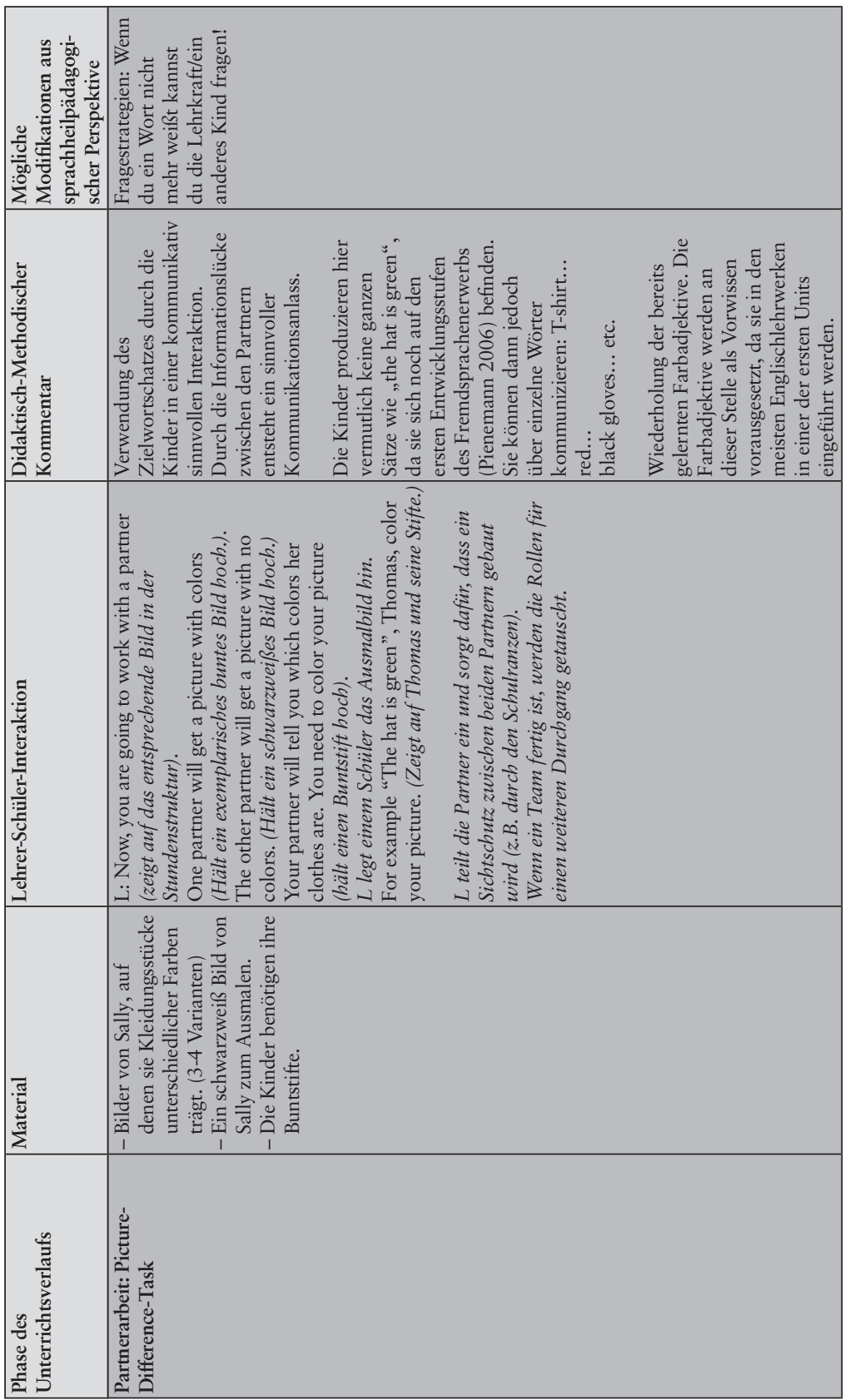

Christiane M. Bongartz and Andreas Rohde - 978-3-653-97188-0 Downloaded from PubFactory at 01/11/2019 10:41:16AM 


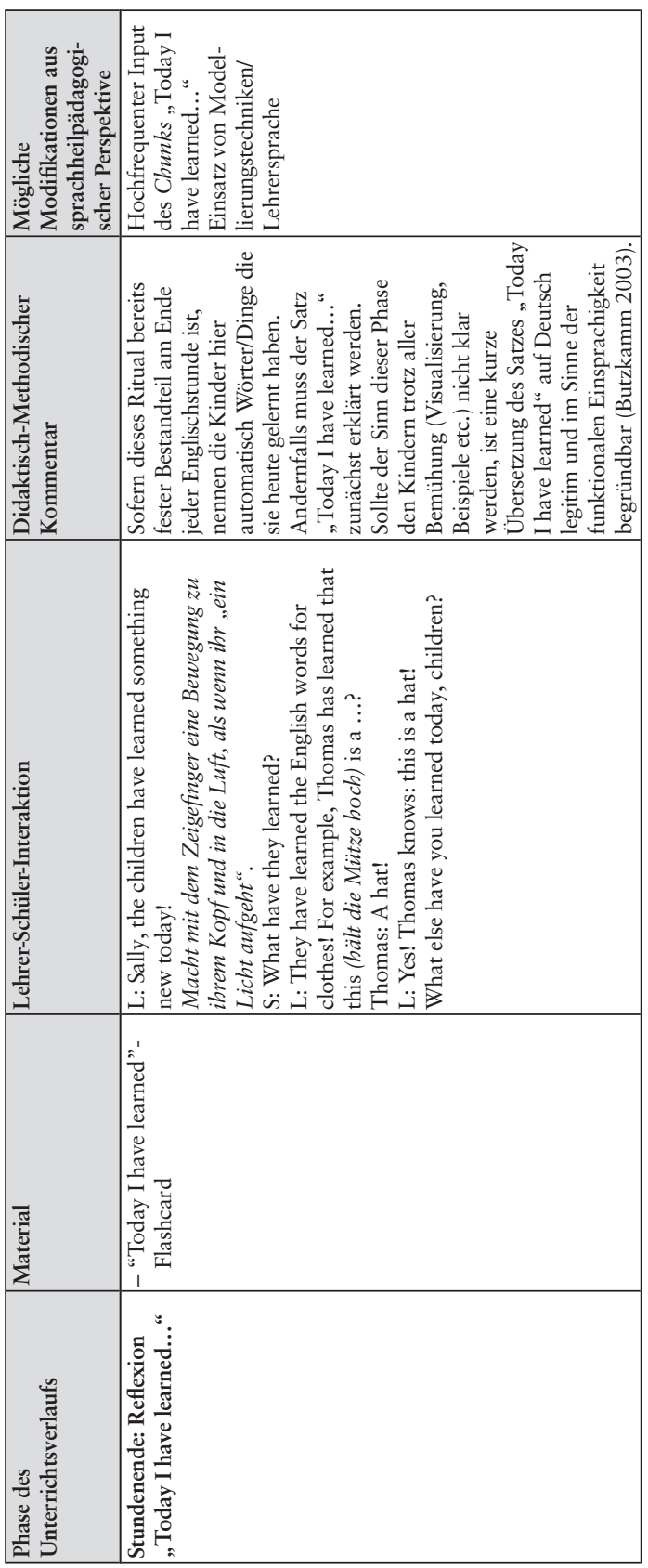

Christiane M. Bongartz and Andreas Rohde - 978-3-653-97188-0

Downloaded from PubFactory at 01/11/2019 10:41:16AM 


\section{Fremdsprachendidaktik meets Sprachheilpädagogik}

Im folgenden Abschnitt werden einige Aspekte der modernen Fremdsprachendidaktik die sich in der oben dargestellten Unterrichtsstunde wiederfinden und mit gängigen Grundprinzipien und Konzepten der schulischen Sprachheilpädagogik übereinstimmen, herausgegriffen und erläutert. Aufgrund einer spracherwerbsorientierten Ausrichtung der Sprachheilpädagogik und der Fremdsprachendidaktik finden sich zahlreiche gemeinsame, ähnliche und sich ergänzende Ansätze und Grundprinzipien. Hier liegt eine große Chance für den inklusiven Unterricht. Lehrkräfte, die sich in ihrer Ausbildung mit Spracherwerb und sprachfördernden Maßnahmen auseinandergesetzt haben, können lernen, dieses sprachfördernde Verhalten bewusster und gezielter für Schüler mit Sprachentwicklungsstörungen einzusetzen.

\subsection{Funktionale Einsprachigkeit}

Im modernen Fremdsprachenunterricht gilt das Prinzip der funktionalen Einsprachigkeit. Die Lehrkraft spricht so viel Englisch wie möglich mit den Kindern. Kurze muttersprachliche Phasen sollten nur in begründeten Ausnahmefällen stattfinden (Kniffka, Rohde, Bongartz, Dahmen \& Siebert-Ott 2008). Übersetzt die Lehrkraft zu häufig auf Deutsch oder bittet einzelne Schüler, Äußerungen für die Klasse zu übersetzen, so entwickeln die Schüler eine Erwartungshaltung: Du übersetzt es ja sowieso gleich. Warum sollte ich mich also bemühen, das Englische zu verstehen? (Kniffka et al. 2008: 10).

In Lehrer-Schüler- oder Schüler-Schüler-Interaktionen auf Englisch können die so wichtigen Bedeutungsverhandlungen (Kapitel 3.2) stattfinden, die den Fremdsprachenerwerb vorantreiben. Die Kinder gewöhnen sich schnell daran, dass die Lehrkraft fast ausschließlich Englisch spricht. Dies funktioniert auch bereits zu Beginn und überfordert die Kinder nicht. Voraussetzung ist, dass die Lehrkraft das Sprachverständnis der Kinder entsprechend unterstützt, indem sie kontextualisiert, d.h. Bilder, Gesten, Mimik, und Rituale nutzt (Kontextualisierung, vgl. Weitz, Pahl, Mattsson, Buyl \& Kalbe 2010: 16ff.). Auch in der in diesem Beitrag dargestellten Unterrichtsstunde (Abschnitt 2, Lehrer-Schüler-Interaktion) wird deutlich, dass dem Prinzip der funktionellen Einsprachigkeit Rechnung getragen wurde. 
Die meisten Englischlehrwerke der Grundschule arbeiten mit einer Leitfigur für die Kinder, die im Lehrwerk immer wieder vorkommt und als Handpuppe in den Unterricht eingebunden werden kann. Die Verwendung von Handpuppen im Fremdsprachenunterricht bringt zahlreiche Vorteile mit sich und ist auch im sprachheilpädagogischen Unterricht fest verankert (Böttger 2005: 93). Im Englischunterricht erfüllt die Handpuppe einige Aufgaben, die mit denen in der Sprachheilpädagogik übereinstimmen. Beispielsweise kann die Handpuppe entlastend wirken, da sie auf freundschaftlicher Augenhöhe mit den Kindern kommuniziert und sich damit von der Lehrkraft unterscheidet (Böttger 2005: 93). Sie darf beispielsweise Fehler machen, stellvertretend nachfragen oder diejenige sein, die etwas selbst am wenigsten weiß oder kann (Böttger 2005: 93).

Darüber hinaus kommen der Handpuppe jedoch noch zusätzliche, für den Unterricht in der Fremdsprache spezifische Aufgaben zu. Sie kann beispielsweise entscheidend dazu beitragen, dass der Englischunterricht überwiegend einsprachig verläuft, da sie nur Englisch spricht und daher die Kommunikation in der Fremdsprache motiviert. Dabei steht sie im Dialog mit der Lehrkraft und den Schülern. Sie kann Arbeitsaufträge, die in der Fremdsprache gegeben werden, verdeutlichen, indem sie vorführt, was die Schüler tun sollen, oder sie kann den Kindern noch unbekannte Inhalte auf kindlicher Ebene erklären bzw. verdeutlichen (Klippel 2000: 31).

\subsection{Bedeutungsverhandlungen (Negotiation of Meaning)}

In einem Englischunterricht, der vorwiegend einsprachig verläuft, entstehen zahlreiche Situationen, in denen die Bedeutungen von Wörtern oder ganzen Äußerungen in der Schüler-Lehrer-Interaktion ausgehandelt werden. Hört ein Kind ein ihm unbekanntes Wort/eine unbekannte Äußerung („Take out your books please!“) so signalisiert das Kind Unverständnis (verwirrter Blick, Nachfragen, der Situation unangemessene Reaktion usw.). Oder aber das Kind hat eine Vermutung, was das Wort bedeutet und äußert diese Vermutung verbal oder durch eine entsprechende Handlung („Heißt book Buch?“/ Das Kind holt sein Mäppchen anstelle des Buches heraus). Der Lerner hat also eine Hypothese über die Bedeutung des Wortes oder der Äußerung gebildet. Der kindliche Sprechanteil in Bedeutungsverhandlungen kann zunächst noch in deutscher Sprache erfolgen (Kniffka et al. 2008: 10). Auf diese kindlichen Handlungen reagiert die Lehrkraft, indem 
sie verständnisstützende Maßnahmen wie Beispiele, handlungsbegleitendes Sprechen, Gestik, Mimik etc. verwendet: „No, not your pencil case“ (zeigt auf das Mäppchen und schüttelt den Kopf), „your book“ (hält das Buch eines Mitschülers hoch), „take out your book!“.

Dialoge dieser Art bezeichnet die Zweitsprachenerwerbsforschung als Bedeutungsverhandlung (eng. Negotiation of Meaning, vgl. Long 1996: 451). Bedeutungsverhandlungen spielen eine bedeutsame Rolle im frühen Fremdsprachenerwerb. Bei einem vorwiegend einsprachig geführten Englischunterricht finden sie auch an der Schule mit dem Förderschwerpunkt Sprache bereits sehr früh statt. So konnten die folgenden Dialoge im Rahmen einer Dissertationsstudie (Schick in Vorb.) im Englischunterricht an einer Schule mit dem Förderschwerpunkt Sprache beobachtet werden:

Beispiel 1: Die Kinder sollen etwas ausmalen.

Lehrkraft: Number two is grey.

Kind: Ich habe kein grey.

Lehrkraft: You can use your pencil.

Kind: Pinsel?

Lehrkraft: Your pencil, look (zeigt ihm den Bleistift)

Kind: Ach Bleistift, der ist ja grey.

\section{Beispiel 2: Ein Kind soll zur Tafel kommen und den Lehrer spielen.}

Lehrkraft: Who wants to be the teacher?

Kind: Was ist teacher?

Lehrkraft: I am a teacher. And Ms. X is a teacher. And Mrs. Y is also a teacher.

Kind: Ah das heißt Frau!

Lehrkraft: No, it's not „woman“. Mr. Z is also a teacher.

Bedeutungsverhandlungen entstehen im Unterricht häufig in spontanen Situationen (ein Schüler kommt zu spät; ein Kind fragt, ob es auf Toilette darf etc.), sofern die Lehrkraft auch hier auf Englisch reagiert (d.h. sich bemüht, ihr Classroom-Management auf Englisch umzusetzen).

Das in der Sprachheilpädagogik bekannte Prinzip der semantischen Elaboration ist der Bedeutungsverhandlung sehr ähnlich. Hier werden die Bedeutungsaspekte bestimmter Zielwörter bewusst verdeutlicht. Das Kind wird mit semantischen Merkmalen des Wortes „gefüttert“. Dies geschieht verbal 
(durch Erklärungen) und/oder durch Beispiele, Handlungen (Ausprobieren), Veranschaulichung (Mitbringen von Realia) etc. D.h. natürliche Mechanismen des Spracherwerbs (Bedeutungsverhandlungen) werden für die sprachheilpädagogische Wortschatzförderung gezielt und bewusst eingesetzt.

„Die Blume hier hat einen Stiel und eine Blüte wie die Tulpe daneben. Das ist eine Rose. Riech mal, wie die Rose duftet. Das Besondere an der Rose sind die Dornen, die sind ganz spitz, an den Dornen der Rose kann man sich ganz fest stechen, da muss man ganz schön vorsichtig sein.“

Das Beispiel zeigt eine semantische Elaboration, wie sie in der Wortschatzförderung der Erstsprache stattfinden kann. Da die Kinder die verbalen Erläuterungen im frühen Englischunterricht nur teilweise verstehen, ist es hier umso bedeutsamer, dass parallel zu den verbalen Erläuterungen Handlungen, Gestik, Mimik, Realia und Bildmaterial zum Einsatz kommen.

\subsection{Sinnvolle Sprechanlässe}

Die Gestaltung des Englischunterrichts orientiert sich u.a. an der interaktionistischen Spracherwerbstheorie. Um eine Fremdsprache erfolgreich erwerben zu können, bedarf es dreier gleich gewichteter Komponenten:

1. verständlichen Sprachinput (Krashen 1985, zit. nach Mitchell et al. 2013: 44f.)

2. die Möglichkeit, in der Fremdsprache in sinnvolle Interaktionen zu treten (Long 1981, 1983a, 1983b, zit. nach Mitchell et al. 2013: 48).

3. die Gelegenheit, Output in der Fremdsprache zu produzieren (Swain 1995, 2005, zit. nach Mitchell et al. 2013: 48).

Auch in sprachtherapeutischen Konzepten wird die Bedeutung aller drei Komponenten (Input-Interaktion-Output) betont (vgl. bspw. Modalitätenwechsel in der Grammatiktherapie, Motsch 2010: 99). Die Berücksichtigung dieser Aspekte sollte also im Englischunterricht für spracherwerbsgestörte Kinder besonders wichtig sein. Alle drei Komponenten finden sich in der in diesem Beitrag vorgestellten Unterrichtsstunde. Bei der Betrachtung des Bildes in der Einstiegsphase kann die Lehrkraft verständlichen englischen Input geben und die Schüler freien Output produzieren. Während der Partnerarbeitsphase erhalten die Schüler die Möglichkeit der Interaktion und der Sprachproduktion. 
Sprechanlässe sollten möglichst sinnvoll gestaltet werden und die Kinder motivieren, die Fremdsprache kommunikativ zu verwenden. Ein sinnvoller Anlass, die Fremdsprache zu verwenden ist immer dann gegeben, wenn in der Partnerarbeit ein Partner andere Informationen hat als der andere, d.h. wenn eine Informationslücke entsteht (Richards \& Rodgers 2014: 186). Dann können diese Informationen nur über einen Austausch miteinander erschlossen werden. Die Einbettung neuer Strukturen in einen kommunikativ sinnvollen Kontext und damit die Verdeutlichung der Funktion von Sprache als Kommunikationsmittel ist auch in der Sprachheilpädagogik ein wichtiges Prinzip (vgl. Glück \& Berg 2010: 105f.; Motsch 2010: 89). Dieses Grundprinzip wird auch in der oben beschriebenen Unterrichtsstunde reflektiert. In der Partnerarbeit haben beide Schüler ein anderes Bild - der eine die farbige Version, der andere das Bild, welches noch ausgemalt werden muss. Nur über einen sprachlichen Austausch miteinander kann die Aufgabe gelöst werden.

\section{Sprachheilpädagogische Modifikation der exemplarischen Englischstunde}

Im Folgenden werden sprachheilpädagogische Modifikationsmöglichkeiten beschrieben, die in der exemplarischen Englischstunde an all den Stellen eingesetzt werden können, an denen in der Stundenübersicht (Kapitel 2) das entsprechende Stichwort in der rechten Spalte vermerkt ist.

\subsection{Hochfrequenter Input}

Im ungestörten Erstsprachenerwerb ist es für Kinder ausreichend, neue Wörter nur wenige Male zu hören, um unmittelbar eine Wortform und ein vorläufiges Konzept zu diesem Wort abzuspeichern. In der Spracherwerbsforschung wird dieses Phänomen als Fast Mapping bezeichnet (Crais 1992). Bei Kindern mit Spracherwerbsstörungen scheinen diese Mapping Prozesse verlangsamt. Diese Kinder müssen neue Wörter der Erstsprache häufiger hören, um sie erfolgreich abzuspeichern (vgl. Leonard 1998: 45f.). Rohde \& Tiefenthal (2000) zu Folge findet das Mapping im Zweit- und Fremdsprachenerwerb sprachnormaler Kinder ebenfalls langsamer statt. Das bedeutet, dass Kinder neue Wörter einer Fremdsprache generell häufiger hören müssen als Wörter der Muttersprache, um sich diese merken zu 
können. Für Kinder mit dem Förderschwerpunkt Sprache dürfte von einer besonderen Notwendigkeit auszugehen sein, neue englische Wörter im Input der Lehrkraft besonders häufig präsentiert zu bekommen. Nur wenn die Lehrkraft die Wörter hochfrequent verwendet, können die Kinder Bedeutung und Wortform abspeichern. Der hochfrequente Input der Lehrkraft kann in der exemplarisch dargestellten Unterrichtsstunde beispielsweise folgendermaßen umgesetzt werden:

Bei der Betrachtung des Bildimpulses aus der exemplarischen Englischstunde (Kapitel 2)

Kind: Die hat Socken, die sind so pink und schwarz. Die hab ich auch!

L: Yes, look at her socks (zeigt auf die Socken)! Her socks are pink and black. The socks have pink and black stripes. And you have the same socks at home you say? Very good, they are pretty socks!

Lehrerzentrierte Unterrichtsphasen, in denen die Lehrkraft ohnehin viel Input gibt oder den Kindern sprachliches Feedback gibt, eignen sich grundsätzlich gut, um Zielwörter/-strukturen hochfrequent und fokussiert anzubieten. Dabei sollte sich die Lehrkraft bewusst machen, welcher Zielwortschatz oder welche Zielstrukturen (Chunks oder bestimmte grammatikalische Strukturen) gerade im Mittelpunkt stehen. Auch Elemente der Lehrersprache sollten hier integriert werden. Eine bewusste Lenkung der Aufmerksamkeit durch prosodisch abwechslungsreiches Sprechen, verbunden mit einer kurzen Pause vor und einer deutlichen Betonung der Zielwörter/-strukturen führen zu einer deutlicheren Wahrnehmung durch die Kinder.

\subsection{Phonologische Elaboration}

Schüler mit SSES benötigen beim Erwerb neuer Wörter nicht nur Unterstützung beim Erlernen der Wortbedeutung, sondern auch bei der Wahrnehmung, Analyse und Abspeicherung der vollständigen Lautstruktur (Phonologie) (vgl. Glück \& Berg 2010: 102f.). Die ungelenkte Begegnung mit neuen Wortformen ist oftmals eine Herausforderung für Kinder mit SSES (vgl. Füssenich 2005; Schlentner 2005). Sie führt auch bzw. insbesondere in der Fremdsprache zu der Speicherung unvollständiger oder diffuser Wortformen. Dies kann sich dann bspw. folgendermaßen äußern: Das Kind speichert... 
- ca...lar statt catterpillar $\rightarrow$ Aitchinson (1997: 175) bezeichnet dies als Badewanneneffekt: Nur Anfangs- und Endsilbe eines Wortes werden gespeichert.

- birk statt bird $\rightarrow$ Ersetzung einzelner Laute

- barit statt rabbit $\rightarrow$ veränderte Lautfolge

- turt statt turtle $\rightarrow$ Auslassung der Endsilbe

Solche unzureichend ausdifferenzierten Repräsentationen der Wortform ermöglichen es dem Lerner allenfalls noch, das korrekte Zielwort rezeptiv wiederzuerkennen. Für eine korrekte Produktion des Zielwortes jedoch ist eine hochwertige mentale Repräsentation der Wortform notwendige Voraussetzung (Glück 1999: 9).

Die Lehrkraft kann die Schüler mit Spracherwerbsstörung aktiv dabei unterstützen, die Phonologie englischer Wörter wahrzunehmen, zu analysieren und möglichst vollständig und korrekt abzuspeichern. Sie kann die Aufmerksamkeit der Schüler verstärkt auf die Wortform lenken, indem sie Wörter in Silben oder subsilbische Einheiten (Onset-Rime oder Einzellaute) segmentiert (Robotersprache) anbietet:

turtle

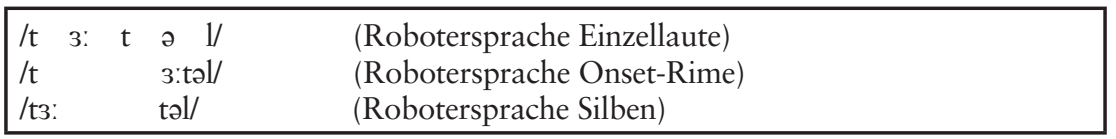

Weiterhin kann sie Wörter langsam und gedehnt aussprechen (Schneckensprache):

/t3: 3: 3: 3: 3: 3: 3:təวəวəlllllllll/

(Schneckensprache)

Bei zunehmender Vertrautheit mit diesen „Sprechweisen“ können auch die Schüler selbst die Wörter auf diese Weise produzieren. Diese Sprechweisen können durch kindgerechte Signalkarten visualisiert werden. 
Abb. 1: Signalkarten zur Verwendung von Schnecken- und Robotersprache, vgl. Jaehner \& Schick 2013: 13, Abb. Copyright Sven Leberer.
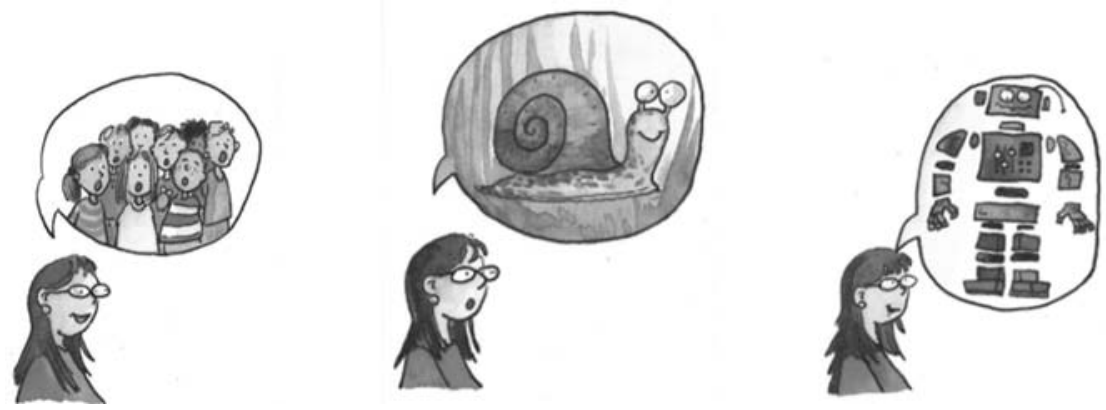

Auf jede Segmentierung oder Dehnung sollte als Kontrast unmittelbar die korrekte Sprechweise des Wortes (also eine Synthetisierung) folgen.

\subsection{Schnellbenennung - automatisierter Wortabruf}

Kinder mit dem Förderschwerpunkt Sprache haben oft Probleme, Wörter in entscheidenden Situationen schnell und mühelos abzurufen. Erhalten Kinder die Gelegenheit, neu gelernte englische Wörter in Übungssituationen möglichst häufig selbst zu produzieren, so kann eine Automatisierung des Wortabrufs erreicht werden (Glück 1999: 24; Nation 2001: 66f., zit. nach Kersten 2010: 72; Vaughn \& Rawson 2011: 1128).

In der Wort-Übungsphase der in diesem Beitrag vorgestellten Unterrichtsstunde kann eine hochfrequente Benennung des neuen Wortschatzes in die Stunde integriert werden. Prinzipiell bietet es sich an, nach Möglichkeit in jeder Englischstunde eine kurze ritualisierte Phase für das Spiel „Blitzbenennung " einzuplanen (beispielsweise am Stundenende).

Noch effektiver zeigt sich dieses Abruftraining, wenn Zielwörter in Übungen möglichst schnell abgerufen werden müssen (Glück 2003: 181; Ulrich 2012: 222). Durch Spiele können Situationen geschaffen werden, in denen es zwingend notwendig und für die Lerner motivierend ist, die Zielwörter häufig und schnell abzurufen. Eine solche Übung ist beispielsweise die Blitzbenennung. Dabei hat die Lehrkraft einen Satz Bildkarten des Zielwortschatzes bei sich, der im Sitzkreis in die Mitte gelegt wird. Die Lehrkraft zeigt in steigender Geschwindigkeit in willkürlicher Reihenfolge auf die Bilder, und die Kinder haben alle gemeinsam die Aufgabe, die Bilder 
so schnell wie möglich zu benennen. Ohne Probleme ist es auch möglich die Blitzbenennung am PC methodisch umzusetzen. Dazu werden die Bilder in eine Power Point-Folie eingefügt und so animiert, dass sie nach dem Start der Präsentation für etwa eine Sekunde aufblitzen und dann wieder verschwinden. Die Frequenz und die Geschwindigkeit können dabei an die Fähigkeiten der Kinder angepasst werden.

\subsection{Fragestrategien}

In der aktuellen Forschung zur Therapie semantisch-lexikalischer Defizite wird zunehmend die Bedeutung von Wortlernstrategien betont. So wurde zuletzt das Therapiekonzept Wortschatzsammler für das Vorschul- (Motsch \& Ulrich 2012) und für das Schulalter (Motsch \& Marks 2015) entwickelt und empirisch evaluiert (vgl. auch Motsch et al. 2015).

Ziel einer Strategietherapie ist es, Kindern mit einem Wortschatzdefizit Strategien zu vermitteln, die es ihnen ermöglichen, ihren Wortschatz auch im Alltag eigenaktiv zu erweitern (Selbstlernmechanismen/Hilfe zur Selbsthilfe). Den Kindern soll es mithilfe der Strategien gelingen, eigenständig „aufzuholen“, d.h. ihren Wortschatzumfang dem von sprachlich normal entwickelten Kindern anzugleichen (Ulrich 2012: 141).

Auch im Englischunterricht kann in Bezug auf den fremdsprachlichen Wortschatzumfang sowie die Speicherqualität einzelner Einträge schnell eine „Lücke“ zwischen Kindern mit SSES und Kindern im normalen Fremdsprachenerwerb entstehen. Aus diesem Grund erscheint es auch hier sinnvoll, schon früh Wortlernstrategien zu vermitteln, welche es Kindern mit SSES ermöglichen, spezifische Mechanismen wie Bedeutungsverhandlungen (Kapitel 3) beim fremdsprachlichen Wortlernen besser/effektiver zu nutzen. Ein Wortlernstrategiekonzept für den frühen Englischunterricht wird aktuell im Rahmen einer Dissertationsstudie (Schick in Vorb.) entwickelt und evaluiert. Ein solches Konzept sollte möglichst an ein Strategietraining in der Erstsprache anknüpfen und dieses sinnvoll für den Fremdsprachenerwerb ergänzen.

Auch im Englischunterricht können Kinder beispielsweise dazu ermutigt werden nachzufragen, wenn sie ein englisches Wort nicht kennen („Was heißt teacher?", „Heißt book Buch?“ „Was heißt Waschbär auf Englisch?“) oder wieder vergessen haben („Was heißt noch mal Schildkröte auf Englisch?“). Neugierde und Frageverhalten haben entscheidenden Einfluss im (Fremd-) 
Sprachenerwerb. Aus diesem Grund sollte Frageverhalten unbedingt positiv verstärkt werden, auch wenn die Kinder insbesondere im frühen Englischunterricht noch häufig auf Deutsch fragen. Schrittweise können wichtige Fragen auch auf Englisch angebahnt werden, wobei die Kinder die Fragen zunächst als Chunks abspeichern werden. Unabhängig davon in welcher Sprache ein Kind gefragt hat, sollte die Reaktion der Lehrkraft möglichst auf Englisch erfolgen. Um sicher zu gehen, dass die Kinder die englische Antwort auf ihre Frage verstehen, können verständnisstützende Maßnahmen und eine angepasste Lehrersprache (Abschnitt 1) zum Einsatz kommen.

In der hier dargestellten Unterrichtsstunde könnten die Kinder beispielsweise vor der Partnerarbeit darauf hingewiesen werden, dass sie andere Kinder nach den englischen Wörtern, die sie für die Aufgabe benötigen, fragen können. Auch während der Einstiegsphase (Bildbetrachtung, freies Erzählen), kann die Lehrkraft die Kinder ermutigen nachzufragen, wenn sie ein Wort nicht (mehr) auf Englisch wissen.

\section{Fazit}

Um spracherwerbsgestörten vergleichbare (Aus-)bildungschancen zu ermöglichen, sollten sie, trotz aller berechtigter Bedenken, die Möglichkeit erhalten, ab der ersten Klasse der Primarstufe eine Fremdsprache zu erlernen. In einer zunehmend globaler werdenden Gesellschaft sollte es für alle Schülerinnen und Schülern eine Selbstverständlichkeit sein, sich auch in einer anderen Sprache angemessen ausdrücken zu können. Dieses Vorhaben wird aber nur gelingen, wenn Kinder mit Schwierigkeiten bei der Aneignung und Umsetzung linguistischen Wissens und sprachlicher Regeln adäquate Unterstützungsangebote erhalten. Der vorliegende Beitrag soll dazu einige methodische Anregungen liefern.

\section{Literaturverzeichnis}

Aitchison, Jean (1997), Wörter im Kopf. Eine Einführung in das mentale Lexikon. Tübingen: M. Niemeyer.

Bäumer, Janina; Bluhm, Janina; Scholz, Rebekka \& Schäfer, Ursula (2009), Early Bird 3/4. Oberursel: Finken Verlag.

Böttger, Heiner (2010), Englisch lernen in der Grundschule (2. Aufl.). Bad Heilbrunn: Julius Klinkhardt. 
Butzkamm, Wolfgang (2003), We only learn language once: The role of the mother tongue in FL classrooms - death of a dogma. Language Learning Journal 28, 29-39.

Conti-Ramsden, Gina \& Durkin, Kevin (2008), Language and independence in adolescents with and without a history of Specific Language Impairment. Journal of Speech, Language and Hearing Research 51, 70-83.

Conti-Ramsden, Gina \& Botting, Nicola (2008), Emotional health in adolescents with and without a history of specific language impairment (SLI). Journal of Child Psychology and Psychiatry 49, 516-525.

Crais, Elizabeth (1992), Fast mapping: a new look at word learning. In: Chapman, R. S. (Hrsg.) (1992), Processes in Language Acquisition and Disorders. St. Louis: Mosby Year Book, 159-185.

Dannenbauer, Friedrich Michael (2001), Prävention aus pädagogischer Sicht (inklusive linguistische und psychologische Perspektiven. In: Grohnfeldt, Manfred (Hrsg.) (2001): Lehrbuch der Sprachbeilpädagogik und Logopädie. Band 3: Diagnostik, Prävention und Evaluation. Stuttgart: Kohlhammer Verlag, 100-111.

Dannenbauer, Friedrich Michael (2002), Spezifische Sprachentwicklungsstörung im Jugendalter. Die Sprachbeilarbeit 47, 10-17.

Durkin, Kevin \& Conti-Ramsden, Gina (2007), Language, social behavior, and the quality of friendships in adolescents with and without a history of specific language impairment. Child Development 78, 1441-1457.

Füssenich, Iris (2005), Frühes Fremdsprachenlernen oder Begegnung mit fremden Sprachen und Kulturen. Die Sprachbeilarbeit 50, 116-122.

Glück, Christian (1999), Wortfindungsstörungen von Kindern in kognitionspsychologischer Perspektive. Der Sprachheilpädagoge 31, 1-27.

Glück, Christian (2003), Semantisch-lexikalische Störungen bei Kindern und Jugendlichen. In: Grohnfeldt, Manfred (Hrsg.) (2003), Lehrbuch der Sprachheilpädagogik und Logopädie. Beratung, Therapie und Rehabilitation. Bd. 4. Stuttgart: Kohlhammer, 178-184.

Glück, Christian \& Berg, Margit (2010), Kugel, Kegel und Zylinder. Wortschatzförderung (nicht nur) im Geometrie-Unterricht: Sprachheilpädagogische Prinzipien und Beispiele. Zeitschrift für Heilpädagogik, 97-108.

Grimm, Hannelore (1999), Störungen der Sprachentwicklung. Göttingen: Hogrefe.

Jaehner, Claudia \& Schick, Kim (2013), Wortschatzarbeit inklusiv. Grundschulmagazin Englisch 1/2013, 10-13. 
Kannengieser, Simone (2014), Spezifische Spracherwerbsstörungen. In: Grohnfeldt, Manfred (Hrsg.) (2014), Grundwissen der Sprachbeilpädagogik und Sprachtherapie. Stuttgart: Kohlhammer Verlag, 188-199.

Kersten, Saskia (2010), The Mental Lexicon and Vocabulary Learning. Implications for the foreign language classroom. Tübingen: Narr Francke Attempto Verlag.

Klippel, Friederike (2000), Englisch in der Grundschule. Berlin: Cornelsen Verlag Scriptor GmbH\&Co. KG.

Kniffka, Gabriele; Rohde, Andreas; Bongartz, Christiane; Dahmen, Silvia \& Siebert-Ott, Gesa (2008), Englisch 1. Lehrerhandreichungen. Berlin u.a.: Duden Paetec GmbH.

Knox, Emma \& Conti-Ramsden, Gina (2003), Bullying risks of 11-year-old children with specific language impairment (SLI): Does school placement matter? International Journal of Language and Communication Disorders 38, 1-12.

Krashen, Stephen (1985), The input hypothesis: issues and implications. Harlow: Longman.

Lenk, Silke; Rucys, Dagmar; Shad-Manfaat, Gerdi; Schäfer, Ursula \& Winkler, Rosemarie (2009), Little Early Bird 1/2. Oberursel: Finken Verlag.

Leonard, Laurence B. (1998), Children with Specific Language Impairment. Cambridge, MA \& London: MIT Press.

Leonard, Laurence B. (2002), Children with Specific Language Impairment. Cambridge, MA \& London: MIT Press.

Long, Michael H. (1981), Input, interaction, and second language acquisition. In: Winitz, Harris (Hrsg.) (1981), Native language and foreign language acquisition (Annals of the New York Academy of Sciences 379), 259-278.

Long, Michael H. (1983a), Native speaker/non-native speaker conversation and the negotiation of comprehensible input. Applied Linguistics 4, 126-141.

Long, Michael H. (1983b), Linguistic and conversational adjustments to non-native speakers. Studies in Second Language Acquisition 5, 177-193.

Long, Michael H. (1996), The role of the linguistic environment in second language acquisition. In: Ritchie, William C. \& Bhatia, Tej K. (Hrsg.) (1996), Handbook of Language Acquisition. San Diego: Academic Press, 413-468. 
Mayer, Andreas (2009a), Dimensionen sprachheilpädagogischen Handelns im Unterricht. Die Sprachheilarbeit 54, 108-118.

Mayer, Andreas (2009b), Ermittlung grammatischer Defizite spracherwerbsgestörter Kinder mittels ESGRAF-R. In: Rosenberger, Katharina (Hrsg.) (2009), Netzwerk Sprache. Kindersprache im Kontext. Wien: Lernen mit Pfiff, 125-152.

Mitchell, Rosamond; Myles, Florence \& Marsden, Emma (2013), Second Language Learning Theories. Oxon: Routledge.

Motsch, Hans-Joachim (2010), Kontextoptimierung. Evidenzbasierte Intervention bei grammatischen Störungen in Therapie und Unterricht (3. Aufl.). München, Ernst Reinhardt.

Motsch, Hans-Joachim \& Ulrich, Tanja (2012), „Wortschatzsammler“ und „Wortschatzfinder“. Effektivität neuer Therapieformate bei lexikalischen Störungen im Vorschulalter. Sprachheilarbeit 57, 70-78.

Motsch, Hans-Joachim; Marks, Dana \& Ulrich, Tanja (2015), Wortschatzsammler. Evidenzbasierte Strategietherapie lexikalischer Störungen im Kindesalter. München: Ernst Reinhardt.

Motsch, Hans-Joachim \& Marks, Dana (2015), Efficacy of the Lexicon Pirate strategy therapy for improving lexical learning in school-age children: A randomized controlled trial. Child Language Teaching and Therapy, 1-19.

Nation, I.S.P. (2001), Learning Vocabulary in Another Language. Cambridge: Cambridge University Press.

Oldenbourg Schulbuchverlag - Sally's World [Online: http://www.oldenbourg.de/osv/1.c.3326787.de. 28.02.2015].

Pienemann, Manfred (2006), Spracherwerb in der Schule. Was in den Köpfen der Kinder vorgeht. In: Pienemann, Manfred; Kessler, Jörg-Uwe; Roos, Eckhard (Hrsg.) (2006), Englischerwerb in der Grundschule. Paderborn: Verlag Ferdinand Schöningh, 33-63.

Richards, Jack C. \& Rodgers, Theodore S. (2001): Approaches and Methods in Language Teaching. Cambridge: Cambridge University Press.

Rohde, Andreas \& Tiefenthal, Christine (2000), Fast mapping in early L2 lexical acquisition. Studia Linguistica 54, 167-174.

Rohde, Andreas \& Lepschy, Almut (2007), „Shoot for the moon“: Englische Immersion in der Grundschule oder Ein Vorschlag, das Dilemma des bilingualen Sachfachunterrichts zu lösen. In: Bosenius, Petra; Donnerstag, 
Jürgen \& Rohde, Andreas (Hrsg.) (2007), Der bilinguale Unterricht Englisch aus der Sicht der Fachdidaktiken. Trier: Wissenschaftlicher Verlag Trier, 1-16.

Schick, Kim (in Vorbereitung), Unterstützende Maßnahmen für den frühen Wortschatzerwerb im Englischunterricht von Schülerinnen und Schüler mit dem Förderschwerpunkt Sprache. Dissertationsprojekt an der Universität zu Köln.

Schlentner, Sabine (2005), Bisherige Erfahrungen mit dem Konzept des „Frühen Fremdsprachenlernens“ an Schulen für Sprachbehinderte in Baden-Württemberg. Die Sprachheilarbeit 50, 140-142.

Schöler, Jutta (1991), Videoaufnahme. Unveröffentliches Transkript. TU Berlin.

Selinker, Larry (1972), Interlanguage. International Review of Applied Linguistics 10, 219-231.

Swain, Merrill (1995), Three functions of output in second language learning. In: Cook, Guy \& Seidlhofer, Barbara (Hrsg.) (1995), Principle and practice in applied linguistics: studies in honour of H.G. Widdowson. Oxford: Oxford University Press, 125-144.

Tiefenthal, Christine (2009), Fast Mapping im natürlichen L2-Erwerb. Trier: Wissenschaftlicher Verlag Trier (WVT).

Ulrich, Tanja (2012), Effektivität lexikalischer Strategietherapie im Vorschulalter. Eine randomisierte und kontrollierte Interventionsstudie. Aachen: Shaker.

Vaughn, Kalif E. \& Rawson, Katherine A. (2011), Diagnosing criterionlevel effects on memory: What aspects of memory are enhanced by repeated retrieval. Psychological Science 22, 1127-1131.

Wadman, Ruth; Durkin, Kevin \& Conti-Ramsden, Gina (2008), Self esteem, shyness and sociability in adolescents with Specific Language Impairment (SLI). Journal of Speech, Language and Hearing Research 51, 938-952.

Weitz, Martina; Pahl, Svenja; Mattsson, Anna; Buyl, Afke \& Kalbe, Elke (2010), The Input Quality Observation Scheme (IQOS): The nature of L2 input and its influence on L2 development in bilingual preschools. In: Kersten, Kristin; Rohde, Andreas; Schelletter, Christina \& Steinlen, Anja K. (Hrsg.) (2010), Bilingual Preschools. Volume 1. Learning and Development. Trier: Wissenschaftlicher Verlag Tier (WVT), 5-44. 
Christiane M. Bongartz and Andreas Rohde - 978-3-653-97188-0

Downloaded from PubFactory at 01/11/2019 10:41:16AM

via free access 


\section{Tatjana Leidig \& Paulina Marnett \\ Selbsteinschätzung im inklusiven Englischunterricht unter besonderer Berücksichtigung des Förderschwerpunktes Emotionale und soziale Entwicklung}

Students with special needs in the areas of emotional and social development, inter alia, often have little confidence in their performance and difficulties in planning, monitoring and checking their work processes. This article presents options of self-evaluation, promoting language learning awareness and metacognition while focusing on primary school inclusive English teaching. The concepts of reflection talks as a routine at the end of a lesson, study tips focusing on learning strategies and self-evaluation forms focusing on learning contents are introduced and their usage, depending on the students' experience is illustrated.

\section{Einleitung}

Der Fachunterricht mit Kindern mit Förderbedarf in der emotionalen und sozialen Entwicklung stellt in der inklusiven Grundschule eine besondere Herausforderung dar. Neben einem geringen Zutrauen in die eigene Leistungsfähigkeit sowie Ängsten vor Misserfolg führen Auffälligkeiten in der Selbstregulation und in der sozialen Interaktion zu ernstzunehmenden Problemen im Unterricht und zu einer geringen Selbstwirksamkeitsüberzeugung (Ellinger \& Wittrock 2005; Jerusalem 2006; KMK 2000; Stein \& Ellinger 2015; Stein 2010). Zudem gehen mangelnde metakognitive Kompetenzen mit Schwierigkeiten im Planen, Überwachen und Kontrollieren der Arbeitsabläufe einher (Myschker \& Stein 2014). Im Englischunterricht stellt sich demzufolge die Aufgabe der Förderung der Selbstreflexionsfähigkeiten sowohl auf fachlicher Ebene im Bereich der „language learning awareness“ als auch auf sonderpädagogischer Ebene.

Der inklusive Englischunterricht fördert über den gezielten Einsatz von Selbsteinschätzungsbögen - in Kombination mit individuellen Feedbackgesprächen - die Fähigkeiten in der realistischen Selbsteinschätzung sowie die Bewusstmachung und das Anerkennen der eigenen Kompetenzen. Konkretes, 
konstruktives Feedback zu Arbeitsprozess und -produkt, sowie Ermutigung stützen die Entwicklung der Selbstwirksamkeit (Berger, Granzer, Looss \& Waack 2013). Mit Selbsteinschätzungsbögen können gerade Kinder mit Beeinträchtigungen in der emotionalen und sozialen Entwicklung über die Verbalisierung des Könnens das Zutrauen in die eigene Leistungsfähigkeit erhöhen.

\section{Theoretische Grundlagen}

Die Förderung von language learning awareness, Selbstregulation und Selbsteinschätzung sind wichtige korrespondierende Ziele des Englischunterrichts in der inklusiven Grundschule.

Die Sprachlernbewusstheit (language learning awareness) steht in engem Zusammenhang zur Sprachbewusstheit (language awareness). Während der Begriff Sprachbewusstheit ${ }^{1}$ das Nachdenken über die Sprache selbst, also über Sprachstrukturen, Gemeinsamkeiten und Unterschiede verschiedener Sprachen, fokussiert, rückt bei der Sprachlernbewusstheit der Lernprozess in den Vordergrund. Demnach impliziert der Begriff ein Nachdenken über das eigene Lernen. Sprachlernbewusstheit ermöglicht die Bewusstmachung eigener Strategien, die Evaluierung deren Effizienz, die Reflexion eigener Lernwege sowie die Fähigkeit, prozedurales Wissen über Lernstrategien und Techniken selbstständig in eigene Lernprozesse einzuschließen (Gnutzmann 2000: 24; Knapp-Potthoff 1997: 13; Kolb 2007: 62).

Die Notwendigkeit einer frühen Anbahnung der Sprachlernbewusstheit ergibt sich u.a. aus bildungspolitischen Forderungen. In den fächerspezifischen Lehrplänen für den Unterricht an Grundschulen des Landes Nordrhein-Westfalen ist für den Bereich Englisch die Fokussierung von Sprachlernbewusstheit im Hinblick auf lebenslanges Lernen explizit formuliert (Richtlinien Grundschule 2008: 71, 75). Zudem wird die Bedeutung gemeinsamer Reflexion über Sprache und Sprachlernkompetenzen gesondert hervorgehoben. Demnach gehört die Ausbildung von Lernstrategien und -techniken zu den Leitzielen des Englischunterrichts. Im Zentrum stehen

1 Das Konzept der Sprachbewusstheit umfasst nach James \& Garrett (1991: 12ff.) kognitive, soziale, politische und affektive Aspekte sowie eine performance domain. Die Autoren ordnen den Begriff der Sprachlernbewusstheit als Teil der performance domain dem Konzept der Sprachbewusstheit zu. 
dabei die Entwicklung autonomen Lernens und der Modellcharakter des frühen Englischlernens zur Übertragung auf weitere Sprachen (Lehrplan Englisch 2008: 72).

Die Sensibilisierung für die Elemente der Sprachlernbewusstheit bildet die Grundlage für die Ausbildung selbstregulierender Kompetenzen. Die Bewusstmachung der Lernwege ermöglicht es dem Lerner, im Sinne des selbstregulierten Lernens (Landmann, Perels, Otto, Schnick-Vollmer \& Schmitz 2015), diese aktiv und planvoll einzusetzen und effektiv aufeinander abzustimmen. Diese Vorgänge erfordern den Rückgriff auf metakognitive Fähigkeiten. ${ }^{2}$ Die Steuerung, Reflexion und Planung des eigenen Lernprozesses gelingt guten Lernern selbstständig, während Kinder mit Defiziten in der Metakognition hier zusätzliche Unterstützung benötigen (Guldimann \& Lauth 2014: 343; Lauth, Grünke \& Brunstein 2014: 263f.). Die metakognitiven Prozesse sind für viele Lerner mit Förderbedarf in der emotionalen und sozialen Entwicklung äußerst herausfordernd; die Regulation des Lernprozesses erfordert aufgrund mangelnder Automatisierung ein derart hohes Maß an Aufmerksamkeit, dass die Kinder mit auftretenden Schwierigkeiten häufig nicht adäquat umgehen können und nicht in der Lage sind, Prozesse adäquat zu steuern, Möglichkeiten abzuwägen und nächste Schritte zu planen. Dies wiederum kann zu unangemessenen Reaktionen in sozialen Situationen, Verweigerung, oppositionellem Verhalten und Motivationsproblemen führen (Menzies, Lane \& Lee 2009). Da der Abruf verschiedener Strategien nicht problemlos gelingt, erschweren vielfach Defizite in der Selbstregulation bei Kindern mit Förderbedarf in der emotionalen und sozialen Entwicklung ein erfolgreiches akademisches Lernen (Bak \& Asaro-Saddler 2013; Ennis, Harris, Lane \& Mason 2014; Mooney, Ryan, Uhing, Reid \& Epstein 2005; Niesyn 2009).

Die bewusste Auseinandersetzung mit Lernprozessen birgt wichtige Implikationen für das lebenslange Fremdsprachenlernen. So ermöglicht die

2 Das Konzept der Metakognition wird unterschiedlich definiert (vgl. Schröder 2000: 642ff.). Hasselhorn \& Labuhn (2008: 29f.) unterscheiden auf der Basis von Hasselhorn (1992) fünf Subkategorien der Metakognition, nämlich systemisches Wissen, epistemisches Wissen, exekutive Prozesse, Sensitivität für die Möglichkeiten kognitiver Aktivitäten und metakognitive Erfahrungen bezüglich der eigenen kognitiven Aktivität. 
Kenntnis über eigene Schwächen und Stärken den weiteren Lernprozess mithilfe von (prozeduralem) Strategiewissen effektiv zu planen und somit selbstorganisiertes Lernen zu fördern. Erfolgreiche Lerner greifen bei neuen und/oder komplexeren Aufgaben immer wieder auf das vorhandene Wissen zurück: Metakognitive Fähigkeiten „bewirken, dass vorhandene Lernstrategien abgerufen und genutzt werden“ (Goldimann \& Lauth 2014: 343), zudem unterstützen sie die Weiterentwicklung derselben. Im Kontext der Sprachlernbewusstheit und des selbstregulierten Lernens erlangen nach Legutke, Müller-Hartmann \& Schocker-v. Ditfurth (2009: 108) vor allem metakognitive Strategien Bedeutung, welche bei der Reflexion des eigenen Lernprozesses Anwendung finden; hierzu zählen Planung, Überwachung und Evaluation des Lernprozesses durch den Lerner (vgl. Böttger 2005: 149; Haudeck 2007: 350; Landmann et al. 2015: 47; Little 1995: 175; Tönshoff 2003: 333).

Um lebenslanges Lernen zu ermöglichen, steht das Wissen über und das Anwenden von Lernstrategien im Zentrum des Fremdsprachenunterrichts. Dabei betont Bransford (2009), dass nicht das Alter des Lerners über die Anwendung von Lernstrategien und den damit erreichbaren Lernzuwachs entscheidet, sondern prozedurales Wissen ${ }^{3}$ über Lernstrategien und deren Einsatzmöglichkeiten (Wolff 2007: 75). Nach Böttger (2005: 149) ist es möglich, Metakognition schon im Grundschulalter entwicklungsgerecht aufzugreifen und gezielt zu fördern. Landmann et al. (2015: 57f.) plädieren für eine frühzeitige Förderung von Lernstrategien und Selbstregulation und betonen die Notwendigkeit einer praktischen Anleitung. Auch für Kinder mit Förderbedarf in der emotionalen und sozialen Entwicklung ist nach Niesyn (2009) eine gezielte Förderung der selbstregulativen Fähigkeiten im Grundschulalter erfolgreich realisierbar; die Effektivität entsprechender Fördermaßnahmen wurde im angloamerikanischen Sprachraum in verschiedenen Studien nachgewiesen. In einem Review zu den Effekten von Interventionen in verschiedenen Bereichen der Selbststeuerung kommen Mooney et al. (2005) zu dem Schluss, dass Schülerinnen und Schüler mit Beeinträchtigungen in der emotionalen und sozialen Entwicklung durch die gezielte Förderung deutliche Fortschritte im akademischen Lernen machen.

3 Im englischsprachigen Raum werden hierfür die Begriffe ,process knowledge sowie ,concept knowledge“ (Bransford 2000: 97) genutzt. 
Erfolg in akademischen Bereichen gilt als eine der Gelingensbedingungen für die erfolgreiche soziale Integration von Kindern mit Förderbedarf in der emotionalen und sozialen Entwicklung im inklusiven Setting (Stein \& Ellinger 2015: 95f.) und erfordert eine intensive, gezielte Förderung der Kinder ausgehend von einer systematischen Analyse der Lernvoraussetzungen. Beeinträchtigungen in der emotionalen und sozialen Entwicklung implizieren häufig geringe Selbstwirksamkeitserwartungen (Furlang, Morrison \& Jimerson 2000: 252), die sich verstetigen und auch auf Motivation und Leistung negativ auswirken können (Schwarzer \& Jerusalem 2002: 39). Da Selbstwirksamkeit einen „Schlüssel zur kompetenten Selbstregulation“ (Schwarzer \& Jerusalem 2002: 37) darstellt, sind vor allem eigene Erfolgserfahrungen von Bedeutung. Kinder mit Förderbedarf in der emotionalen und sozialen Entwicklung benötigen hierzu neben Übungen zur Förderung der Selbstkontrolle und Wissen über Planungs-, Überwachungs- und Regulationsprozesse vor allem „kognitives Wissen über eigene Fähigkeiten, Merkmale von Aufgaben und mögliche Lernstrategien“ (Hartke 2008: 803), das sie dann in Lernprozessen umsetzen können. Dabei hat sich die Vermittlung von Strategien im Bereich Selbstbeobachtung und Selbstkontrolle als besonders wirksam erwiesen (Landrum, Tankersley \& Kauffman 2003).

Zusammenfassend kann festgehalten werden, dass in der neueren Forschung $^{4}$ die Fähigkeit zu metakognitiven Vorgängen als ein lernbarer Prozess verstanden wird, welcher bereits in der Schuleingangsphase angeleitet werden kann. Gleichzeitig betonen verschiedene Autoren, dass vor allem junge Lerner und Kinder mit Beeinträchtigungen in der emotionalen und sozialen Entwicklung einer kontinuierlichen Unterstützung in diesem Prozess bedürfen (Hartke 2008: 803; Legutke et al. 2009: 128; Niesyn 2009: 228; Rolus-Borgward 2002: 101ff.). Hierbei müssen die spezifischen Voraussetzungen von Kindern bezüglich der Rezeptionsfähigkeit sprachlicher Formulierungen und ihre Abstraktionsfähigkeit Beachtung finden. Im inklusiven Englischunterricht können in der Verbindung von Fach- und Entwicklungsebene gezielt Fähigkeiten zur Selbstreflexion aufgebaut werden.

4 Dennoch lassen sich auch in älteren Publikationen Hinweise auf die frühe Ausbildung der Reflexionsfähigkeit finden: „Children begin to reflect on certain properties of language at an early age“" (Clark 1978: 17). 


\section{Ein Beispiel aus der Praxis: Selbsteinschätzungsbögen im Englischunterricht}

Die Sprachlernbewusstheit kann über den bewussten Umgang mit Lernstrategien in Lern- und Reflexionsgesprächen sowie der Selbstevaluation mittels Selbsteinschätzungsbögen gefördert werden. Entsprechend der Altersgruppe und der Anforderungen in inklusiven Kontexten wurde das Konzept konsequent kleinschrittig angelegt. Dabei wird langfristig eine kontinuierliche Progression von der Reflexion und Einschätzung konkreter Unterrichtssituationen hin zu abstrakten Lernzuwächsen angestrebt.

Durch die produktorientierte Selbsteinschätzung mittels Selbsteinschätzungsbögen lernen die Kinder, erworbene Kompetenzen zu reflektieren. Des Weiteren werden in Reflexionsgesprächen prozessorientiert Sprachlernstrategien vorgestellt bzw. bewusst gemacht, Einsatzmöglichkeiten diskutiert und somit der Strategiefundus fortschreitend erweitert, um selbstregulierendes Lernen zu ermöglichen. Weiterhin ermöglichen gemeinsame Unterrichtsgespräche die Reflexion und Auswertung des Unterrichts. Persönliche Lerngespräche bieten direkte, individuelle Rückmeldung zur Selbstevaluation, indem Kriterien für eine realistische Selbsteinschätzung besprochen werden.

Die immanente Betonung erreichter Kompetenzen ermöglicht, die Fähigkeiten zur Selbstregulation und das Erleben der Selbstwirksamkeit der Schülerinnen und Schüler zu stärken. Durch die stetige, handlungsorientierte Auseinandersetzung mit Lernstrategien und Lerntechniken beschäftigen sich die Kinder nicht nur intensiv mit dem eigenen Lernprozess, sondern lernen, selbst Verantwortung für diesen zu übernehmen.

\section{Aufgabentypen der Selbsteinschätzung}

Nach Kolb (2007, 2008) können bei der Konzeption von Selbsteinschätzungsbögen verschiedene Aufgabentypen Berücksichtigung finden. Eine Kombination aus Dokumentation und Selbsteinschätzung bezeichnet Kolb als „Selbsttest“ (Kolb 2007: 200), da die einzuschätzende Kompetenz zunächst ausprobiert und auf Grundlage dieser Probe eingeschätzt wird. Selbsteinschätzungsaufgaben werden in Aufgaben mit und ohne Strategievorgabe unterschieden. Aufgabentypen ohne Strategievorgabe werden wiederum danach differenziert, ob eine direkte Überprüfungsmöglichkeit 
besteht oder nicht. Letzteres tritt immer dann ein, wenn sich die Einschätzung auf eine „interaktive Komponente“ (Kolb 2007: 201) bezieht, wie beispielsweise einen Hörtext. Ist die Möglichkeit einer Überprüfung gegeben, so lässt sich hier weiter im Hinblick auf die Notwendigkeit der Überprüfung differenzieren. So kann eine Einschätzung mittels ikonischer Skalenwerte ohne tatsächliche Überprüfung der Kompetenz vorgenommen werden. Wenn sich die Einschätzung jedoch auf die Dokumentation konkreter Anzahlen bezieht, ist die Überprüfung notwendig.

Die Auswahl der jeweiligen Aufgabentypen im inklusiven Englischunterricht berücksichtigt die Lernausgangslagen der Schülerinnen und Schüler. Im Hinblick auf die Gestaltung der Selbsteinschätzungsbögen ist zu bedenken, dass Kinder mit Förderbedarf in der emotionalen und sozialen Entwicklung vielfach auch Schwierigkeiten im Lesen und Schreiben aufweisen (Bak \& Asaro-Saddler 2013; Ennis et al. 2014), wobei diese wahrscheinlich häufig nicht aus einer Beeinträchtigung der kognitiven Leistungsfähigkeit, sondern aus Problemen in der Aufmerksamkeitssteuerung und Verhaltensproblemen resultieren (Klicpera \& Gasteiger-Klicpera 2008). Adäquate Visualisierung und die Verwendung eindeutiger Symbole stellen eine Orientierungshilfe dar; Aufgabentypen sollten eindeutig und immer gleich gekennzeichnet sein, um die Aufmerksamkeitssteuerung zu unterstützen und Sicherheit durch Wiedererkennen und Ritualisierung zu geben.

\section{Vorarbeit: Reflexionsgespräche am Stundenende}

Um Kompetenzen im Bereich der Selbsteinschätzung anzuleiten und Schritt für Schritt aufzubauen, ist die Etablierung von Reflexionsgesprächen zu Lernstrategien im inklusiven Englischunterricht ein erster wichtiger Schritt. Die nachfolgenden Impulsfragen zur Anleitung von Lern- und Reflexionsgesprächen (Abb. 1 und 2) wurden in Anlehnung an die Vorschläge von Groß (2006), Kolb (2007, 2008) und Sternitzke (2005) erstellt. Freie Lerngespräche fungieren als Einstieg in den bewussten Umgang mit Lernstrategien. Die Impulse sind bewusst offen formuliert und beinhalten affektive Komponenten des Sprachenlernens, um über diese Ebene die Metakognition anzuregen. Die Reflexionsgespräche sollten jeweils zum Stundenende stattfinden und im Sinne eines gelungenen Classroom Managements (Hennemann \& Hillenbrand 2010) als Verfahrensweise ritualisiert in die Unterrichtskultur integriert werden. So können Aspekte der Sprachlernbewusstheit durch 
regelmäßige Wiederholung geübt, gefestigt und in der Folge zunehmend routiniert angewendet werden (Burwitz-Melzer 2008: 184f.).

Die Reflexionsgespräche zielen darauf, verwendete Lernstrategien bewusst zu machen, im Austausch neue Strategien kennen zu lernen und die Effizienz eingesetzter Strategien zu reflektieren, um so wichtige Rückschlüsse auf die Organisation und Planung des eigenen Lernprozesses ziehen zu können. Diese Vorgehensweise stützt für alle Kinder - mit oder ohne Förderbedarf in der emotionalen und sozialen Entwicklung - den Aufbau selbstregulativen Lernens als evidenzbasierte Maßnahme (Mitchell 2014: 105ff.). Die Fragen beziehen sich entweder auf Produkte (Abb. 1) oder Prozesse (Lernstrategien und -techniken) (Abb. 2) des Fremdsprachenlernens. Um den Kindern den Zugang zu erleichtern, kann eine Frage fortlaufend konkretisiert werden.

\section{Abb. 1: Fragen zu Produkten}

Produkt (Wörter, Sätze, Situationen)

o Was hast du heute gelernt?

o Welche Wörter/ Sätze?

o Was kannst du jetzt sagen/ fragen?

o Was kannst du jetzt verstehen?

o konkreter: Kannst du das Gedicht aufsagen?
Abb. 2: Fragen zum Lernprozess

\section{Prozess/ Strategien}

o Wie kannst du dir Wörter/Sätze merken?

o konkreter:

Wie kannst du dir das Wort merken?

o Hast du die Geschichte verstanden a) als ich vorgelesen habe?

b) als $d u$ die Bilder gesehen hast?

Kognitiv anspruchsvoller gestalten sich Fragen bezüglich des Lernprozesses (Abb. 2), da hier über die Feststellung des Lernstandes hinaus prozedurales Wissen formuliert werden muss (Bostelmann 2006: 34ff.). So fördert die Frage „Wie kannst du dir Wörter/Sätze merken?“ die bewusste Auseinandersetzung mit angewendeten Memorierungsstrategien. Auch hier kann eine kontinuierliche Konkretisierung den Einstieg in metakognitive Vorgänge erleichtern. Abschließend erfolgen Reflexionsfragen (Abb. 3), welche die Planung des Lernprozesses in den Blick nehmen. Kinder mit Förderbedarf in der emotionalen und sozialen Entwicklung können hier erfahren, „dass Lernen, Verstehen und Problemlösen keine Alles-oder-Nichts-Prozesse sind (etwas was man kann oder eben nicht kann). Lernen, Verstehen und Problemlösen sind kognitive Vorgänge, die mit Schwierigkeiten behaftet sein 
können, welche es zu überwinden gilt“ (Rolus-Borgward 2002: 106). Das Nachdenken über das eigene Lernen kann nach Guldimann \& Lauth (2014: 350) zudem eine positive Entwicklung des Klassenklimas unterstützen.

Abb. 3: Reflexionsfragen zur Planung des Lernprozesses

\author{
Planung des Lernprozesses \\ o Was findest du noch schwierig? \\ o Was möchtest du noch lernen? Wofür? \\ - konkreter: Möchtest du das noch einmal üben?
}

\title{
Methoden als Ausgangspunkt für die Arbeit mit Selbsteinschätzungsbögen: Lerntipps
}

Ein methodisch orientierter Selbsteinschätzungsbogen bietet sich zur Einführung in zentrale Bereiche der Sprachlernbewusstheit an. Hierzu werden Lerntipps ${ }^{5}$ in Form einer Checkliste für einzelnen Bereiche und Schwerpunkte (Lehrplan Englisch 2008: 73ff.) im Unterricht vorgestellt und diskutiert, um das Bewusstsein für bereits verwendete Lernstrategien zu stärken sowie neue Lernstrategien kennenzulernen, diese zu verbalisieren und sich so auf eine metakognitive Ebene zu begeben. Neben der Anbahnung der Sprachlernbewusstheit ermöglicht die Arbeit mit den Lerntipps ein Herantasten an Formate, Darstellungsarten und schließlich das Ausfüllen eines standardisierten Selbsteinschätzungsbogens.

Das Ausfüllen der Lerntipps erfolgt zunächst im Plenum. Nur so können Unsicherheiten bezüglich formaler und methodischer Schwierigkeiten bei allen Schülern aufgedeckt und beseitigt werden. Die Investition in das Üben der Vorgehensweise zahlt sich hier für Kinder mit und ohne Förderbedarf aus (Niesyn 2009: 230). Die Evaluation übernimmt der Lernende in Einzelarbeit, wobei sich eine dreistufige Skala mittels Emoticons bewährt hat. ${ }^{6}$ Hilfreich ist in diesen Phasen die Arbeit im Lehrkräfteteam. Die Englischlehrkraft kann beispielsweise die Leitung der Gesamtgruppe

5 Als Grundlage für die Erstellung der Lerntipps dienten Vorschläge von Groß (vgl. 2006: 13) und Kolb (vgl. 2007: 141ff.).

6 Zur Selbsteinschätzung stehen den Kindern folgende Zeichen zur Verfügung: smiley $(;)$ (das kann ich schon gut), so-so $:$ (das kann ich noch nicht so gut) und grumpy $:$ (das fällt mir noch schwer). 
übernehmen, während die Lehrkraft für Sonderpädagogik im Sinne der CoTeaching-Form „one teach, one assist“ (Friend, Cook, Hurley-Chamberlain \& Shamberger 2010; Murawski 2012) gezielt Kinder mit Schwierigkeiten in der Selbstregulation unterstützt. Auch eine Aufteilung der Kinder in zwei Lerngruppen im Sinne von „parallel teaching “ könnte eine geeignete Strategie zur Einübung der Selbsteinschätzung sein. Gerade im Zuge der Einführungsphase ist es unerlässlich, mit den Kindern persönliche Feedbackgespräche zu führen, um eine kompetente Selbsteinschätzung anleiten zu können. Kinder mit Beeinträchtigungen in der emotionalen und sozialen Entwicklung benötigen in dieser Phase aufgrund des häufig gering ausgeprägten schulischen Selbstkonzepts eine Lehrkraft, zu der ein vertrauensvolles Verhältnis besteht. ${ }^{7}$

7 Zur Bedeutung der Lehrer-Schüler-Beziehung im Förderschwerpunkt Emotionale und soziale Entwicklung vgl. u.a. Hartke 2008 und Borchert 2008. 


\section{Abb. 4: Selbsteinschätzungsbogen Lerntipps}

Name:

Englisch verstehen

Ich kann Englisch gut verstehen, wenn

- die Lehrerin Bewegungen dazu macht.

- ich Bilder dazu ansehe.
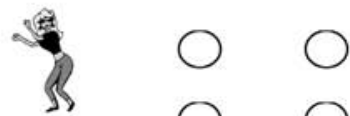

○

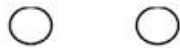

- Jack etwas erzählt.
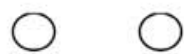

- ich einen Film sehe.
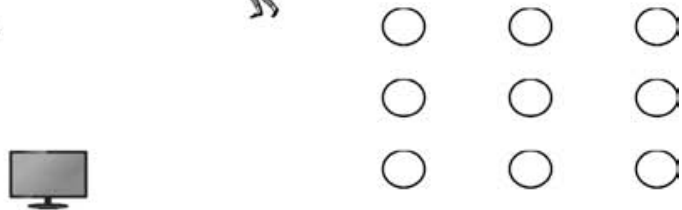

- ich etwas von der CD höre.

Name:

Englisch sprechen

Ich kann englische Wörter und Sätze sprechen, wenn

- ich mit meinem Nachbarn übe.

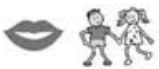

- ich im Chor nachspreche.

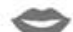

政

- ich Lieder singe.
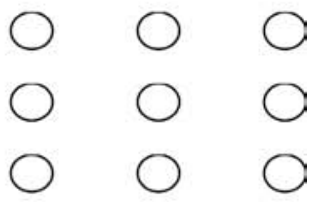

- ich mit Jack spreche.

Name:

Wörter merken

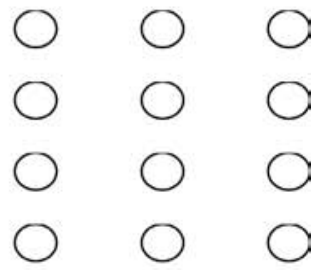

Name:

Ich kann mir englische Wörter merken, wenn

- ich die Wörter oft höre.

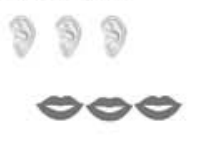

- Wörter in Liedern oder Filmen vorkommen.

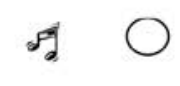

- ich mich dazu bewege.

- ich die Wörter oft spreche.

- Ich dabei an Bilder, Geschichten oder Filme denke.
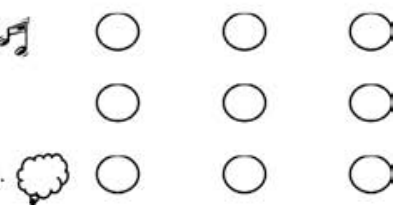


\section{Lerninhalte als Ausgangspunkt für einen Selbsteinschätzungsbogen: Einsatz von Partner- und Kontrollverfahren}

Nach der Einstiegsarbeit mit den Lerntipps konzentriert sich die Selbsteinschätzung zunehmend auf Lerninhalte. ${ }^{8}$ Um die Wiedererkennung zu erleichtern und die Routinebildung zu unterstützen empfiehlt es sich, die Selbsteinschätzungsbögen formal nach gleichem Muster zu konzipieren. Am linken Rand der Aufgabe verdeutlichen vertraute Symbole die jeweils einzuschätzende Fertigkeit. Ein Ohr steht symbolisch für das Hörverstehen, ein Mund analog für die Sprechfertigkeit. Unter der Aufgabe wird die Strategie (z.B. Ankreuzen) verbildlicht. Mit Rücksicht auf die Altersgruppe wird, falls möglich, eine Überprüfungsmöglichkeit ebenfalls durch ein Symbol (z.B. Partnerarbeit) vorgeschlagen. Die Erstbearbeitung erfolgt auch hier im Plenum, um Unsicherheiten und Fragen direkt klären zu können. Im Anschluss an die Selbstevaluation ist eine Sichtung der Selbsteinschätzungsbögen durch die Lehrkraft notwendig. Hierbei können Einschätzungsfehler, welche beispielsweise durch ein gemindertes oder gesteigertes Selbstkonzept auftreten, mittels Fremdevaluation entdeckt werden. Besonders in der Einführungsphase ist es wichtig, Differenzen in der Eigen- und Fremdwahrnehmung (durch die Lehrkraft) aufzudecken und individuell zu besprechen, um die Kinder an eine realistische Selbsteinschätzung heranführen zu können, indem vorhandene Kompetenzen, ggf. im Einzelgespräch, mit Unterstützung abgerufen und somit evaluiert werden können. Hierbei werden den Kindern wiederum Strategien vorgeschlagen, die künftig selbstständig Anwendung finden sollen. In diesen Phasen stellt wiederum die Arbeit im Lehrkräfteteam eine wichtige Gelingensbedingung während der ersten Einführung einer solchen Vorgehensweise dar. In Einzelgesprächen können mit Kindern mit Förderbedarf in der emotionalen und sozialen Entwicklung förderliche Feedbackgespräche im Sinne von Mitchell (2014: 183ff.) erfolgen, die Lernerfolge in den Blick nehmen und bewusst hervorheben.

8 Der systematische Aufbau sowie die formale Gestaltung inhaltlich orientierter Selbsteinschätzungsbögen erfolgte in Anlehnung an die Vorschläge von Groß 2006, Kolb 2007, Legutke 2003 und Rösch 2007. Die Konzeption der Bögen orientierte sich an der Aufgabentypologie von Kolb (2007: 202). 


\section{Abb. 5: Selbsteinschätzung mit Strategievorgabe ohne Überprüfung}

Ich kann diese Früchte auf Englisch sagen:
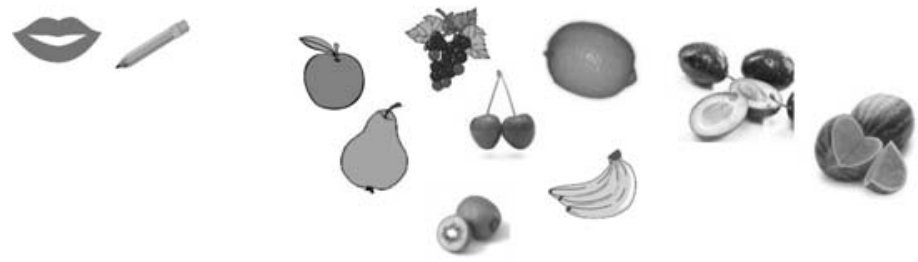

Als Einstieg in die Selbsteinschätzung stellt eine erste Aufgabe (Abb. 5) die „Selbsteinschätzung mit Strategievorgabe ohne Überprüfung“ dar. Durch die Auseinandersetzung mit einzelnen Wortbausteinen wurde die abstrakte Bezeichnung „Ich kann Wörter zum Thema „Fruit“ sagen“ durch die Rückführung auf einzelne Teilbausteine (Wörter) heruntergebrochen. Dies entspricht der Empfehlung von Kolb (2009: 8), über einzelne Wörter im Rahmen konkreter Situationen eine Einschätzung von komplexen Kompetenzen anzuleiten. Die Strategievorgabe besteht hier aus dem Einkreisen der Wörter, wodurch die Aufmerksamkeit des Kindes gezielt auf jedes einzelne Wort (Bild) gelenkt wird. Die Strategievorgabe „Einkreisen“ bietet sich für sehr junge Lerner an, da das Schriftbild für eine mögliche Dokumentation noch nicht ausreichend gefestigt ist und zudem die Überprüfung einer weiteren Fertigkeit (Schreiben) impliziert wäre. Da hier zu einer realistischen Selbsteinschätzung keine Überprüfung in Einzelarbeit oder mit einem Partner vorgesehen ist - das Kind könnte die Früchte willkürlich einkreisen bzw. nicht einkreisen -, werden Überprüfungsmöglichkeiten gemeinsam besprochen.

Im folgenden Beispiel soll eingeschätzt werden, ob Aussagen zur Farbe, zu Vorlieben oder Abneigungen gegenüber Früchten getroffen werden können (Abb. 6). Hier wurde als Aufgabenformat eine Selbsteinschätzung mit Strategievorgabe (Vorsprechen) und Überprüfungsmöglichkeit (Partner) gewählt. Das Kind soll sich so in die konkrete Sprachhandlungssituation begeben und die Kompetenz erproben. Der Partner dient dabei als zusätzliche Überprüfungsinstanz (Kolb 2007: 209). Dies erscheint insofern sinnvoll, da im Anbahnungsprozess häufig das Bedürfnis nach einer Fremdeinschätzung geäußert wird (Legutke 2003: 5). Die Arbeit mit einem Partner kann für Kinder mit Beeinträchtigungen in der emotionalen und sozialen Entwicklung eine wichtige Unterstützung sein; Maßnahmen des peer-tutorings gelten in 
sonderpädagogischen und inklusiven Settings als wirksam (Mastropieri \& Scruggs 2010; Mitchell 2014: 47ff.). Bei der Etablierung von Lernpartnerschaften (Guldimann \& Lauth 2014: 346) ist von Seiten der Lehrkräfte auf Stabilität, Passung der Paare, Anleitung und konkrete Anweisungen für die Zusammenarbeit zu achten.

\section{Abb. 6: Selbsteinschätzung mit Strategievorgabe und Überprüfungsmöglichkeit Partner}

\section{Ich kann sagen,}

- welche Farbe eine Frucht hat, $\bigcirc$

- ob ich eine Frucht mag,

- oder nicht mag
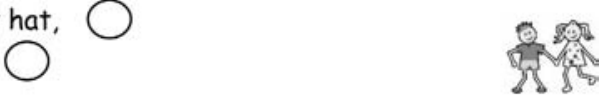

\section{Selbsteinschätzungen für das Formulieren von Zielen für das eigene Lernen nutzen}

Die vorgestellten Selbsteinschätzungen können im inklusiven Englischunterricht als Ausgangspunkt für das Entwickeln von Zielen auf der Ebene der Lernstrategien genutzt werden. Das Setzen von Nahzielen unterstützt den Aufbau einer positiven Selbstwirksamkeitserwartung (Schwarzer \& Jerusalem 2002: 45f.). Aus sonderpädagogischer Sicht ist es für Kinder mit Beeinträchtigungen in der emotionalen und sozialen Entwicklung besonders wichtig, dass die - im ersten Schritt im Einzelgespräch - erarbeiteten Ziele realistisch, kleinschrittig, beobachtbar und zeitnah zu erreichen sind. Die jeweils avisierten Ziele sollten besprochen, inhaltlich klar gefüllt, intensiv geübt, ggf. auch demonstriert und in regelmäßigen Abständen reflektiert werden (Farley, Torres, Wailehua \& Cook 2012: 40; Menzies et al. 2009: 28; Mitchell 2014: 254f.). Der inklusive Englischunterricht kann so durch die Förderung der language learning awareness und der Metakognition einen Beitrag auf fachlicher und überfachlicher Ebene leisten, um das Erleben von schulischen Erfolgen zu unterstützen und damit das Selbstwirksamkeitserleben aller Kinder zu fördern. 


\section{Literaturverzeichnis}

Bak, Nicole \& Asaro-Saddler, Kristie (2013), Self-Regulated Strategy Development for Students With Emotional Behavioral Disorders. Beyond Behavior 22: 3, 46-53.

Berger, Regine; Granzer, Dietlinde; Looss, Wolfgang \& Waack, Sebastian (2013), „, Warum fragt ihr nicht einfach uns?" Mit Schüler-Feedback lernwirksam unterrichten. Weinheim \& Basel: Beltz.

Borchert, Johann (2008), Lehrer-Schüler-Interaktionen. In: GasteigerKlicpera, Barbara; Julius, Henri \& Klicpera, Christian (Hrsg.) (2008), Sonderpädagogik der sozialen und emotionalen Entwicklung. Göttingen: Hogrefe, 740-751.

Bostelmann, Antje (Hrsg.) (2006), Das Portfolio-Konzept in der Grundschule. Individualisiertes Lernen organisieren. Mühlheim: Verlag an der Ruhr.

Böttger, Heiner (2005), Englisch lernen in der Grundschule. Bad Heilbrunn: Klinkhardt.

Bransford, John D.; Brown, Ann L. \& Cocking, Rodney R. (2000), How People Learn. Brain, Mind, Experience and School. Washington, D.C.: National Academy Press.

Burwitz-Melzer, Eva (2008), Ein neues Portfolio für den Fremdsprachenunterricht - Übergang und Selbstevaluation. In: Grau, Maike \& Legutke, Michael K. (Hrsg.) (2008), Fremdsprachen in der Grundschule. Auf dem Weg zu einer neuen Lern- und Leistungskultur. Beiträge zur Reform der Grundschule 126. Frankfurt/Main: Grundschulverband, Arbeitskreis Grundschule, 170-193.

Clark, Eve V. (1978), Awareness of language: Some evidence from what children say and do. In: Sinclair, Anne; Jarvella, Robert J. \& Levelt, Willem J.M. (Hrsg.) (1978), The child's conception of language. Berlin, Heidelberg: Springer, 17-43.

Ellinger, Stephan \& Wittrock, Manfred (Hrsg.) (2005), Sonderpädagogik in der Regelschule: Konzepte - Forschung - Praxis. Stuttgart: Kohlhammer.

Ennis, Robin Parks; Harris, Karen R.; Lane, Kathleen Lynne \& Mason, Linda H. (2014), Lessons Learned from Implementing Self-Regulated Strategy Development with Students with Emotional and Behavioral Disorders in Alternative Educational Settings. Behavioral Disorders 40: 1, 68-77. 
Farley, Cynthia; Torres, Caroline; Wailehua, Cat-Uyen T. \& Cook, Lysandra (2012), Evidence-Based Practices for Students With Emotional and Behavioral Disorders: Improving Academic Achievement. Beyond Behavior 21: 2, 37-43.

Friend, Marilyn; Cook, Lynne; Hurley-Chamberlain, Deanna \& Shamberger, Cynthia (2010), Co-Teaching: An Illustration of the Complexity of Collaboration in Special Education. Journal of Educational and Psychological Consultation 20: 1, 9-27.

Furlong, Michael J.; Morrison, Gale M. \& Jimerson, Shane R. (2004), Externalizing Behaviors of Aggression and Violence and the School Context. In: Rutherford, Robert Bruce; Quinn, Mary M. \& Mathur, Sarup R. (Hrsg.) (2004), Handbook of Research in Emotional and Behavioral Disorders. New York: Guilford Press, 243-261.

Gasteiger, Christian \& Gasteiger-Klicpera, Barbara (2008), Lernstörungen und Störungen der sozialen und emotionalen Entwicklung. In: GasteigerKlicpera, Barbara; Julius, Henri \& Klicpera, Christian (Hrsg.) (2008), Sonderpädagogik der sozialen und emotionalen Entwicklung. Göttingen: Hogrefe, 353-364.

Gnutzmann, Claus (2000), Grammatik lehren und lernen. Zu den allgemeinen Lernzielen des schulischen Grammatikunterrichts. In: Düwell, Henning; Gnutzmann, Claus \& Königs, Frank. G. (Hrsg.) (2000), Dimensionen der didaktischen Grammatik. Festschrift für Günther Zimmermann zum 65. Geburtstag. Bochum: AKS-Verlag, 67-82.

Groß, Christiane (2006), Die Arbeit mit einem Sprachenportfolio in der Grundschule. Ein Unterstützungsangebot für Lehrkräfte. BLK-Verbundprojekt Sprachen lehren und lernen als Kontinuum. [Online: http://www. sprachenportfolio.de/Modul3/pdf/NW/Gross1.pdf 17.03.2015].

Guldimann, Titus \& Lauth, Gerhard W. (2014), Förderung von Metakognition und strategischem Lernen. In: Lauth, Gerhard W.; Grünke, Matthias \& Brunstein, Joachim C. (Hrsg.) (2014), Interventionen bei Lernstörungen. Förderung, Training und Therapie in der Praxis (2. Aufl.). Göttingen: Hogrefe, 341-352.

Hartke, Bodo (2008), Spezifische Unterrichtsprinzipien. In: GasteigerKlicpera, Barbara; Julius, Henri \& Klicpera, Christian (Hrsg.) (2008), Sonderpädagogik der sozialen und emotionalen Entwicklung. Göttingen: Hogrefe, 797-810. 
Hasselhorn, Markus (1992), Metakognition und Lernen. In: Nold, Günter (Hrsg.) (1992), Lernbedingungen und Lernstrategien. Welche Rolle spielen kognitive Verstehensstrukturen? Tübingen: Narr, 35-63.

Hasselhorn, Marcus \& Labuhn, Andju S. (2008), Metakognition und selbstreguliertes Lernen. In: Schneider, Wolfgang \& Hasselhorn Marcus (Hrsg.) (2008), Handbuch Pädagogische Psychologie. Göttingen: Hogrefe, 28-37.

Haunss, Jeanette; Kaden, Elke \& Werlen, Erika (2015), Ich kann's! Das Sprachenportfolio in der Grundschule. Grundschulmagazin Englisch 1/2005, 6-8.

Hennemann, Thomas \& Hillenbrand, Clemens (2010), Klassenführung Classroom Management. In: Hartke, Bodo; Koch, Katja \& Diehl, Kerstin (Hrsg.) (2010), Förderung in der schulischen Eingangsstufe. Stuttgart: Kohlhammer, 255-279.

James, Carl \& Garrett, Peter (1991) (Hrsg.), Language Awareness in the Classroom. Burnt Mill, Harlow, Essex: Longman.

Jerusalem, Matthias (2006), Motivationale und volitionale Voraussetzungen des Unterrichts. In: Arnold, Karl-Heinz; Sandfuchs, Uwe \& Wiechmann, Jürgen (Hrsg.) (2006), Handbuch Unterricht. Bad Heilbrunn: Klinkhardt, 575-579.

Knapp-Potthoff, Annelie (1997), Sprach(lern)bewußtheit im Kontext. Fremdsprachen Lehren und Lernen 26, 9-23.

Kolb, Annika (2007), Portfolioarbeit, Wie Grundschulkinder ibr Sprachenlernen reflektieren. Tübingen: Narr, Giessener Beiträge zur Fremdsprachendidaktik.

Kolb, Annika (2008), Da können wir wissen, was wir wissen. Wie Kinder ihr sprachliches Können einschätzen. In: Grau, Maike \& Legutke, Michael K. (Hrsg.) (2008), Fremdsprachen in der Grundschule. Auf dem Weg zu einer neuen Lern- und Leistungskultur. Frankfurt/Main: Grundschulverband, Arbeitskreis Grundschule, Reihe: Beiträge zur Reform der Grundschule 126, 194-210.

Kolb, Annika (2009), Portfolioarbeit in der Praxis - Was kann ich schon? Grundschulmagazin Englisch 4/2009, 6-8.

Kuhn, Tatjana (2006), Grammatik im Englischunterricht der Primarstufe. Theoretische Grundlagen und praktische Unterrichtsvorschläge. Heidelberg: Winter. 
Kultusministerkonferenz (KMK) (2000), Empfehlungen zum Förderschwerpunkt emotionale und soziale Entwicklung. [Online: http://www.kmk.org/ fileadmin/pdf/PresseUndAktuelles/2000/emotsozentw.pdf. 21.03.2015].

Landmann, Meike; Perels, Franziska; Otto, Barbara; Schnick-Vollmer, Kathleen \& Schmitz, Bernhard (2015), Selbstregulation und selbstreguliertes Lernen. In: Wild, Elke \& Möller, Jens (Hrsg.) (2015), Pädagogische Psychologie (2. Aufl.). Heidelberg: Springer, 45-65.

Landrum, Timothy J.; Tankersley, Melody M. \& Kauffmann, James M. (2003), What is Special About Special Education for Students with Emotional or Behavioral Disorders? The Journal of Special Education 37: 3, 148-156.

Lauth, Gerhard W.; Grünke, Matthias \& Brunstein, Joachim C. (2014), Vermittlung von Lernstrategien und selbstreguliertem Lernen. In: Lauth, Gerhard W.; Grünke, Matthias \& Brunstein, Joachim C. (Hrsg.) (2014), Interventionen bei Lernstörungen. Förderung, Training und Therapie in der Praxis (2. Aufl.). Göttingen: Hogrefe, 262-276.

Legutke, Michael K. (2003), Portfolio der Sprachen. Eine erfolgversprechende Form der Lernstandsermittlung? Primary English 1/2003, 4-6.

Legutke, Michael K.; Müller-Hartmann, Andreas \& Schocker-v.Ditfurth, Marita (2009), Teaching English in the Primary School. Stuttgart: Klett.

Little, David (1995), Learning as dialogue: the dependence of learner autonomy on teacher autonomy. System 23: 2, 175-181.

Mastropieri, Margo \& Scruggs, Thomas E. (2000), The inclusive classroom: Strategies for effective instruction. Upper Saddle River, New Jersey: Prentice-Hall.

Menzies, Holly M.; Lane, Kathleen Lynne \& Lee, Johanna Marie (2009), Self-Monitoring Strategies for Use in the Classroom: A Promising Practice to Support Productive Behavior for Students With Emotional or Behavioral Disorders. Beyond Behavior 18: 2, 27-35.

Ministerium für Schule und Weiterbildung des Landes Nordrhein-Westfalen (Hrsg.) (2008), Richtlinien und Lehrpläne für die Grundschule in Nordrhein-Westfalen. Frechen: Ritterbach.

Mitchell, David (2014), What Really Works in Special and Inclusive Education. Using evidence-based teaching strategies (2. Aufl.). London \& New York: Routledge. 
Mooney, Paul; Ryan, Joseph B.; Uhing, Brad M.; Reid, Robert \& Epstein, Michael H. (2005), A Review of Self-Management Interventions Targeting Academic Outcomes for Students with Emotional and Behavioral Disorders. Journal of Behavioral Ecucation 14: 3, 203-221.

Murawski, Wendy (2012), 10 Tips for Using Co-Planning Time More Efficiently. Teaching Exceptional Children 44: 4, 8-15.

Myschker, Norbert \& Stein, Roland (2014), Verhaltensstörungen bei Kindern und Jugendlichen (7. Aufl.). Stuttgart: Kohlhammer.

O’Malley, J. Michael; Chamot, Anna Uhl \& Anderson, Neil J. (1990), Learning strategies in second language acquisition. Cambridge: Cambridge University Press.

Rolus-Borgward, Sandra (2002), Der Einfluss metakognitiver und motivationaler Faktoren auf die schulische Leistung von Kindern und Jugendlichen mit Lern- und Verhaltensstörungen. In: Schröder, Ulrich \& Wittrock, Manfred (Hrsg.) (2002), Lernbeeinträchtigung und Verhaltensstörung. Konvergenzen in Theorie und Praxis. Stuttgart: Kohlhammer, 96-107.

Rubin, Joan; Chamot, Anna Uhl; Harris, Vee \& Anderson, Neil J. (2007), Intervening in the use of strategies. In: Cohen, Andrew D. \& Macaro, Ernesto (Hrsg.) (2007), Language Learner Strategies. Oxford: Oxford University Press, 141-185.

Schwarzer, Ralf \& Jerusalem, Matthias (2002), Das Konzept der Selbstwirksamkeit. In: Jerusalem, Matthias \& Hopf, Diether (Hrsg.) (2002), Selbstwirksamkeit und Motivationsprozesse in Bildungsinstitutionen (Zeitschrift für Pädagogik, Beiheft, 44). Weinheim: Beltz, 28-53.

Schröder, Ulrich (2000), Metakognition. In: Borchert, Johann (Hrsg.) (2000), Handbuch der Sonderpädagogischen Psychologie. Göttingen: Hogrefe, 642-653.

Stein, Roland \& Ellinger, Stephan (2015), Zwischen Separation und Inklusion: zum Forschungsstand im Förderschwerpunkt emotionale und soziale Entwicklung. In: Stein, Roland \& Müller, Thomas (Hrsg.) (2015), Inklusion im Förderschwerpunkt emotionale und soziale Entwicklung. Stuttgart: Kohlhammer, 76-109.

Stein, Roland (2010), Unterricht. In: Ahrbeck, Bernd \& Willmann, Marc (Hrsg.) (2010), Pädagogik bei Verhaltensstörungen - ein Handbuch. Stuttgart: Kohlhammer, 258-268. 
Sternitzke, Ruth (2005), How did I do? Reflexionsgespräche als Vorbereitung für die Selbstbewertung. Grundschulmagazin Englisch 1/2005, 11-13.

Weskamp, Ralf (2001), Fachdidaktik. Grundlagen \& Konzepte. Anglistik/ Amerikanistik. Berlin: Cornelsen.

Wolff, Dieter (2007), Lernstrategien beim Fremdsprachenlernen. In: Timm, Johannes-Peter (Hrsg.) (2007), Englisch lernen und lehren. Didaktik des Englischunterrichts. Berlin: Cornelsen, 70-77. 